\title{
Mobilität auf dem Arbeitsmarkt für Ältere die Rolle der Betriebe
}

\author{
Von der Fakultät Wirtschaft \\ der Leuphana Universität Lüneburg
}

zur Erlangung des Grades

Doktor der Wirtschafts- und Sozialwissenschaften (Dr. rer. pol.)

genehmigte

Dissertation

von

Derik Burgert

aus

Kapstadt / Südafrika 
Eingereicht am: 09.09.2009

Mündliche Prüfung am: 11.12.2009

Erstgutachter: Prof. Dr. Merz

Zweitgutachter: Prof. Dr. Wagner

Prüfungsausschuss: Prof. Dr. Merz, Vors.

Prof. Dr. Wagner

Prof. Dr. Martin

Elektronische Veröffentlichung des Dissertationsvorhabens inkl. einer Zusammenfassung unter dem Titel:

Mobilität auf dem Arbeitsmarkt für Ältere - die Rolle der Betriebe Veröffentlichungsjahr: 2011

Veröffentlicht im Onlineangebot der Universitätsbibliothek unter der URL:

http://www.leuphana.de/ub 


\section{Inhalt}

TABELLENVERZEICHNIS

ABBILDUNGSVERZEICHNIS...................................................................... VI

ABKÜRZUNGSVERZEICHNIS ....................................................................... VII

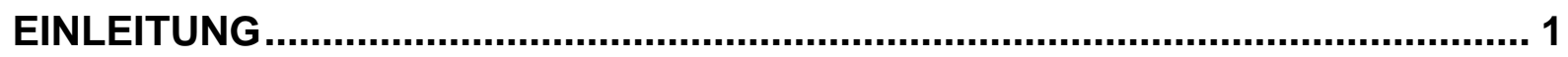

\section{A AUSTRITT AUS DEM ERWERBSLEBEN: EINE MIKROANALYSE DER} BETRIEBLICHEN EINFLÜSSE ............................................................... 8

A 1 Theoretische Grundlagen: Erwerbsaustritt in der Diskussion .....................................................9 9

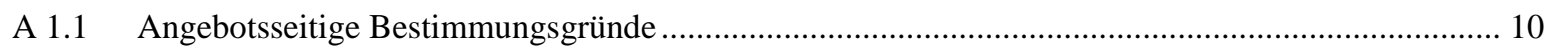

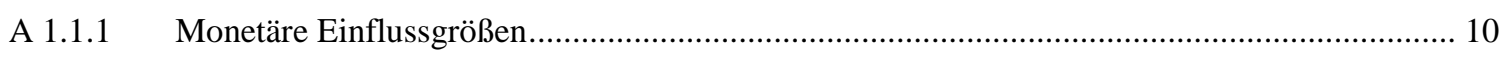

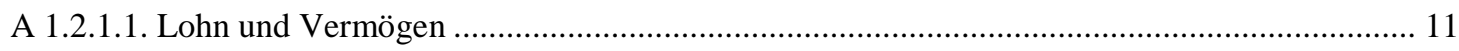

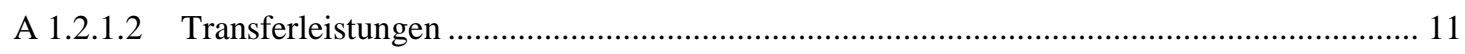

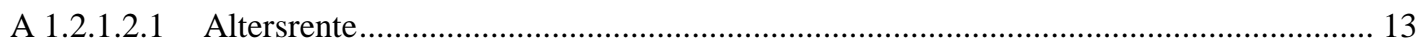

A 1.2.1.2.2 Berufs- und Erwerbsunfähigkeit ......................................................................... 19

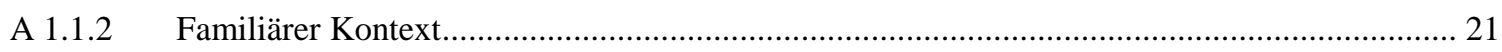

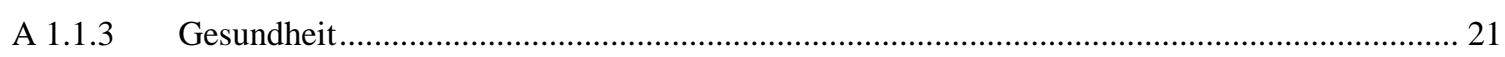

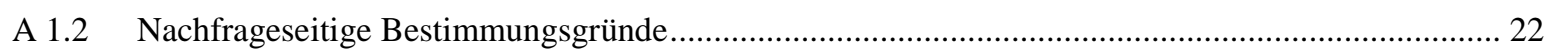

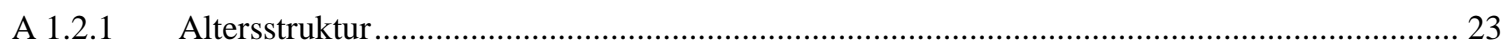

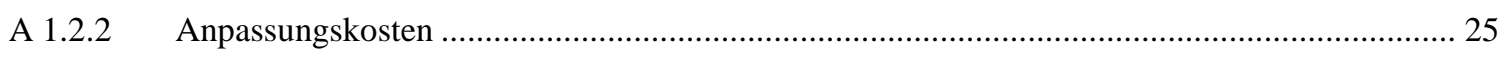

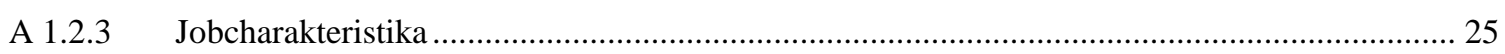

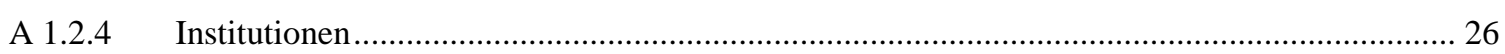

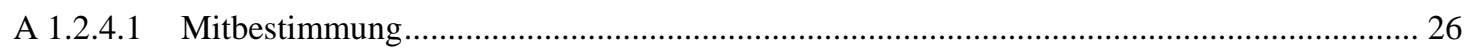

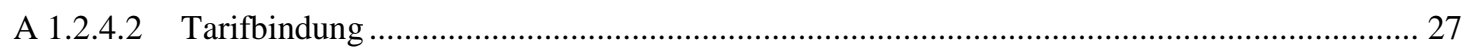

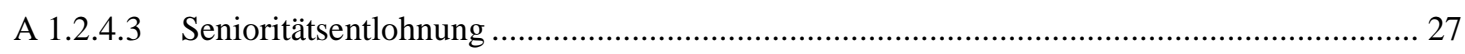

A 1.2.4.4 Altersdiskriminierung im Kündigungsschutz ............................................................. 29

A 1.2.4.5 Altersdiskriminierung in der Sozialgesetzgebung ...................................................... 29

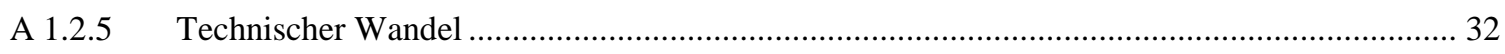

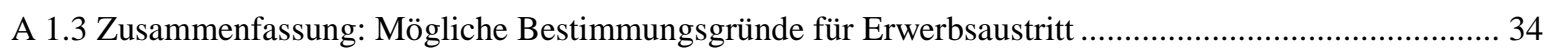

A 2 Datengrundlage Erwerbsaustritt: Linked Employer Employee Datensatz ...................................... 36

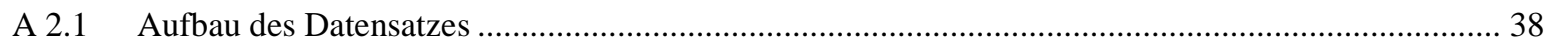

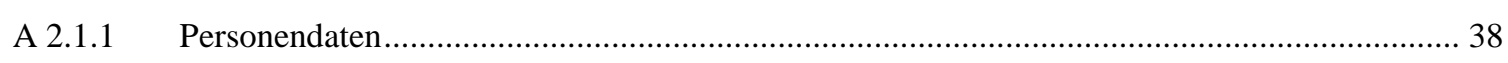

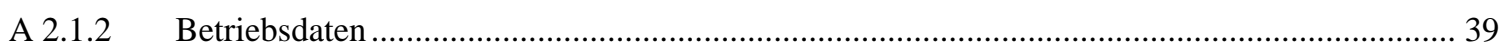

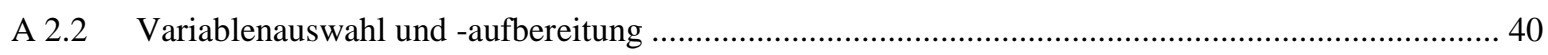

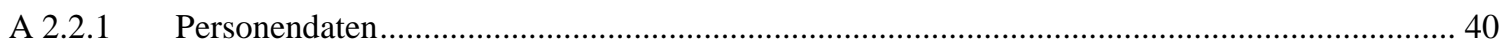

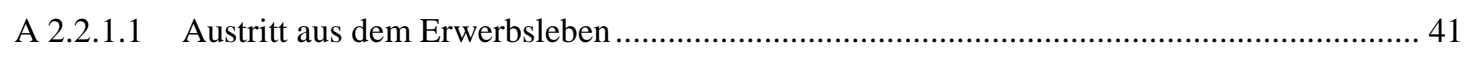

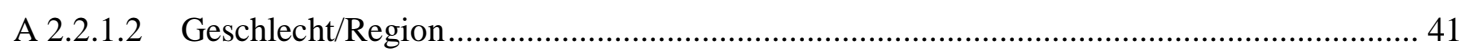




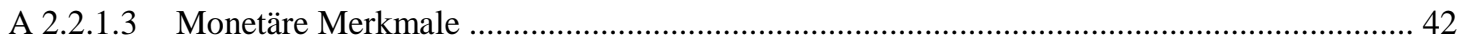

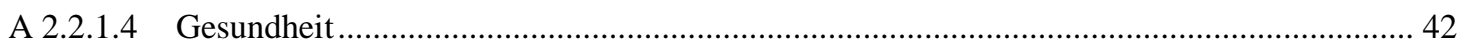

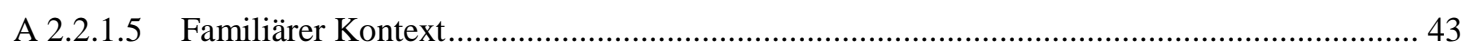

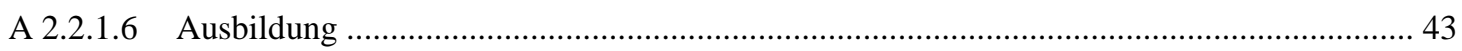

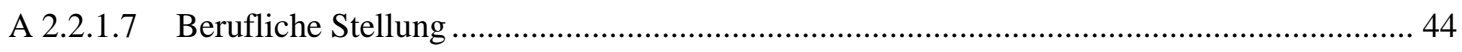

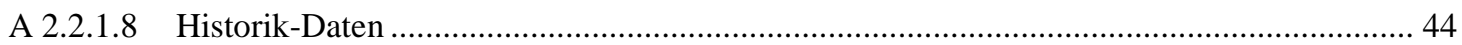

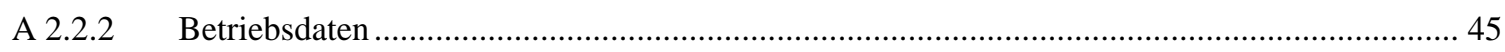

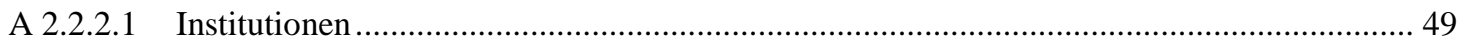

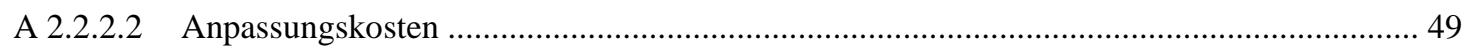

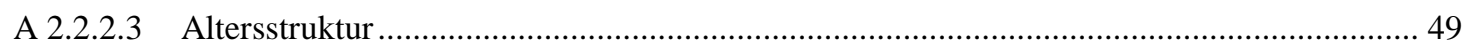

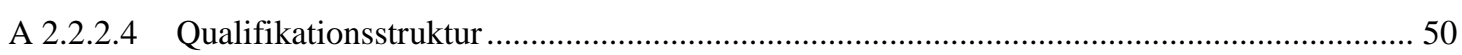

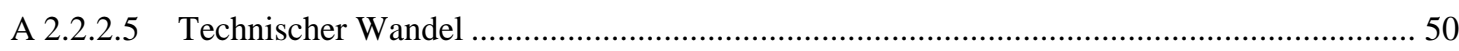

A 2.2.2.6 Sonstige betriebliche Variablen ................................................................................ 50

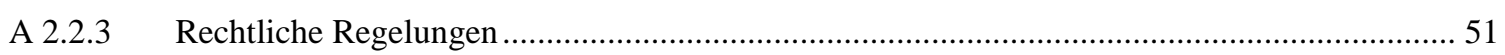

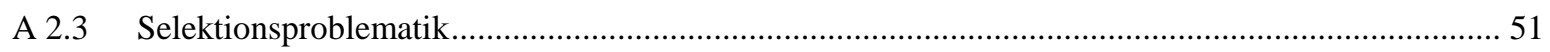

A 2.3.1 Selektion auf Personenebene .......................................................................................... 52

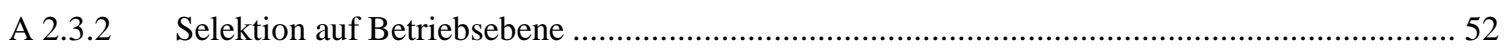

A 2.4 Erwerbsaustritt: Zusammenfassung der Datengrundlage ........................................................... 53

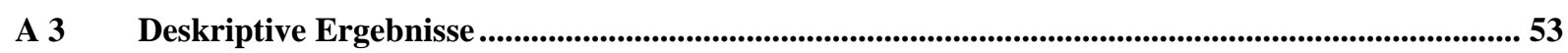

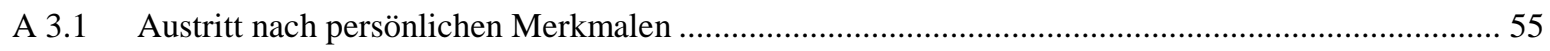

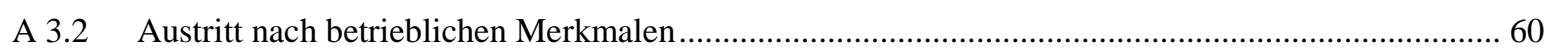

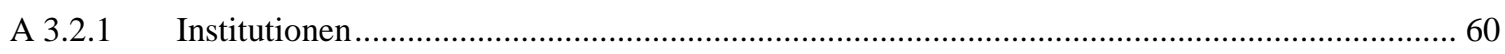

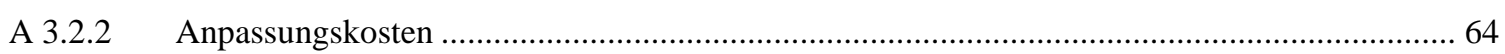

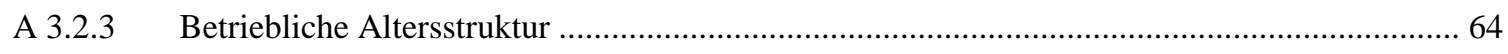

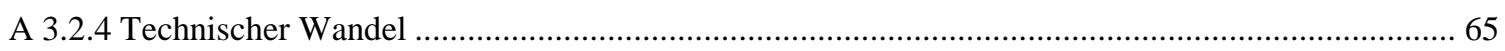

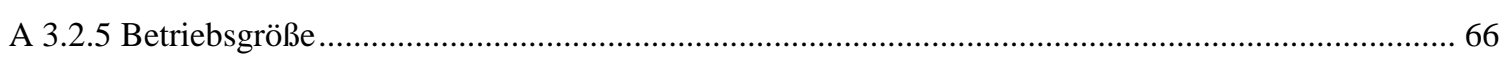

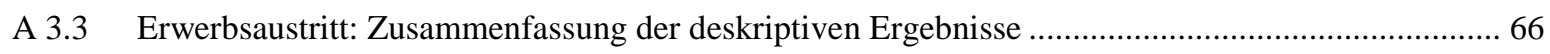

A 4 Mikroökonometrischer Ansatz: Verweildaueranalyse zur Modellierung des Erwerbsaustritts ... 67

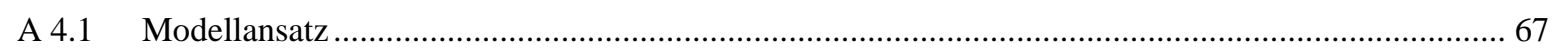

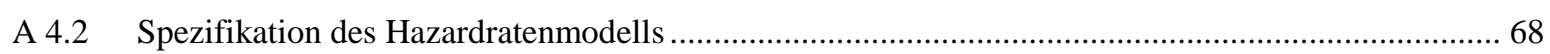

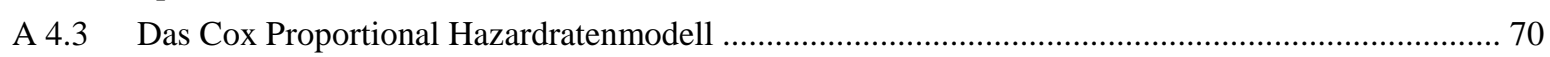

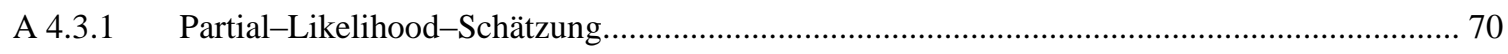

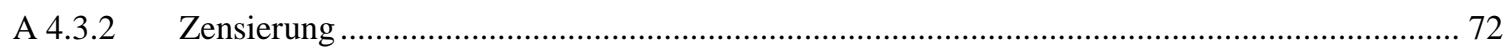

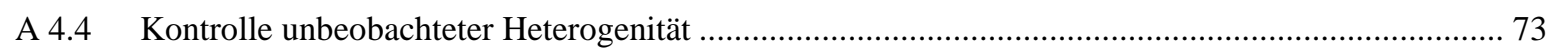

A 5 Mikroökonometrische Ergebnisse: Determinanten des Erwerbsaustritts ........................................ 73

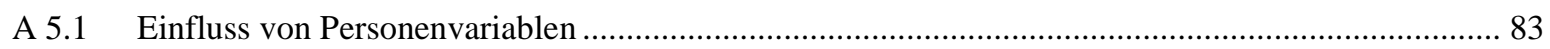

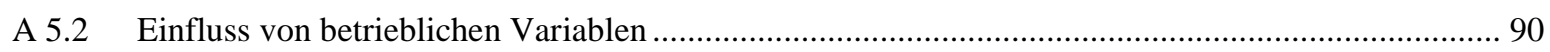

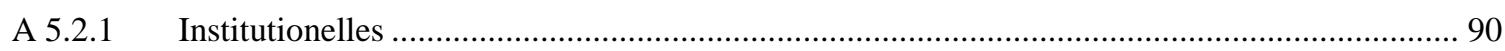

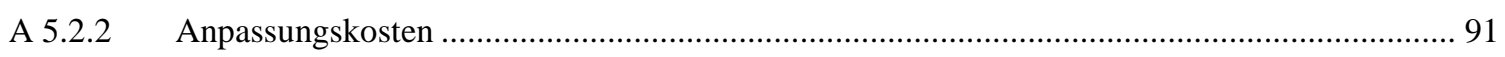

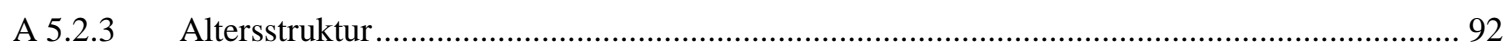

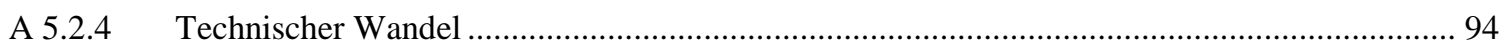

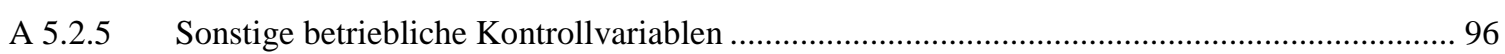

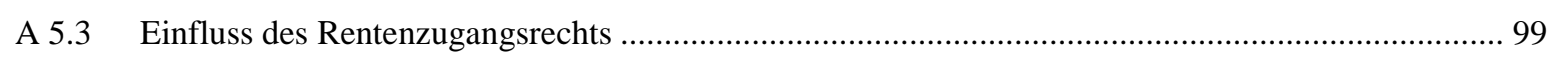


B 1 Kündigungsschutz in Deutschland - institutionelle Hintergründe................................................... 104

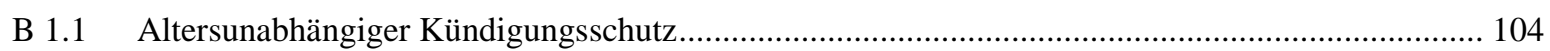

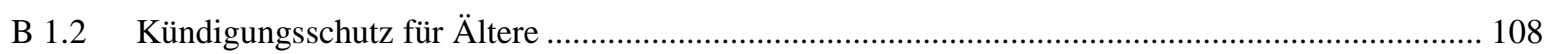

B 2 Datengrundlage Kündigungsschutz: Linked Employer Employee Datensatz................................. 109

B 3 Mikroökonometrischer Ansatz: Das Regression Discontinuity Design.............................................. 110

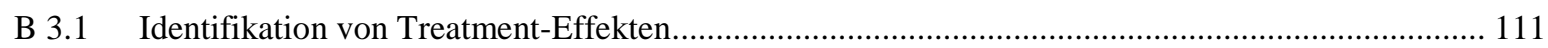

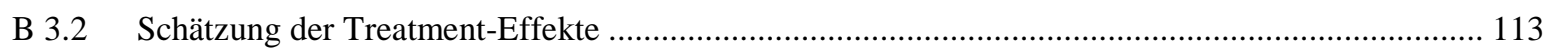

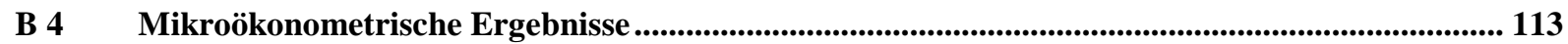

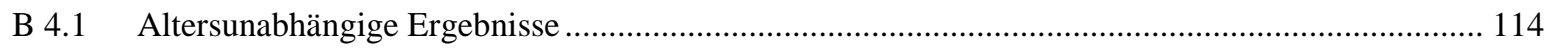

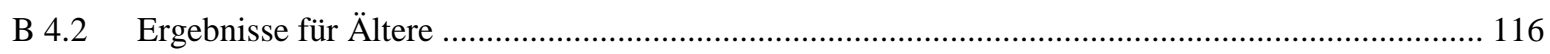

B 5 Zusammenfassung: Einstellungschancen von Älteren - wie wirkt der Schwellenwert im Kündigungsschutzgesetz

\section{BETRIEBLICHE WEITERBILDUNG UND BETRIEBLICHE}

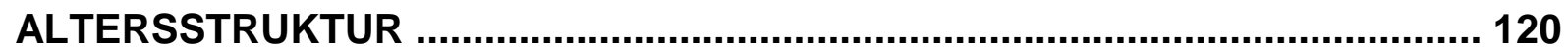

C 1 Wirkungen betrieblicher Weiterbildung in der Diskussion .................................................................. 120

C 2 Datengrundlage Weiterbildung: Linked Employer Employee Datensatz ….................................... 122

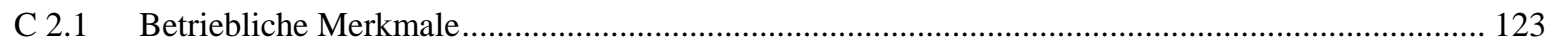

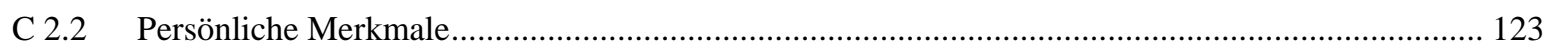

C 3 Mikroökonometrischer Ansatz: Die Matching-Methode ................................................................. 124

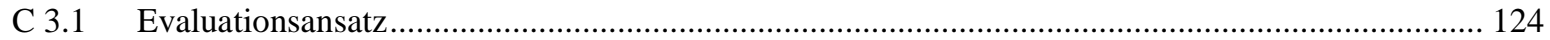

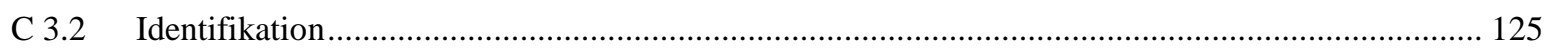

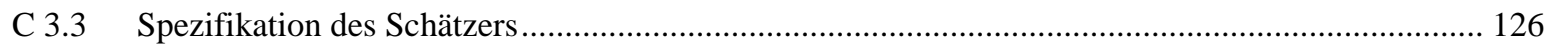

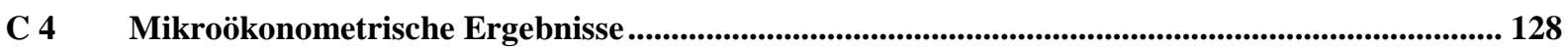

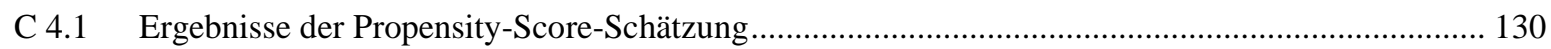

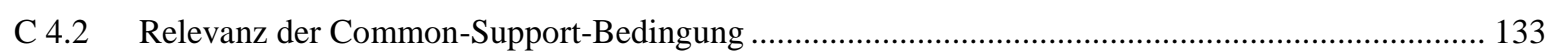

C 4.3 Schätzungen des kausalen Effektes betrieblicher Weiterbildung ..................................................... 133

C 5 Zusammenfassung: Wirkung betrieblicher Weiterbildung auf die Altersstruktur......................... 135

ZUSAMMENFASSUNG UND AUSBLICK ..................................................... 137

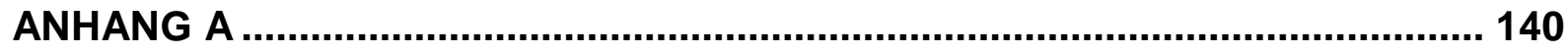

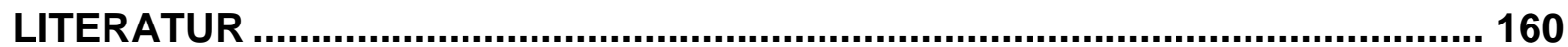




\section{Tabellenverzeichnis}

Tab. 1: Durchschnittliches Renteneintrittsalter nach Geschlecht und Rentenart von 1996 bis 2007

Tab. 2: Zugangsarten zur Altersrente: Regelungen zu Beginn und Änderungen im Beobachtungszeitraum

Tab. 3: Zugänge in die Versichertenrenten in \% nach Jahren

Tab. 4: Zusammenfassung erwarteter Wirkungen auf Erwerbsaustritt

Tab. 5: $\quad$ Kontinuität von Variablen im Verlauf des Betriebspanels 1996 bis 2004

Tab. 6: Anteil an nicht beobachteten Austritten nach Altersgruppe im Jahr 1996 und Personenvariablen [in \%]

Tab. 7: $\quad$ Tests auf Gleichheit der Survivor-Funktionen nach persönlichen Merkmalen 58

Tab. 8: $\quad$ Tests auf Gleichheit der Survivor-Funktionen nach betrieblichen Merkmalen 61

Tab. 9: Cox proportional hazard rate- Schätzungen - Westdeutschland 75

Tab. 10: Cox proportional hazard rate- Schätzungen - Ostdeutschland 79

Tab. 11: Zusammenfassung: Betriebliche Auswirkungen auf Erwerbsaustritt 100

Tab. 12: LATE Schätzungen des Beschäftigungswachstums; Outcome-Variable: Beschäftigungswachstum von 6/96 bis 6/97 und von 6/96 bis 6/98

Tab. 13: LATE Schätzungen des Beschäftigungswachstums; Outcome-Variable: Beschäftigungswachstum von 6/98 bis 6/99 und von 6/98 bis 6/01

Tab. 14: LATE Schätzungen des Beschäftigungswachstums; Outcome-Variable: Beschäftigungswachstum von 6/98 bis 6/02 und von 6/98 bis 6/03

Tab. 15: LATE Schätzungen der Anzahl der Einstellungen von 50-Jährigen und Älteren von 6/96 bis 6/97 und von 6/96 bis 6/98

Tab. 16: LATE Schätzungen der Anzahl der Einstellungen von 50-Jährigen und Älteren von $6 / 98$ bis $6 / 99$ und von $6 / 98$ bis $6 / 01$

Tab. 17: LATE Schätzungen der Anzahl der Einstellungen von 50-Jährigen und Älteren von $6 / 98$ bis $6 / 02$

Tab. 18: Probit-Schätzung der Finanzierung von Weiterbildung 1993

Tab. 19: Probit-Schätzung der Finanzierung von Weiterbildung 1997

Tab. 20: $\quad$ Matching-Ergebnisse: Finanzierung betrieblicher Weiterbildung 1993 bis 1995 
Tab. 21: Matching-Ergebnisse: Finanzierung betrieblicher Weiterbildung 1997

Tab. 22: Durchschnittsalter des Austritts aus der Erwerbstätigkeit nach Altersgruppen im Jahr 1996 und Personenvariablen

Tab. 23: Common Support für die ATT-Schätzung durchgängiger betrieblicher Weiterbildung in den Jahren 1993 bis 1995

Tab. 24: Common Support für die ATT-Schätzung betrieblicher Weiterbildung 1997147

Tab. 25: $\quad$ Probit-Schätzung der Finanzierung von externen Kursen 148

Tab. 26: Probit-Schätzung der Finanzierung von internen Kursen 149

Tab. 27: Probit-Schätzung der Finanzierung von Weiterbildung am Arbeitsplatz 150

Tab. 28: Matching-Ergebnisse: Finanzierung betrieblicher Weiterbildung 1997 - externe Kurse

Tab. 29: Matching-Ergebnisse: Finanzierung betrieblicher Weiterbildung 1997 - interne Kurse

Tab. 30: Matching-Ergebnisse: Finanzierung betrieblicher Weiterbildung 1997 Weiterbildung am Arbeitsplatz

Tab. 31: Verringerung der Verzerrung nach PS-Matching: Schätzungen durchgängiger Weiterbildung 1993 - 1995, nearest neighbor-Matching

Tab. 32: Verringerung der Verzerrung nach PS-Matching: Schätzungen durchgängiger Weiterbildung 1993 - 1995, LLR-Matching

Tab. 33: Verringerung der Verzerrung nach PS-Matching: Schätzungen Weiterbildung 1997, NN-Matching

Tab. 34: Verringerung der Verzerrung nach PS-Matching: Schätzungen Weiterbildung 1997, LLR-Matching 


\section{Abbildungsverzeichnis}

Abb. 1: Entwicklung der Beschäftigtenquoten der 55- bis 64- Jährigen in ausgewählten Staaten der EU 15

Abb. 2: Entwicklung der Beschäftigtenquoten der 50- bis 64- Jährigen in Deutschland und der EU 15 nach Geschlecht

Abb. 3: Entwicklung des durchschnittlichen Erwerbsaustrittsalters in ausgewählten Staaten der EU 15

Abb. 4: Anteil der Langzeitarbeitslosen nach Altersgruppen im Jahr 2003 ........................ 7

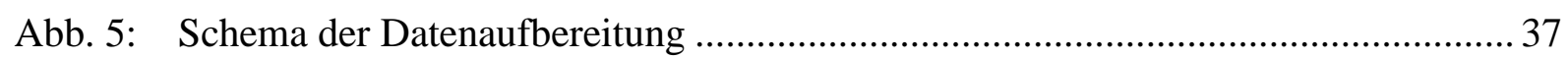

Abb. 6: Anzahl der Spells nach Jahren der Betriebszugehörigkeit im aktuellen Panelbetrieb.

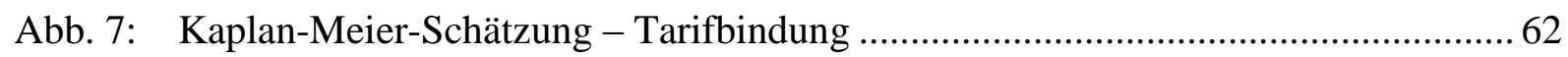

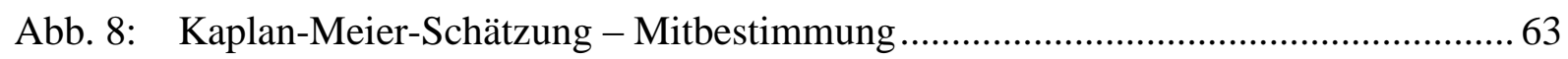

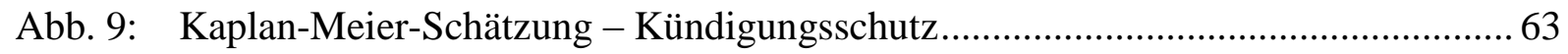

Abb. 10: Kaplan-Meier-Schätzung - Anteil befristet Beschäftigter ...................................... 64

Abb. 11: Kaplan-Meier-Schätzung - Anteil bis-30-jähriger Beschäftigter ........................... 65

Abb. 12: Kaplan-Meier-Schätzung - Investitionen in Kommunikationstechnik .................... 65

Abb. 13: Kaplan-Meier-Schätzung - Betriebsgröße in Anzahl Beschäftigten ....................... 66

Abb. 14: Hazard ratio der Variablen „Aktueller Freistellungsspell“ im Altersprofil ............. 84

Abb. 15: Hazard ratio der Variablen „Anzahl der Freistellungsspells“ nach Ausprägung ..... 85

Abb. 16: Hazard ratio der Variablen Dauer seit Erwerbseintritt nach Ausprägungen ............. 86

Abb. 17: Hazard ratio der Variablen „Dauer der Betriebszugehörigkeit““ in Jahren ............... 87

Abb. 18: Hazard ratio der Variablen „Tagesentgelt in Euro“ im Altersprofil ........................ 89

Abb. 19: Hazard ratio des Anteils Über-50-Jähriger [in \%] nach Alter [in Jahren] ............... 93

Abb. 20: Hazard ratio des Anteils Bis-30-Jähriger [in \%] nach Alter [in Jahren] .................. 93

Abb. 21: Hazard ratio der Variablen ,neue Technik“ .......................................................... 95

Abb. 22: Hazard ratio der Variablen , alte Technik “............................................................ 95

Abb. 23: Anzahl der sozialversicherungspflichtig Beschäftigten nach Betriebsgröße im Juni 2003 . 103

Abb. 24: Zeitliche Entwicklung des Schwellenwertes im KSchG 105 
Abb. 25: Anteile betrieblicher Weiterbildung 1997 nach Art der Weiterbildung............. 128

Abb. 26: Betriebliche Altersstruktur 2004 nach Weiterbildung 1993 bis 1995 ................ 129

Abb. 27: Betriebliche Altersstruktur 2004 nach Weiterbildung 1997 .............................. 129

Abb. 28: Kaplan-Meier-Schätzung nach Geschlecht .................................................. 141

Abb. 29: Kaplan-Meier-Schätzungen nach Region....................................................... 141

Abb. 30: Kaplan-Meier-Schätzungen nach Ausbildung …............................................. 142

Abb. 31: Kaplan-Meier-Schätzung nach beruflicher Stellung ....................................... 142

Abb. 32: Kaplan-Meier-Schätzung nach Eintritt in den Betrieb ...................................... 143

Abb. 33: Kaplan-Meier-Schätzung nach Eintritt in das Erwerbsleben ............................ 143

Abb. 34: Kaplan-Meier-Schätzung nach Klassen von Tagesentgelt................................. 144

Abb. 35: Kaplan-Meier-Schätzungen nach Freistellung in den vergangenen zwei Jahren.....

Abb. 36: Schätzungen des LATE nach Bandbreite .................................................... 145

Abb. 37: Betriebliche Altersstruktur 2004 nach externen Kursen 1997 ........................... 146

Abb. 38: Betriebliche Altersstruktur 2004 nach internen Kursen 1997 .......................... 146

Abb. 39: Betriebliche Altersstruktur 2004 nach WB am Arbeitsplatz 1997..................... 146

Abb. 40: Common Support für die ATT-Schätzung durchgängiger betrieblicher Weiterbildung in den Jahren 1993 bis 1995 .................................................... 147

Abb. 41: Common Support für die ATT-Schätzung betrieblicher Weiterbildung 1997... 147 


\section{Abkürzungsverzeichnis}

\begin{tabular}{|c|c|}
\hline AFG & Arbeitsförderungsgesetz \\
\hline ArbPlSchG & Arbeitsplatzschutzgesetz \\
\hline ATG & Altersteilzeitgesetz \\
\hline BA & Bundesagentur für Arbeit \\
\hline $\mathrm{BeH}$ & Beschäftigten-Historik-Datei \\
\hline BErzGG & Gesetz zum Erziehungsgeld und zur Elternzeit \\
\hline BetrVG & Betriebsverfassungsgesetz \\
\hline BGB & Bürgerliches Gesetzbuch \\
\hline BLH & Beschäftigten-Leistungsempfänger-Historik-Datei \\
\hline CIA & Conditional Independance Assumption \\
\hline CPS & Current Population Survey \\
\hline EntgFG & Entgeltfortzahlungsgesetz \\
\hline FIML & Full Information Maximum Likelihood \\
\hline HRS & Health and Retirement Study \\
\hline IV & Instrumentvariable \\
\hline IAB & Institut für Arbeitsmarkt- und Berufsforschung \\
\hline IABS & IAB Beschäftigtenstichprobe \\
\hline IEBS & Stichprobe der Integrierten Erwerbsbiografien des IAB \\
\hline IKT & Informations- und Kommunikationstechnologie \\
\hline KSchG & Kündigungsschutzgesetz \\
\hline LATE & Local Average Treatment Effect \\
\hline $\mathrm{LeH}$ & Leistungsempfänger-Historik-Datei \\
\hline LIAB & Linked Employer-Employee-Dataset des IAB \\
\hline LIML & Limited Information Maximum Likelihood \\
\hline LLR & Local Linear Regression \\
\hline ML & Maximum Likelihood \\
\hline MSchG & Mutterschutzgesetz \\
\hline $\mathrm{PH}$ & Proportional hazard rate \\
\hline RDD & Regression Discontinuity Design \\
\hline RHS & Retirement History Study \\
\hline SchwbG & Schwerbehindertengesetz \\
\hline SGB & Sozialgesetzbuch \\
\hline SOEP & Sozio-Ökonomisches Panel \\
\hline
\end{tabular}




\section{Einleitung}

Die demographischen Veränderungen der vergangenen Jahre, aber auch der absehbar weiter anstehende Alterungsprozess in vielen Ländern rücken die Gruppe der Älteren in den Mittelpunkt des Interesses der Arbeitsmarktforschung. Ihr zunehmender Anteil an der Erwerbsbevölkerung stellt die Betriebe ebenso wie das Kollektiv der Sozialversicherten vor Herausforderungen. Unternehmen droht in den kommenden Jahren eine Knappheit an ausgebildeten Beschäftigten. Renten-, Kranken- und Pflegeversicherung sind von der Entwicklung in doppelter Hinsicht betroffen: Eine wachsende Zahl von Leistungsbeziehern steht immer weniger Beitragszahlern gegenüber und droht damit die Finanzen der Sicherungssysteme in eine Schieflage zu bringen. Verschärft wurde das Problem zuletzt dadurch, dass sich zur demographischen Entwicklung ein weiterer Trend gesellte, der sich negativ auf die Finanzen der Sozialversicherer auswirkte: Bis in die späten 90er Jahre hinein sank die Beschäftigungsquote der Älteren, ebenso ging das durchschnittliche Renteneintrittsalter zurück, um erst in den vergangenen Jahren wieder leicht anzusteigen. Angesichts dieser Entwicklung sind Ältere als Problemgruppe der Arbeitsmarktpolitik inzwischen auch in den Blickpunkt der Öffentlichkeit gerückt. Diese gesteigerte Wahrnehmung auch von Seiten der Politik findet ihren Ausdruck nicht zuletzt in den Maßnahmen, die speziell auf Ältere und ihren Verbleib im Erwerbsleben ausgerichtet sind. So haben die verschiedenen Bundesregierungen der vergangenen Jahre Programme aufgelegt, die darauf abzielen, Älteren stärker in den Arbeitsmarkt zu integrieren und sie länger in Beschäftigung zu halten.

Dass die Gruppe der Älteren nicht nur interessant, sondern auch für die wissenschaftliche Auswertung empirisch-analytisch greifbar wurde, ist dem Umstand zu verdanken, dass Daten über sie vorliegen. Was trivial klingt, ist keine Selbstverständlichkeit, sondern das Resultat einer Vernetzung verschiedener Datenproduzenten und der Verknüpfung von Datenquellen. Zudem wurden in den vergangenen Jahren erhebliche Anstrengungen zur Bereitstellung solcher Daten zu Forschungszwecken unternommen. Keine der zu dieser Arbeit zusammengestellten Studien wäre meinem direkten Vorgänger an der Universität Lüneburg möglich gewesen. Die dafür notwendigen Daten standen vor wenigen Jahren schlicht nicht zur Verfügung.

Empirisch stehen die verschiedenen Analysen unter dem gemeinsamen Dach der Mikroökonometrie, im Besonderen der Evaluationsmethoden. Hier profitieren die letzten beiden Arbeiten von Weiterentwicklungen und Wiederentdeckungen von Methoden zur Analyse von selbstselektierten Merkmalen und zur Behandlung der auch daraus resultierenden Endogenitätsproblematik.

All diese drei Entwicklungen werden in dieser Arbeit aufgegriffen. Inhaltlich beschäftigen sich alle Teile mit der Frage, wie und im Besonderen unter welchen betrieblichen Umständen es möglich ist, Ältere wieder zu Arbeit zu verhelfen oder - je nach Erwerbsstatus - sie in Be- 
schäftigung zu halten. Alle Arbeiten sind empirischer Natur, fußen in unterschiedlichem Ausmaß sowohl auf Betriebs- als auch auf Personeninformationen und bedienen sich des Instrumentariums der Mikroökonometrie. ${ }^{1}$

Nicht zuletzt reiht sich die Arbeit thematisch ein in die Erforschung der Stellung Freier Berufe in Wirtschaft und Gesellschaft, der sich das Lüneburger Institut widmet, an dem die Arbeit entstanden ist. Auch wenn nicht immer explizit erwähnt, werden die Freien Berufe mit der Berücksichtigung und speziellen Beachtung von Betriebsgrößenklassen, aber auch Branchenangaben untersucht. Damit beleuchtet die Arbeit mit der Rolle als mögliche Arbeitgeber und Weiterbilder auch Älterer eine andere Seite der Freien Berufe und ergänzt damit die bisherigen Studien und Dissertationen am Forschungsinstitut Freie Berufe.

Nach einer Einführung in das Thema folgen drei Kapitel, die dem Thema „Übergänge auf dem Arbeitsmarkt für Ältere“ zuzuordnen sind. In Kapitel A beschäftige ich mich mit der Frage, welche insbesondere betrieblichen Bestimmungsgründe für den Austritt aus dem Erwerbsleben verantwortlich sind. Kapitel B geht dann näher der Frage nach, wie sich das deutsche Kündigungsschutzrecht und sein Schwellenwert auf die Beschäftigungschancen von Älteren auswirken. Anlass ist die Vermutung, dass potentielle Arbeitgeber von der Einstellung gerade Älterer absehen, weil diese unter einem besonderen Schutz im Entlassungsfall stehen. Kapitel C greift einen weiteren losen Faden des ersten Kapitels auf und untersucht, wie sich betriebliche Weiterbildung in der Altersstruktur der Betriebe niederschlägt. Ein Abschlusskapitel fasst die Ergebnisse zusammen und wagt einen Ausblick.

Zum Einstieg allerdings sollen einige Statistiken einen Überblick über das Thema „Arbeitsmarkt für Ältere“ geben. Dabei sind die Altersprofile von Erwerbsbeteiligung und das Alter bei Erwerbsaustritt von Interesse, aber auch der internationale Vergleich, der Möglichkeiten für den deutschen Teilarbeitsmarkt der Älteren aufzeigt. Anhand einiger „stylized facts“ soll sowohl die gegenwärtige Situation dieser Generation näher beschrieben werden, als auch die Entwicklung des Arbeitsmarktes für Ältere seit Mitte der 1990er Jahre: Die zeitliche Abgrenzung ergibt sich einerseits aus dem beschriebenen Paradigmenwechsel in der Arbeitsmarktund Rentenpolitik, andererseits weil für die anschließende empirische Untersuchung ein geeigneter Datensatz erst ab 1996 vorliegt. $^{2}$

Am anschaulichsten lässt sich die Erwerbssituation der älteren Erwerbspersonen anhand der Beschäftigtenquoten darlegen. Sie setzen die Zahl der Beschäftigten ins Verhältnis zur Wohnbevölkerung und bleiben damit weitgehend unberührt von Entwicklungen der Sozialpo-

\footnotetext{
${ }^{1}$ Alle Ergebnisse und Auswertungsprogramme, die zumeist als Stata-.do-Files vorliegen, lasse ich anderen Forscherinnen und Forschern auf Anfrage gern zukommen. Alle verwendeten Daten sind auf entsprechenden Antrag den Kolleginnen und Kollegen im Forschungsdatenzentrum des Instituts für Arbeitsmarkt- und Berufsforschung (IAB) zugänglich.

${ }^{2}$ Einen Überblick über die Erwerbssituation von Älteren in den 80er und frühen 90er Jahren beschreiben beispielsweise die Arbeiten von Hoffmann (1996) und Thon (1996).
} 
litik und ihren Definitionen einzelner Gruppen auf dem Arbeitsmarkt. Statistiken zu altersspezifischen Arbeitslosenquoten beispielsweise können die Arbeitsmarktposition nur bedingt beschreiben, da diese auch die Entwicklung der Erwerbspersonen generell wiedergibt und somit z.B. stark von den Zugangsregelungen zu Invaliditätsrenten abhängt (vgl. Funk (2004), S. 23f., Koller (2001), S. 16f.).

Abb. 1: Entwicklung der Beschäftigtenquoten der 55- bis 64- Jährigen in ausgewählten Staaten der EU 15

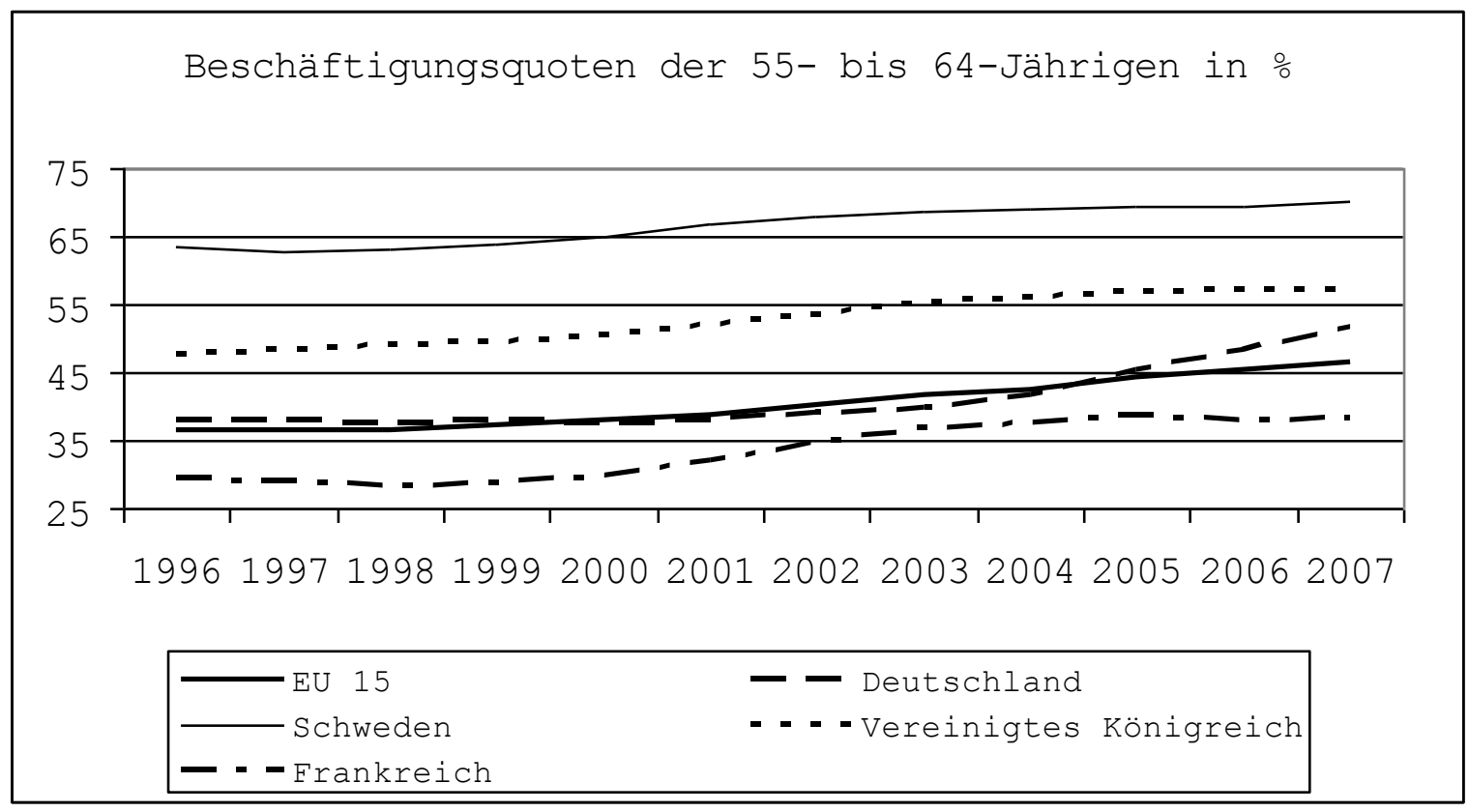

Quelle: Eurostat (2008)

Wie aus Abb. 1 ersichtlich, ist die Erwerbsbeteiligung Älterer in Deutschland im internationalen Vergleich gering und erst in den vergangenen Jahren angestiegen: Nach der von Eurostat ausgewiesenen Beschäftigtenquote ${ }^{3}$ aus dem Jahr 2007 übertrifft die Bundesrepublik mit 51,5 Prozent zwar den Durchschnittswert der EU-15-Staaten (2007: 46,4 Prozent) inzwischen deutlich. Auch sind die Fortschritte in der Integration Älterer hierzulande größer als in den europäischen Nachbarländern, wie der Vergleich der Entwicklung der Beschäftigungsquoten in den vergangenen Jahren zeigt. Allerdings kommen die skandinavischen Länder wie hier exemplarisch Schweden mit rd. 70 Prozent auf weit höhere Werte. Auch in Großbritannien oder den Werten von Irland und Portugal (hier nicht dargestellt) - ist ein weit größerer Anteil der 55- bis 64-Jährigen noch in Beschäftigung. Schwieriger als in Deutschland sieht dagegen die Lage für Ältere auf den Arbeitsmärkten in den osteuropäischen Staaten aus. Bis auf die

\footnotetext{
${ }^{3}$ Die Quote ergibt sich als Division der Anzahl von erwerbstätigen Personen im Alter zwischen 55 und 64 Jahren durch die Gesamtbevölkerung derselben Altersklasse. Der Indikator bezieht sich auf die EGArbeitskräfteerhebung. Dabei fassen die EU-Statistiker die erwerbstätige Bevölkerung recht weit: Es zählt dazu, wer während der Referenzwoche irgendeine Tätigkeit gegen Entgelt mindestens eine Stunde ausgeübt hat oder nur vorübergehend vom Arbeitsplatz abwesend war.
} 
baltischen Länder erreichen alle osteuropäischen Beitrittsländer zur Union weit unterdurchschnittliche Beschäftigungsquoten unter den Älteren. Aber auch in den südeuropäischen Ländern Italien, Spanien und Griechenland, sowie dem exemplarisch aufgenommenen Frankreich ist der Beschäftigtenanteil in der untersuchten Altersgruppe deutlich geringer als im Durchschnitt der EU-15-Staaten.

Eine nahe liegende, wenn auch bei weitem nicht die einzige Erklärung für den skizzierten Anstieg in Deutschland bietet das Nachwachsen von weiblichen Kohorten, die eine höhere Erwerbsbeteiligung aufweisen als ihre Vorgängerinnengeneration. Zwar zeigt Abb. 2, dass die Quote der Frauen im Beobachtungszeitraum von 28,2 Prozent auf 43,6 Prozent um gut 15 Prozentpunkte steigt. Gleichzeitig lässt sich aber auch für die Männer ein deutlich höherer Anteil ausmachen: von 1996 bis 2007 stieg der Anteil der Beschäftigten an der männlichen Wohnbevölkerung in dieser Altersgruppe um immerhin knapp 12 Prozentpunkte von 47,8 Prozent auf 59,7 Prozent. Unabhängig vom Geschlecht hat sich die Integration von älteren Menschen in den Arbeitsmarkt in den vergangenen Jahren deutlich verbessert.

Abb. 2: $\quad$ Entwicklung der Beschäftigtenquoten der 50- bis 64- Jährigen in Deutschland und der EU 15 nach Geschlecht

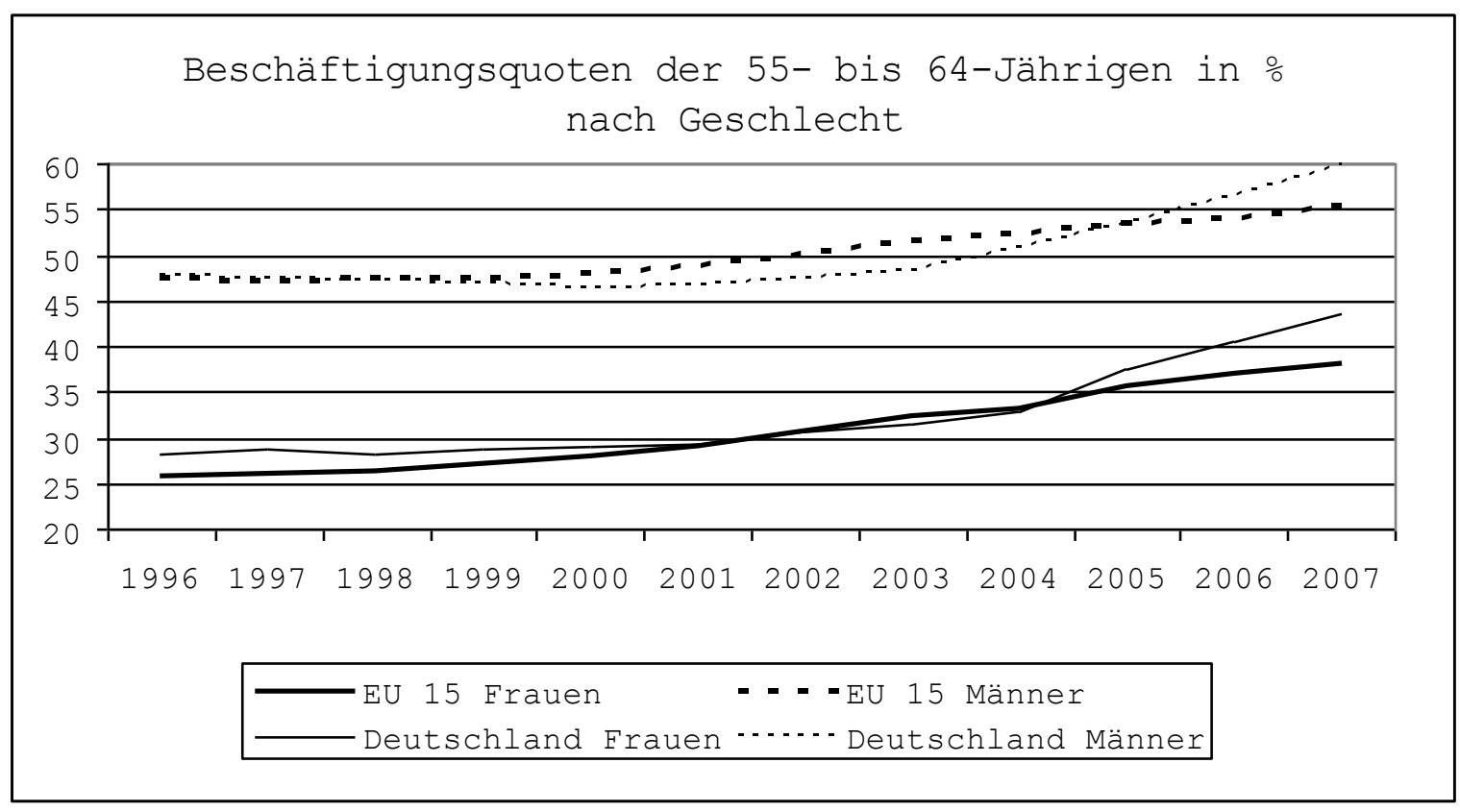

Quelle: Eurostat (2008)

Die Entwicklung der Arbeitsmarktintegration Älterer in den vergangenen Jahren findet ihren Niederschlag auch im Zeitpunkt des Erwerbsaustritts: Er weist in den verglichenen Ländern eine ähnliche Entwicklung auf wie die eben beschriebene. In den vergangenen Jahren hat sich das Alter beim Austritt aus dem Erwerbsleben in allen untersuchten Ländern deutlich erhöht. In Deutschland stieg es von 60,6 Jahren im Jahr 2001 auf 61,9 Jahre im letzten verfügbaren Beobachtungszeitraum 2006. Für die EU-15-Staaten fiel die Entwicklung von 60,3 auf 61,6 Jahre ähnlich aus. Auch im internationalen Vergleich wiederholt sich der Befund: Zwar lassen 
sich bei diesem Indikator Fortschritte bei der Integration Älterer in den deutschen Arbeitsmarkt erkennen. Auch fällt der internationale Vergleich mit der Gesamtheit der westeuropäischen Staaten der EU-15 positiv aus. Dennoch sind andere Länder - hier ist z.B. wieder Skandinavien zu nennen - erfolgreicher darin, Ältere lange in Beschäftigung zu halten. So sind Schweden, wenn sie den Arbeitsmarkt verlassen, im Durchschnitt fast 64 Jahre alt, und auch Britinnen und Nordiren kommen auf ein Durchschnittsalter von über 63 Jahren bei der jüngsten Schätzung (vgl. Abb. 3).

Abb. 3: $\quad$ Entwicklung des durchschnittlichen Erwerbsaustrittsalters in ausgewählten Staaten der EU 15

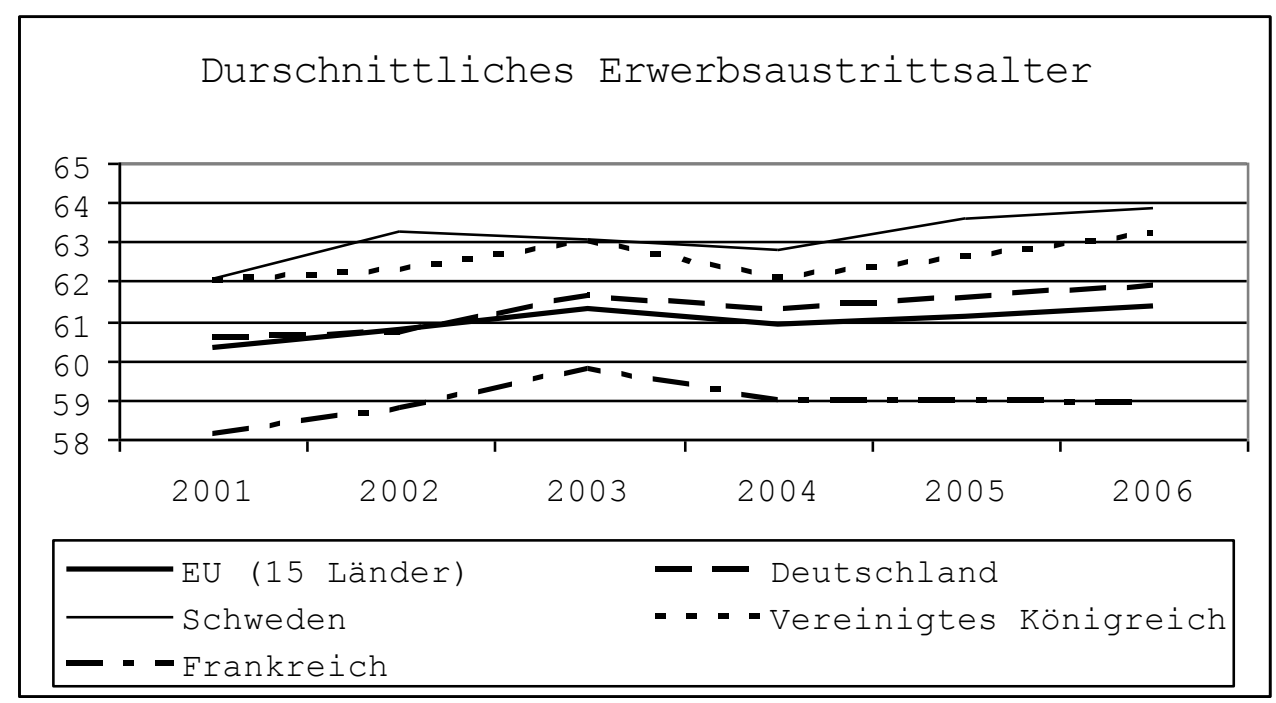

Quelle: Eurostat (2008)

Eng verknüpft mit dem Erwerbsaus- ist der Renteneintritt. Allerdings spiegelt er - mehr noch als das erste Ereignis - die Entwicklungen im Rentenzugangsrecht und damit die politische Einflussnahme auf den Arbeitsmarkt für Ältere wider. Nachdem das durchschnittliche Zugangsalter in die Alters- und Erwerbsunfähigkeitsrenten bis zum Jahr 1997 auf einen Tiefstwert von 59,6 Jahre gesunken war, stiegt der Wert seitdem Jahr für Jahr langsam, aber stetig und lag im Jahr 2006 bei 60,9 Jahren (vgl. Tab. 1). Die Entwicklung ist für Frauen wie Männer gleichermaßen zu beobachten, wobei Männer inzwischen ihre Rente im Durchschnitt später als Frauen antreten.

Allerdings muss angemerkt werden, dass das durchschnittliche Renteneintrittsalter eine wenig verlässliche Größe zur Bestimmung des Zugangsverhaltens in die Rente ist. So können unterschiedliche Kohortengrößen der relevanten Jahrgänge einen bedeutenden Einfluss auf diese Maßgröße ausüben. Zudem bilden Erwerbsminderungsrenten in frühem Alter nicht das Erwerbsverhalten der untersuchten Gruppe ab. Büttner und Knuth (2004, S. 11 f.) berichten, dass sich das Eintrittsalter auf durchschnittlich 60,9 im Jahr 1996 und 61,9 2003 erhöht, wenn man Zugänge in Erwerbsminderungsrenten von Personen unter 50 Jahren ausschließt. 
Tab. 1: Durchschnittliches Renteneintrittsalter nach Geschlecht und Rentenart von 1996 bis 2007

\begin{tabular}{ccccccccccccc}
\hline & \multicolumn{3}{c}{ Alle Versichertenrenten } & \multicolumn{3}{c}{ Erwerbsminderungsrenten } & \multicolumn{3}{c}{ Altersrenten } \\
& alle & $\%$ & Männer & Frauen & alle & $\%$ & M & F & alle & $\%$ & M & F \\
\hline \hline 1996 & 59,7 & 100 & 59,2 & 60,2 & 51,9 & 24,7 & 52,6 & 50,8 & 62,3 & 75,3 & 62,1 & 62,4 \\
1997 & 59,6 & 100 & 59,2 & 60,1 & 51,7 & 24,0 & 52,4 & 50,5 & 62,1 & 76,0 & 62,0 & 62,3 \\
1998 & 59,7 & 100 & 59,3 & 60,2 & 51,5 & 22,6 & 52,3 & 50,3 & 62,2 & 77,4 & 62,1 & 62,2 \\
1999 & 60,1 & 100 & 59,6 & 60,5 & 51,6 & 19,9 & 52,3 & 50,5 & 62,2 & 80,1 & 62,1 & 62,2 \\
2000 & 60,2 & 100 & 59,8 & 60,5 & 51,4 & 19,6 & 52,2 & 50,3 & 62,3 & 80,4 & 62,2 & 62,3 \\
2001 & 60,2 & 100 & 59,9 & 60,5 & 51,0 & 19,7 & 51,7 & 50,0 & 62,4 & 80,3 & 62,4 & 62,5 \\
2002 & 60,4 & 100 & 60,1 & 60,7 & 50,4 & 18,6 & 51,1 & 49,5 & 62,7 & 81,4 & 62,6 & 62,8 \\
2003 & 60,7 & 100 & 60,5 & 60,9 & 50,1 & 17,4 & 50,7 & 49,2 & 62,9 & 82,6 & 62,9 & 62,9 \\
2004 & 60,8 & 100 & 60,5 & 61,0 & 49,8 & 17,3 & 50,4 & 49,1 & 63,1 & 82,7 & 63,1 & 63,0 \\
2005 & 60,8 & 100 & 60,7 & 61,0 & 49,9 & 17,5 & 50,5 & 49,2 & 63,2 & 82,5 & 63,1 & 63,2 \\
2006 & 60,9 & 100 & 60,8 & 61,1 & 50,0 & 17,4 & 50,5 & 49,3 & 63,2 & 82,6 & 63,3 & 63,2 \\
2007 & 60,7 & 100 & 60,8 & 60,6 & 50,0 & 18,7 & 50,5 & 49,3 & 63,1 & 81,3 & 63,3 & 63,0 \\
\hline
\end{tabular}

Quelle: DRV Bund (2008), eigene Erstellung

Zusammenfassend lässt sich trotz der genannten Einschränkungen erkennen, dass in den späten 1990er Jahren eine Trendwende eingesetzt hat, die die bis dahin langfristige Tendenz eines immer früheren Renteneintritts und damit Erwerbsaustritts umkehrte. Bei genauerer Betrachtung der Rentenarten in Tab. 1 fallen zwei Entwicklungen bei dem seit 1997 beobachteten Anstieg besonders auf. So ging einerseits der Anteil der Eintritte in die Rente wegen Erwerbsunfähigkeit seit 1996 stark zurück. Machten Rentner dieser Rentenart, die häufig in jungen Jahren Ansprüche anmelden, zum Beobachtungsbeginn noch knapp ein Viertel der Renteneintritte aus, lag ihr Anteil im Jahr 2007 bei nur noch 18,7 Prozent. Allerdings wird der Effekt dadurch verwässert, dass Neurentner wegen Erwerbsunfähigkeit im Mittel früher den Ruhestand antreten. Ein zweiter Grund für den Anstieg ist ein späterer Eintritt der Altersneurentner. Männer dieser Kategorie gingen 2007 anderthalb Jahre später in Altersrente als noch 1997. Für Frauen beträgt der Unterschied rd. sechs Monate.

Allein diese wenigen Daten verdeutlichen, dass die Entscheidung über den Verbleib im Erwerbsleben eine Frage der Alternativen ist. Die Verschärfung der Regelungen im Rentenzugangsrecht mag in den vergangenen Jahren den Anreiz verringert haben, aus einem bestehenden Beschäftigungsverhältnis vorzeitig in den Ruhestand zu gehen. Gleichzeitig stellt sich die Frage, ob es Älteren ohne Job gelingt, sich wieder in das Arbeitsleben zu integrieren. Viele Studien, die sich mit der Arbeitslosigkeitsdauer beschäftigen, kommen einhellig zu dem Ergebnis, dass mit zunehmendem Alter die erwartete Dauer der Arbeitslosigkeit steigt und damit die Wiedereinstellungschancen sinken. ${ }^{4}$

Auch in diesen Bemühungen scheinen andere europäische Staaten erfolgreicher zu sein. Betrachtet man den Anteil der Langzeitarbeitslosen an den Arbeitslosen insgesamt in einzelnen

\footnotetext{
${ }^{4}$ Vgl. z.B. Fitzenberger und Wilke (2004), Wilke (2005) oder Frosch (2007).
} 
Ländern (vgl. Abb. 4), so fällt auf, dass nicht nur die Gesamtquote in den Vergleichsländern deutlich geringer ist. Auch in der Gruppe der Über-50-Jährigen liegt Deutschland mit einer Quote von über 64 Prozent an der Spitze dieser Länderauswahl. Diese hohe Anzahl an Arbeitslosen ist ein deutlicher Hinweis, dass auch die Nachfrage restringierend auf den Teilarbeitsmarkt der Älteren wirkt.

Abb. 4: $\quad$ Anteil der Langzeitarbeitslosen nach Altersgruppen im Jahr 2003

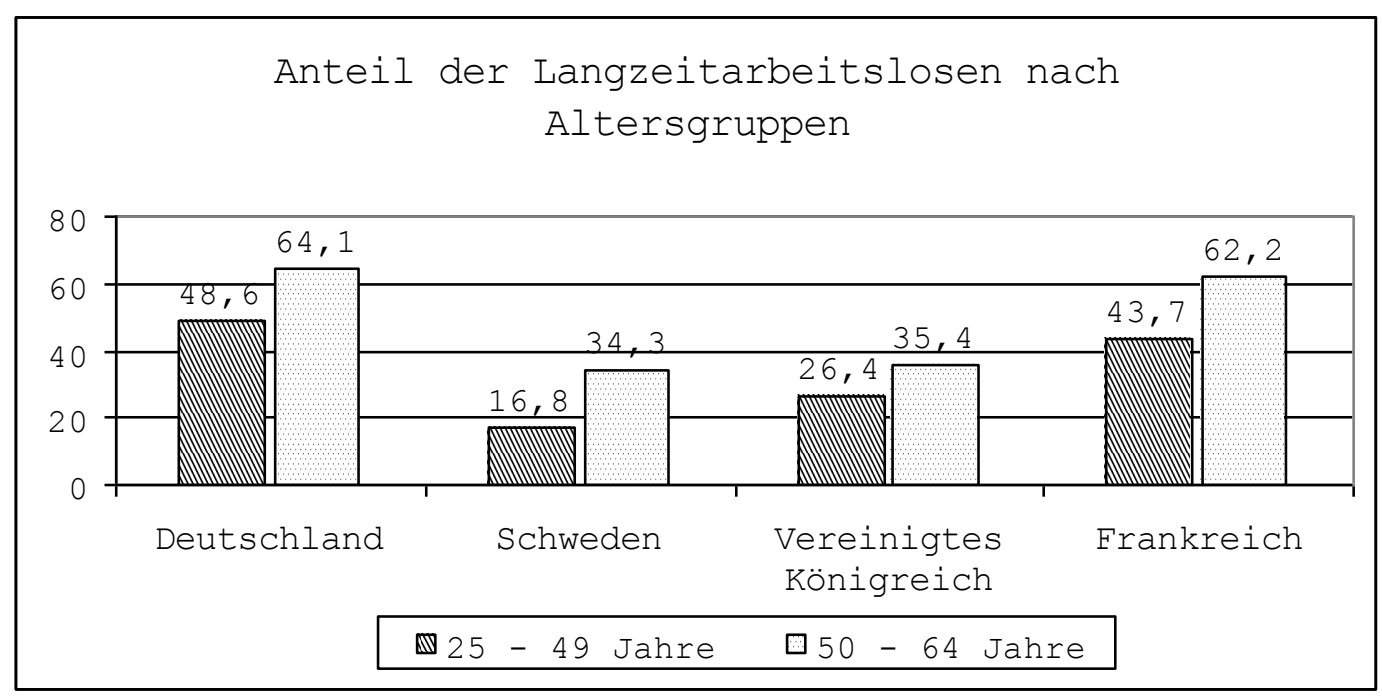

Quelle: OECD (2005)

Dies mag nicht nur an der mangelnden Nachfrage nach Älteren liegen, sondern auch an gängigen Zugangswegen in den Ruhestand und geringeren Anforderungen, die älteren Arbeitslosen auferlegt werden. Dennoch unterstreicht es, dass die Rolle der Betriebe und ihr Bedarf an älteren Beschäftigten eine nicht unwesentliche Rolle in den Übergangsprozessen auf dem Arbeitsmarkt für Ältere spielt. Diese Rolle zu beleuchten ist Gegenstand der vorliegenden Arbeit. 


\section{A Austritt aus dem Erwerbsleben: eine Mikroanalyse der betrieblichen Einflüsse ${ }^{5}$}

Vor dem demografischen Hintergrund liegt die Forderung nahe, die Finanzierungsprobleme der Sozialversicherungen zu lösen, indem man das Erwerbsaustritts- und das Renteneintrittsalter anhebt. Gelingt es, diese Übergänge zu verschieben, verringert sich zum einen die erwartete Dauer der Zahlungen an die nun zudem später in die Rente eintretenden Versicherten, zum anderen steht die Person damit länger dem Arbeitsmarkt als potentieller Beitragszahler zur Verfügung. ${ }^{6}$ Mit den seit Jahren vorgenommenen Änderungen am Rentenrecht, die einerseits die absehbar schwierige Situation der kommenden Jahre reflektieren, und andererseits mit der angesprochenen Lenkungsabsicht einer Verzögerung der Erwerbsaustrittsentscheidung begründet werden, haben die Sozialpolitiker bereits weit reichende Maßnahmen ergriffen, die an der Arbeitsangebotsseite ansetzen.

In diesem Kapitel A soll untersucht werden, welchen Einfluss die Betriebe auf die Erwerbsbeteiligung Älterer ausüben. Eine empirische Analyse für die Jahre 1996 bis 2004 soll aufzeigen, welche betrieblichen Faktoren neben den Anreizen des Rentenrechts und persönlichen Merkmalen den Austrittszeitpunkt aus dem Erwerbsleben beeinflussen. Kapitel A ist wie folgt aufgebaut: Zunächst werden theoretische Vorüberlegungen über die Bestimmungsgründe für die Erwerbs- und Verrentungsentscheidung Älterer angestellt und in die bestehende Literatur zu dem Thema eingebunden. Spezielles Augenmerk gilt dabei Fragen, die in bisherigen empirischen Untersuchungen zum Thema nur am Rand behandelt wurden: Wie wirkt der beschäftigende Betrieb mit seiner Qualifikations- und Altersstruktur, aber auch seine technische Ausstattung und seine institutionellen Restriktionen wie Tarifbindung und das Vorhandensein eines Betriebsrates auf den Verbleib Älterer in Beschäftigung? Der dann folgende Abschnitt stellt den verwendeten Datensatz, die notwendigen Schritte der Datenaufbereitung und die enthaltenen Variablen im Detail vor und diskutiert ihre Tauglichkeit für die anschließenden Analysen. Bevor die Ergebnisse der ökonometrischen Verweildaueranalyse vorgestellt und erörtert werden, soll das verwendete proportionale Hazardratenmodell mit seinen Annahmen

\footnotetext{
${ }^{5}$ Die diesem Kapitel zugrunde liegenden Arbeiten wurden finanziert vom Forschungsnetzwerk Alterssicherung (FNA) der Deutschen Rentenversicherung. Die Ergebnisse wurden diskutiert auf einem Workshop des FNA sowie im Forschungskolloquium Freie Berufe, Mittelstand und empirische Wirtschaftsforschung an der Universität Lüneburg. Der Endbericht des Projektes "Betriebliche Einflüsse auf die Wahl des Renteneintrittsalters“ bildet die Grundlage dieses Kapitels.

${ }^{6}$ Für die gesetzliche Regelaltersrente gilt zwar keine Hinzuverdienstgrenze und ihr Bezug ist damit nicht rechtlich an den Rückzug aus dem Erwerbsleben gebunden, in der Praxis geht der Renteneintritt aber i.d.R. einher mit dem Ende einer aktiven Teilnahme am Arbeitsmarkt. Zudem hat der Gesetzgeber für die meisten Bezieher von vorgezogenen Altersrenten, die das Renteneintrittsgeschehen in Deutschland bestimmen, die Möglichkeit des Hinzuverdienstes stark beschränkt.
} 
und seiner Eignung für das spezielle Auswertungsziel formal eingeführt und diskutiert werden. Den Abschluss bilden eine Zusammenfassung der Ergebnisse und ein Ausblick auf mögliche Erweiterungen der Arbeit.

\section{A 1 Theoretische Grundlagen: Erwerbsaustritt in der Diskussi- on}

Die Frage nach dem Erwerbsverhalten Älterer ist in Deutschland in den vergangenen Jahren recht ausgiebig untersucht worden. Neben den Studien von Schmidt (1995), Siddiqui (1997), Börsch-Supan und Schnabel (1998a und 1998b) und Antolin und Scarpetta (1998), die sich vorrangig mit Anreizen des deutschen Rentenrechts und ihren Auswirkungen auf das Verrentungsverhalten hierzulande beschäftigen, haben seit Anfang der 80er Jahre viele Studien um die Erklärung des faktischen Renteneintrittsalters gekreist. Keine dieser Studien bezieht allerdings - außer groben Kategorien der Sektorzugehörigkeit - betriebliche Merkmale in die Analyse ein.

Bei der Klärung der Frage nach Gründen für den Austritt aus dem Erwerbsleben stehen sich Theorien der individuellen Arbeitsangebots- und betrieblichen Nachfrageentscheidung gegenüber. Zudem sind beide Seiten in ihrem Verhalten nicht frei, sondern an institutionelle Regeln gebunden, wie etwa die Zugangsbestimmungen zur Rente oder der besondere Kündigungsschutz älterer Beschäftigter. Eingebunden in die entsprechende Literatur sollen im Folgenden theoretische Überlegungen angestellt werden, welche Faktoren in welcher Weise auf die Verrentungsentscheidung wirken.

Neben angebots- oder nachfrageseitigen Erklärungsmodellen der Verrentungsentscheidung hat sich zudem in den 90er Jahren eine wissenschaftliche Diskussion über eine vermutete Tendenz hin zu geringerer Beschäftigungsstabilität und einem häufigeren Wechsel zwischen den Arbeitsplätzen entsponnen. Für ältere Beschäftigte würde ein solcher säkularer Trend besondere Risiken bergen, da ihre Wiedereinstellungschancen deutlich geringer sind als die jüngerer Kollegen. ${ }^{7}$ Allerdings sind die empirischen Befunde für Deutschland nicht eindeutig: Winkelmann und Zimmermann (1998) finden keine Anhaltspunkte für eine schwindende Stabilität von Beschäftigungsverhältnissen im Zeitraum von 1974 bis 1994. Anhand einer Auswertung des Sozio-Ökonomischen Panels (SOEP) mit Hilfe von Zähldatenmodellen sehen sie die landläufige Meinung nicht bestätigt, wonach sich die Beschäftigungssicherheit verringert habe. Allerdings berücksichtigen die Autoren nicht das Alter einer Person und lassen so keine Rückschlüsse auf das Beschäftigungswechselverhalten Älterer zu. Eine Studie von Bergemann und Mertens (2004) reicht bis in das Jahr 1999 und damit in den Beobachtungszeitraum

\footnotetext{
${ }^{7}$ So weisen Chan und Stevens (1999) anhand von Daten der Health and Retirement Study (HRS) nach, dass der Verlust des Arbeitsplatzes im Alter von 50 Jahren und älter zu einer gravierenden Verschlechterung der Arbeitsmarktaussichten dieser Personengruppe führt, die sich mit zunehmendem Alter weiter verschärft. Heywood et al. (1999) kommen für Hongkong zu demselben Ergebnis.
} 
dieser Arbeit. Ebenfalls anhand von SOEP-Daten kommen sie zu dem Schluss, dass sich die Medianverweildauer in einem Arbeitsverhältnis in den 1980er und 1990er Jahren verringert hat. Auch wenn die Befunde hinsichtlich der Beschäftigungsstabilität bislang nicht eindeutig sind, erscheint eine zeitlich flexible Schätzung der Verbleibewahrscheinlichkeit allein aus diesem Grunde notwendig. Zudem geben sie Anlass dazu, den gesamten Erwerbsverlauf der Personen mit zur Erklärung des Austrittsverhaltens hinzuzuziehen.

\section{A 1.1 Angebotsseitige Bestimmungsgründe}

Die meisten bisherigen Arbeiten zur Erwerbsbeteiligung im Alter und Wahl des Renteneintrittsalters untersuchen den Einfluss ausschließlich persönlicher Merkmale der Beschäftigten, die unter variierenden Nebenbedingungen über den Einsatz der Ihnen zur Verfügung stehenden Zeit entscheiden. Ausgangspunkt ist daher zumindest implizit immer ein mikroökonomisches Arbeitsangebotsmodell.

\section{A 1.1.1 Monetäre Einflussgrößen}

Darin machen die Arbeitnehmer ihre Entscheidung zur Weiter- bzw. Wiederbeschäftigung vor allem vom Vergleich des erzielbaren Arbeitseinkommens mit den Ersatzleistungen der Sozialversicherung abhängig. Großzügige Transferleistungen und geringe Zuwächse in den Anwartschaften bei späterer Inanspruchnahme verleiten die Wirtschaftssubjekte zu einem frühzeitigen Ausscheiden aus dem Arbeitsmarkt und lassen die Aufnahme einer neuen Beschäftigung unattraktiv erscheinen. So kann die Erwartung steigender Entlohnung, die sich aus einem höherem Alter oder einer längeren Betriebszugehörigkeit speist, ebenso einen verlängernden Effekt auf die Teilnahme am Erwerbsleben haben, wie Abschläge bei vorzeitigem Austritt. Unabhängig von der konkreten Ausgestaltung stellen Angebotsmodelle die Abwägung zwischen den beiden Möglichkeiten der Einkommenserzielung in den Mittelpunkt der Modellierung.

Arnds und Bonin (2003) unterscheiden dabei drei Gruppen von Ansatzpunkten: statische Modelle, dynamische Modelle und dynamische, strukturelle Modelle: Statische Modelle, wie sie in den ersten Studien (vgl. Feldstein (1974) und Boskin und Hurd (1978)) verwendet werden und in einer einfachen binären Regression der Verrentungsentscheidung münden, verwenden zur Erklärung der Entscheidung nur kontemporäre Variablen wie aktueller, entgangener Lohn und Rentenanwartschaften. Eine Erwartungsbildung über zukünftige Einkommens-, Konsumoder Gesundheitsgrößen wird in dieser Art von Modellen außen vor gelassen. Diesen Umstand griffen Kritiker auf, die den intertemporalen Charakter der Entscheidung deutlicher hervorzuheben suchten und den Optionswert der Verrentungsentscheidung mit ins Nutzenkalkül 
einfließen ließen. ${ }^{8}$ Die Entscheidung zwischen „Verbleib“ und „Austritt“ stellt sich hier als Abgleich des Barwertes der beiden Optionen dar. Eine weitere Neuerung brachten die strukturellen, dynamischen Modelle, die den Übergang in die Rente als sequenzielles Entscheidungsproblem auffassen und zu jeden (diskreten) möglichen Übergangszeitpunkt eine Nutzenabwägung modellieren, so dass ein ,optimal stopping problem' gelöst werden musste. ${ }^{9}$

\section{A 1.2.1.1. Lohn und Vermögen}

Eine zentrale Rolle bei der Abwägung zwischen Fortsetzung und Austritt aus der Erwerbstätigkeit spielt der Lohn als wesentlicher Bestandteil des Arbeitsentgeltes. Aus Sicht des Arbeitsanbieters stellen höhere Löhne höhere Opportunitätskosten der Verrentung dar und verzögern damit c.p. den Austritt aus dem Erwerbsleben. In den beschriebenen dynamischen Modellen der Renteneintrittsentscheidung geht der Lohn in den Optionswert der Schätzung ein.

Nach ökonomisch-theoretischen Modellen wirkt das Vermögen negativ auf das Arbeitsangebot Älterer und positiv auf die Übergangswahrscheinlichkeit in den Ruhestand. ${ }^{10}$ Auch in empirischen Untersuchungen ergeben sich für Vermögensvariable solche positiven Einflüsse. Für Deutschland weisen dies z.B. Börsch-Supan und Berkel (2004) nach, für die Niederlande kommt zuletzt Bloemen (2008) zu demselben Ergebnis. ${ }^{11}$

Für Deutschland zeigen Böhm und Merz (2008) anhand von Steuerdaten eine mit steigendem Alter ebenfalls ansteigende Konzentration der Einkommen, die die Relevanz für die Erwerbsentscheidung der hier relevanten Kohorten unterstreicht.

\section{A 1.2.1.2 Transferleistungen}

Die bei einem Austritt zu erwartenden Transferleistungen stehen dem erzielbaren Lohn als Parasympathikus entgegen. Sie sind den Regelungen des Rentenrechtes (SGB VI) und des

\footnotetext{
${ }^{8}$ Beispiele für eine solche theoretische Behandlung des Themas für das deutsche Alterssicherungssystem sind die Arbeiten von Börsch-Supan (1992), Schmidt (1995), Riphahn und Schmidt (1997) sowie Viehbrok (1997 und 1998).

${ }^{9}$ Meist besteht der Ausgangspunkt der Analysen in der Formulierung der Entscheidung als intertemporales Problem, dessen Lösung nicht nur die Erwerbsentscheidung endgültig löst, sondern auch das verbleibende Lebenseinkommen determiniert. Technisch betrachtet handelt es sich dabei um eine dynamische diskrete Entscheidungssituation, in der nach jedem Zeitintervall eine erneute Wahl getroffen werden muss, solange der als unumkehrbar angenommene Entschluss zum Eintritt in die Rente nicht gefasst wurde. Allerdings ließen sich solche Modelle aufgrund der rechenintensiven Anforderungen erst mit dem Aufkommen von leistungsstarken Computern schätzen. Für Beispiele solcher Modelle vgl. Gustman und Steinmeier (1986), Rust (1989), Rust und Phelan (1997), Blau und Gilleskie (1998), Heyma (2001) und Heyma (2004). Knaus (2003) liefert eine Schätzung für Deutschland.

${ }^{10}$ Vgl. z.B. Blundell et al. (1997).

${ }^{11}$ Im Speziellen betrachten Coile und Levine (2004) den Einflusses des Buchwertes von Aktien auf die Verrentungsentscheidung für die USA. Ausgehend von Daten aus den Boomzeiten der Jahrtausendwende kommen sie allerdings zu dem Schluss, dass dieser Höhenflug nur eine geringe Anzahl von Personen zu einer Frühverrentung bewogen hat.
} 
Arbeitsförderungsgesetzes (SGB III, vor 1998 AFG) zu entnehmen, die die verschiedenen Möglichkeiten und Bedingungen des Übergangs aus dem Erwerbsleben festlegen. Diese Bedingungen für den Rentenzugang haben sich in den vergangenen Jahren, und damit im Beobachtungszeitraum dieser Untersuchung, erheblich geändert. Die Entwicklung der Rentenpolitik im Beobachtungszeitraum und damit auch die Möglichkeiten zum finanziell durch die gesetzliche Rente gesicherten Abschied aus dem Erwerbsleben werden im Folgenden dargelegt. ${ }^{12}$

Spätestens in den 1990er Jahren hat sich ein Kurswechsel in der Rentenpolitik vollzogen: weg von einer den Arbeitsmarkt entlastenden Anreizsetzung zur Frühverrentung hin zu einer verstärkten Erwerbsbeteiligung Älterer. ${ }^{13}$ Die schnelle Abfolge von Rechtsänderungen spiegelt auch die intensive Diskussion um die Zukunft und Finanzierung der gesetzlichen Rente: Das Rentenreformgesetz 1992 sah u. a. die stufenweise Heraufsetzung der vorzeitigen und flexiblen Altersgrenzen von 60 bzw. 63 Jahren auf 65 Jahre vor. Anfang 1997 trat das Wachstumsund Beschäftigungsförderungsgesetz in Kraft. Darin war u. a. eine vorgezogene Umsetzung der Anhebungen der Altersgrenzen enthalten, wie sie die Rentenreform 1992 vorgesehen hatte. Eine weitere Rentenreform 1999 erschwerte den vorzeitigen Eintritt in die staatliche Rente erneut: Mit der Anhebung der Altersgrenzen für Schwerbehinderte und der gleitenden Abschaffung der Altersrente wegen Arbeitslosigkeit und Altersteilzeit sowie der vorzeitigen Altersrente für Frauen wurden dem frühen Ausstieg aus dem Erwerbsleben weitere Hürden auferlegt. Zwar wurde 1998 die Anhebung der Grenze für Schwerbehinderte wieder bis Ende 2000 ausgesetzt, aber die Erwerbsminderungsrenten-Reform, die 2001 in Kraft trat, löste die Berufsunfähigkeitsrente aus dem Leistungskatalog der gesetzlichen Sozialversicherung heraus und hob die Altersgrenze für Schwerbehinderte auf 63 Jahre an.

Welche Rentenzugangswege standen älteren Arbeitnehmern in Deutschland im Untersuchungszeitraum offen? Zunächst unterscheidet man verschiedene Rentenarten. Neben die Altersrente treten die Berufs- und Erwerbsunfähigkeitsrente und die Renten wegen Todes. Nur

\footnotetext{
${ }^{12}$ Ausführliche Überblicke über die Eintrittspfade in die gesetzliche Rente geben u.a. auch Rosenow und Wagner (1994), Gatter und Hartmann (1995), Brühl (1997) und Michaelis und Rieckhoff (2003).

Eine frühe Abschätzung möglicher Politikfolgen anhand eines Mikrosimulationsmodells bieten Schatz und Merz (2000), die Erwerbsbiografien nach Änderungen des Zugangsrechts und dadurch ausgelösten Erwerbsverhaltensänderungen fortschreiben. Sie stützen sich dabei auf eine Stichprobe aus den Versichertenkonten der Gesetzlichen Rentenversicherung, die sie mit eigens erhobenen Befragungsdaten kombinieren. Vgl. auch Merz et al. (2004).

${ }^{13}$ Schon vor der Rentenreform 1973 gab es in Deutschland zahlreiche Möglichkeiten des Rentenzugangs vor dem gesetzlich festgelegten Renteneintrittsalter. So hatten Arbeitslose seit der Rentenreform 1957 die Möglichkeit, mit 60 Jahren ihre Altersrente anzutreten. Die 2. Rentenreform von 1973 flexibilisierte die bis dahin noch starreren Altersgrenzen mittels eines vorgezogenen Altersruhegeldes für langjährig Versicherte und führte das hinausgeschobene Altersruhegeld und Altersrenten für Schwerbehinderte sowie Berufs- und Erwerbsunfähige neu ein. Das vorgezogene Ruhestandsalter für Schwerbehinderte wurde 1979 von 62 auf 61 Jahre, ab 1980 auf 60 Jahre abgesenkt.
} 
der Zugang zu den beiden erstgenannten soll hier dargestellt werden, da nur für diese ein erkennbarer Zusammenhang zwischen betrieblichem Verhalten und Renteneintritt besteht.

\section{A 1.2.1.2.1 Altersrente}

Bestand die Zielsetzung der Altersrente historisch zunächst darin, Ruheständler in den letzten Jahren ihres Lebens vor Armut zu schützen, erfüllt sie heute die Aufgabe, den Lebensstandard aus dem Erwerbsleben auch im Ruhestand zu erhalten. Zudem sind im deutschen Rentensystem seit vielen Jahren implizit auch andere Lebensrisiken mitversichert, die typischerweise am Ende des Erwerbslebens mit höherer Wahrscheinlichkeit auftreten wie Erwerbsunfähigkeit und Arbeitslosigkeit. Dies drückt sich in Möglichkeiten des vorgezogenen Zugangs in die Altersrente aus, die Gegenstand der folgenden Seiten sind.

Den zumindest offiziell gängigen Weg in die Rente stellt die Regelaltersrente dar, die mit 65 Jahren ohne Abzüge ${ }^{14}$ angetreten werden kann. Anders als in vielen anderen EU-Staaten sieht die Altersrente kein obligatorisches Renteneintrittsalter vor. ${ }^{15}$ Die allgemeine Wartezeit beträgt fünf Jahre. ${ }^{16}$ Eine Hinzuverdienstgrenze gilt für die Altersgrenze nicht.

Neben der Regelaltersrente standen zum Zeitpunkt der Untersuchung einige andere Zugangsarten zur Altersrente offen. ${ }^{17}$ Da diese einen im Sinne der Regelaltersrente vorzeitigen Eintritt

\footnotetext{
${ }^{14}$ Die monatliche Bruttorente ergibt sich aus der Multiplikation von persönlichen Entgeltpunkten und aktuellem Rentenwert. Die Entgeltpunkte berechnen sich zunächst aus dem Verhältnis des Einkommens des Versicherten mit dem Durchschnittseinkommen aller Versicherten des jeweiligen Kalenderjahres. Die Entgeltpunkte der einzelnen Jahre werden addiert. Danach wird diese Summe dann mit dem Zugangsfaktor multipliziert, der eine Anpassung der Rentenhöhe an den gewählten Renteneingangszeitpunkt darstellt. Der Zugangsfaktor beträgt zunächst 1,0. Er steigt, wenn eine Rente wegen Alters trotz erfüllter Wartezeit erst nach Vollendung des 65. Lebensjahres in Anspruch genommen wird. Abschläge sind hinzunehmen, wenn eine Rente vorzeitig in Anspruch genommen wird. Nach dieser Multiplikation erhält man die persönlichen Entgeltpunkte. Die Bruttorente ergibt sich dann nach Multiplikation mit dem Rentenwert. Dies ist der Betrag, der einer monatlichen Rente aus Beiträgen eines Durchschnittverdieners für ein Jahr entspricht.

${ }^{15}$ Auch ein Erreichen der Altersgrenze für die abschlagsfreie Altersrente berechtigt den Arbeitgeber nach dem KSchG nicht zu einer Kündigung. Sie ist nur dann rechtens, wenn dem Beschäftigten nachgewiesen werden kann, dass er seinen Aufgaben nicht länger gewachsen ist. Allerdings enthalten nicht nur individuelle Verträge i.d.R. Klauseln, die ein Ende des Arbeitsverhältnisses an ein festgelegtes Alter knüpfen, sondern auch viele Tarifverträge und Betriebsvereinbarungen (vgl. Schmidt (2003), S. 361).
}

${ }^{16}$ Für viele Rentenzugangsarten gilt, dass vor Rentenantritt eine Wartezeit - Mindestversicherungszeit - verstrichen sein muss, ab der man rentenberechtigt ist. Je nach Rentenart werden der Wartezeit über die Versicherungszeit hinaus weitere Zeiten angerechnet. Für Wartezeiten von fünf, fünfzehn und zwanzig Jahren werden neben Beitrags- und Ersatzzeiten auch Zeiten aus dem Versorgungsausgleich und aus geringfügiger Beschäftigung berücksichtigt. Unter Ersatzzeiten versteht man Dies sind Zeiten ohne Beitragsleistung, weil der Versicherte - aus Gründen, die nicht in seiner Person lagen - an der Zahlung von Beiträgen gehindert war, z. B. durch Kriegsgefangenschaft, NS-Verfolgung, Flucht und politische Haft in der DDR. Zu den Beitragszeiten zählen auch Zeiten der Kindererziehung: Für jedes vor 1992 geborene Kind wird dem erziehenden Elternteil eine Beitragszeit von einem Jahr unterstellt, wobei ein durchschnittlicher Verdienst unterstellt wird. Für jedes später geborene Kind werden drei Jahre auf die Beitragszeit angerechnet. Für einzelne Rentenzugangsarten kommen Anrechnungs-, Berücksichtigungs- und Zurechnungszeiten, somit alle rentenrechtlichen Zeiten, hinzu (vgl. Bundesministerium für Gesundheit und Soziale Sicherheit (2005)).

${ }^{17}$ Die Möglichkeit, schon vor der eigentlichen Altersgrenze in den Ruhestand zu gehen, besteht schon lange: Im März 1929 wurde für arbeitslose Angestellte, die das 60. Lebensjahr vollendet hatten und ohne Anspruch auf Arbeitslosenunterstützung blieben, die Möglichkeit eröffnet, die Altersrente schon vorzeitig zu beziehen. Für 
in die Rente darstellen, sind sie hier von besonderer Wichtigkeit. Die Zugangspfade sind im Einzelnen:

- $\quad$ die Altersrente wegen Arbeitslosigkeit,
-
-
-
die Altersrente bei verminderter Erwerbsfähigkeit,
die Altersrente nach Altersteilzeit und
die vorgezogene Altersrente für Frauen.

Bis Ende 1991 musste man bei vorzeitigem Renteneintritt keinen Abschlag hinnehmen. Mit den Rentenreformen der Jahre 1992, 1999 und 2001 sind die Möglichkeiten für den vorzeitigen Renteneintritt stark eingeschränkt worden. Die Änderungen werden mitsamt den Rentenzugangsarten im Folgenden beschrieben. Eine Zusammenfassung der Zugangsarten findet sich in Tab. 2. Bezieher einer vorgezogenen Altersrente dürfen maximal $345 €$ brutto hinzuverdienen. $^{18}$

Altersrente wegen Arbeitslosigkeit: Eine besondere Art des Rentenzugangs wird Arbeitslosen gewährt. Sie können mit 60 Jahren aus dem Erwerbsleben treten, solange sie bei Antritt ihrer Rente arbeitslos waren und nach dem Alter von 58,5 Jahren ein Jahr lang arbeitslos waren. Zudem müssen sie innerhalb der 10 Jahre vor Rentenbeginn mindestens 8 Jahre mit Pflichtbeitragszeiten und eine Wartezeit von mindestens 15 Jahren vorweisen. ${ }^{19}$

Im Verlauf der 90er Jahre wurde der Zugang zu dieser Rentenart erschwert und läuft in den kommenden Jahren vollständig aus. Von 1997 an wurde die Altersgrenze, also das Alter, ab dem ein abschlagfreier Eintritt möglich ist, stufenweise von 60 auf 65 Jahre angehoben. Auch die Altersgrenze für eine vorzeitige Inanspruchnahme der Versicherungsleistung wurde auf 63 Jahre heraufgesetzt. Danach konnte, wer zwischen 1937 und 1945 geboren ist und die weiteren Anforderungen erfüllt, zwar weiterhin mit Vollendung des 60. Lebensjahres in Rente gehen, aber es ergeben sich je nach Alter und Renteneintrittsalter Abschläge von bis zu 18

\footnotetext{
Arbeiter wurde diese Regelung im Jahr 1957 ausgedehnt. Ebenfalls seit 1957 konnten Frauen bis zu 5 Jahre vor der regulären Altersgrenze von 65 in den bezahlten Ruhestand gehen. Die erste große Rentenreform im Jahr 1973 führte dann u.a. die flexible Altersgrenze für Versicherte mit mindestens 35 Versicherungsjahren ein. Diese Personengruppe konnte sich ab einem Alter von 63 Jahren verrenten lassen. Schwerbehinderte, Berufs- oder Erwerbsunfähige konnten ab 1972 mit 62 in Rente gehen, später gar ab einem Alter von 60 Jahren.

${ }^{18}$ Stand: Dezember 2005; Innerhalb eines Kalenderjahres ist ein zweimaliges Überschreiten dieses Betrages bis zum Doppelten (zum Beispiel durch Urlaubs- und Weihnachtsgeld) zulässig (vgl. Bundesministerium für Gesundheit und Soziale Sicherung (2005), S. 95). Für Bezieher einer Teilrente gelten höhere Hinzuverdienstgrenzen.

${ }^{19}$ Die Regelung wurde im Jahr 1957 eingeführt, aber erst ab den 1970er Jahren in Zeiten höherer Arbeitslosigkeit in nennenswertem Umfang genutzt. Diese Form des Ausscheidens aus dem Erwerbsleben - umgangssprachlich auch als 59er-Regelung bekannt - etablierte sich als indirekter Rentenzugang, der über den Umweg der Arbeitslosigkeit schon vor dem 60. Lebensjahr den Ausstieg aus dem Erwerbsleben zulies (vgl. Gatter und Hartmann (1995), S. 414). Dabei wurden Leistungen der Arbeitslosenversicherung in Höhe von 60 bzw. 67 \% (bei Arbeitslosen mit Kindern) des letzten Nettoverdienstes vom ehemaligen Arbeitgeber aufgestockt, bis die Altersgrenze von 60 Jahren erreicht war.
} 
Tab. 2: Zugangsarten zur Altersrente: Regelungen zu Beginn und Änderungen im Beobachtungszeitraum

\begin{tabular}{|c|c|c|c|c|c|}
\hline $\begin{array}{l}\text { ALTERSRENTEN- } \\
\text { ZUGANG }\end{array}$ & $\begin{array}{l}\text { FRÜHESTER } \\
\text { EINTRITT } \\
\end{array}$ & $\begin{array}{l}\text { WAR- } \\
\text { TEZEIT }\end{array}$ & $\begin{array}{l}\text { WEITERE } \\
\text { VORAUSSETZUNGEN }\end{array}$ & $\begin{array}{l}\text { HINZUVER- } \\
\text { DIENST } \\
\end{array}$ & $\begin{array}{l}\text { ÄNDERUNGEN } 1996 \\
\text { BIS } 2004\end{array}$ \\
\hline Regelaltersrente & 65 Jahre & 5 Jahre & & Unbegrenzt & \\
\hline $\begin{array}{l}\text { Altersrente wegen Ar- } \\
\text { beitslosigkeit }\end{array}$ & 60 Jahre & 15 Jahre & $\begin{array}{l}\text { Bei Eintritt und mindestens } \\
12 \text { von } 18 \text { Monaten vor } \\
\text { Eintritt arbeitslos, } 8 \text { Jahre } \\
\text { Pflichtbeiträge von zehn } \\
\text { Jahren vor Eintritt }\end{array}$ & $\begin{array}{l}\text { Je nach Teilrente } \\
\text { und dem zuletzt ver- } \\
\text { sicherten Verdienst; } \\
\text { getrennt nach Ost } \\
\text { und West }\end{array}$ & $\begin{array}{l}\text { Die Altersgrenze wurde } \\
\text { von } 1997 \text { an auf } 65 \text { Jahre } \\
\text { angehoben. Früherer Ein- } \\
\text { tritt mit Abschlägen mög- } \\
\text { lich. }\end{array}$ \\
\hline $\begin{array}{l}\text { Altersrente für Schwerbe- } \\
\text { hinderte, Berufs- und Er- } \\
\text { werbsunfähige }\end{array}$ & 60 Jahre & 35 Jahre & $\begin{array}{l}\text { Bei Beginn der Rente } \\
\text { schwer behindert (Grad der } \\
\text { Behinderung mindestens } \\
50 \text { Prozent), berufs- oder } \\
\text { erwerbsunfähig }\end{array}$ & $\begin{array}{l}\text { Je nach Teilrente } \\
\text { und dem zuletzt ver- } \\
\text { sicherten Verdienst; } \\
\text { getrennt nach Ost } \\
\text { und West }\end{array}$ & $\begin{array}{l}\text { Die Altersgrenze wurde } \\
\text { von } 2001 \text { an auf } 63 \text { Jahre } \\
\text { angehoben. Früherer Ein- } \\
\text { tritt mit Abschlägen mög- } \\
\text { lich. }\end{array}$ \\
\hline $\begin{array}{l}\text { Altersrente für langjährig } \\
\text { Versicherte }\end{array}$ & 62 Jahre & 35 Jahre & & $\begin{array}{l}\text { Je nach Teilrente } \\
\text { und dem zuletzt ver- } \\
\text { sicherten Verdienst; } \\
\text { getrennt nach Ost } \\
\text { und West }\end{array}$ & $\begin{array}{l}\text { Die Altersgrenze wurde } \\
\text { von } 2000 \text { an auf } 65 \text { Jahre } \\
\text { angehoben. Früherer Ein- } \\
\text { tritt mit Abschlägen mög- } \\
\text { lich. }\end{array}$ \\
\hline $\begin{array}{l}\text { Altersrente nach Alters- } \\
\text { teilzeit }\end{array}$ & 60 Jahre & 15 Jahre & $\begin{array}{l}\text { Mind. } 24 \text { Monate Alters- } \\
\text { teilzeitarbeit, innerhalb der } \\
\text { letzten } 10 \text { Jahre vor Eintritt } \\
\text { mind. } 8 \text { Jahre mit Beitrags- } \\
\text { zeiten }\end{array}$ & $\begin{array}{l}\text { Je nach Teilrente } \\
\text { und dem zuletzt ver- } \\
\text { sicherten Verdienst; } \\
\text { getrennt nach Ost } \\
\text { und West }\end{array}$ & $\begin{array}{l}\text { Die Altersgrenze wurde } \\
\text { von } 1997 \text { an auf } 65 \text { Jahre } \\
\text { angehoben. Früherer Ein- } \\
\text { tritt mit Abschlägen mög- } \\
\text { lich. }\end{array}$ \\
\hline $\begin{array}{l}\text { Vorgezogene Altersrente } \\
\text { für Frauen }\end{array}$ & $\begin{array}{l}60 \text { Jahre } \\
\text { (bzw. } 65 \text { Jahre) }\end{array}$ & 15 Jahre & $\begin{array}{l}\text { nach Vollendung des } 40 \text {. } \\
\text { Lebensjahres mehr als } 10 \\
\text { Jahre Pflichtbeitragszeit }\end{array}$ & & $\begin{array}{l}\text { Die Altersgrenze wurde } \\
2000 \text { für ab } 1940 \text { Gebore- } \\
\text { ne auf } 65 \text { Jahre angeho- } \\
\text { ben. Früherer Eintritt mit } \\
\text { Abschlägen möglich. }\end{array}$ \\
\hline
\end{tabular}

Quelle: Bundesversicherungsanstalt für Angestellte, VDR(1997 und 1999), eigene Erstellung 
Prozent (pro Monat vorzeitigem Eintritt gehen 0,3 Prozent der Rentenansprüche dauerhaft verloren). ${ }^{20}$ Damit betrifft diese Regeländerung genau die untersuchte Personengruppe.In den Jahren 1996 bis 2003 sank der Anteil unter den jeweils 60-Jährigen an der Gesamtbevölkerung, die eine vorgezogene Altersrente wegen Arbeitslosigkeit beantragten, von rd. 17 Prozent auf ungefähr 5 Prozent (vgl. Büttner (2005), S. 6). Zwar stiegen im zeitlichen Verlauf die Zugangsanteile für Personen zwischen 61 und 64 Jahren leicht an, doch insgesamt sank die Bedeutung dieser Rentenart im Beobachtungszeitraum ab. Zwei Gründe sprechen nach Büttner (2005, S. 7) für einen Rückgang in der Wahl dieses Rentenzugangs zum frühest möglichen Zeitpunkt: Zum einen greift die Einführung von Rentenabschlägen, zum anderen zeigt das Ende des Altersübergangsgeldes in der ehemaligen DDR Ende 1992 seine Wirkung, das spätestens Ende 1997 zu einer Rente wegen Arbeitslosigkeit im Alter von 60 Jahren führte.

Altersrente für langjährig Versicherte: Im Jahr 1996 konnten Versicherte mit einer Wartezeit von mindestens 35 Jahren ab dem 63. Geburtstag ohne Abzüge ihre Rentenansprüche geltend machen. Mit Wirkung zum 1.1.1999 wurde die Altersgrenze schrittweise von 63 auf 65 Jahre angehoben. So konnten die betroffenen Jahrgänge von da an nur noch mit Abschlägen in Rente gehen. Für 1939 und später Geborene gilt inzwischen eine Altersgrenze von 65 Jahren. Durch die beschlossene Absenkung des Mindestalters von 63 auf 62 Jahre wird diese Rentenart langfristig abgeschafft, da sich die Zugangsregeln nicht von denen der Regelaltersrente unterscheiden.

Im Zeitraum von 1996 bis 2003 lässt sich der erstmalige Abschlag im Jahr 2000 deutlich am Anteil der 63-Jährigen erkennen, die eine solche Rentenzugangsart wählen. 1997, 1998 und 1999 steigt der Anteil an, um danach deutlich abzufallen (vgl. Büttner (2005)).

Altersrente für Schwerbehinderte: Auf die Altersrente für Schwerbehinderte hat Anspruch, wer das 63. Lebensjahr vollendet hat, bei Beginn der Altersrente anerkannter Schwerbehinderter mit einem Behinderungsgrad von mindestens 50 Prozent und die Wartezeit von 35 Jahren erfüllt hat. Mit den üblichen Abschlägen von 0,3 Prozent pro Monat kann man die Altersrente für Schwerbehinderte schon im Alter von mindestens 60 Jahren vorzeitig antreten.

Die Altersgrenze wurde für diese Rentenart ab 2001 schrittweise von 60 auf 63 Jahre angehoben. Von der Anhebung betroffen waren nach 1940 geborene Versicherte. Eine vorzeitige Inanspruchnahme dieser Altersrente ist frühestens ab Vollendung des 60. Lebensjahres mög-

\footnotetext{
${ }^{20}$ Ein Vertrauensschutz wurde bei der Anhebung im Jahr 1997 für Versicherte eingerichtet, die vor dem 1.1.1942 geboren wurden, und mindestens 45 Jahre Pflichtbeiträge bezahlt haben, für solche, die zum Stichtag 14.2.1996 arbeitslos und nicht älter als 55 Jahre waren, sowie für Beschäftigte der Montanindustrie. Für vor 1941 Geborene dieser Gruppen war ein vorzeitiger Renteneintritt mit 60 Jahren weiterhin möglich. Für die Jüngeren erfolgte die Anpassung der Altersgrenzen in Viermonatsschritten.
} 
lich. ${ }^{21}$ Bis Ende 2000 konnte diese Rente auch in Anspruch genommen werden, wenn statt einer Schwerbehinderung Berufs- oder Erwerbsunfähigkeit vorlag.

Die Altersrente für Schwerbehinderte hat in den vergangenen Jahren stetig zugenommen. Während 1996 in Deutschland noch gut 45.000 Menschen diese Form der Altersrente erstmals bezogen, lag die Zahl zehn Jahre später bei knapp 73.000. Der Anteil unter den Rentenzugängen aller Versichertenrenten stieg derweil von 4,3 Prozent auf 7,3 Prozent. Seit 2001 gelten Abschläge für schwer behinderte 60-Jährige. Trotzdem stieg bis 2002 der Anteil in dieser Kohorte. Büttner (2005, S. 9) macht für diese Beobachtung mögliche Ausweicheffekte aus anderen Zugangsformen und umfangreiche Vertrauensschutzregelungen verantwortlich.

Altersrente nach Altersteilzeit: Eine Form des kontinuierlichen Übergangs aus dem Erwerbsleben stellt die Altersteilzeit dar. Danach kann ein Versicherter seine Arbeitszeit auf die Hälfte reduzieren und bis zum Bezug der Altersrente Zuschüsse von der Bundesagentur für Arbeit (BA) beziehen: Gemäß Altersteilzeitgesetz (ATG) von $1996^{22}$ können ältere Arbeitnehmer ihre Arbeitszeit mit Zuschüssen auf die Hälfte zu verringern, wenn sie das 55. Lebensjahr vollendet haben und innerhalb der fünf Jahre vor Beginn der Altersteilzeit mindestens drei Jahre sozialversicherungspflichtig beschäftigt gewesen sind. Die Vereinbarung muss dabei immer mindestens bis zur Rente reichen. Altersteilzeitarbeit wird von der BA nur dann gefördert, wenn der Arbeitgeber die frei werdende Stelle durch einen Arbeitslosen oder die Weiterbeschäftigung eines Auszubildenden wieder besetzt. ${ }^{23}$

\footnotetext{
${ }^{21}$ Versicherte, die zu den so genannten rentennahen Jahrgängen gehören, können sich wieder auf einen Vertrauensschutz bei der Anhebung der Altersgrenze berufen, wenn sie vor dem 1. Januar 1942 geboren sind und mindestens 45 Jahre mit Pflichtbeiträgen für eine versicherte Beschäftigung aufweisen können oder bis zum 16.11.1950 geboren sind und am 16.11.2000 schwer behindert, berufsunfähig oder erwerbsunfähig nach dem am 31.12.2000 geltenden Recht waren. Der Vertrauensschutz bewirkt, dass eine Anhebung der Altersgrenze nicht erfolgt - die Rente kann also mit 60 Jahren ungekürzt in Anspruch genommen werden.

${ }^{22}$ Vorgänger dieser Regelung war das von 1984 an gültige Vorruhestandsgesetz. Nach der bis 1988 befristeten Regelung hatten Beschäftigte das Recht, nach Vollendung des 58. Lebensjahres unter Fortzahlung von reduzierten Bruttogehältern bis zum Eintritt in die Altersrente durch den Arbeitgeber frühzeitig gänzlich aus dem Erwerbsleben auszuscheiden. Auch hier wurden Zuschüsse gewährt, wenn der Arbeitgeber Lehrlinge oder Arbeitslose auf der frei gewordene Stelle einsetzte. Dieser für die Arbeitgeber teure Renteneintritt wurde 1989 durch den Rechtsanspruch auf Altersteilzeit ersetzt, der ältere Arbeitnehmer stärker an den Kosten des vorgezogenen Ausscheidens aus der Beschäftigung beteiligte und zugleich die bei den Unternehmen anfallenden Kosten durch vermehrte Zuschüsse durch die BA senkte (vgl. Arnds und Bonin (2003)). Die Regelungen dieses Altersteilzeitgesetzes, das in den Jahren 1989 bis 1992 bestand, wurde allerdings nur in sehr geringem Maße in Anspruch genommen (vgl. Menges (2001)). Zudem bestand in den Ostdeutschland nach der Vereinigung der beiden Staaten für Arbeitslose ab dem Alter von 57 Jahren (ab 199155 Jahren) die Möglichkeit, ein „Altersübergangsgeld“ (§ 249c AFG, vgl. Hoffmann (1996)) in Höhe von 65\% des letzten durchschnittlichen Nettolohnes zu beziehen bis zum Eintritt in die Altersrente (vgl. Kirner und Meinhardt (1997) und Autorengemeinschaft (1991)). Hintergrund für die Einführung des Gesetzes 1996 war der starke Zuwachs an Frühverrentungen zu Beginn der 90er Jahre. Dies verdeutlicht z.B. die Entwicklung der Renten wegen Arbeitslosigkeit, die zwischen 1992 und 1995 von 7,3\% auf $24 \%$ der Neurenten anstiegen. Arbeitsmarktpolitisches Ziel der Einführung war zum einen, Ältere vor der Arbeitslosigkeit zu bewahren und gleichzeitig Jüngeren den Zugang in den Arbeitsmarkt zu erleichtern.

${ }^{23}$ In Kleinunternehmen mit nicht mehr als 50 Arbeitnehmern wird die Altersteilzeit auch dann gefördert, wenn aus Anlass der Altersteilzeit ein Auszubildender eingestellt wird (vgl. BMWA (2004), S. 8).
} 
Die BA erstattet zu sechs Jahre Zuschläge ${ }^{24}$ auf das halbe Arbeitsentgelt, so dass dem Arbeitnehmer mindestens 70 Prozent seines früheren Nettoentgelts verbleiben. Um den Ausfall von Ansprüchen auf Altersrente auszugleichen, stockt die BA zudem die Rentenversicherungsbeträge des Teilzeitbeschäftigten auf, so dass diese mindestens 90 Prozent der bisherigen betragen. Soweit die Tarifvertragsparteien es beschließen, kann die Altersteilzeit auf maximal zehn Jahre verlängert werden (vgl. Engstler (2004), S. 26). Dabei kann man zwischen einer echten Teilzeitvereinbarung und einem Blockmodell wählen, ${ }^{25}$ Im Gegensatz zu anderen Formen des Übergangs in die Rente müssen für die Altersteilzeit sowohl Arbeitnehmer als auch -geber zustimmen. Nachdem die Altersteilzeit bis zum Jahr 2001 in über 400 Tarifverträgen vereinbart worden war und damit rd. 13 Mio. Beschäftigte abgedeckt waren (vgl. BMFSFJ (2001), S. 32), galten im Juni 2004 schon 872 Tarifverträge mit Regelungen der Altersteilzeit (vgl. BMWA (2004), S. 55-93).

Im Anschluss an die Altersteilzeit bestand zu Beginn der Beobachtungszeit die Möglichkeit, im Alter von 60 Jahren ohne Abschläge in Rente zu gehen, wenn die Altersteilzeit mindestens zwei Jahre angedauert hat, mindestens acht der zehn Jahre vor Rentenbeginn Beiträge gezahlt wurden und eine Wartezeit von 15 Jahren erreicht wurde. Für die Jahrgänge ab 1937 wurden ab 1997 ebenso wie bei der Altersrente wegen Arbeitslosigkeit Anpassungen hin zu einem Eintrittsalter von 65 eingeführt, die zu Abschlägen in der Rentenhöhe je nach Geburtsmonat führen.

Die Rente nach Altersteilzeit tritt erst vier Jahre nach Einführung, nämlich im Jahr 2000 nennenswert in Erscheinung. Bis 2002 steigt ihr Anteil in allen Altersklassen. Ein Grund könnte darin liegen, dass Arbeitgeber die abschlagbedingten Rentensenkungen finanziell ausgleichen (vgl. Stück (2003) ff.), da für die jeweils jüngeren Kohorten bereits Abschlagsregelungen gelten.

Vorgezogene Altersrente für Frauen: Einen besonderen Zugangsweg sieht das Rentenrecht für Frauen vor: Die vorgezogene Altersrente für Frauen nimmt dabei Rücksicht auf frauentypische Erwerbsbiographien, deren Beitragszahlungen von Erziehungspause unterbrochen sind. Für langjährig versicherte Beitragszahlerinnen bestand schon seit 1957 die Möglichkeit zum Eintritt in die Altersrente, sofern sie eine Beitragszeit von mindestens 15 Jahren vorweisen konnten. Davon mussten mindestens zehn nach dem vierzigsten Lebensjahr erbracht sein. Auch diese vorgezogene Altersrente steht vor dem Aus. Seit dem Jahr 2000 wurde hier die Altersgrenze ebenfalls schrittweise auf 65 Jahre angehoben.

\footnotetext{
${ }^{24}$ Bis zu einer Änderung im Juli 2000 durch das „Zweite Gesetz zur Fortentwicklung der Altersteilzeit“ betrug die Förderungshöchstdauer lediglich fünf Jahre.

${ }^{25}$ Diese zweite Variante wird von der Mehrzahl der Betriebe angewandt. So wurden beispielsweise in den Monaten Januar bis September 2001 knapp 87\% der Neuzugänge in die ATG als Blockmodell beantragt (vgl. Achternhagen (2001), S. 125).
} 
Der Anteil der 60-Jährigen an der Gesamtbevölkerung, die eine vorgezogene Altersrente für Frauen beantragten, ist zwischen 1996 und 2003 stark zurückgegangen. Bis 1999 lässt sich noch ein Anstieg verzeichnen, den Büttner (2005, S. 8) auf eine Ausweichreaktion auf den bereits 1997 einsetzenden Abschlag auf Renten wegen Arbeitslosigkeit zurückführt. Nach 1999 sinkt der Anteil von knapp 20 Prozent auf 11,5 Prozent im Jahr 2003. Vieles spricht bei dieser Rentenzugangsart für eine Verzögerung des Eintritts, da gleichzeitig mit dem Rückgang des Anteiles unter den 60-Jährigen derjenige für die jeweils älteren Kohorten in den Jahren 1999 bis 2003 zum Teil stark ansteigt.

\section{A 1.2.1.2.2 Berufs- und Erwerbsunfähigkeit}

Eine weitere Form des frühzeitigen Austritts aus dem Erwerbsleben stellt der Wechsel in die Erwerbs- und Berufsunfähigkeitsrente (EU- bzw. BU-Rente) dar. Seit Anfang 2001 ist diese Rentenart von einer teilweisen oder vollständigen Erwerbsminderungsrente abgelöst.

Bis zu diesem Zeitpunkt sah die die Regelung vor, dass Anspruch auf eine Erwerbs- oder Berufsunfähigkeitsrente hatte, wer erwerbs- bzw. berufsunfähig war, in den letzten fünf Jahren vor Eintritt der Berufsunfähigkeit drei Jahre Pflichtbeitragszeiten und die allgemeine Wartezeit von fünf Jahren erfüllt hatte.

Erwerbsunfähig war demnach, wer aus gesundheitlichen Gründen eine regelmäßige Erwerbstätigkeit nicht mehr ausüben oder nur ein geringfügiges Einkommen erzielen konnte. Berufsunfähig war, wer aus gesundheitlichen Gründen in seinem oder einem anderen zumutbaren Beruf weniger als die Hälfte leisten konnte wie gesunde Berufstätige. Auf das Lebensalter kam es in beiden Rentenfällen nicht an. Für BU-Rentenbezieher mit einem Rentenbeginn vor 1996 galt bis Ende 2000 keine Hinzuverdienstbeschränkung. Seit 2001 müssen alle Beziehern einer Rente wegen Erwerbsminderung Hinzuverdienstgrenzen zu beachten.

Bei der Gesetzesänderung zum Jahr 2001 wurde ein einheitliches abgestuftes System der Erwerbsminderungsrente eingeführt, das volle Erwerbsminderung für Versicherte vorsieht, sobald diese weniger als drei Stunden täglich arbeiten können. Eine teilweise Erwerbsminderung liegt vor, wenn die Person drei bis sechs Stunden täglicher Arbeit verrichten kann.

Zentrales Element der Neuregelung war allerdings die Abschaffung der Berufsunfähigkeitsrente. Mit dem Wegfall des Berufsschutzes wurden subjektive Zumutbarkeit und eine der Ausbildung angemessene Beschäftigung als Kriterien für die Aufnahme in die Rente ausgeschlossen. Stattdessen ist die Leistungsfähigkeit des Versicherten auf dem allgemeinen Arbeitsmarkt maßgeblich für einen Rentenanspruch.

Die vorgestellten Änderungen schlagen sich nieder in Änderungen im Zugangsverhalten in den Ruhestand. Tab. 3 stellt den Verlauf der Zugänge in die Versichertenrenten dar. Man erkennt, dass sich die Struktur dieser Zugänge in den vergangenen Jahren deutlich geändert hat: Seit 1996 lässt sich für die Renten wegen verminderter Erwerbsfähigkeit ein beständiger Rückgang feststellen. Trotzdem machten sie 2007 noch rd. ein Sechstel der Zugänge aus. 
Nachdem der Anteil der Zugänge in die Regelaltersrente bis in die späten 90er Jahre trotz einsetzender Bemühungen, vorzeitigen Renteneintritt unattraktiv zu gestalten, ständig zurückgegangen war, machte diese Zugangsart im Jahr 2007 wieder gut ein Drittel der Rentenzugänge aus. Unauffällig entwickelten sich die Zugänge in die Rente für langjährig Versicherte. Ihr Anteil sank seit dem Ende der 90er Jahre und liegt 2007 bei gut acht Prozent. Einen größeren Anteil am Zugangsgeschehen als 1996 machen 2007 die Altersrenten für schwer behinderte Menschen mit 8,4 Prozent aus. Ein besonders starker Rückgang ist für die Renten wegen Arbeitslosigkeit und nach Altersteilzeit zu verzeichnen. Nachdem ihr Anteil in den frühen 1990er Jahren sprunghaft angestiegen war, um in den Folgejahren beständig abzunehmen, gehen nun 2007 11,5 Prozent der Neurentner auf diesem Weg in den Ruhestand. Der Anstieg der Frauenrenten in den späten 90er Jahren auf kapp ein Viertel der Neurenten mag zum einen an einer gewachsenen Erwerbsbeteiligung von Frauen und einer damit einhergehenden höheren Quote an Rentenberechtigten dieser Zugangsart liegen, zum anderen an den Besonderheiten der geschlechtsspezifischen Erwerbsbiographien in der ehemaligen DDR. Auch hier hinterlässt die Einschränkung der Zugangsregeln seit 2000 Spuren in der Inanspruchnahme.

Tab. 3: $\quad$ Zugänge in die Versichertenrenten in \% nach Jahren

\begin{tabular}{|c|c|c|c|c|c|c|}
\hline Jahr & $\begin{array}{l}\text { Rente wegen } \\
\text { verminderter } \\
\text { Erwerbs- } \\
\text { fähigkeit }\end{array}$ & $\begin{array}{l}\text { Regel- } \\
\text { alters- } \\
\text { rente }\end{array}$ & $\begin{array}{l}\text { für langjäh- } \\
\text { rig Ver- } \\
\text { sicherte }\end{array}$ & $\begin{array}{l}\text { für Schwer- } \\
\text { behinderte }\end{array}$ & $\begin{array}{c}\text { wegen } \\
\text { Arbeitslosig- } \\
\text { keit/nach Al- } \\
\text { tersteilzeit }\end{array}$ & für Frauen \\
\hline 1996 & 24,7 & 24,3 & 7,3 & 4,3 & 19,8 & 19,5 \\
\hline 1997 & 24,0 & 22,6 & 8,2 & 4,5 & 19,9 & 20,8 \\
\hline 1998 & 22,6 & 22,8 & 9,3 & 4,8 & 17,4 & 22,9 \\
\hline 1999 & 19,9 & 24,4 & 9,3 & 5,0 & 16,8 & 24,5 \\
\hline 2000 & 19,6 & 26,3 & 8,5 & 6,0 & 18,1 & 21,5 \\
\hline 2001 & 19,7 & 27,7 & 8,1 & 6,9 & 16,6 & 20,9 \\
\hline 2002 & 18,6 & 30,3 & 8,8 & 7,3 & 15,5 & 19,5 \\
\hline 2003 & 17,4 & 33,2 & 8,5 & 7,3 & 14,5 & 19,1 \\
\hline 2004 & 17,3 & 35,2 & 7,7 & 7,3 & 13,5 & 18,9 \\
\hline 2005 & 17,5 & 36,4 & 7,9 & 7,0 & 14,5 & 16,7 \\
\hline 2006 & 17,4 & 37,7 & 8,2 & 7,3 & 12,4 & 17,0 \\
\hline 2007 & 18,7 & 35,8 & 8,1 & 8,4 & 11,5 & 17,5 \\
\hline
\end{tabular}

Quelle: DRV Bund (2008)

Die Zugangsregeln setzen starke und - wie oben zitiert - ausgiebig untersuchte ökonomische Anreize, die sich zudem im Zeitverlauf geändert haben. Deshalb ist es für die Analyse dringend notwendig, diese Anreize mit zu kontrollieren. Um sie realitätsnäher zu gestalten, haben sich allerdings einige Erweiterungen dieser Modelle entwickelt, von denen im Folgenden die Einbeziehung des familiären Kontexts und der Rolle der Gesundheit dargestellt werden. 


\section{A 1.1.2 Familiärer Kontext}

Zunehmende Bedeutung erfährt in der wissenschaftlichen Untersuchung des Übergangs aus dem Erwerbsleben die Modellierung des Haushaltskontextes und die Einbeziehung des Verhaltens des Partners. ${ }^{26}$ Mit zunehmender Erwerbsbeteiligung auch älterer Frauen spielen Überlegungen, gemeinsam den Ruhestand zu verbringen, aber auch Rentenansprüche des Partners zum Zeitpunkt der Entscheidung eine größere Rolle. Dabei gehen die Untersuchungen von der Vermutung aus, dass sich die mit der Verrentungsentscheidung eingehandelte Freizeit der Partner komplementär zueinander verhält. Die vorhandene Literatur findet dafür auch deutliche Anhaltspunkte: Für Deutschland zeigen Blau und Riphahn (1999), dass für verheiratete Paare die Erwerbsbeteiligung des Ehepartners einen positiven Einfluss auf die Entscheidung, Arbeit aufzunehmen, und einen negativen Einfluss auf die Entscheidung zur Frühverrentung ausübt. Der Effekt lässt sich auch für andere Länder nachweisen. So zeigen die Ergebnisse von Christensen und Datta Gupta (1999) aus der Retirement History Study (RHS) eine deutliche Tendenz zur gemeinsamen Renteneintritt von Partnern. ${ }^{27}$ Mastrogiacomo et al. (2004) untersuchen die gemeinsame Verrentungsentscheidung älterer Paare in den Niederlanden und kommen zu denselben Ergebnissen.

\section{A 1.1.3 Gesundheit}

Neben streng ökonomischen Einflussgrößen haben sich weitere Charakteristika der Beschäftigten als bedeutsam für die Arbeitsangebotsentscheidung Älterer herausgestellt, darunter der Gesundheitszustand. Theoretisch lässt sich dieser zum einen als Beschränkung der intertemporalen Budgetrestriktion in der simultanen Freizeit-Konsum-Entscheidung ausdrücken, zum anderen als Bestimmungsgröße für Präferenzbildung (vgl. Lumsdaine und Mitchell (1999), S. 3282f.). Eine Verschlechterung des Gesundheitszustandes verringert vielfach die Beschäftigungsfähigkeit und senkt damit die Erwerbsaussichten des Arbeitsanbieters. Solange der Substitutionseffekt Überhand über den Einkommenseffekt behält, verringert die Person bei einer negativen Änderung des Gesundheitszustandes sein Arbeitsangebot. Ein solches Ereignis kann in der intertemporalen Arbeitsangebotsentscheidung auch den Planungshorizont in Form einer verkürzten Lebenserwartung widerspiegeln und führt auch dann zu einem vorgezogenen Rückzug auf das Altenteil. Zudem lässt sich auch argumentieren, dass ein schlechter Gesundheitszustand das Arbeitsleid erhöht und daher eine Veränderung der Präferenzen bewirkt, sodass die Person einen früheren Renteneintritt vorzieht. Theoretisch lässt sich daher ein negativer Effekt einer sich verschlechternden Gesundheit auf das Arbeitsangebot vermuten.

\footnotetext{
${ }^{26}$ Vgl. z.B. die Arbeiten von Hurd (1990), Gustman und Steinmeier (1994) sowie Blau (1998).

${ }^{27}$ Ebenfalls für die USA kommt Coile (2003) zu dem Schluss, dass Männer sehr wohl, Frauen dagegen gar nicht von der Verrentungsentscheidung des Ehepartners beeinflusst sind.
} 
Viele Studien haben den Einfluss des gesundheitlichen Zustandes auf die Ruhestandsentscheidung hervorgehoben. Ausgehend von den ersten empirischen Arbeiten zum Erwerbsverlauf Älterer, die in den 80er Jahren Auswertungen der RHS für die USA vornahmen, hat sich die Erkenntnis durchgesetzt, dass die Gesundheit einen entscheidenden Einfluss auf die Ruhestandsentscheidung hat und gesundheitliche Probleme daher einen wichtigen Faktor zur Erklärung von Frühverrentung darstellen (vgl. Sammartino (1987), Rust (1989), Quinn et al. (1990)). Dabei kommen einige Forscher zu dem Schluss, dass gesundheitliche Variablen einen stärkeren Einfluss auf den Austritt ausüben als die finanziellen Rahmenbedingungen dieser Entscheidung (vgl. z.B. McGarry (2002)).

Unklarheit über die Einbeziehung von Gesundheitsvariablen besteht danach nur in methodischer Hinsicht: Wie verlässlich sind womöglich endogene Selbstauskünfte über den eigenen gesundheitlichen Zustand? Hintergrund ist die Beobachtung, dass Selbsteinschätzungen des eigenen Gesundheitszustandes meist deutlich negativer ausfallen, als es objektive Maße erwarten lassen (vgl. Bazzoli (1985)). Etliche Arbeiten kreisten daher um den Umgang mit solchen, möglicherweise systematisch fehlerbehafteten Angaben. Während eine Forschungsrichtung sich mit den Anforderungen an ein objektives Maß der Gesundheit bemühten (vgl. Anderson und Burkhauser (1985) oder Bound (1991)), versuchte eine andere, das Problem der Endogenität der selbst erklärten Variablen Gesundheit bei der Schätzung des Eintrittsereignisses (vgl. Dwyer und Mitchell (1998), Dwyer (2001) oder Larsen und Datta Gupta (2004)) zu berücksichtigen. Lindeboom und Kerkhofs (2002) argumentieren, dass selbst objektive Gesundheitsmaße endogen sind, da sie vom Erwerbsverlauf einer Person mitbestimmt sind und stellen ein Modell auf, in dem sowohl Erwerbsstatus als auch Gesundheitszustand endogenisiert sind und das sie anhand von niederländischen Querschnittsdaten testen. Dwyer (2001) kommt zu dem Schluss, dass weniger der Gesundheitszustand selbst als vielmehr schockartige Änderungen desselben die Erwerbsbeteiligung im Alter bestimmt.

Schließlich stellt - wie oben erwähnt - eine gesundheitliche Beeinträchtigung auch eine der Voraussetzungen dar, über die Erwerbsunfähigkeitsrente oder ein vorgezogene Altersrente frühzeitig aus dem Arbeitsleben auszuscheiden. Für die folgenden, empirischen Analysen ergibt sich daher die dringende Notwendigkeit, für diese Einflussgröße zu kontrollieren.

\section{A 1.2 Nachfrageseitige Bestimmungsgründe}

Die Entscheidung, von der Erwerbstätigkeit in den Ruhestand zu wechseln, ist eine Wahl, die - sei es direkt, sei es indirekt - auch von der Nachfrageseite des Arbeitsmarktes, und damit den betrieblichen Bedingungen abhängt. Sie ist daher nicht einzig abhängig von Präferenzen des Betroffenen, seinen Lebensumständen und den institutionellen Restriktionen des Rentensystems. Dass ältere Beschäftigte frühzeitig aus dem Erwerbsleben ausscheiden, mag auch von geänderten Anforderungen seitens des Betriebes an die Fähigkeiten der Mitarbeiter und damit seinen Erwerbsaussichten abhängen. Anders als in den meisten bisherigen Untersuchungen steht hier die Frage im Mittelpunkt des Interesses, ob und wie betriebliche Maßnah- 
men den Eintritt in die Rente zu beeinflussen vermögen. Im Folgenden sollen daher die wichtigsten Ansätze beschrieben werden, mit denen sich die Weiterbeschäftigung von Älteren durch ihre Betriebe erklären lässt. ${ }^{28}$

\section{A 1.2.1 Altersstruktur}

Im Kontext der Erforschung heterogener Arbeit, die sich zunächst stark mit qualifikatorischer Heterogenität des Faktors Arbeit beschäftigte, wird seit einigen Jahren die Nachfrage nach unterschiedlichen Altersgruppen näher untersucht. Anlass für eine solche Differenzierung ist die Tatsache, dass Junge und Alte sich in vielerlei Hinsicht strukturell unterscheiden, was den Einsatz dieser Gruppen im Produktionsprozess unterschiedlich attraktiv und daher eine Betrachtung dieser Dimension der Heterogenität angemessen erscheinen lässt. Dabei sind die Unterschiede sowohl auf Kohorten- als auch auf reine Alterseffekte zurückzuführen.

So lassen sich Kohorteneffekte ausmachen, die mit dem Geburtsjahrgang variieren. Dazu zählen unterschiedliche, für diese Jahrgänge typische Qualifikationsniveaus und Lerninhalte. Alterseffekte sind unabhängig von der Kohorte allein der Tatsache geschuldet, dass sich eine Person ein bestimmtes Lebensalter erreicht hat. Beispiele hierfür sind ein im Alter zunehmender Schatz an Erfahrungswissen, ein höheres gesundheitliches Risiko und ein Nachlassen der kognitiven und körperlichen Fähigkeiten, das in etlichen Studien nachgewiesen wurde. ${ }^{29}$

Der Befund ist allgemeingültig: Personen mit hohen kognitiven Fähigkeiten erleiden ebenso einen Abfall wie solche, die ohnehin schon geringer damit ausgestattet sind (vgl. Deary et al. (2000). ${ }^{30}$ Auch ließen sich weder zwischen Männern und Frauen Unterschiede in dieser Entwicklung feststellen (vgl. Maitland et al. (2000)) noch zwischen verschiedenen Kulturkreisen (vgl. Park et al. (1999)). Allerdings konnten Differenzen zwischen einzelnen kognitiven Fähigkeiten ermittelt werden, die im Alter nachlassen, während andere von der Alterung unbeeinflusst bleiben. Als erstere gelten fluide kognitive Fähigkeiten wie Kombinationsfähigkeit, Genauigkeit und Orientierung in neuen Situationen. $\mathrm{Zu}$ den altersunabhängigen, den so genannten kristallinen, zählen Allgemeinwissen, Wortschatz und Sprachverständnis (vgl. Horn und Cattell (1966) und (1967)). Diese Heterogenität spiegelt sich auch in den Einstellungen der Betriebe gegenüber Älteren wider, die 2002 im IAB-Betriebspanel erfragt wurden. Eine Auswertung von Strotmann und Hess (2003) für Baden-Württemberg ergibt, dass viele Betriebe die Einstellung von Älteren entweder gänzlich ablehnen und gleichzeitig deutliche Un-

\footnotetext{
${ }^{28}$ Dass neben den hier beschriebenen auch andere Determinanten die Nachfrage nach Älteren bestimmen, versteht sich von selbst. So weisen experimentelle Studien - zumindest für ausgewählte Branchen - eine negative Diskriminierung Älterer durch Personalverantwortliche nach (vgl. z.B. Riach und Rich (2006)).

${ }^{29}$ Einen Überblick über verschiedene Studien zum Zusammenhang zwischen Alter und Produktivität bieten Skirbekk (2003) und Börsch-Supan et al. (2005).

${ }^{30} \mathrm{Zu}$ diesem Ergebnis kommen auch Oster und Hamermesh (1998), die nachweisen, dass ältere Wirtschaftswissenschaftler weniger Journalartikel publizieren als jüngere. Ein Trend, der für führende Wissenschaftler ebenso gilt wie für andere.
} 
terschiede für einzelne Merkmale der Leistungsfähigkeit verschiedener Altersgruppen wahrnehmen, die sich mit den oben genannten Erkenntnissen decken.

Diese Erkenntnisse über den strukturellen Zusammenhang zwischen Alter und im Arbeitsprozess notwendigen Fähigkeiten rechtfertigen die Annahme, dass eine gesonderte Betrachtung der Nachfrage nach der Arbeitskraft Älterer angemessen und sinnvoll ist.

Die Debatte um altersspezifisches Nachfrageverhalten der Betriebe knüpft methodisch und inhaltlich an Untersuchungen, in denen der Einsatzfaktor Arbeit nicht mehr als einheitlich angenommen wird. Dabei wird betriebliche Nachfrage nach Älteren modelliert, indem man heterogene Arten der Arbeit als separate Inputfaktoren im Produktionsprozess interpretiert. Analog zur Analyse der Nachfrage nach Beschäftigten unterschiedlicher Qualifikation können dann Systeme der betrieblichen Nachfrage mikroökonomisch modelliert werden und Substitutions- und Komplementaritätsbeziehungen zwischen den Einsatzfaktoren geschätzt werden.

Eine erste Abhandlung zu dem Thema für die USA findet sich in Card und Lemieux (2001). Für Deutschland präsentieren Fitzenberger et al. (2003) erste Ergebnisse. Ausgehend von einer zweistufigen CES-Produktionsfunktion schätzen sie Substitutionselastizitäten zwischen Alters- und Qualifikationsgruppen auf Basis der Beschäftigtenstichprobe IABS. Beide Studien kommen zu dem Ergebnis, dass eine endliche Substitutionselastizität zwischen den Altersgruppen besteht. Diese stellen danach keine perfekten Substitute dar. Dabei können die Autoren Unterschiede der Substituierbarkeit je nach Qualifikation feststellen. Für die gering und hoch Qualifizierte ist diese relativ hoch, während sich Beschäftigte mit mittleren Ausbildungsniveaus aber unterschiedlichen Alters nur mit größerem Aufwand gegeneinander austauschen lassen.

Ein weiterer Forschungsstrang, der die Nachfrage nach Älteren durch die Altersstruktur zu erklären versucht, ist die Untersuchung der Verdrängung oder Ablösung Älterer durch Jüngere innerhalb eines Betriebes. ${ }^{31}$. Allart et al. (2005) kommen nach einer Analyse solcher Austausch-Prozesse älterer Beschäftigter durch Jüngere anhand von Daten des IABBetriebspanels zu dem Schluss, dass der Anteil Jüngerer im Betrieb die Austauschrate signifikant erhöht. Nach Blau und Shvydko (2007) senkt der betriebsspezifische Anteil Älterer die individuelle Wahrscheinlichkeit der Beendigung eines Arbeitsverhältnisses signifikant. Anhand eines Employer-employee-Datensatzes aus den USA belegen sie, dass in Betrieben mit einem höheren Anteil Älterer die Wahrscheinlichkeit einer Beendigung des Arbeitsverhältnisses geringer ist. Sie erklären dies mit Selbstselektionsprozessen der Beschäftigten: Ältere Arbeitnehmer wählen demnach eher solche Betriebe, die ihren Wünschen nach altersgerechter Organisation der Arbeit gerecht werden.

\footnotetext{
${ }^{31}$ Vgl. z.B. Bender, Preißler und Wübbeke (2000).
} 
Für den empirischen Teil dieses Kapitels lässt sich daraus der Schluss ziehen, dass die Altersstruktur des Betriebes eine mögliche Erklärung der Nachfrage nach Älteren darstellt und daher kontrolliert werden muss.

\section{A 1.2.2 Anpassungskosten}

Viele Unternehmen nutzen natürliche Fluktuation wie sie auch die (Früh-) Verrentungsentscheidung darstellt, um Anpassungen des Personalbestands kostengünstig vorzunehmen (vgl. Gatter (2004)). Es ist deshalb davon auszugehen, dass je nach Anpassungskostenprofil des Betriebes vorzeitiger Rentenantritt als personalpolitisches Instrument genutzt wird. Ein geringer Anteil an befristet Beschäftigten, an Beschäftigten in der Probezeit und aus Leiharbeit sprächen nach dieser Hypothese für eine höhere Wahrscheinlichkeit einer Frühverrentung. Als Alternative zur Frühverrentung bietet sich allerdings auch die Altersteilzeit an, der die Arbeitgeberseite mit einer Betriebsvereinbarung zustimmen muss. Entschließen sich die Entscheidungsträger eines Betriebes, ihren Beschäftigten ein Altersteilzeitmodell anzubieten, sinkt c.p. die Wahrscheinlichkeit, dass ein Beschäftigter frühzeitig die Rente antritt wegen der attraktiven Alternative zur Frührente. Boockmann und Zwick (2004), die den Anteil Älterer im Betrieb schätzen, finden einen negativen Einfluss des Anteils der Auszubildenden auf die Abhängige und relativieren damit das Argument der Schutzfunktion von Randbelegschaften für den Kern.

Es drängt sich also auf, in die folgende Verweildaueranalyse Merkmale mit aufzunehmen, die die Möglichkeiten des Betriebes abbilden, kostengünstig Anpassungen an eine verringerte Arbeitsnachfrage vorzunehmen.

\section{A 1.2.3 Jobcharakteristika}

Neben den Charakteristika des Betriebes müssen auch solche Einflüsse berücksichtigt werden, berufliche Einflussgrößen, die eher den Arbeitsplatz kennzeichnen als den Betrieb, in dem die Arbeit verrichtet wird.

Bartel (1982) konnte in ihrer Studie zum Austrittsverhalten aus dem Arbeitsmarkt keine beruflichen Bestimmungsgründe für den Zeitpunkt der Verrentung ausmachen: Anhand von Daten des National Longitudinal Survey of Older Men kommt sie zu dem Schluss, dass weder sich wiederholende Aufgaben, noch körperlich anspruchsvolle Tätigkeiten oder ,schlechte Arbeitsbedingungen“ entscheidenden Einfluss auf die Austrittswahrscheinlichkeit ausüben. Ebenso wenig konnte Quinn (1977) Jobcharakteristika als wichtige Bestimmungsgründe für die untersuchte Fragestellung ausmachen. Weder geringe Eigenständigkeit, noch Stress beieinflussten die Wahrscheinlichkeit signifikant.

Zwar gelingt es in neueren Arbeiten, Personen mit nachteiligen Arbeitsplatzeinflüssen eine höhere Neigung zu früherem Renteneintritt nachzuweisen, doch die Höhe des Einflusses ist weiterhin umstritten. So kommt die Studie von Gustman und Steinmeier (1986), die sich auf 
Daten der RHS bezieht, zu dem Schluss, dass physisch anstrengende Arbeit einen früheren Einstieg in den Ruhestand begünstigt. Allerdings stehen dem Schluss methodische Probleme entgegen, den singulären Effekt dieses Arbeitsplatzcharakteristikums zu trennen von einem indirekten Effekt über die Gesundheit des Beschäftigten (vgl. auch Lumsdaine und Mitchell (1999), S. 3293). Zu einem ähnlichen Ergebnis kommen Hurd und McGarry (1993), die darauf hinweisen, dass ein möglicher Wechsel auf einen ruhigeren Job beim selben Arbeitgeber das erwartete Austrittsalter erhöht.

\section{A 1.2.4 Institutionen}

Ebenso wie Arbeitsanbieter sind auch die Unternehmen in ihrer Entscheidung zur (Weiter-) Beschäftigung von Älteren nicht gänzlich frei von gesetzlichen und anderen institutionellen Zwängen. Etliche Regelungen begrenzen den Handlungsspielraum der unternehmerischen Entscheidungsträger: betriebliche Mitbestimmung, Senioritätsentlohnung, sowie Kündigungsschutz und (haus-) tarifliche Kündigungsverbote. Zudem können staatliche Beihilfen, die die Neueinstellung bzw. Weiterbeschäftigung Älterer fördern, Unternehmen in ihrer Personalpolitik beeinflussen. Im Folgenden sollen die wichtigsten dieser institutionellen Mechanismen vorgestellt werden.

\section{A 1.2.4.1 Mitbestimmung}

Die betriebliche Mitbestimmung ist in Deutschland im Betriebsverfassungsgesetz (BetrVG) niedergelegt. Danach kann ein Betriebsrat in Betrieben mit mindestens fünf ständigen, wahlberechtigten Arbeitnehmern gegründet werden. Das BetrVG regelt auch die Rechte des Betriebsrats, dem darin ein Informations- und Mitwirkungsrecht im Falle von personellen Maßnahmen wie Kündigungen zusteht. In dieser Rolle kann die Interessenvertretung Einfluss auf die Alterszusammensetzung und die Beschäftigungsaussichten von Älteren nehmen.

Einige Studien lassen in der Existenz einer Arbeitnehmervertretung positive Aspekte für die Situation Älterer im Betrieb erkennen, auch wenn sie nicht immer explizit diese Gruppe adressieren: So finden Frick und Sadowski (1995) heraus, dass die Existenz eines Betriebsrates die Mitarbeiterfluktuation verringert. Addison et al. (2001) bestätigen diese Befunde. Nach ihren Ergebnissen wirkt sich eine Interessenvertretung zudem auch dämpfend auf die Zahl der Abgänge im Betrieb aus. Auch nach den Ergebnissen von Beckmann (2002) geht von Organen der Mitbestimmung ein positiver Einfluss auf den Anteil der Über-50-Jährigen aus. In ihrer Untersuchung der Altersstruktur von deutschen Betrieben kommen Boockmann und Zwick (2004) zu dem Ergebnis, dass mit dem Vorhandensein von Betriebsräten der Anteil Älterer c.p. steigt. Auch sie erklären diesen Befund mit vermuteten und mit einem Betriebsrat verbundenen Erschwernissen, ältere Mitarbeiter durch Jüngere zu ersetzen.

Im Gegensatz dazu kommt Wübbeke (1999) in ihrer Analyse zu dem Schluss, dass der Einfluss des Betriebsrates sich darin äußert, dass Betriebe statt Kündigungen vorzunehmen eher 
Ältere in einen frühzeitigen Ruhestand drängen, da dieser als sozial verträglicher angesehen wird.

\section{A 1.2.4.2 Tarifbindung}

Einen Anhaltspunkt zur Bestimmung der Verbleibewahrscheinlichkeit im Arbeitsleben könnte auch der Umstand bieten, ob der beschäftigende Betrieb sich einem Arbeitgeberverband angeschlossen hat und damit den Bestimmungen des für ihn geltenden Tarifvertrages unterliegt. Die Tarifbindung eines Unternehmens hat mitunter direkte Auswirkungen auf Entlohnung, Arbeitszeit und Arbeitsorganisation der beschäftigten Älteren. ${ }^{32}$ Mit ihrer Koppelung von Tarifentgelten an Betriebszugehörigkeitsdauer und Alter garantieren entsprechende Regelungen der Stammbelegschaft Lohnzuwächse. Auch sehen einige Vereinbarungen das Instrument der Verdienstsicherung vor, das Älteren ein bereits erreichtes Lohnniveau zumindest zeitweise sichert und vor Abgruppierung schützt. Weiterhin sind auch altersgestaffelte Arbeitszeiten Gegenstand von Tarifverträgen. Seit 1996 verpflichten sich Unternehmen, ihren Beschäftigten Altersteilzeit anzubieten, sowie Verdienst und Rentenbeiträge aufzustocken. In Einzelfällen sehen Tarifverträge auch altersspezifische Arbeitszeiten vor mit einer geringeren Wochenstundenzahl oder mehr Urlaubstagen für Ältere. Am weit reichendsten greifen Kündigungsverbote von Älteren in die unternehmerische Entscheidungsfreiheit ein. Sie sind Teil vieler Tarifverträge und erhöhen sicher die Kosten auch gütlicher Trennungen vom Arbeitnehmer. Die Wirkung dieser Aspekte von Tarifbindung auf die Erwerbschancen Älterer ist nachfragetheoretisch zunächst unbestimmt: Einerseits treiben sie die Arbeitskosten Älterer in die Höhe, andererseits erschweren sie die Entlassung. Für die begünstigten Arbeitnehmer werden die im Vertrag enthaltenen Regeln einen Anreiz zur Verlängerung ihrer Erwerbstätigkeit darstellen.

Boockmann und Zwick (2004) argumentieren, dass der von tarifvertraglichen Verpflichtungen ausgehende Effekt mittelbar über die damit verbundene Entlohnung der älteren Beschäftigten wirkt. In ihrer Analyse der Altersstruktur baden-württembergischer Betriebe des IABBetriebspanels kommen sie allerdings nicht auf signifikante Einflüsse der Tarifbindung.

\section{A 1.2.4.3 Senioritätsentlohnung}

Eine häufig genannte Erklärung für die geringe Erwerbsquote Älterer ist die gängige und teilweise institutionalisierte Praxis der Senioritätsentlohnung. Sie stellt eine weitere, auch dem institutionellen Umfeld des Arbeitsmarktes zuzurechnende Einschränkung unternehmerischen Handelns dar.

Der Zusammenhang zwischen Entlohnung und Alter ist ebenso oft dargestellt wie gut erforscht. ${ }^{33}$ Dabei steigt die Entlohnung kontinuierlich bis in das sechste Lebensjahrzehnt, um

\footnotetext{
${ }^{32}$ Einen Überblick über die altersspezifischen Regelungen von Tarifverträgen liefert Bispinck (2002).

${ }^{33}$ Für eine neuere Quelle für Deutschland vgl. Haan und Steiner (2006) S. 4.
} 
danach bis zum Eintritt in die Rente leicht abzunehmen. Als Erklärungsansätze für Senioritätsentlohnung lassen sich zum einen institutionelle Gründe anführen, zum anderen lässt sich die Beobachtung auch aus einem freiwilligen betrieblichen Verhalten heraus erklären.

Vergütungsregeln des öffentlichen Dienstes z.B. sehen eine mit dem Alter steigende Entlohnung vor. Aber auch andere Wirtschaftszweige kennen eine formalisierte Form der Senioritätsentlohnung, die oft in Tarifverträgen und Betriebsvereinbarungen festgeschrieben ist. So ist es für gewerblich Beschäftigte oft erst mit einer gewissen Berufserfahrung möglich, in höhere Lohngruppen vorzustoßen. Eine automatische Höhergruppierung ist nur in einer Handvoll Branchentarifverträgen verankert. Eine direkte Koppelung von Lebensalter und Gehaltsgruppe ist lediglich bei jüngeren Angestellten festzustellen (vgl. Bispinck (2002), S. 6ff.). Darüber hinaus enthält eine Vielzahl von Tarifverträgen Regelungen zur Verdienstsicherung und zu Abfindungen, die an ein Mindestalter und/oder an eine Mindestbetriebszugehörigkeitsdauer gekoppelt sind. Darin wird das aktuelle Verdienstniveau für Fälle abgesichert, die nicht im Einflussbereich des Beschäftigten stehen. So müssen die durch den Vertrag geschützten Arbeiter und Angestellten als Folge altersbedingter Leistungsminderung oder von Rationalisierungen keine Abgruppierungen oder Lohneinbußen fürchten (vgl. Bispinck (2002) S. 8f.).

Allerdings lässt sich Senioritätsentlohnung auch aus einem unternehmerischen rationalen Kalkül heraus erklären, ohne dass auf den Einfluss von außen vorgegebener Restriktionen zurückgegriffen werden müsste. Zum einen erklärt die Akkumulation von insbesondere betriebsspezifischem Humankapital die Lohnentwicklung. Darüber hinaus beschäftigt Ökonomen aber die Frage, wie es zu Disparitäten zwischen Lohn- und Produktivitätsentwicklung über den Erwerbsverlauf hinweg kommt. ${ }^{34}$ Die Beobachtung, dass Entlohnung in jungen Jahren unter, später über der Produktivität liegt, bildet die Grundlage für die These von Lazear (1979). Er erklärt den Befund mit sich daraus ergebenden Anreizen und sieht die Lohnentwicklung als eine Form nachträglicher Vergütung, die das Entlassungsrisiko finanziell bedeutsam macht und so zur Disziplinierung der Angestellten beiträgt. ${ }^{35}$

Loewenstein und Sicherman (1991) gehen einer weiteren Alternativerklärung nach, die sich aus den Präferenzen der Beschäftigten ergibt: Danach ziehen sie einen ansteigenden Zah-

\footnotetext{
${ }^{34}$ Vertreter der Humankapitaltheorie argumentieren, dass die individuelle Lohnentwicklung durch den Zuwachs an Erfahrungswissen, durch Lerneffekte und Weiterbildung zu erklären sei (vgl. Becker (1964), Mincer (1974) und Mortensen (1978)). Neuere Forschungen lassen allerdings erkennen, dass allein durch vermutete Produktivitätssteigerungen nicht zu rechtfertigen sind. Die vorgestellten Theorien ergaben sich aus dieser Vermutung.

${ }^{35}$ Dies bedarf langfristiger Arbeitsverträge und einem von vornherein festgelegten Renteneintrittsalter und beantwortet die im Titel gestellte Frage „why is there mandatory retirement?“. Die These wurde verschiedentlich empirischen Tests unterzogen (vgl. Hutchens (1986), Hutchens (1987), Leigh (1984) und Heywood et al. (1999)). So stellt Hutchens (1987) fest, dass Beschäftigte mit leicht zu kontrollierenden Tätigkeiten in geringerem Maße von starren Verrentungsregeln betroffen sind, wesentlich weniger Lohn erhalten und eine geringere Dauer ihrer Arbeitsverträge aufwiesen. Heywood et al. (1999) führen die Beobachtung geringerer Einstellungschancen von Älteren mittels einer empirischen Untersuchung auf mangelnde Möglichkeiten zurück, Leistungsanreize durch die Aussicht auf einen späteren Lohnanstieg zu setzen.
} 
lungsstrom einem konstanten vor, auch wenn dieser dem Barwert nach geringer ist. Sie führen diese Ergebnisse einer eigenen Erhebung auf antizipierte mangelnde Selbstkontrolle und Verlustaversion der Probanden zurück.

\section{A 1.2.4.4 Altersdiskriminierung im Kündigungsschutz}

Im Falle von anstehenden Entlassungen erfahren ältere Arbeitnehmer ebenfalls eine Sonderbehandlung. Sowohl arbeitsrechtliche wie tarifvertragliche Bestimmungen stellen die Gruppe unter einen besonderen Schutz.

So sieht das Kündigungsschutzgesetz (KSchG) vor, dass das kündigende Unternehmen im Falle betriebsbedingter Kündigungen eine so genannte Sozialauswahl treffen muss. Danach dürfen lange beschäftigte Ältere zunächst nicht entlassen werden. Nachfragewirksam können diese Regelungen dann werden, wenn sie die erwarteten Anpassungskosten eines Unternehmens erhöhen. So kann eine Kombination aus fortgesetzter Lohnzahlung bis zur arbeitsgerichtlichen Entscheidung und einer Abfindungszahlung, sich leicht zu einem Jahreslohn aufsummieren.

Auch die im BGB verankerte Regelung zur Kündigung sieht implizit eine Sonderbehandlung Älterer vor. Zwar enthält sie keinen ausdrücklichen Verweis auf das Alter der Beschäftigten, die Kündigungsfristen hängen aber von der Dauer der Betriebszugehörigkeit ab dem 25. Lebensjahr ab. Implizit sind damit ältere Beschäftigte schon von der BGB-Regelung begünstigt.

Zuletzt enthalten viele Tarifverträge und Betriebsvereinbarungen Kündigungsverbote, die an Altersgrenzen von 50, 53 oder 55 Jahren gekoppelt sind. Oder aber sie sehen hohe Abfindungszahlungen vor, die bis zu 18 Monatsentgelten entsprechen und an ein Mindestalter von i.d.R. 55 bis 60 Jahren gebunden sind. ${ }^{36}$

Gleichzeitig kann Kündigungsschutz jedoch Anreize auch dazu bieten, durch betriebliche Weiterbildung firmenspezifisches Humankapital zu entwickeln (vgl. Estevez-Abe et al. (2001)), und so den Druck zum frühzeitigen Ausscheiden aus dem Betrieb verringern.

Empirische Studien zum Einfluss von Kündigungsschutz auf die Beschäftigungssituation von Älteren sind rar. Die Bestimmung der Auswirkungen des Kündigungsschutzgesetzes und insbesondere seines Schwellenwertes wird daher auch Gegenstand einer eingehenden Untersuchung in Teil B dieser Arbeit sein.

\section{A 1.2.4.5 Altersdiskriminierung in der Sozialgesetzgebung}

Mit dem Auslaufen der vorgezogenen Renteneintrittsmöglichkeiten ergriff die Bundesregierung Maßnahmen, um die Altersarbeitslosigkeit zu bekämpfen. Beschäftigungs- und Wiedereingliederungschancen Älterer sollten erhöht und bestehende Beschäftigung gesichert werden.

\footnotetext{
${ }^{36}$ Einen Eindruck von der Vielfalt der Regelungen erhält man bei Bispinck (2002) S. 19ff.
} 
Insbesondere das Job-Aqtiv-Gesetz im Jahr 2002 und die Gesetze zur „Modernisierung des Arbeitsmarktes“ im Jahr 2003 brachten Neuerungen, die gerade in den Arbeitsmarkt für Ältere eingreifen sollten. Sie sollen hier erläutert werden.

Beitragsbefreiung: Im Rahmen der ersten Hartz-Gesetze ist die unbefristete Beitragsbefreiung von der Arbeitslosenversicherung neu eingeführt worden für neu eingestellte, zuvor arbeitslose Personen über 55 Jahren. Die Befreiung bezieht sich nur auf den Arbeitgeberanteil und machte bei Einführung der Maßnahme 3,25\% des Bruttolohnes aus. Die Regelung war zunächst auf Neueinstellungen bis zum 31.12. 2005 befristet, wurde aber verlängert. Allerdings hielt sich die Inanspruchnahme der Maßnahme in Grenzen. Für das Jahr 2003 wurden in knapp 7500 Fällen Arbeitgeber von ihren Beiträgen zur Arbeitslosenversicherung befreit. Eine Evaluation dieser Maßnahme erbrachte keine signifikante Steigerung der Wiedereinstellungschancen von Älteren (vgl. ZEW (2005), S. 302 ff.).

Entgeltsicherung: Ebenfalls im Gesetzespaket zur „Modernisierung des Arbeitsmarktes“ enthalten war eine zum 1.1. 2003 neu eingeführte Entgeltsicherung für Ältere (§ 421k SGB III). Danach können 50-Jährige und Ältere, die aus der Arbeitslosigkeit oder aus drohender Arbeitslosigkeit heraus eine geringer entlohnte Stelle annehmen und Anspruch auf Arbeitslosengeld von mind. 180 Tagen hätten, vom Arbeitsamt einen Zuschuss erhalten. Dieser beträgt $50 \%$ der Differenz zwischen dem letzten und dem neuen pauschalierten Nettoentgelt. Zudem erhalten die Bezieher eine Aufstockung ihrer Einzahlungen in die gesetzliche Rentenversicherung auf $90 \%$ des Betrages ihres letzten Entgelts. Die Förderungsdauer ergibt sich aus der verbleibenden Anspruchszeit auf Arbeitslosengeld I. Die Frist für die Antragstellung war zunächst bis Ende 2005 begrenzt und wurde später ausgedehnt.

Eine empirische Überprüfung des Förderinstruments im Rahmen der Evaluation der HartzGesetze ergab, dass nur für rd. $1 \%$ der neu aufgenommen Beschäftigungsverhältnisse von Personen zwischen 50 und 64 Jahren im Jahr 2003 eine solche Entgeltsicherung beantragt wurde. (vgl. Brussig et al. (2006), S. 5). Darüber hinaus ging nur ein geringer Anteil der Geförderten direkt aus einer Beschäftigung in die Maßnahme über (vgl. ZEW et al. (2005) S. 295). Auch angesichts der wenigen Förderfälle konnten keine signifikanten Beschäftigungseffekte für diese Form der aktiven Arbeitsmarktpolitik ermittelt werden (vgl. ZEW et al. (2005) S. 294ff.).

Eingliederungszuschuss: Eingliederungszuschüsse an den Arbeitgeber bei der Neueinstellung von Älteren gab es schon seit 1998. Allerdings war die Förderung zunächst beschränkt auf Langzeitarbeitslose ab einem Alter von 55 Jahren. Zum 1.8.1999 wurde die Anforderung an das Kriterium der Langzeitarbeitslosigkeit gelockert und die Altersgrenze auf 50 Jahre ge- 
senkt. Im Rahmen des Job-Aqtiv-Gesetzes zu Beginn des Jahres 2002 wurde der Kreis der Geförderten auf alle Arbeitnehmer über 50 Jahre ausgeweitet. ${ }^{37}$

Nach $\S 217$ SGB III können Arbeitgeber ,zur Eingliederung von Arbeitnehmern mit Vermittlungshemmnissen Zuschüsse zu den Arbeitsentgelten erhalten, wenn deren Vermittlung wegen in ihrer Person liegender Umstände erschwert ist." Dabei richten sich Förderhöhe und -dauer nach dem Umfang einer Minderleistung des Arbeitnehmers und nach den jeweiligen Eingliederungserfordernissen. Die Regelförderung für ältere Arbeitnehmer enthielt zunächst einen maximalen Lohnkostenzuschuss von 50\% für bis zu 24 Monate, mit Begründung betrug der Zuschuss bis zu 70\% für maximal 60 Monate. Die Förderung war jedoch zurückzuzahlen, wenn das Beschäftigungsverhältnis während der Förderung oder kurz danach beendet wurde. Diese Rückzahlungspflicht wurde zum 1.8.1999 aufgehoben. Das Hartz-III-Gesetz ordnete die Eingliederungszuschüsse Anfang 2004 neu. Im Rahmen dieser Neuordnung wurde der Zuschuss auf maximal 50\% und die Höchstförderungsdauer bei der Einstellung Älterer auf 36 Monate gekürzt, wobei der Fördersatz nach 12 Monaten gesenkt wird.

Die Evaluation dieser Förderung hat ergeben, dass Eingliederungszuschüsse einen positiven Effekt auf die Beschäftigung in der Gruppe der betrachteten Förderberechtigten ausüben. So kommen rd. 2 Prozent der Berechtigten innerhalb der ersten sechs Monate zu einem neuen Beschäftigungsverhältnis. Allerdings variiert die Wirkung der Förderung: Positive Effekte lassen sich für Ostdeutschland und Frauen nachweisen (vgl. ZEW et al. (2005) S. 274 ff.).

Qualifizierungsmaßnahmen: Seit Januar 2002 können Arbeitnehmern ab 50 Jahren die Kosten für die Teilnahme an Weiterbildungsmaßnahmen nach $§ 417$ SGB III erstattet werden, wenn sie in einem Betrieb mit bis zu 100 Beschäftigten angestellt sind, die Maßnahmen außerhalb des eigenen Betriebes stattfinden und ,über ausschließlich arbeitsplatzbezogene kurzfristige Anpassungsfortbildungen hinausgehen“. Zugleich kann der Arbeitgeber während der Weiterbildung eines von Arbeitslosigkeit bedrohten Arbeitnehmers einen Zuschuss zum Arbeitsentgelt als Ausgleich für Zeiten ohne Arbeitsleistung erhalten. Allerdings wurde diese Art der Förderung bisher kaum in Anspruch genommen (vgl. Eichhorst und Sproß (2005) S. 4).

Befristung: Eine weitere Maßnahme, die Chancen Älterer auf die Wiedereinstellung zu erhöhen, stellt $\S 14$ Abs. 3 des Teilzeit- und Befristungsgesetzes dar. Seit Anfang 2003 und zunächst bis Ende 2006 können Arbeitnehmer statt ab 58 Jahren bereits ab 52 Jahren ohne sachlichen Grund befristet beschäftigt werden, was für jüngere Arbeitnehmer nur maximal zwei Jahre lang zulässig ist. Zusammen mit der ohnehin möglichen Befristung von zwei Jahren ohne sachlichen Grund, konnten seit diesem Zeitpunkt Arbeitskräfte bereits ab 50 Jahren ohne Beschränkungen mehrfach hintereinander befristet eingestellt werden.

\footnotetext{
${ }^{37}$ Einen Überblick über die rechtlichen Änderungen für den Bezug von Eingliederungszuschüsse bieten ZEW et al. (2005) S. 440ff.
} 
Die Analyse dieser Regelung hat bislang keine beschäftigungsfördernde Wirkung zu Tage gefördert. Allerdings weisen die Autoren auf die kurze Geltungszeit der Neuregelung hin (vgl. Deutscher Bundestag (2006) S. 212 ff.).

\section{A 1.2.5 Technischer Wandel}

Wie sich technischer Fortschritt auf den Arbeitsmarkt und seine Segmente auswirkt, ist Forschungsgegenstand ganzer Generationen von Wissenschaftlern. Auch bei der Entscheidung über die Erwerbsbeteiligung Älterer und der Nachfrage nach Älteren können technischer Fortschritt und damit einhergehende Änderungen im Anforderungsprofil der Betriebe eine wichtige Rolle spielen.

Seit einigen Jahren wird der Einzug neuer Technologien, insbesondere der Informations- und Kommunikationstechnologie (IKT), in der Literatur in seinen Auswirkungen auf Arbeitsnachfrage und Entlohnung heftig diskutiert. ${ }^{38}$ Besondere Aufmerksamkeit wurde der Frage nach den Auswirkungen des Einzuges von Computern in die Arbeitswelt auf ältere Beschäftigte geschenkt: Hintergrund ist die Vermutung, dass erstens das Humankapital Älterer stärker abgewertet wird als das Jüngerer, und dass sich zweitens Ältere den durch die Technik veränderten Arbeitsanforderungen in geringerem Maße anpassen können und sich für diese Altersgruppe ein Einarbeiten in die neue Arbeitsweise aufgrund der geringeren Nutzungszeit des Wissens weniger lohnt. Dies, so die Vermutung, führt zu einem geringeren Einsatz von Computern durch Ältere, geringerer Produktivität dieser Gruppe und in Folge zu einer Senkung der Erwerbsaussichten, was einen Anreiz zum früheren Austritt böte. Die Forschungsarbeiten zu dem Thema lassen sich grob in zwei Fragestellungen unterteilen: Während sich die eine Gruppe mit den Bestimmungsgründen für die Verwendung moderner Technik am Arbeitsplatz befasst, liegt ein anderes Ziel darin, den Einfluss von Computernutzung auf Entlohnung und Erwerbsstatus zu schätzen. ${ }^{39}$

\footnotetext{
${ }^{38}$ Ausgangspunkt ist die Vermutung, dass der Einsatz solcher Technologien die Anforderungen an die Qualifikation der Beschäftigten erhöht. Dabei wird eine Komplementarität mit hoch qualifizierter Arbeit und eine substitutionale Beziehung zu gering Qualifizierten unterstellt. Als grundlegend kann die Arbeit von Kruger (1993) angesehen werden, an die sich eine weite Diskussion anschloss (vgl. z.B. DiNardo und Pischke (1997)). Die Ergebnisse dieser Arbeiten legen nahe, dass Hochqualifizierte häufiger IK-Technologien am Arbeitplatz einsetzen und dadurch höhere Löhne erzielen. Levy, Murnane (1996) und Autor et al. (2001) haben die Komplementarität von hoch qualifizierter Arbeit und Computereinsatz untersucht und kommen zu dem Schluss, dass der Einzug von IK-Technologien den Bedarf an hoch qualifizierten Beschäftigten erhöht. Einen Überblick über die Diskussion bieten Chennells und Van Reenen (2002). Die These des "digital divide“ scheint danach bestätigt.

${ }^{39}$ Nach der Studie von Weinberg (2001) wirken Arbeitserfahrung und Alter in entgegen gesetzter Weise auf den Gebrauch von Computern. Nach einer Untersuchung des Adaptionsverhaltens in den USA zwischen 1970 und 1997 schließt er, dass durch Erfahrung erworbenes Humankapital die Anwendung von Computern begünstigt, mit zunehmendem Alter aber die Fähigkeit zur Aneignung des notwendigen Wissens abnimmt. Die Folgerungen decken sich mit der Beobachtung von Friedberg (2003), die feststellt, dass die Altergruppe der 30- bis 49Jährigen den höchsten Anteil an Computernutzern aufweist. Ihr Ergebnis lautet allerdings, dass nicht Alter, sondern bevor-stehende Verrentung ältere Beschäftigte daran hindert, neue Technologien anzuwenden.
}

Auch Borghans und ter Weel (2002) untersuchen die Bestimmungsgründe für den individuellen Einsatz von Computern am Arbeitsplatz. Sie stützen sich in ihrer Analyse auf Daten des Skills Survey of the Employed 
Eine erste Studie, die sich direkt mit technischem Wandel und dem Rentenzugangsverhalten von älteren Arbeitern befasst, ist die Arbeit von Bartel und Sicherman (1993). Darin ziehen die beiden Autoren Daten von Männern im Alter von 45 bis 59 Jahren im Zeitraum von 1966 bis 1983 zu Rate. Das Resultat ist zweischneidig: Zum einen kommen sie zu dem Ergebnis, dass Arbeitnehmer, deren Branchen stärker von technologischem Wandel betroffen sind, ihren Renteneintritt länger als andere hinauszögern. Bartel und Sicherman sprechen dies dem Umstand zu, dass die neuen Techniken stärker zu Weiterbildungsaktivitäten veranlassen, was sich günstig auf Beschäftigungsmöglichkeiten und Arbeitseinkommen auswirkt und daher den Verbleib in der Erwerbstätigkeit verlängert. Zum anderen begünstigt überraschender technischer Wandel einen früheren Übergang in den Ruhestand, was die Autoren dem damit verbundenen raschen Abbau von Humankapital zuschreiben.

Friedberg (2003) untersucht den Einfluss von Computerverwendung am Arbeitsplatz auf die Renteneintrittsentscheidung. Anhand von Daten des CPS und der HRS aus den 90er Jahren schätzt sie den Übergang in die Rente in Abhängigkeit der Computernutzung mittels einer IVRegression. Sie kommt zu dem Ergebnis, dass Computer am Arbeitsplatz c.p. die Entscheidung für den Renteneintritt hinauszögern.

Für Deutschland liegen inzwischen auch empirische Arbeiten zu dem Thema vor. Um die Auswirkungen von IT-Nutzung auf die Arbeitsmarktposition von Älteren dreht sich die Arbeit von Bertschek (2004). Datengrundlage ist eine Erhebung des ZEW aus dem Jahr 2002 zur Nutzung und Verbreitung von IKT im verarbeitenden Gewerbe und in ausgewählten Dienstleistungssektoren mit knapp 4000 auswertbaren Beobachtungen. Befragt wurden Unternehmen mit mehr als vier Beschäftigten. ${ }^{40}$ Bertschek schätzt den Anteil der Beschäftigten im Alter von 50 Jahren und älter mit einem „fractional response“-Modell und kommt zu dem Ergebnis, dass der Anteil der Beschäftigten mit PC-Arbeitsplatz einen signifikant negativen Einfluss auf die abhängige Variable ausübt.

\footnotetext{
Workforce, in dem knapp 2500 Arbeiter im Alter von 20 bis 60 Jahren zur Computernutzung am Arbeitsplatz befragt wurden. Danach bestimmen Lohn und Geschlecht, nicht aber die Qualifikationsmerkmale der Angestellten die Nutzung. Auch der Regressor „Alter“ bleibt insignifikant. Die Autoren widersprechen damit der These von Friedberg, wonach bevorstehender Ruhestand die Aneignung neuer Technik behindert.

Beiden Arbeiten wird allerdings der Einwand der Selektionsverzerrung entgegengehalten: Da sie sich auf Daten Erwerbstätiger berufen, könnte ein mögliches, frühzeitiges Ausscheiden aus dem Betrieb korreliert sein mit dem Umstand, dass die inzwischen Verrenteten, nicht in der Studie Erfassten mit den Anforderungen moderner Computertechnik nicht Schritt halten konnten.

${ }^{40}$ Besonderes Augenmerk lag bei der Erhebung auf der Erfassung von Computernutzung am Arbeitsplatz. So wurde der Anteil der Beschäftigten erfragt, die den überwiegenden Anteil ihrer Tätigkeit am Rechner verbringen. Darüber hinaus wurden verschiedene Einsatzgebiete von ITK-Technik im Unternehmen erhoben: Danach hatte sich im Jahr 2002 die Nutzung von Computern am Arbeitsplatz schon stark durchgesetzt: rd. 53\% der Beschäftigten in den befragten Betrieben verrichteten den überwiegenden Teil ihrer Arbeitszeit vor dem Computerbildschirm, 98\% der Betriebe hielten einen Internetzugang bereit, gut 75\% verwendeten Software für Controlling, Planung und Steuerung.
} 
Schleife (2006) stützt sich in ihrer Untersuchung auf SOEP-Daten von Männern, die im Jahr 1997 vollzeitbeschäftigt und zwischen 46 und 60 Jahre alt waren. Nach Kontrolle von demografischen und betrieblichen Effekten hat nach ihren Ergebnissen die Nutzung von Computern am Arbeitsplatz keinen Einfluss auf die Verbleibewahrscheinlichkeit in Vollzeitbeschäftigung.

Hujer und Radic (2005) nutzen in ihrer Untersuchung ebenfalls den Linked Employer Employee Datensatz des IAB (LIAB), indem sie das IAB-Betriebspanel der Jahre 1993 bis 1997 um Informationen über die Alters- und Qualifikationsstruktur des Betriebs anreichern. In Matching-Ansätzen untersuchen sie, ob und wie sich die Einführung von Produkt- und Prozessinnovationen, sowie organisatorische Änderungen auf die Zusammensetzung des Betriebes auswirken. Sie kommen zu dem Ergebnis, dass technischer Wandel zwar die Älteren insofern benachteiligt, als ihr Beschäftigtenanteil im Betrieb aufgrund solcher Innovationen sinkt. Allerdings wird dieser Effekt geringer, sobald eine Unterscheidung bezüglich der Qualifikationsstruktur der Altersgruppen vorgenommen wird. Sie weisen zusammenfassend darauf hin, dass nachteilige Auswirkungen von Neuerungen der Arbeitsabläufe auf die Älteren eines Betriebes getrieben sind von einem durchschnittlich niedrigeren Qualifikationsniveau dieser Personen. So wirken die Änderungen an sich nicht altersdiskriminierend, sondern weisen den viel besungenen Skill-Bias auf. Dennoch wirkt sich die Einführung von neuen Prozessen oder Produkten nachteilig für Ältere aus.

Mit Querschnittsdaten der Jahre 1993 bis 1995 des IAB-Betriebspanels arbeitet Beckmann (2005). Er schätzt den Anteil Älterer im Betrieb und ermittelt, dass eine vorangegangene technische Änderung die Nachfrage nach Älteren signifikant senkt. Auch Schøne (2009), der die Auswirkungen neuer Technologien auf die betriebliche Altersstruktur anhand norwegischer Daten untersucht, kommt in der Analyse von Querschnitten zu diesem Ergebnis. Bei der Untersuchung von Verlaufsdaten und nach Kontrolle unbeobachteter betrieblicher Heterogenität verschwindet dieser altersverzerrende Effekt, sodass der Autor diesen nicht für kausal hält.

Zusammenfassend sind die Ergebnisse, allerdings auch Konzepte von technischem Wandel und seiner Messung uneinheitlich. Während US-amerikanische Arbeiten mehrheitlich zu einem langfristig austrittsverschiebenden Effekt neigen, kommen die Studien über Deutschland zu dem Ergebnis, dass insbesondere die Einführung von Computern die Erwerbsposition der Älteren im Betrieb nicht verbessert.

\section{A 1.3 Zusammenfassung: Mögliche Bestimmungsgründe für Erwerbs- austritt}

Die folgende Übersicht die beschriebenen Erklärungsansätze für das individuelle Austrittsverhalten fasst das bisher Beschriebene zusammen. Die erwartete Wirkungsrichtung richtet sich dabei an der beschriebenen theoretischen und empirischen Literatur. Weiter unten soll die 
Tabelle als Vorlage zur Beurteilung der Schätzergebnisse diene: Sind die gefundenen empirischen Zusammenhänge im Einklang mit den oben beschriebenen Theorien? Lassen sich die Ergebnisse in Einklang bringen mit den empirischen Ergebnissen der genannten Vorgängerarbeiten?

In den folgenden Unterkapiteln werden zeigen, ob sich die oben präsentierten Ergebnisse empirisch bestätigen lassen. Zunächst wird die verwendete Datenbasis vorgestellt, ihre Vor- und Nachteile diskutiert und Lösungen für Unzulänglichkeiten des Datensatzes präsentiert. Danach folgen deskriptive und mikroökonometrische Ergebnisse.

Tab. 4: $\quad$ Zusammenfassung erwarteter Wirkungen auf Erwerbsaustritt

\begin{tabular}{|c|c|c|}
\hline Angebotsseitige Gründe & Erwartete Wirkung auf die Austrit & tsentscheidung \\
\hline Monetäre Einflüsse & $\begin{array}{l}\text { Hoher Lohn } \\
\text { Hohes Vermögen } \\
\text { Zugangsmöglichkeit zu Frührente }\end{array}$ & $\begin{array}{l}\text { verzögernd } \\
\text { beschleunigend } \\
\text { beschleunigend }\end{array}$ \\
\hline Familiärer Kontext & Erwerbstätigkeit des Partners: & verzögernd \\
\hline Gesundheit & $\begin{array}{l}\text { Kurzfristige Verschlechterung } \\
\text { Andauernde Verschlechterung }\end{array}$ & $\begin{array}{l}\text { beschleunigend } \\
\text { beschleunigend }\end{array}$ \\
\hline \multicolumn{3}{|l|}{ Nachfrageseitige Gründe } \\
\hline Altersstruktur & $\begin{array}{l}\text { Hoher Anteil Jüngerer } \\
\text { Hoher Anteil Älterer }\end{array}$ & $\begin{array}{l}\text { beschleunigend } \\
\text { verzögernd }\end{array}$ \\
\hline Anpassungskosten & Hoher Anteil Randbelegschaft & verzögernd \\
\hline Jobcharakteristika & Physische Anstrengung & beschleunigend \\
\hline Institutionen & $\begin{array}{l}\text { Mitbestimmung } \\
\text { Tarifbindung } \\
\text { Senioritätsentlohnung } \\
\text { Altersdiskriminierung }\end{array}$ & $\begin{array}{l}\text { unbestimmt } \\
\text { eher verzögernd } \\
\text { unbestimmt } \\
\text { unbestimmt }\end{array}$ \\
\hline Technischer Wandel & $\begin{array}{l}\text { Kurzfristige Wirkung } \\
\text { Langfristiger Wirkung } \\
\text { IKT }\end{array}$ & $\begin{array}{l}\text { beschleunigend } \\
\text { verzögernd } \\
\text { neutral }\end{array}$ \\
\hline
\end{tabular}

Quelle: eigene Erstellung 


\section{A 2 Datengrundlage Erwerbsaustritt: Linked Employer Employee Datensatz}

Um die aufgeworfenen Fragestellungen einer empirischen Überprüfung zuzuführen, stehen der Wissenschaft in Deutschland seit wenigen Jahren personenbezogene Mikrodaten zur Verfügung, die gleichzeitig eine Auswertung des betrieblichen Verhaltens erlauben. Im LIAB sind die Erhebungsdaten des IAB-Betriebspanels verknüpft mit prozessgenerierten Personendaten, die sich zum einen aus den Pflichtangaben des Arbeitgebers bei sozialversicherungspflichtiger Beschäftigung eines Arbeitnehmers ergeben (Beschäftigten-Historik-Datei, $\mathrm{BeH}$ ), zum anderen aus den Geschäftsstatistiken der BA (Leistungsempfänger-Historik-Datei, LeH). Die gemeinsam aufbereiteten Datensätze bilden die Beschäftigten-LeistungsempfängerHistorik-Datei (BLH). Nach der Einteilung von Abowd und Kramarz (1999) handelt es sich beim LIAB daher um einen Datensatz, der repräsentativ für Betriebe in Deutschland und ihre Beschäftigten ist und sowohl aus Erhebungs- wie administrativen Daten besteht.

Bei der BeH handelt es sich um Daten, die mindestens jährlich an die Sozialversicherer gemeldet werden. So lässt sich aus den Daten der Beschäftigungsverlauf seit 1975 zurückverfolgen. Aus der LeH ergeben sich darüber hinaus Angaben über Zeiträume, in denen eine Person Leistungen von der BA erhalten hat.

Der prozessgenerierte Charakter der Daten birgt für die Auswertung Vor- und Nachteile: Zwar bürgt die strafbewehrte Verpflichtung des Arbeitgebers zur Meldung einer Beschäftigung an die Sozialversicherer für eine hohe Reliabilität der Daten und schließt ein Hauptproblem von survey-basierten Erhebungen, Rücklauf und Selbstselektion der Teilnahme, aus. Es ergeben sich aus dem Meldeverfahren aber andere Probleme. So werden in den Personendaten systematisch nicht alle abhängig Beschäftigten erfasst und nicht versicherungsrelevante Informationen nur in eingeschränkter Qualität.

Eine Einschränkung ergibt sich aus der Beitragsbemessungsgrenze: Mit dieser Grenze ist gesetzlich die Bemessungsgrundlage für die Beiträge zur Rentenversicherung (Ende 2007: max. 46.800 Euro jährlich) geregelt. Angaben zu Personen, die diesen Betrag überschritten haben, sind in den Daten der BA nicht vorhanden, soweit sie nicht freiwillig pflichtversichert waren. Im letzten Fall sind die Daten zum Tagesentgelt an der Bemessungsgrenze gestutzt. Darüber hinaus wurden Personen mit geringfügiger Beschäftigung erst ab dem Jahr 1999 sozialversicherungspflichtig und konnten in den Jahren davor im vorliegenden Datensatz nicht beobachtet werden. Weitere Ausnahmegruppen bilden Beamte und Personen, die im Ausland beschäftigt sind. Die Auswertungen beschränken sich daher auf der Personenseite auf die Gruppe der Pflicht- und freiwillig Pflichtversicherten. Eine umfassende Datensatzbeschreibung des LIAB findet sich bei Alda und Herrlinger (2005a) S. 8f.. Auf der Betriebsseite steht das IABBetriebspanel, eine jährliche Befragung von Betrieben, die seit 1993 in Westdeutschland und seit 1996 auch in der ehemaligen DDR durchgeführt wird. 
Abb. 5: Schema der Datenaufbereitung

Beschäftigten-Leistungsempfänger-Historik:

Spells seit 1995, Jahrgänge 1945 und früher

$\longrightarrow$

\begin{tabular}{c}
$\begin{array}{c}\text { Personendaten } \\
\text { in Spellform } \\
\mathrm{n}=7.882 .333\end{array}$ \\
\hline
\end{tabular}

Imputation von Lohnangaben an der Bemessungsgrenze

\section{Beschäftigten-Leistungs- empfänger-Historik}

- aktuellem Versicherungsverlauf
Angaben zur Historie 1975 bis 1995 :

- Versicherungspflichtiges Einkommen

- Anzahl der Freistellungsspells

Erstellen von Variablen zu:

- Austrittsereignis

- aktuellem Gesundheitszustand

$$
\downarrow
$$

Reduzierung auf jeweils letzten svpflichtigen Spell im Jahr

\section{Personen-Jahrespanel}

Hinzuspielen der Betriebsvariablen, Erstellen von Ad-hoc-Lösungen für nicht wiederkehrenden Variablen

Auswahl der Personen: x Jahre im BP erfasst
Betriebspanel:
Wellen 1996 bis 2004

Auswahl Personendaten: Aktu-

ell Beschäftigte von BP-

Betrieben, die mindestens zwei

Mal zwischen 1996 und 2004

am Panel teilgenommen haben

- - - - - - - - - - - - 
Auch ist die Zusammenführung der Daten nicht problemlos. Zum einen fanden sich nicht zu jedem Panelbetrieb Personenangaben in den prozessgenerierten Daten, zum anderen waren Abweichungen in der Zahl der Beschäftigten - wie sie sich in aus den Personendaten errechnen - zu den Angaben der Betriebe feststellen. ${ }^{41}$

Für die Wissenschaft stehen standardmäßig zwei Datensätze im Längsformat zur Verfügung. In einer Forschungskooperation mit dem IAB wurde für diese Auswertungen ein eigens auf die Anforderungen dieses Forschungsvorhabens zugeschnittener Datensatz aus den Prozessdaten generiert, der im Folgenden beschrieben wird. ${ }^{42}$

\section{A 2.1 Aufbau des Datensatzes}

Gegenstand der Untersuchung ist der Erwerbsaustritt von älteren Beschäftigten. Angestrebt wurde daher ein Personendatensatz, der sowohl relevante betriebliche wie individuelle Informationen enthält. Ausgehend von den Personendaten der BLH wurde ein Datensatz erstellt, der den besonderen Anforderungen des Auswertungsziels Genüge trägt. Eine schematische Übersicht über die einzelnen Bearbeitungsschritte findet sich in Abb. 5.

\section{A 2.1.1 Personendaten}

Zunächst mussten diejenigen Personen identifiziert werden, für die Betriebsinformationen aus dem IAB-Panel zur Verfügung standen. Deshalb wurden Panelbetriebe ausgewählt, die an mindestens zwei Wellen teilgenommen haben. Ausgehend von diesen Betrieben wurden aus der BLH all diejenigen Personen gezogen, die an mindestens einem der Befragungstermine, dem 30. Juni des Betriebspaneljahres, sozialversicherungspflichtig in dem Betrieb beschäftigt waren.

Um die für den Beobachtungszeitraum rentenrelevanten Jahrgänge zu untersuchen, wurden alle Personen mit einem Geburtsdatum von 1945 und früher ausgewählt. Zudem wurden Personengruppen ausgeschlossen, für die berufsspezifische Zugangsregeln zur Rente gegolten haben konnten. Daher wurden anhand des Personengruppenschlüssels Landwirte und Seeleute ausgesondert. Aus demselben Grund blieben Personen unberücksichtigt, die in die Künstlersozialkasse einzahlen.

Von diesen Personen wiederum wurden die Meldungen aus den Jahren 1996 bis zum aktuellen Rand einschließlich Ende 2004 ausgewählt. Die Beschränkung ergibt sich aus zweierlei Gründen. Im Jahr 1996 begann die Erhebung von Betrieben in der ehemaligen DDR, sodass eine Auswertung früherer Daten nicht sinnvoll erschien. Zudem traten im Jahr 1996 die ersten

\footnotetext{
${ }^{41}$ Vgl. Alda (2005) S. 3ff.

${ }^{42}$ Die Erstellung des Datensatzes wurde vorgenommen durch Mitarbeiter des Forschungsdatenzentrums der BA im IAB. Insbesondere ist dabei Holger Alda zu danken, der auch bei der weiteren Aufbereitung des Datensatzes von unverzichtbarer Hilfe war.
} 
Einschränkungen für den vorzeitigen Renteneintritt in Kraft. Aufgrund der Altersauswahl waren die jüngsten Personen im ersten Beobachtungsjahr 50 Jahre alt, im Jahr 2003, dem letzten Jahr, in dem Austritte beobachtet werden konnten, vollendeten die beobachteten Personen mindestens ihr 58. Lebensjahr. Nach dieser Auswahl betrug der Datensatz 7898140 Meldungen.

Um den Datensatz in eine handhabbare Struktur zu bringen, wurde dieser von einer Spellform in ein Jahrespanel umgeformt. Dabei wurde jeweils die letzte sozialversicherungspflichtige Meldung einer Person und eines Jahres beibehalten. Durch die notwendige Beschränkung auf Spells in sozialversicherungspflichtiger Beschäftigung konnten auch Inkonsistenzen vermieden werden, wie sie bei der Arbeit mit prozessgenerierten Daten wie der Integrierten Erwerbsbiografie anfallen. Unlogische Überschneidungen ${ }^{43}$ von Beschäftigungs- und Leistungsmeldungen mussten hier nicht näher beachtet werden. Bei gleichzeitigem Ende von mehreren parallelen Beschäftigungszeiträumen wurde die Hauptbeschäftigung als einzige beibehalten.

\section{A 2.1.2 Betriebsdaten}

Im nächsten Schritt wurden die Personendaten um betriebliche Informationen aus dem IABBetriebspanel ergänzt. Über eine sowohl in beiden Datensätzen enthaltenen eindeutigen Betriebsnummer konnten so den einzelnen Beschäftigungsspells Betriebe zugeordnet werden, sofern sie in dem entsprechenden Jahr am Panel teilgenommen hatten. Durch die oben beschriebene Auswahl der Personen wurde sichergestellt, dass sich jeder Person mindestens in einem Jahr entsprechende Betriebsdaten zuordnen ließen. Die folgende Grafik (vgl. Abb. 6) soll einen Eindruck darüber vermitteln, über welchen Zeitraum Personen mitsamt den Betriebsdaten beobachtet werden konnten. Daraus wird ersichtlich, dass für eine Vielzahl der Personen nur recht kurze Zeiträume von sozialversicherungspflichtiger Beschäftigung in den Datensatz eingingen. Gründe dafür sind neben dem individuellen Ende der Erwerbstätigkeit das in den deskriptiven Statistiken beschrieben wird - ein Ausscheiden der beschäftigenden Betriebe aus dem Panel oder ein Wechsel der Person in einen anderen, nicht befragten Betrieb. Allerdings sind für die Anwendung der folgenden ökonometrischen Verfahren keine bis zum beobachteten Austritt vollständigen Datensätze notwendig. Da die Personendaten nicht an das Vorhandensein von Betriebsinformationen gebunden sind, konnte ein Verbleib in einer Beschäftigung beobachtet werden. Die Person geht dann bis zu diesem Zeitpunkt als zensiert in den Datensatz ein. Auch Unter-brechungen in der Beobachtung von Betriebsdaten, die durch Betriebswechsel in einen anderen Panelbetrieb oder durch erneute Teilnahme des Betriebs am Panel entstehen konnten, stellen keinen Ausschlussgrund aus dem Datensatz dar.

\footnotetext{
${ }^{43}$ Eine Dokumentation der Probleme mit den Daten der IEBS und ihrer Lösungsmöglichkeiten bieten Jaenichen et al. (2005).
} 
Abb. 6:

Anzahl der Spells nach Jahren der Betriebszugehörigkeit im aktuellen Panelbetrieb

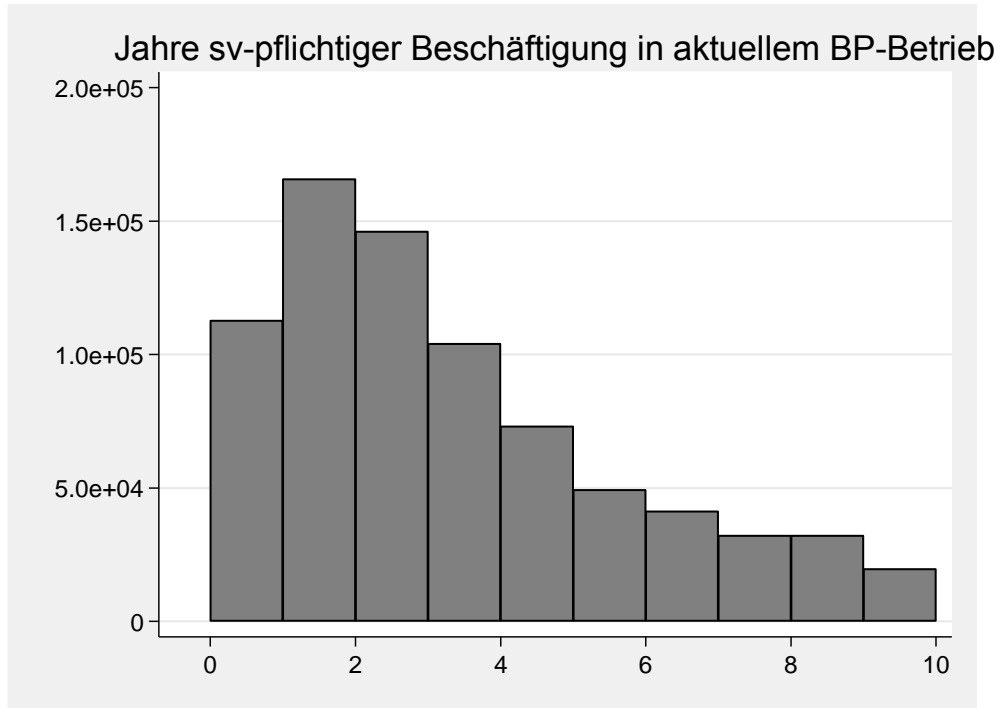

Quelle: LIAB, eigene Berechnungen

Bei der Interpretation der Ergebnisse muss beachtet werden, dass es sich bei den Daten des Panels meist um stichtagsbezogene Angaben handelt, die sich auf den Zeitpunkt 30. Juni des aktuellen Jahres bezogen. Auch konnten nur all diejenigen Betriebsvariablen in den Datensatz einfließen, die durchgängig von 1996 bis 2004 erhoben wurden. Daneben wurde aus der gesamten BLH die Altersstruktur des Betriebes hinzugespielt.

\section{A 2.2 Variablenauswahl und -aufbereitung}

Um den so in seinen Beobachtungen eingegrenzten Datensatz auszuwerten, mussten die Variablen in eine angemessene Form gebracht werden. Nicht alle der in den Personen- und Betriebsdaten enthaltenen Merkmale liegen vor, wie es für die Auswertungszwecke wünschenswert wäre. Im Folgenden soll daher dokumentiert werden, wie die Variablen für die empirische Analyse ausgewählt und aufbereitet wurden.

\section{A 2.2.1 Personendaten}

Die BLH auf der Personenseite wurde generiert aus den Historik-Dateien zur Beschäftigung und zum Leistungsbezug. Die erfassten Merkmale der beiden Datensätze sind allerdings nicht identisch: Beide Datenquellen enthalten neben den notwendigen Angaben zu Inhalt, Anfang und Ende eine Spells auch Informationen zum Geschlecht, dem Alter und der Nationalität einer Person. Angaben, die ausschließlich in den LeH-Daten zu finden waren, mussten unberücksichtigt bleiben, da sich sonst der Datensatz auf die Gruppe derjenigen beschränkt hätte, die im Beobachtungszeitraum Unterstützungsleistungen erhalten hatten, und eine Verzerrung der Ergebnisse durch eine hochselektive Stichprobenziehung nicht zu vermeiden gewesen wären. 


\section{A 2.2.1.1 Austritt aus dem Erwerbsleben}

Die für diese Studie zunächst geplante Angabe des Renteneintritts ist im Datensatz nicht unmittelbar erfasst. Wann eine Person in die Rente eintritt, ist den Angaben nicht direkt zu entnehmen, sodass auf das Ende des Erwerbslebens ausgewichen werden musste. Allerdings stehen die beiden Ereignisse Rentenein- und Erwerbsaustritt in einem auch zeitlich engen $\mathrm{Zu}$ sammenhang, der sich für vorgezogene Renten schon aus den Beschränkungen des Hinzuverdienstes ergibt. Wübbeke (2005b, S. 47f.) zeigt für die Jahrgänge 1920 bis 1930, dass für die Mehrheit der Versicherten zwischen Beschäftigungsaustritt und Renteneintritt höchstens 31 Tage lagen. Auch der Anteil derer, die parallel zum Rentenbezug noch sozialversicherungspflichtig beschäftigt war, lag nach Wübbekes Berechnungen je nach Kohorte bei rd. zehn Prozent für Männer und gut fünf Prozent für Frauen. ${ }^{44}$

Leider sind auch die Abmeldegründe aus der sozialversicherungspflichtigen Beschäftigung, zu denen auch der Eintritt in die Altersrente gehört, nur sehr unvollständig erfasst, so dass der Austritt aus dem Erwerbsleben nur annähernd ermittelt werden konnte. Dazu wurde als Austrittsereignis das Ende des letzten beobachteten Beschäftigungsspells definiert. Um Fehldeutungen zu vermeiden, wurde am Ende des Beobachtungszeitraums das Ereignis Beschäftigungsaustritt nur dann definiert, wenn danach mindestens ein Jahr lang keine neue Beschäftigung beobachtet werden konnte. ${ }^{45}$ De facto endet der Beobachtungszeitraum damit ein Jahr vor der letzten Beschäftigungsmeldung, da nach dieser Definition in den letzten zwölf Monaten keine Austritte mehr verzeichnet werden konnten. Das Vorgehen erscheint vertretbar, da die betroffenen Personen zum fraglichen Zeitpunkt schon ein Alter von mindestens 58 Jahren erreicht hatten und ist bekannt, dass die Wiedereinstellungschancen von Entlassenen in fortgeschrittenem Alter gering sind (vgl. z.B. Chan und Stevens (2001)).

\section{A 2.2.1.2 Geschlecht/Region}

Für Männer und Frauen, sowie Ost- und Westdeutsche waren strukturell unterschiedliche Ergebnisse zu erwarten. Nicht nur Unterschiede in den typischen Erwerbsbiografien, sondern auch die erwähnten unterschiedlichen Rentenzugangsformen für Männer und Frauen lassen diese getrennte Behandlung sinnvoll erscheinen. Auch die Datenlage spricht für ein solches

\footnotetext{
${ }^{44}$ Frühere Untersuchungen zum Erwerbsverhalten von Rentnern zeigen ebenfalls eine geringe Erwerbstätigkeit von Rentnern bzw. Personen im Rentenalter: Helberger und Schwarze (1986) kommen auf einen Anteil von 3,6\%, Pischner und Wagner (1992) auf 5,2\%, Wachtler et al. (1995) auf 8,7\%. Allerdings machen Büttner et al. (2005) darauf aufmerksam, dass sich die zeitliche Lücke zwischen Erwerbsaus- und Renteneintritt in den vergangenen Jahren ausgeweitet hat.

${ }^{45}$ Einen Spezialfall stellt die Altersteilzeit dar. Zwar lassen sich aus der Variablen „Personengruppe“ auch die Personen in Altersteilzeit erkennen (vgl. Alda und Herrlinger (2005a und 2005c). Es wurde jedoch darauf verzichtet, dies als Anzeichen für einen Austritt aus dem Erwerbsleben zu interpretieren. Personen sind nach dem ATG verpflichtet, nach Ablaufen der Altersteilzeit die Rente anzutreten und müssten daher nach der gewählten Methode bis Ende 2003 erfasst sein. Nur im Jahr 2004 hätte das Ende der Altersteilzeit dem Datensatz zusätzliche Informationen zuführen können, eine Berücksichtigung allerdings zu einer Inkonsistenz der OutcomeVariablen geführt, da in diesem Jahr nur Austritte nach Altersteilzeit beobachtet worden wären.
} 
Vorgehen, sind doch für Ostdeutschland betriebliche Angaben erst ab dem Jahr 1996 und verlässliche Daten zum Erwerbsverlauf erst ab 1993 verfügbar. Die beiden Angaben zu Geschlecht und Region waren daher nur zu Datenaufbereitungszwecken notwendig. Dabei richtet sich die regionale Zuordnung nach dem Wohnort.

\section{A 2.2.1.3 Monetäre Merkmale}

Einzelne Angaben sind in den Personendaten nicht in dem Umfang enthalten, wie es für die Auswertung wünschenswert gewesen wäre. So spiegelt sich z.B. in der Einkommensvariable, die im Datensatz als sozialversicherungspflichtiges Bruttotagesentgelt vorkommt, der prozessgenerierte Charakter der Personendaten wider: ${ }^{46}$ Für niedrige Einkommensbereiche ergibt sich eine linksseitige Stutzung aus der Tatsache, dass geringfügig Beschäftigte bis einschließlich 1998 nicht der Pflicht zu Abgaben zu den Systemen der Sozialversicherung unterlagen. Solche Beschäftigungsverhältnisse tauchen in den Daten auch nicht auf. Beobachtungen mit geringfügiger Beschäftigung wurden daher auch nach 1998 aus dem Datensatz entfernt, um die Einheitlichkeit zu gewährleisten. Für die höheren Arbeitseinkommen führt die Beitragsbemessungsgrenze zu einer Rechtszensierung der Variablen. Sie besteht für den gesamten Beobachtungszeitraum, variiert allerdings mindestens jährlich und unterscheidet sich nach Ost und West, wobei der Standort des Betriebes maßgeblich ist. ${ }^{4748}$

Die Angaben zur Einkommenshöhe ab der Bemessungsgrenze wurden daher in Anlehnung an das Stata-ado-File von Gartner (2005) imputiert. Wegen der zeitlichen und regionalen Variation der Beitragsbemessungsgrenzen wurden getrennt nach den beiden Landesteilen, für jedes Jahr und Geschlecht insgesamt 36 verschiedene Imputationen durchgeführt, die jeweils auf einer Tobit-Regression des Tagesentgeltes auf die Variablen Alter (auch seine Polynome), berufliche Stellung, Berufsgruppe (als Einsteller), Branche (ebenfalls als Einsteller) und einen Nationalitätsdummy beruhten. Da eine genaue Angabe der geleisteten Stundenzahl pro Tag in den Daten fehlt, kann der Stundenlohn nicht erfasst werden.

\section{A 2.2.1.4 Gesundheit}

Wie oben beschrieben spielt der Gesundheitszustand einer Person eine wichtige Rolle bei der Schätzung der Erwerbsaustrittsentscheidung. Eine direkte Angabe zum Gesundheitszustand fehlt allerdings in den Daten. Dieses Problem kann jedoch gemildert werden, wenn Zeiten, in denen ein Beschäftigungsverhältnis bestand, aber kein Entgelt bezahlt wurde, als Proxy für den gesundheitlichen Zustand herangezogen werden. Hintergrund für diese Annäherung ist

\footnotetext{
${ }^{46}$ Wie alle anderen monetären Variablen sind die Werte für den gesamten Zeitraum in Euro umgerechnet.

${ }^{47}$ Eine Auflistung der jeweils in Ost- und Westdeutschland gültigen Geringfügigkeits- und Beitragsbemessungsgrenzen findet sich bei Alda und Herrlinger (2005a) S. 58.

48 Gelegentlich kommt es zu Angaben, die die aktuelle und für den Landesteil maßgebliche Beitragsbemessungsgrundlage überschreiten. Alda und Herrlinger (2005a) verweisen jedoch darauf, dass es sich dabei um einmalige Sonderzahlungen handelt.
} 
das Entgeltfortzahlungsgesetz (EntgFG). Nach $\S 3$ hat der Arbeitnehmer im Krankheitsfall zwar Anspruch auf Entgeltfortzahlung. Der Anspruch endet aber nach den ersten sechs Wochen der krankheitsbedingten Arbeitsunfähigkeit. Danach greift SGB V § 44 mit der Zahlung von Krankengeld. ${ }^{49}$

Angesichts der langen Dauer von sechs Wochen bis zur Zahlung des Krankengeldes kann man davon ausgehen, dass es sich bei den Freistellungsspells um ernsthafte Beeinträchtigungen der Arbeitsfähigkeit handelt.

Diese Beobachtung geht in zweifacher Weise in den neuen Datensatz ein: als jeweils aktuell kumulierte Anzahl solcher Freistellungsspells seit 1975 (bzw. seit 1990 für Ostdeutschland) und als Dummy, der angibt, ob in den jeweils letzten zwei Jahren ein solches Ereignis zu beobachten war. Mit der ersten Variablen wurde eine Variable generiert, die den allgemeinen Gesundheitszustand annähern soll. In der zweiten Variablen sollen aktuelle Veränderungen des Gesundheitszustandes abgebildet werden. Auch die Altersvariable kann als zusätzliche Kontrolle für den gesundheitlichen Zustand interpretiert werden.

\section{A 2.2.1.5 Familiärer Kontext}

Leider ist der familiäre Kontext nur sehr bedingt in den Daten erfasst. So ist nur in den Leistungsempfänger-Daten der Familienstand des Versicherten angegeben. Selbst in den LeHDaten ist aber kein Bezug zu einem Ehepartner beobachtbar, sodass selbst für die Subpopulation derer, für die ein Leistungsspell beobachtet wurde, das Phänomen simultaner Ruhestandsentscheidung von Ehepartnern nicht empirisch modelliert werden kann.

Die Angabe zu Kindern ist ebenfalls nur in der LeH verzeichnet. Selbst dort ist sie nur als Dummy-Variable beobachtbar. Zwar ließe sich das Alter der Kinder über die erste Beobachtung einer entsprechenden Dummyausprägung annähern, ihre Anzahl ist den Daten aber nicht zu entnehmen. Eine Aufnahme der Variablen erschien daher nicht ratsam.

\section{A 2.2.1.6 Ausbildung}

Die Angaben zur Ausbildung sind ebenfalls in den Beschäftigungsmeldungen enthalten. So wird vom Arbeitgeber in den „Angaben zur Tätigkeit“ neben Beruf und beruflicher Stellung auch der so genannte B2-Schlüssel übermittelt. Die daraus gebildete Variable weist in zweier-

\footnotetext{
${ }^{49}$ Zeiten ohne Entgelt trotz eines bestehenden Arbeitsverhältnisses müssen nicht nur aus Gründen der eigenen Gesundheit entstehen. Andere Beispiele sind Regelungen des Mutterschutzgesetzes (MSchG, § 3,1), das die Schutzzeiten von Müttern unmittelbar vor und nach der Entbindung regelt, und das Arbeitsplatzschutzgesetz (ArbPISchG), das den Fortbestand des Arbeitsverhältnisses bei Einberufung zu Wehr- oder Zivildienst regelt. Ebenfalls könnten solche Zeiträume beobachtet werden für Personen, die nach $\S 15$ des Gesetzes zum Erziehungsgeld und zur Elternzeit (BErzGG) Anspruch auf Elternzeit haben. In allen Fällen ist der Arbeitgeber nicht zu einer Entgeltzahlung verpflichtet; das Beschäftigungsverhältnis besteht aber weiter. Allerdings ist nicht zu erwarten, dass die angeführten Beispiele angesichts der Altersbeschränkung der untersuchten Personen häufig auftreten. Einzige Ausnahme stellen für die kumulierte Anzahl an solchen Spells seit 1975 die jüngeren Frauen in Westdeutschland dar.
} 
lei Hinsicht Unzulänglichkeiten auf. Einerseits enthält sie kombinierte Angaben zum höchsten Abschluss in Schul- und Berufsausbildung. Die Kategorien vereinen die Angabe des höchsten Schulabschlusses mit der Information über die Berufsausbildung. Daneben gibt es die Ausprägungen „Hochschulabschluss“ und „Fachhochschulabschluss“, die nicht mit einer (weiteren) Berufsausbildungsangabe angereichert sind. Schulische und berufliche Ausbildung konnten daher nicht separat als Einflussgrößen ausgewertet werden.

Andererseits weisen Meinken und Koch (2004) darauf hin, dass die Ausbildungs-Daten möglicherweise von mangelnder Qualität sind, da es sich nicht um ein versicherungsrelevantes Merkmal handelt. Dies drückt sich in einer - besonders für geringfügig Entlohnte - hohen Zahl von fehlenden Werten aus (vgl. Alda und Herrlinger (2005b) S. 4). Es lässt sich aber auch daran erkennen, dass die Werte sich oft erst dann ändern, wenn ein Arbeitgeberwechsel stattgefunden hat und nachträglich erworbene Qualifikationen nachgemeldet werden. Für die hier verwendete Teilstichprobe der Älteren dürfte insbesondere der erste Aspekt mangelnder Datenqualität von Bedeutung sein.

Fitzenberger et al. (2005) stellen einige Imputationsverfahren vor, mit deren Hilfe die oben genannten Schwierigkeiten behoben werden sollen. Allerdings ergaben sich für die imputierten Variablen in dem angeführten Beispiel einer Lohnregression keine gravierenden Änderungen der geschätzten Parameter, weshalb in der vorliegenden Arbeit von einer Imputation der Variablen Abstand genommen wurde.

\section{A 2.2.1.7 Berufliche Stellung}

Die berufliche Stellung ergibt sich ebenfalls aus den „Angaben zur Tätigkeit“ aus den Beschäftigungsmeldungen. Die Variable ist daher mit ähnlichen Problemen behaftet wie die Ausbildungsvariable. Zwar sind fehlen die Angaben wesentlich seltener als diese, doch ist davon auszugehen, dass eine regelmäßige Überprüfung der beruflichen Stellung bei Personen mit dauerhafter Beschäftigung bei ein und demselben Arbeitgeber nicht stattfindet.

Auch bei diesem Merkmal wurden Aspekte der Tätigkeit vermischt. Neben den möglichen Ausprägungen „Auszubildende“, „ohne formale Qualifikation“, „Facharbeiter“, „Meister/Polier“ und „Angestellter“ sind Teilzeitbeschäftigte gesondert und unabhängig von ihrer beruflichen Stellung erfasst. Die Zuordnung in die Kategorien „Arbeiter“ und „Angestellter“ richtete sich dabei ausschließlich nach der Art der Rentenversicherungspflicht (vgl. Alda und Herrlinger (2005a)).

\section{A 2.2.1.8 Historik-Daten}

Neben den aktuellen Daten der jeweiligen Spells, wurden aus der für Westdeutschland bis ins Jahr 1975 zurückreichenden Historik der BLH Variablen generiert, die den Erwerbsverlauf widerspiegeln sollen. Um einen näherungsweisen Wert für die Rentenanwartschaften der Personen zu erhalten, wurde die Summe der sozialversicherungspflichtigen Einkommen der Per- 
sonen seit dem Beginn der Erfassung bis zum jeweiligen Spell erstellt. Da dieser Wert ohnehin nur eine grobe Approximation der tatsächlichen Anwartschaften darstellt, wurde auf eine Gewichtung der Einzelbeträge verzichtet. Auch die Anzahl der bis zu diesem Zeitpunkt beobachteten Freistellungsspells wurde jeweils vom Beginn der Erfassung an aufsummiert.

Um die Akkumulation von betriebsspezifischem Humankapital zu kontrollieren, wurde eine Variable erstellt, die den Eintritt in den Betrieb abbildet. Darüber hinaus soll eine Variable, die den Eintritt in das Erwerbsleben darstellt, allgemeines Humankapital repräsentieren. Allerdings sind alle diese Angaben erst ab dem Jahr 1975 für Westdeutschland und dem Jahr 1990 für Ostdeutschland bzw. ab dem Beginn der ersten sozialversicherungspflichtigen Beschäftigung vorhanden und daher zensiert. Zu beachten ist, dass die Angaben unabhängig von der Teilnahme des Betriebes am IAB-Betriebspanel erfolgen.

\section{A 2.2.2 Betriebsdaten}

Um ein Mindestmaß an Betriebsinformation in den einzelnen Beschäftigungsbiografien zu gewährleisten, wurden für diese Arbeit nur diejenigen Betriebe ausgewählt, die seit 1996 an mindestens zwei Wellen des IAB-Betriebspanels teilgenommen hatten. Im Panel erhobene Merkmale konnten in dieser Längsschnitt-Analyse einbezogen werden, wenn sie durchgängig in jeder Welle des Analysezeitraums erfragt wurden. Leider genügen einige der Variablen nicht dieser Bedingung. Tab. 5 gibt einen Überblick über die Verfügbarkeit von für die Erwerbsbeteiligung Älterer bedeutsamen Variablen im Panelbverlauf. Dunkel eingefärbte Jahreszellen bedeuten, dass die Variable im entsprechenden Jahr nicht in einer für die Verlaufsanalyse notwendigen Weise erhoben wurde. Helle Zellen stellen dagegen auswertbare Daten im jeweiligen Jahr dar.

Eine denkbare Imputation von Werten aus den Vorgänger- oder Nachfolgejahren erschien in nur wenigen Fällen sinnvoll. Sie ist zum einen mit einem Informationsverlust der entsprechenden Variablen verbunden. Zum anderen wird sie in der folgenden Ereignisanalyse mit einem Verlust an Beobachtungen erkauft, da die Substitution mit Werten aus anderen Erhebungsjahren nur dann erfolgreich ist, wenn der Betrieb in diesen auch teilgenommen hat.

$\mathrm{Zu}$ beachten ist weiterhin, dass die Angaben aus dem IAB-Betriebspanel zeitlich nicht genau mit den Individualdaten auf der Personenebene übereinstimmen müssen. Während es sich bei den betrieblichen Informationen oft um zeitpunktbezogene Daten handelt, die jeweils zum 30 . Juni ihre Gültigkeit haben, sind die Personenangaben zeitraumbezogen. Kleinere zeitliche Abweichungen sind daher für einige Variablen unvermeidbar.

In den Datensatz konnten all diejenigen Betriebsvariablen einfließen, die durchgängig von 1996 bis 2004 erhoben wurden. Daneben wurde aus den Personendaten der gesamten BLH die Altersstruktur des Betriebes hinzu gespielt. 
Tab. 5:

Kontinuität von Variablen im Verlauf des Betriebspanels 1996 bis 2004

\begin{tabular}{|c|c|c|c|c|c|c|c|c|c|c|}
\hline Betriebsvariablen & & 1996 & 1997 & 1998 & 1999 & 2000 & 2001 & 2002 & 2003 & 2004 \\
\hline \multicolumn{11}{|l|}{ Institutionell } \\
\hline \multirow[t]{2}{*}{ Tarifvertrag } & Branchentarifvertrag & & & & & & & & & \\
\hline & Haus- bzw. Firmentarifvertrag & & & & & & & & & \\
\hline \multirow[t]{2}{*}{ Mitbestimmung } & Betriebsrat/Personalrat & & & & & & & & & \\
\hline & $\begin{array}{l}\text { andere Form der Mitarbeiter- } \\
\text { vertretung }\end{array}$ & & & & & & & & & \\
\hline \multirow[t]{2}{*}{ Betriebsgröße nach KSchG } & $\begin{array}{l}\text { Aufteilung nach wöchentlicher } \\
\text { Arbeitszeit }\end{array}$ & & & & & $\begin{array}{c}\text { Durch } \\
\text { Mittelwert } \\
\text { ersetzt }\end{array}$ & & & & \\
\hline & Auszubildende & & & & & & & & & \\
\hline \multicolumn{11}{|l|}{$\begin{array}{l}\text { Beihilfen } \\
\text { (Angaben bez. auf Vorjahr) }\end{array}$} \\
\hline & Lohnkostenzuschüsse Ältere & & & & & & & & & \\
\hline & Altersteilzeitzuschuss & & & & & & & & & \\
\hline & Eingliederungszuschuss & & & $\begin{array}{l}\text { Eingliede- } \\
\text { rung } \\
\text { allgemein }\end{array}$ & $\begin{array}{l}\text { Eingliede- } \\
\text { rung } \\
\text { allgemein }\end{array}$ & $\begin{array}{l}\text { Einglie- } \\
\text { derung } \\
\text { allgemein }\end{array}$ & $\begin{array}{l}\text { Eingliede- } \\
\text { rung } \\
\text { allgemein }\end{array}$ & $\begin{array}{l}\text { Eingliede- } \\
\text { rung } \\
\text { allgemein }\end{array}$ & $\begin{array}{l}\text { Eingliede- } \\
\text { rung } \\
\text { allgemein }\end{array}$ & \\
\hline & $\begin{array}{l}\text { Anzahl der geförderten Be- } \\
\text { schäftigten }\end{array}$ & & & & & & & & & \\
\hline
\end{tabular}

Quelle: eigene Erstellung

Auswertbare Angaben

Keine/ nicht auswertbare Anga-

ben 
Kontinuität von Variablen im Verlauf des Betriebspanels 1996 bis 2004 - fortgesetzt -

\begin{tabular}{|c|c|c|c|c|c|c|c|c|c|c|}
\hline \multicolumn{2}{|l|}{ Betriebsvariablen } & 1996 & 1997 & 1998 & 1999 & 2000 & 2001 & 2002 & 2003 & 2004 \\
\hline \multicolumn{11}{|l|}{ Anpassungskosten } \\
\hline \multirow[t]{2}{*}{ Geschäftserwartungen } & $\begin{array}{l}\text { Änderung Geschäftsvolu- } \\
\text { men laufendes Jahr }\end{array}$ & & & & & & & & & \\
\hline & $\begin{array}{l}\text { Änderung Geschäftsvolu- } \\
\text { men kommendes Jahr }\end{array}$ & & & & & & & & & \\
\hline \multicolumn{11}{|l|}{ Anzahl Auszubildende } \\
\hline \multirow[t]{3}{*}{ Überstunden } & Dummy & & & & $\begin{array}{l}\text { Angabe Vor- } \\
\text { jahr }\end{array}$ & & $\begin{array}{l}\text { Angabe } \\
\text { Vorjahr }\end{array}$ & $\begin{array}{l}\text { Angabe Vor- } \\
\text { jahr }\end{array}$ & $\begin{array}{l}\text { Angabe Vor- } \\
\text { jahr }\end{array}$ & $\begin{array}{l}\text { Angabe } \\
\text { Vorjahr }\end{array}$ \\
\hline & $\begin{array}{l}\text { Prozent der Beschäftigten } \\
\text { Wie viele Überstunden? }\end{array}$ & & & & $\begin{array}{l}\text { Anteil Besch. } \\
\text { bez. ÜS } \\
\text { Bezahlte ÜS }\end{array}$ & & $\begin{array}{l}\text { Anteil Besch. } \\
\text { bez. ÜS } \\
\text { Bezahlte ÜS }\end{array}$ & $\begin{array}{l}\text { Anteil Besch. } \\
\text { bez. ÜS }\end{array}$ & $\begin{array}{l}\text { Anteil Besch. } \\
\text { bez. ÜS }\end{array}$ & \\
\hline & $\begin{array}{l}\text { Anteil bezahlte Überstun- } \\
\text { den }\end{array}$ & & & & $\begin{array}{l}\text { Bezahlt- Frei- } \\
\text { zeitausgleich - } \\
\text { teils }\end{array}$ & & $\begin{array}{l}\text { Bezahlt- } \\
\text { Freizeitaus- } \\
\text { gleich - teils }\end{array}$ & $\begin{array}{l}\text { Bezahlt- } \\
\text { Freizeitaus- } \\
\text { gleich - teils }\end{array}$ & $\begin{array}{l}\text { Bezahlt- Frei- } \\
\text { zeitausgleich - } \\
\text { teils }\end{array}$ & \\
\hline \multirow[t]{3}{*}{ Befristet Beschäftigte } & Dummy & & & & & & & & & \\
\hline & Anzahl & & & & & & & & & \\
\hline & Anzahl Frauen & & & & & & & & & \\
\hline \multirow[t]{2}{*}{ Aushilfen, Praktikanten } & Dummy & & & & & & & & & \\
\hline & Anzahl & & & & & & & & & \\
\hline \multirow[t]{2}{*}{ Freie Mitarbeiter } & Dummy & & & & & & & & & \\
\hline & Anzahl & & & & & & & & & \\
\hline \multirow[t]{2}{*}{ Leiharbeiter } & Dummy & & & & & & & & & \\
\hline & Anzahl & & & & & & & & & \\
\hline \multirow[t]{4}{*}{ Nachfragesysteme } & Anteil der Beschäftigten & & & & & & & & & \\
\hline & bis 30 Jahren & & & & & & & & & \\
\hline & 31-50 Jahren & & & & & & & & & \\
\hline & 51 Jahre und älter & & & & & & & & & \\
\hline
\end{tabular}


Kontinuität von Variablen im Verlauf des Betriebspanels 1996 bis 2004 - fortgesetzt -

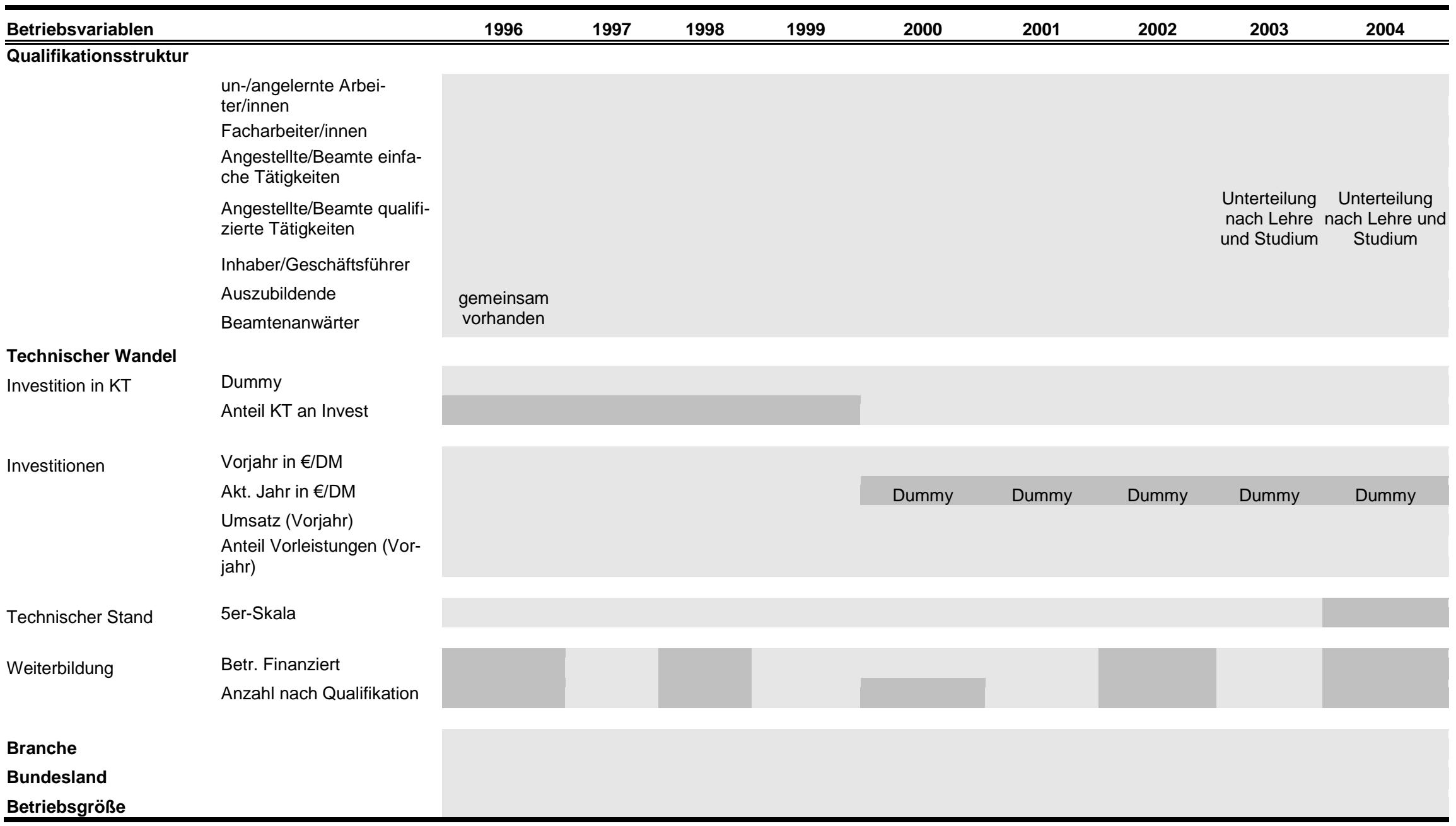




\section{A 2.2.2.1 Institutionen}

Unter den möglichen Variablen, die institutionelle Beschränkungen wiedergeben könnten, konnte die Angabe zur Existenz eines Tarifvertrages einbezogen werden. Die Variable enthält neben der Ausprägung „Branchentarifvertrag“ die Möglichkeit „Haustarifvertrag“ und ist durchgängig einheitlich erhoben.

Zudem konnte die Information, ob ein Betriebs- oder Personalrat vorhanden ist, berücksichtigt werden. Mögliche andere Mitarbeitervertretungen wurden erst seit dem Jahr 2003 in den Fragenkatalog aufgenommen und kamen daher für die Auswertung nicht in Betracht.

Problematisch gestaltete sich die Frage, ob ein Betrieb im jeweiligen Jahr dem Kündigungsschutzgesetz unterliegt. Um dem Gesetz folgend die Anzahl der Beschäftigten zu berechnen, muss neben dem Abzug der Auszubildenden auch die Anzahl der Teilzeitbeschäftigten ermittelt werden. Diese wurde im Jahr 2000 nicht erfragt und daher mit Hilfe von Mittelwerten aus dem vorangegangenen und dem Nachfolgejahr geschätzt. ${ }^{50}$

Auch Informationen über staatliche Beihilfen an den Betrieb konnten aufgrund einer im zeitlichen Verlauf uneinheitlichen Befragung im Betriebspanel nicht in die Analyse mit eingehen. So wurde die Inanspruchnahme von Lohnkostenzuschüssen für Ältere nur in den Jahren 1997 und 1998 erfragt. Von 1998 bis einschließlich 2003 sollten die Betriebe Auskunft über Lohnkostenzuschüsse allgemein Auskunft geben.

\section{A 2.2.2.2 Anpassungskosten}

Die für die Anpassung der Arbeitsnachfrage wohl wichtigste Variable, die der Geschäftserwartungen, ist im Betriebspanel durchgängig erhoben und gibt in Prozent die Änderung des Geschäftsvolumens an, die für das laufende Geschäftsjahr gegenüber dem vergangenen erwartet werden. Der Ausblick auf das folgende Jahr taucht im Fragebogen nur in zwei Jahren auf. Als mögliche Anpassungsformen an eine geänderte Arbeitsnachfrage kann der Anteil der befristet Beschäftigten und der Auszubildenden kontrolliert werden.

Nicht durchgängig erfragt sind dagegen die Überstunden, die sich ebenfalls als Anpassungsmodus bei Nachfrageschwankungen anbieten und damit eine konkurrierende Strategie zur Weiterbeschäftigung von Älteren darstellen. Sie können ebenso wenig in die Auswertung einbezogen werden wie die Anzahl an Aushilfen, Praktikanten, freien Mitarbeitern und Leiharbeitern, da diese in den Jahren 1999 bis 2001 fehlen. Stattdessen steht der Anteil der nicht sozialversicherungspflichtig Beschäftigten zur Verfügung.

\section{A 2.2.2.3 Altersstruktur}

\footnotetext{
${ }^{50}$ Eine genaue Darstellung des Geltungsbereiches des Kündigungsschutzgesetzes und seinen Änderungen im Beobachtungszeitraum findet sich in Kapitel B.
} 
Zusätzlich zu den Daten, die sich direkt dem Betriebspanel entnehmen ließen, konnte aus der BLH die zum 30. Juni des jeweiligen Jahres aktuelle Altersstruktur des Betriebes hinzugespielt werden, anhand derer Substitutionsbeziehungen zwischen einzelnen Altersgruppen und den älteren Beschäftigten abschätzbar sind. Die Altersstruktur geht als Anteil der bis 30Jährigen, der 31- bis 50-Jährigen und der 51-Jährigen und Älteren in den Datensatz ein. Weitere, in der Theorie geläufige Produktionsfaktoren wie der Kapitaleinsatz sind nicht kontrollierbar.

\section{A 2.2.2.4 Qualifikationsstruktur}

Im Betriebspanel durchgängig erhoben sind die Angaben zur Anzahl der ungelernten Arbeiter und Facharbeiter sowie der Angestellten mit einfachen und qualifizierten Tätigkeiten. Sie fließen als Anteile an der Gesamtzahl der Beschäftigten als Kontrollvariablen in die folgenden Verweildaueranalysen ein.

\section{A 2.2.2.5 Technischer Wandel}

Inwieweit der Einzug neuer Technologien in den Betrieb zu einem beschleunigten oder verzögerten Austritt aus dem Erwerbsleben führt, soll anhand von drei Fragen im Betriebspanel untersucht werden. Erstens geht die Angabe ein, ob der Betrieb im jeweils vergangenen Jahr Investitionen in Kommunikationstechnik getätigt hat. Zweitens kann die Angabe zum Stand der technischen Anlagen, die in einer 5er-Skala vorliegt, die längerfristigen Auswirkungen von technischem Fortschritt kontrollieren. Zuletzt dient der Investitionsanteil am Umsatz im Vorjahr als Regressor, der kurzfristige Einflüsse von technischem Wandel auffängt.

Die Frage nach dem Anteil von ITK-Investitionen an den Gesamtinvestitionen wurde erst im Jahr 2000 erstmals erhoben und musste daher unberücksichtigt bleiben. Auch musste von einer Einbeziehung von Angaben aus den Vorjahren, die für einen Vergleich mit der bestehenden Literatur wünschenswert gewesen wären, abgesehen werden, da die Information für neu ins Panel aufgenommene Betriebe nicht beobachtbar gewesen wäre und zu einem Verlust an Spells mit Betriebsinformationen geführt hätte.

Informationen zu Investitionen im aktuellen Jahr konnten ebenso wenig in die Auswertung einfließen wie die Angaben zur betrieblichen Weiterbildung. Die Originalfrage im Panel bezog sich - wenn sie erhoben wurde - jeweils auf das erste Halbjahr. Es war daher nicht möglich, diese Angabe sinnvoll auf andere Jahre zu übertragen, und gleichzeitig eine jährlich einheitliche Interpretation einzuhalten.

\section{A 2.2.2.6 Sonstige betriebliche Variablen}

Zusätzlich zu den hier aufgeführten Variablen geht die Angabe zur Branche in den Datensatz ein. Sie kann als Kontrolle spezifischer konjunktureller Einflüsse und auch unbeobachteter Heterogenität des Gesundheitszustandes dienen. Ausgehend von der Wirtschaftszweigklassifizierung 1993 aus den Personendaten wurde eine Einteilung in 14 Branchen vorgenommen. 
Technische Probleme waren bei der Vereinheitlichung dieser Branchenvariable insofern zu meistern, als die Brancheneinteilung sich im Jahr 2000 ändert. Soweit möglich wurden auf Personenebene die Werte aus dem Jahr 2000 auf die Zeit davor übertragen. Für Fälle, in denen eine Person zu diesem Zeitpunkt aus dem Datensatz ausgeschieden war, musste eine empirische Lösung angewendet werden. Dazu wurde diejenige Ausprägung der ab 2000 erhobenen WZ93-Einteilung eingesetzt, die von anderen Personen des Betriebs mit derselben, bis dahin erfassten WZ73-Ausprägung am häufigsten gewählt wurde.

Darüber hinaus sind Rechtsform und Stellung des Betriebes innerhalb des Unternehmens, sowie die Betriebsgröße, gemessen in der Anzahl der Beschäftigten, und das Bundesland des Betriebes erklärende Variablen des Austrittsgeschehens.

\section{A 2.2.3 Rechtliche Regelungen}

Neben den in den LIAB-Daten selbst enthaltenen Angaben wurden Variablen für die oben beschriebenen Einschränkungen in den Zugangsregeln zur vorgezogenen Altersrente eingeführt. Sie sollen diese in der zweiten Hälfte der 90er Jahre einsetzenden Änderungen kontrollieren und die erschwerten Zugangsmöglichkeiten in die Rente abbilden, die sich in den oben zitierten Studien als wirkungsvolle Maßnahmen zur Verzögerung der Ruhestandsentscheidung erwiesen haben.

Es wurden daher Dummy-Variablen mit dem Wert „1“ erstellt, wenn die Person zum Zeitpunkt des jeweiligen Spells aufgrund ihres Alters zum Bezug einer vorgezogenen Altersrente schon berechtigt war. Dabei wurde unterschieden, ob der Renteneintritt aufgrund der sich ändernden Altersbeschränkungen abschlagfrei, nur mit Abschlägen oder mit maximalen Abschlägen möglich war. Berücksichtigt wurden alle im Beobachtungszeitraum relevanten vorgezogenen Rentenzugangsmöglichkeiten, die in Kapitel A 1.2.1.2 beschrieben wurden. Dies kann wieder nur eine Annäherung an den tatsächlichen Status sein. So lassen sich Abweichungen für den aktuellen Spell nicht vermeiden, weil der Aufbau in Form eines Jahrespanels zu Ungenauigkeiten führen kann. Außerdem ist in den meisten Fällen aus den Daten nicht ersichtlich, ob die neben dem Alter weiteren notwendigen Bedingungen erfüllt waren. Dazu zählen Mindestwartezeiten, ein möglicher Grad der Behinderung oder Mindestpflichtbeitragszeiten.

\section{A 2.3 Selektionsproblematik}

Auch wenn der verwendete Datensatz dem bisher in Deutschland vorhandenen Datenangebot zur Auswertung der betrieblichen Nachfrageseite des Erwerbsaustritts überlegen scheint, so sind auch einige inhärente Schwierigkeiten zu nennen. Sie beziehen sich vorrangig auf die Auswahl der auswertbaren Fälle. Neben den oben angesprochenen besteht ein Problem bei der Auswertung und Interpretation der Ergebnisse in der Selektivität der Daten. Diese ergibt sich aus der Auswahl einerseits der Personen in den beobachteten Betrieben, andererseits der im Panel befindlichen Betriebe. 


\section{A 2.3.1 Selektion auf Personenebene}

Die der Personenebene zugrunde liegende Quelle, die BLH, weist nur sozialversicherungspflichtige Beschäftigung auf. Andere Formen der Erwerbstätigkeit, wie selbstständige Tätigkeiten, eine Anstellung als Beamtin oder abhängige Beschäftigung im Ausland sind nicht beobachtbar. Allerdings verweisen Boockmann und Steffes (2005, S. 8) darauf, dass die Daten der BLH rd. 80\% der Beschäftigten in Deutschland abdecken.

In den Datensatz gehen aus den Geburtsjahrgängen 1945 und früher naturgemäß nur solche Personen ein, die mindestens einmal nach 1995 einer sozialversicherungspflichtigen Beschäftigung nachgegangen sind. Besonders für die frühen Jahrgänge bedeutet dies eine starke Selektion hin zu Personen, die lange Zeit in Beschäftigung verbleiben. Extremfälle, wie 80Jährige, die noch einer Beschäftigung nachgehen, verdeutlichen den selektiven Charakter der vorliegenden Datenbasis. Diese Gründe, insbesondere der letzte, mahnen zur Vorsicht bei der Interpretation der im folgenden Kapitel dokumentierten deskriptiven Ergebnisse und dem Vergleich mit anderen Studien, die ein ähnliches Auswertungsziel verfolgen. Sie dienen ausschließlich der Beschreibung der vorliegenden Datenbasis. Diesem Einwand lässt sich aber entgegenhalten, dass das Auswertungsziel in der Bestimmung der insbesondere betrieblichen Weiterbeschäftigungsgründe liegt. Eine Untersuchung älterer Arbeitnehmer stellt damit die Gültigkeit der Befunde nicht in Frage.

Um die zuletzt beschriebene Positivauswahl dennoch zu mildern, werden im folgenden, deskriptiven Kapitel auch Werte ausgewiesen, die sich auf die Altersgruppe der 1996 50- bis 55Jährigen beschränken. Für die Ergebnisse der Verweildaueranalyse muss daher im Auge behalten werden, dass sich daraus nur Aussagen ableiten, die sich auf die Grundgesamtheit derer beziehen, die im Alter von 50 Jahren und darüber noch sozialversicherungspflichtig beschäftigt sind.

\section{A 2.3.2 Selektion auf Betriebsebene}

Ein weiteres Problem stellt auch die Selektion auf Betriebsebene dar. Diese ergibt sich aus der Auswahl der Betriebe wie sie im IAB-Betriebspanel vorgenommen werden und aus der Tatsache, dass nur Personen mit einem Erwerbstätigkeitsspell in einem zuzuordnenden Betrieb in das LIAB-Jahrespanel eingehen.

Das IAB-Betriebspanel ist eine geschichtete Stichprobe von Betrieben in Deutschland mit sozialversicherungspflichtigen Beschäftigten. Die Schichtung basiert auf Größenklassen und Branchen dieser Betriebe. ${ }^{51}$ Dabei wird ein Oversampling von Groß- und ein Undersampling von Kleinbetrieben vorgenommen. Diese Gewichtung überträgt sich durch die Verknüpfung

\footnotetext{
${ }^{51}$ Vgl. Kölling (2000).
} 
mit dem LIAB-Jahrespanel, da Personen nur dann ausgewertet werden können, wenn Betriebsinformationen für sie vorliegen.

Dass Personen nur dann im Datensatz auftauchen, wenn einigermaßen regelmäßig (vgl. vorangegangenes Kapitel zur Datensatzaufbereitung) die Informationen ihres Betriebes erhoben wurden, stellt ein weiteres Selektionsproblem dar. Da der Betriebswechsel von einem Panelbetrieb in einen anderen nur selten vorkommt, stammt ein nicht-repräsentativ hoher Anteil der Beobachtungen von Personen, die lange in einem Betrieb beschäftigt waren. Insofern liegt eine weitere Positivselektion hin zu Personen mit durchgehenden Erwerbsbiografien vor, für die ein längerer Verbleib im Erwerbsleben vermutet werden kann.

\section{A 2.4 Erwerbsaustritt: Zusammenfassung der Datengrundlage}

Der prozessgenerierte Charakter der Personendaten, der auf die Anforderungen der BA zugeschnitten ist, schimmert an vielen Stellen der Analyse durch und lässt Wünsche von Seiten der Wissenschaft offen. So fehlen in dem verwendeten Datensatz u.a. folgende, wünschenswerte Angaben:

- (Renten-) Versicherungsrelevante Informationen: Zugangsdaten in die Rente, die Art der vorzeitigen Altersrente, Rentenanwartschaften und die von Ehepartnern, Wartezeiten konnten in den Daten so nicht ermittelt werden und mussten - soweit möglich - approximiert werden.

- $\quad$ Gesundheit: Dieses wichtige Eintrittskriterium konnte in den Daten nur als Proxy kontrolliert werden, indem Freistellungszeiten als Indikator für eine gesundheitliche Beeinträchtigung der Erwerbsfähigkeit interpretiert wurden.

- $\quad$ Familiärer Kontext: Auch hier fehlen in den zur Verfügung stehenden Variablen die Angaben sowohl zum (Ehe-) Partner und dessen evtl. Erwerbsstatus, als auch zu Kindern. Aus diesen Gründen bietet sich für weiterführende Arbeiten die Verknüpfung mit den Daten der Rentenversicherer an. Trotzdem bleibt festzuhalten, dass der hier aufbereitete LIABDatensatz aus mehreren Gründen einzigartig ist. Er lässt dank seiner Tiefe an Betriebsinformationen, der Tagesgenauigkeit der prozessgenerierten Personeninformationen und einem beachtlichen Beobachtungsumfang neue Auswertungsmöglichkeiten zu und bietet eine bisher einzigartige Basis zur empirischen Untersuchung von Erwerbstätigkeit im Alter und ihren betrieblichen Einflüssen.

\section{A 3 Deskriptive Ergebnisse}

Im Folgenden sollen die Daten zunächst deskriptiv anhand ausgewählter Variabler analysiert werden. Dabei soll der individuelle Austritt aus dem Erwerbsleben zunächst getrennt nach personen-, dann nach betriebsspezifischen Merkmalen univariat untersucht werden. Um einen besseren Einblick in die Auswirkungen der altersselektiven Stichprobenziehung zu gewinnen, zeigen die folgende Tab. 6 neben dem Anteil an Zensurereignissen - also solchen, bei denen 
kein Übergang in den Ruhestand festgestellt wurde - für das gesamte Sample auch Angaben für einzelne Altersgruppen.

Um in einer verfeinerten deskriptiven Auswertung den möglichen Einfluss individueller wie betrieblicher Variablen auf den Austrittszeitpunkt aus dem Erwerbsleben ausleuchten, wurden zudem Survivor-Funktionen nach dem Kaplan-Meier-Verfahren ${ }^{52}$ geschätzt und über die Zeit abgetragen. Darüber hinaus wurde die Gleichheit dieser Übergangsprozesse für verschiedene Ausprägungen der Variablen jeweils mit Hilfe eines Log-rank-Tests und eines WilcoxonBreslow-Tests überprüft.

Das Kaplan-Meier-Verfahren ist eine nicht-parametrische Schätzmethode zur Bestimmung der Wahrscheinlichkeit, dass ein Zustand über einen Zeitpunkt t hinaus andauert; in dieser Untersuchung, dass bis dahin kein Austrittsereignis zu beobachten ist. Die Schätzung der Survivor-Funktion $\mathrm{S}(\mathrm{t})$ berechnet sich als Produkt der bis zum Zeitpunkt $\mathrm{t}$ geschätzten bedingten Verbleibewahrscheinlichkeiten zu jedem Zeitpunkt eines Übergangs oder einer Zensurbeobachtung. Die Schätzfunktion lautet:

$$
\widehat{S}(t)=\prod_{j \mid t_{j} \leq t}\left(\frac{n_{j}-d_{j}}{n_{j}}\right),
$$

wobei $n_{j}$ den Umfang der Risikogruppe zum Zeitpunkt $t_{j}$ angibt und $d_{j}$ die Anzahl der Austritte. Die Risikogruppe setzt sich zusammen aus all den Personen, für die zum jeweiligen Zeitpunkt ein Austritt beobachtet werden kann. Dies schließt Personen aus, die schon zuvor aus der Beobachtung - durch ein Austrittsereignis oder ein Ausscheiden aus der Stichprobe (Rechtszensierung) - ausgeschieden sind, oder solche, die erst nach dem Zeitpunkt in die Stichprobe eingehen (Linkszensierung).

Um Unterschiede zwischen gruppenspezifischen Survivor-Funktionen zu untersuchen, können eine Reihe von Tests durchgeführt werden, die alle nach demselben Prinzip funktionieren: Verglichen wird der gesamte Verlauf dieser Survivor-Funktionen über die Auswertungszeit hinweg. Unter der Nullhypothese

$$
\mathrm{H}_{0}: \mathrm{S}_{1}(\mathrm{t})=\mathrm{S}_{2}(\mathrm{t})=\ldots=\mathrm{S}_{\mathrm{r}}(\mathrm{t})
$$

die die Gleichheit der allgemein r Funktionen beinhaltet, ist die Teststatistik $\chi^{2}$ verteilt mit r -1 Freiheitsgraden. Den Ausgangspunkt stellen Kontingenztabellen für jeden Zeitpunkt dar, in denen für jede Gruppe die Anzahl der Austritte und der Personen in der Risikogruppe abgetragen wird.

\footnotetext{
${ }^{52}$ Eine ausführliche Beschreibung der nicht-parametrischen Schätzung der Survivor-Funktion und von Tests der Gleichheit zweier solcher Funktionen findet sich z.B. bei Blossfeld et al. (1986) S. 42 ff. und Cleves et al. (2004) S.91 ff.
} 
Die hier verwendeten Tests auf Gleichheit der Survivor-Funktionen beruhen auf einem Rangsummentestprinzip. Ihnen ist gemein, dass für jeden kritischen Zeitpunkt $t_{j}$ eine Kontingenztabelle erstellt wird, in der für die $r$ verschiedenen Ausprägungen der bedingenden Variablen die Zahl der Austritte $\left(\mathrm{d}_{1 \mathrm{j}}, \mathrm{d}_{2 \mathrm{j}}, \ldots \mathrm{d}_{\mathrm{rj}}\right)$, die Zahl der verbleibenden Individuen $\left(\mathrm{n}_{1 \mathrm{j}}-\mathrm{d}_{1 \mathrm{j}}, \mathrm{n}_{2 \mathrm{j}}-\mathrm{d}_{2 \mathrm{j}}\right.$, $\left.\ldots \mathrm{n}_{\mathrm{rj}}-\mathrm{d}_{\mathrm{rj}}\right)$ und der Personen in der Risikogruppe $\left(\mathrm{n}_{1 \mathrm{j}}, \mathrm{n}_{2 \mathrm{j}}, \ldots \mathrm{n}_{\mathrm{rj}}\right)$ dargestellt wird. Unter der Nullhypothese der Gleichheit der Funktionsverläufe ergibt sich eine gruppenspezifische erwartete Anzahl von Übergängen zum Zeitpunkt $t_{j}$ als $E_{i j}=n_{i j} d_{j} / n_{j}$. Die $\chi^{2}$-verteilte Teststatistik $\mathrm{u}^{\prime} \mathrm{V}^{-1} \mathrm{u}$ ergibt sich dann aus dem Zeilenvektor

$$
\mathrm{u}^{\prime}=\sum_{\mathrm{j}=1}^{\mathrm{k}} W\left(t_{j}\right)\left(d_{1 j}-E_{1 j}, \ldots, d_{r j}-E_{r j}\right)
$$

und der r×r- Varianz-Kovarianz-Matrix mit ihren Elementen $\mathrm{V}_{\mathrm{il}}$

$$
V_{i l}=\sum_{j=1}^{k} \frac{W^{2}\left(t_{j}\right) n_{i j} d_{j}\left(n_{j}-d_{j}\right)}{n_{j}\left(n_{j}-1\right)}\left(\delta_{i l}-\frac{n_{i j}}{n_{j}}\right),
$$

wobei $\mathrm{i}=1, \ldots, r, 1=1, \ldots, \mathrm{r}$ und $\delta_{\mathrm{ij}}=1$, falls $\mathrm{i}=1$, sonst 0 .

Wichtig bleibt zu erwähnen, dass sich die Tests auf den gesamten Verlauf der SurvivorFunktionen beziehen und nicht auf einzelne Zeitpunkte des Übergangsprozesses beschränkt sind. Die aus diesen allgemeinen Formeln ableitbaren Tests unterscheiden sich ausschließlich in der Gewichtungsfunktion W charakterisiert den einzelnen Test. Der Log-rank-Test gewichtet dabei alle Übergangszeitpunkte identisch mit $\mathrm{W}\left(\mathrm{t}_{\mathrm{j}}\right)=1$, solange die Risikomenge positiv ist. Der ebenfalls mit angegebene Wilcoxon-Test legt dagegen stärkeres Gewicht auf frühe Übergangszeitpunkte, indem die Gewichtung als $\mathrm{W}\left(\mathrm{t}_{\mathrm{j}}\right)=\mathrm{n}_{\mathrm{j}}$ gewählt wird.

\section{A 3.1 Austritt nach persönlichen Merkmalen}

Im ersten Schritt soll der Verbleib in Beschäftigung dargestellt werden. Für den Gesamtdatensatz von knapp 800000 Personen sind in 37,6 \% der Fälle keine Austritte nach dem oben beschriebenen Kriterium zu verzeichnen gewesen. Die Unterteilung nach Altersgruppe illustriert die Auswirkungen der Auswahl von Personen, die trotz fortgeschrittenen Alters noch in Beschäftigung sind. So sinkt der Anteil der bis Ende 2003 in Beschäftigung Befindlichen unter den 56-60-Jährigen im Vergleich mit den 50-55-Jährigen stark. Für die 1996 Über-60Jährigen steigt diese Quote auf bis 43,1\% bei der Gruppe der Über-65-Jährigen, ein Befund der sich auch nach Kontrolle über die Geschlechts-, Nationalitäten- und Regionalvariable beibehalten lässt. Ausschlaggebend hierfür dürfte die genannte Selektionsproblematik sein: die Auswahl dieser Personengruppe bringt mit sich, dass es sich um Personen handelt, die eine geringe Neigung zum Austritt haben. Eine Erklärung bietet auch ein Blick auf die Zensuranteile nach Kontrolle für die Ausbildung oder den Berufsstatus. Dabei fällt auf, dass sich der 
Anteil der Zensurereignisse für die Über-65-Jährigen ${ }^{53}$ in einigen Fällen über dem Gesamtanteil bewegt. Diese ältesten Erwerbstätigen verfügen danach oft nur über eine geringe formale Schulbildung und sind meist in Teilzeit unter 15 Stunden pro Woche beschäftigt.

Dies lässt sich als Indiz dafür interpretieren, dass die selektive Auswahl auch auf beobachtbaren Variablen beruht. Auch muss darauf verwiesen werden, dass es sich bei den Altersgruppen ab 61 Jahren um eine im Gesamtdatensatz recht unbedeutende Gruppe handelt. Sie machen nur rd. fünf Prozent aller beobachteten Personen aus.

Geschlecht: Eine Unterscheidung der Zensuranteile nach dem Geschlecht bringt zum Vorschein, dass Männer deutlich seltener im Beobachtungszeitraum aus dem Erwerbsleben ausscheiden als Frauen. Das Verhältnis kehrt sich jedoch um, wenn man die Kohorten der im Jahr 1996 56-Jährigen und Älteren betrachtet. Für die ausscheidenden Männer lässt sich ein entsprechend höheres durchschnittliches Austrittsalter als bei den Frauen beobachten (vgl. Tab. 20 im Anhang A). Der Vergleich der beiden Überlebensfunktionen in Tab. und Abb. 28 ergibt ebenfalls, dass sich Frauen signifikant früher aus dem Erwerbsleben zurückziehen.

Region: Der Vergleich zwischen Ost- und Westdeutschland bringt eine große Differenz zwischen den Landesteilen ans Licht. Danach treten im Beobachtungszeitraum knapp 60 Prozent der Westdeutschen aus dem Erwerbsleben aus, während der Anteil unter den Ostdeutschen über 68 Prozent beträgt. Dies drückt sich auch darin aus, dass das durchschnittliche Alter des beobachteten Austritts in den neuen Bundesländern hinter dem Wert für Westdeutschland zurückbleibt. Die Ergebnisse lassen sich auch anhand des optischen und des analytischen Vergleichs der Survivor-Funktionen (Abb. 29 im Anhang A) bestätigen, die einen hochsignifikanten Unterschied zwischen den beiden Bevölkerungsgruppen bescheinigen.

Bei diesen ersten beiden Variablen handelt es sich um die Merkmale, hinsichtlich derer die multivariate Auswertung aufgeteilt wird. Angesichts der jeweils deutlichen Unterschiede im Austrittsverhalten lässt sich eine separate Schätzung auch empirisch rechtfertigen.

Nationalität: Die Unterscheidung nach Staatsangehörigkeit der potentiellen Rentner ergibt, dass Deutsche in jeder Altersgruppe geringere Austrittsquoten aufweisen als Personen ohne deutschen Pass. Für die beobachteten deutschen Austrittsfälle lässt sich ein späteres Austrittsalter feststellen.

Ausbildung: Uneinheitlicher wirkt sich die formale Ausbildung aus. Die höchsten Übergangsraten sind für Personen mit niedriger formaler Ausbildung zu verzeichnen. Dagegen treten Universitätsabsolventen am seltensten aus dem Erwerbsleben aus. Allerdings steigen die Austrittsquoten von Akademikern mit zunehmendem Alter, während diejenigen von geringer

\footnotetext{
${ }^{53}$ Man beachte, dass im Jahr 1996 66-Jährige im letzten möglichen Austrittsjahr immerhin schon 73 Jahre alt waren.
} 
Tab. 6: Anteil an nicht beobachteten Austritten nach Altersgruppe im Jahr 1996 und Personenvariablen [in \%]

\begin{tabular}{|c|c|c|c|c|c|c|c|c|c|c|}
\hline \multirow{2}{*}{$\begin{array}{l}\text { Altersgruppe } \\
\text { Variable }\end{array}$} & \multicolumn{2}{|c|}{ alle } & \multicolumn{2}{|c|}{$50-55$} & \multicolumn{2}{|c|}{$56-60$} & \multicolumn{2}{|c|}{$61-65$} & \multicolumn{2}{|c|}{ Älter als 65} \\
\hline & $\%$ & $\mathbf{n}$ & $\%$ & $\mathbf{n}$ & $\%$ & $\mathbf{n}$ & $\%$ & $\mathbf{N}$ & $\%$ & $\mathbf{n}$ \\
\hline Alle & 37,6 & 781131 & 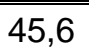 & 545299 & 16,2 & 195548 & 28,6 & 28009 & 43,1 & 12275 \\
\hline \multicolumn{11}{|l|}{ Geschlecht } \\
\hline Mann & 38,8 & 487782 & 48,0 & 337120 & 15,8 & 124567 & 23,9 & 19347 & 38,8 & 6748 \\
\hline Frau & 35,6 & 293349 & 41,5 & 208179 & 16,9 & 70981 & 39,1 & 8662 & 43,8 & 5527 \\
\hline \multicolumn{11}{|l|}{ Nationalität } \\
\hline Deutsch & 37,6 & 739502 & 45,6 & 514470 & 16,3 & 186648 & 29,2 & 26387 & 43,3 & 11997 \\
\hline Nicht deutsch & 37,0 & 41629 & 44,1 & 30829 & 15,6 & 8900 & 18,4 & 1622 & 38,1 & 278 \\
\hline \multicolumn{11}{|l|}{ Region } \\
\hline Ost & 31,7 & 275310 & 38,6 & 199115 & 11,7 & 66007 & 21,8 & 7564 & 34,9 & 2624 \\
\hline West & 40,8 & 505820 & 49,5 & 346184 & 18,6 & 129540 & 31,1 & 20445 & 45,4 & 9651 \\
\hline \multicolumn{11}{|l|}{$\begin{array}{l}\text { Ausbildung } \\
\text { bis mittlere Reife ohne }\end{array}$} \\
\hline $\begin{array}{l}\text { Berufsausbildung } \\
\text { bis mittlere Reife mit Be- }\end{array}$ & 32,2 & 124502 & 39,4 & 84282 & 13,6 & 33062 & 27,9 & 4714 & 41,5 & 2444 \\
\hline $\begin{array}{l}\text { rufsausbildung } \\
\text { (Fach-) Hochschulreife }\end{array}$ & 34,8 & 445950 & 43,1 & 327126 & 10,0 & 104427 & 22,0 & 10718 & 42,8 & 3679 \\
\hline $\begin{array}{l}\text { ohne Berufsausbildung } \\
\text { (Fach-) Hochschulreife }\end{array}$ & 43,0 & 3219 & 56,2 & 2176 & 14,8 & 843 & 13,2 & 167 & 39,4 & 33 \\
\hline mit Berufsausbildung & 40,2 & 15524 & 51,6 & 11045 & 11,3 & 3877 & 13,5 & 465 & 25,5 & 137 \\
\hline Fachhochschulabschluss & 40,8 & 36809 & 53,1 & 26548 & 9,2 & 9153 & 6,8 & 1022 & 27,9 & 86 \\
\hline Hochschulabschluss & 47,6 & 49442 & 65,0 & 32348 & 16,0 & 14094 & 7,2 & 2749 & 22,3 & 251 \\
\hline $\begin{array}{l}\text { fehlt/keine Angabe } \\
\text { Berufliche Stellung }\end{array}$ & 49,5 & 104989 & 52,5 & 61481 & 44,2 & 29755 & 49,2 & 8112 & 45,7 & 5641 \\
\hline Auszubildende & 24,0 & 196 & 32,5 & 80 & 16,8 & 95 & 28,6 & 14 & 14,3 & 7 \\
\hline Einfache Arbeiter & 27,3 & 112060 & 34,5 & 79899 & 8,1 & 28679 & 16,9 & 2859 & 31,1 & 623 \\
\hline Facharbeiter & 27,2 & 100012 & 35,4 & 73822 & 3,8 & 24241 & 7,0 & 1781 & 31,0 & 168 \\
\hline Meister u.a. & 27,4 & 12305 & 38,6 & 8294 & 3,9 & 3557 & 2,4 & 422 & 37,5 & 32 \\
\hline Angestellte & 32,6 & 245662 & 44,7 & 167297 & 6,7 & 69785 & 4,1 & 8011 & 32,3 & 569 \\
\hline Teilzeit < 15h & 59,4 & 134466 & 67,0 & 71247 & 52,3 & 40848 & 50,3 & 12979 & 45,8 & 9756 \\
\hline Teilzeit > 15h & 41,1 & 174908 & 47,8 & 143953 & 8,4 & 28183 & 28,4 & 1803 & 34,4 & 969 \\
\hline
\end{tabular}

Quelle: LIAB, eigene Berechnungen

Qualifizierten z.T. deutlich weniger stark ansteigen. Festzuhalten bleibt auch die Kategorie derjenigen, für die Angaben zum Bildungsabschluss fehlen. Diese zahlenmäßig recht bedeutende Gruppe verzeichnet durchgängig ein hohe Zensurquote und ein überdurchschnittliches Austrittsalter auf.

Es lässt sich zusammenfassen, dass Personen mit hohem Bildungsabschluss ein vergleichsweise einheitliches Austrittsalter nahe dem gesetzlichen Renteneintrittsalter wählen, während Personen mit niedrigeren Abschlüssen in höherem Maße früh austreten oder aber das Ende der Erwerbstätigkeit verschieben. Sowohl der Log-rank-Test und als auch der Wilcoxon-Test in Tab. bestätigen die zunächst rein deskriptive Analyse, nach der sich die Austrittsmuster von Personen unterschiedlicher formaler Bildung unterscheiden. 
Tab. 7: $\quad$ Tests auf Gleichheit der Survivor-Funktionen nach persönlichen Merkmalen

\begin{tabular}{|c|c|c|c|c|}
\hline & $\begin{array}{c}\text { Events } \\
\text { Observed } \\
\end{array}$ & $\begin{array}{c}\text { Events } \\
\text { Expected } \\
\end{array}$ & $\begin{array}{l}\text { Sum of } \\
\text { Ranks }\end{array}$ & \\
\hline \multicolumn{5}{|l|}{ Geschlecht } \\
\hline Frau & 129484 & 121097,11 & & \multirow{3}{*}{$\begin{array}{l}\text { Log-rank Test: } \\
X^{2}(1)=923,84 \\
\text { Pr }>X^{2}=0,0000 \\
\text { Wilcoxon Test: } \\
X^{2}(1)=928,81 \\
\operatorname{Pr}>X^{2}=0,0000\end{array}$} \\
\hline Mann & 202564 & 210950,89 & $-4,267 e+09$ & \\
\hline Gesamt & 332048 & 332048,00 & & \\
\hline \multicolumn{5}{|l|}{ Region } \\
\hline Ostdeutschland & 127405 & 103114,00 & $1,301 e+10$ & \multirow{3}{*}{$\begin{array}{l}\text { Log-rank-Test: } \\
X^{2}(1)=8408,29 \\
\text { Pr }>X^{2}=0,0000 \\
\text { Wilcoxon-Test: } \\
X^{2}(1)=9142,14 \\
\operatorname{Pr}>X^{2}=\mathbf{0 , 0 0 0 0}\end{array}$} \\
\hline Westdeutschland & 204643 & 228934,00 & $-1,301 e+10$ & \\
\hline Gesamt & 332048 & 332048,00 & & \\
\hline \multicolumn{5}{|l|}{ Ausbildung } \\
\hline Bis mittlere Reife ohne BA & 60137 & 54288,00 & $2,775 e+09$ & \multirow{7}{*}{$\begin{array}{l}\text { Log-rank-Test: } \\
X^{2}(5)=2926,97 \\
\text { Pr }>X^{2}=0,0000 \\
\text { Wilcoxon-Test: } \\
X^{2}(5)=2511,38 \\
\operatorname{Pr}>X^{2}=0,0000\end{array}$} \\
\hline Bis mittlere Reife mit BA & 203555 & 199529,44 & $1,181 e+09$ & \\
\hline Hochschulreife ohne BA & 1180 & 1489,29 & $-1,346 e+09$ & \\
\hline Hochschulreife mit BA & 6331 & 7188,66 & $-3,741 e+08$ & \\
\hline Fachhochschulabschluss & 15418 & 17662,76 & $-7,789 e+08$ & \\
\hline Hochschulabschluss & 17670 & 24132,85 & $-2,669 e+09$ & \\
\hline Gesamt & 304291 & 304291,00 & & \\
\hline \multicolumn{5}{|l|}{ Berufliche Stellung } \\
\hline Auszubildende & 99 & 67,91 & 25747769 & \multirow{8}{*}{$\begin{array}{l}\text { Log-rank-Test: } \\
X^{2}(6)=9507,82 \\
\text { Pr }>X^{2}=0,0000 \\
\text { Wilcoxon-Test: } \\
X^{2}(6)=13064,23 \\
\operatorname{Pr}>X^{2}=0,0000\end{array}$} \\
\hline Einfache Arbeiter & 59294 & 49934,38 & $5,102 e+09$ & \\
\hline Facharbeiter & 52818 & 46444,08 & $3,900 e+09$ & \\
\hline Meister u.a. & 6440 & 6056,08 & $2,397 e+08$ & \\
\hline Angestellte & 118958 & 123490,05 & $-1,006 e+09$ & \\
\hline Teilzeit $<15 \mathrm{~h}$ & 23891 & 38588,15 & $-8,736 e+09$ & \\
\hline Teilzeit $>15 h$ & 69699 & 65618,43 & $4,742 \mathrm{e}+08$ & \\
\hline Gesamt & 331199 & 331199,00 & & \\
\hline \multicolumn{5}{|l|}{ Eintritt in Betrieb } \\
\hline Eintritt $\leq 1975$ & 87383 & 71197,04 & $8,364 \mathrm{e}+09$ & \multirow{5}{*}{$\begin{array}{l}\text { Log-rank-Test: } \\
X^{2}(3)=5158,64 \\
\text { Pr }>X^{2}=\mathbf{0 , 0 0 0 0} \\
\text { Wilcoxon-Test: } \\
X^{2}(3)=5306,38 \\
\operatorname{Pr}>X^{2}=\mathbf{0 , 0 0 0 0}\end{array}$} \\
\hline $1975<$ Eintritt $\leq 1980$ & 27310 & 27132,26 & -22878761 & \\
\hline $1980<$ Eintritt $\leq 1990$ & 36846 & 42974,17 & $-3,334 e+09$ & \\
\hline Eintritt > 1990 & 180509 & 190744,53 & $-5,006 e+09$ & \\
\hline Gesamt & 332048 & 332048,00 & & \\
\hline \multicolumn{5}{|l|}{ Eintritt in Erwerbsleben } \\
\hline Eintritt $\leq 1975$ & 158195 & 162024,37 & $-2,247 e+09$ & \multirow{4}{*}{$\begin{array}{l}\text { Log-rank-Test: } \\
X^{2}(3)=4777,84 \\
\text { Pr }>X^{2}=\mathbf{0 , 0 0 0 0} \\
\text { Wilcoxon-Test: } \\
X^{2}(3)=5326,16 \\
\operatorname{Pr}>X^{2}=\mathbf{0 , 0 0 0 0}\end{array}$} \\
\hline $1975<$ Eintritt $\leq 1980$ & 23265 & 29229,81 & $-3,119 e+09$ & \\
\hline $1980<$ Eintritt $\leq 1990$ & 13036 & 18366,29 & $-2,786 e+09$ & \\
\hline $\begin{array}{l}\text { Eintritt }>1990 \\
\text { Gesamt }\end{array}$ & $\begin{array}{l}137552 \\
332048\end{array}$ & $\begin{array}{l}122427,53 \\
332048,00\end{array}$ & $8,151 e+09$ & \\
\hline \multicolumn{5}{|l|}{ Tagesentgelt } \\
\hline Entgelt $\leq 100 €$ & 159376 & 178213,18 & $-1,169 e+10$ & Log-rank-Test: \\
\hline $100 €<$ Entgelt $\leq 200 €$ & 96144 & 97996,20 & $4,253 e+08$ & $X^{2}(3)=15113,00$ \\
\hline $200 €<$ Entgelt $\leq 300 €$ & 24012 & 23571,87 & $6,461 e+08$ & $\operatorname{Pr}>x^{2}=0,0000$ \\
\hline Entgelt $>300 €$ & 52516 & 32266,75 & $1,062 e+10$ & Wilcoxon-Test: \\
\hline Gesamt & 332048 & 332048,00 & & $\begin{array}{l}X^{2}(3)=16363,91 \\
\operatorname{Pr}>X^{2}=\mathbf{0 , 0 0 0 0}\end{array}$ \\
\hline
\end{tabular}

Quelle: LIAB, eigene Berechnungen 
Berufliche Stellung: Auch was die berufliche Stellung angeht, unterscheiden sich die Personen der Stichprobe. Während Arbeiter und Meister geringe Verbleibequoten in der sozialversicherungspflichtigen Erwerbstätigkeit aufweisen, ist der Anteil unter den Angestellten recht hoch. Dies schlägt sich allerdings nicht in einem auffallend hohen durchschnittlichen Austrittsalter der Angestellten nieder. Besonders auffällig ist wieder das Austrittsverhalten von Personen mit Teilzeitarbeit, die hier als Kategorie der beruflichen Stellung auftaucht. Sie zeichnen sich durch sehr niedrige Austrittswahrscheinlichkeiten und ein hohes durchschnittliches Austrittsalter aus. Möglicherweise ist dies auf sozialversicherungspflichtige Tätigkeiten von vorzeitigen Altersrentnern zurückzuführen, die - wie oben beschrieben - in ihrem Hinzuverdienst beschränkt sind und daher eine Teilzeitstelle annehmen. Wieder bestätigt die induktive Auswertung diese Ergebnisse: die Testwerte in Tab. zeigen, dass Arbeiter früher als Angestellte den Arbeitsmarkt verlassen.

Eintritt in den Betrieb: Eine weitere wichtige Humankapitalvariable stellt das Eintrittsalter in den Betrieb dar. Die Ergebnisse der Kaplan-Meier-Schätzung sind in Abb. 34 im Anhang A veranschaulicht. Danach ergibt sich, dass tendenziell Personen mit einem frühen Eintritt in den Betrieb einer größeren Gefahr des Austritts ausgesetzt sind. Allerdings kann man davon ausgehen, dass die Variable stark mit dem Alter korreliert und erst in einer multivariaten Auswertung verlässliche Ergebnisse hinsichtlich des Einflusses der Betriebszugehörigkeitsdauer zu erwarten sind.

Auch sei daran erinnert, dass die Werte für Ostdeutsche erst mit dem Jahr 1990 beginnen. Dies mag die durchweg höhere Verbleibewahrscheinlichkeit der zwischen 1980 und 1990 in ihren Betrieb Eingetretenen gegenüber den Personen mit kürzerer Zugehörigkeit erklären.

Eintritt in das Erwerbsleben: Wie aus Tab. und Abb. 34 erkennbar ist, sind Personen mit frühem Eintrittsdatum einem höheren Risiko des Austritts ausgesetzt. Dies lässt sich wie im Falle der Betriebszugehörigkeitsdauer durch die Korrelation mit der Altersvariablen erklären. Auch in dieser Unterteilung der Stichprobe ragen die Personen mit einem Eintritt in das Erwerbsleben nach 1990 heraus. Wieder ist aufgrund der zensierten Beobachtungswerte für die ostdeutschen Versicherten davon auszugehen, dass die geringen Werte der geschätzten Survivor-Funktion dieser letzten Kategorie von diesen Unzulänglichkeiten der Daten mit beeinflusst sind. Die anschließende, nach Landesteilen getrennte Hazardraten-Schätzung wird die von der Erwerbsdauer ausgehenden Effekte zuverlässiger ermitteln können.

Tagesentgelt: Der Einfluss des Tagesentgeltes ist theoretisch zunächst unbestimmt. Einerseits erhöht er die Opportunitätskosten des Austritts für den Versicherten und wirkt daher verlängernd auf den aktiven Erwerbsstatus, andererseits steigt c.p. das Interesse des Betriebes, diesen Beschäftigten in den (vorzeitigen) Ruhestand zu entlassen und dadurch Kosten einzusparen. Abb. 34 gibt die Ergebnisse der Survivor-Funktionen getrennt nach Klassen des imputierten Tagesentgeltes wider. Danach sind die Überlebenswahrscheinlichkeiten von Geringverdienern zu jedem Zeitpunkt am niedrigsten. $\mathrm{Zu}$ beachten ist, dass es sich hierbei um 
eine zeitveränderliche Variable handelt, die Kurven demnach den Übergangsprozess einer Personengruppe beschreibt, die durchgängig in einer der Einkommensklassen bleibt.

Aktuelle Freistellung: Um für den Gesundheitszustand zu kontrollieren, wurde eine DummyVariable gebildet, die angibt, ob eine Person in den beiden Jahren vor dem aktuellen Spellende einen Freistellungszeitraum aufwies. Hier zeigen sich deutlich die in anderen empirischen Untersuchungen nachgewiesenen Effekte. Nach Abb. 35 bleiben Personen mit einer solchen Unterbrechung mit wesentlich geringerer Wahrscheinlichkeit dem Arbeitsmarkt erhalten als ihre Kollegen, die keine aktuellen Freistellungszeiten aufzuweisen hatten. Die Tests in Tab. auf Gleichheit der Survivor-Funktionen kommen zum selben Ergebnis. Wieder ist anzumerken, dass es sich nicht um eine zeitkonstante Variable handelt und damit nur wenige Ausnahmefälle beschreibt.

\section{A 3.2 Austritt nach betrieblichen Merkmalen}

Die Zensurquote und das durchschnittliche Austrittsalter ähnlich den Personenvariablen aufzubereiten, erscheint für die Betriebsvariablen nicht sinnvoll, da es sich meist um zeitlich veränderliche Variable handelt. Damit ist der Bezug auf einen einzelnen, beliebig gewählten Zeitpunkt nicht aussagekräftig. Stattdessen werden hier Kaplan-Meier-Schätzungen für die einzelnen Ausprägungen der betrieblichen Variablen dargestellt und die Differenzen der Survivor-Funktionen getestet. Dabei handelt es sich allerdings nicht um den Vergleich realer Beobachtungen, sondern um den Vergleich hypothetischer Gruppen von Personen, die durchgängig dieselben Werte der Betriebsvariablen aufweisen.

\section{A 3.2.1 Institutionen}

Zunächst soll der Block der Variablen beschrieben werden, die auf institutionelle Hindernisse bei der betrieblichen Entscheidung gegen eine Weiterbeschäftigung von Älteren hindeuten. Stattdessen ist ein verlängernder Einfluss auf die Erwerbstätigkeit zu vermuten.

Tarifbindung: In der univariaten Analyse bestätigen sich die Erwartungen nicht, dass die Tarifbindung eines Betriebes die Verbleibewahrscheinlichkeit Älterer im Betrieb erhöht. Wie aus Abb. 7 ersichtlich, liegt die Survivor-Funktion von Personen, die in einem Betrieb ohne jede Tarifbindung arbeiten, deutlich über der von „geschützten“ Arbeitnehmern in Betrieben mit Branchen- oder Haustarifvertrag. Auch die Tests aus Tab. unterstreichen mit signifikanten Ergebnissen den Unterschied der Schätzfunktionen. Allerdings kann eingewandt werden, dass dieses Ergebnis nicht ursächlich dem Kriterium „Tarifvertrag“ angelastet werden kann, sondern auf Korrelationen mit weiteren Einflussgrößen zurückzuführen ist. Die multivariate Hazardraten-Schätzung wird diesen Aspekt erhellen und zeigen, ob der Zusammenhang auch nach Kontrolle für weitere Regressoren dieselbe Richtung aufweist. 
Tab. 8: $\quad$ Tests auf Gleichheit der Survivor-Funktionen nach betrieblichen Merkmalen

\begin{tabular}{|c|c|c|c|c|}
\hline & $\begin{array}{l}\text { Events } \\
\text { Observed }\end{array}$ & $\begin{array}{l}\text { Events } \\
\text { expected }\end{array}$ & $\begin{array}{l}\text { Sum of } \\
\text { Ranks }\end{array}$ & \\
\hline \multicolumn{5}{|l|}{ Tarifbindung } \\
\hline Branchentarifvertrag & 248742 & 245461,35 & $3,082 e+09$ & \multirow{4}{*}{$\begin{array}{l}\text { Log-rank Test: } \\
X^{2}(2)=1433,82 \\
\operatorname{Pr}>X^{2}=\mathbf{0 , 0 0 0 0} \\
\text { Wilcox on Test: } \\
X^{2}(2)=2406,45 \\
\operatorname{Pr}>X^{2}=\mathbf{0 , 0 0 0 0}\end{array}$} \\
\hline Haus- bzw. Firmentarifvertrag & 46305 & 42976,63 & $1,255 e+09$ & \\
\hline Kein Tarifvertrag & 32542 & 39151,02 & $-4,337 e+09$ & \\
\hline Gesamt & 327589 & 327589 & & \\
\hline \multicolumn{5}{|l|}{ Mitbestimmung } \\
\hline Kein Betriebsrat & 39852 & 41209,25 & $-1,589 e+09$ & \multirow{3}{*}{$\begin{array}{l}\text { Log-rank-Test: } \\
X^{2}(1)=53,15 \\
\text { Pr }>X^{2}=0,0000 \\
\text { Wilcoxon-Test: } \\
X^{2}(1)=366,95 \\
\operatorname{Pr}>X^{2}=0,0000\end{array}$} \\
\hline Betriebsrat & 236277 & 234919,75 & $1,589 e+09$ & \\
\hline Gesamt & 276129 & 276129,00 & & \\
\hline \multicolumn{5}{|l|}{ Kündigungsschutz } \\
\hline $\begin{array}{l}\text { Keine Geltung des Kündi- } \\
\text { gungsschutzgesetzes }\end{array}$ & 3159 & 4513,74 & $-6,991 e+08$ & \multirow{4}{*}{$\begin{array}{l}\text { Log-rank-Test: } \\
X^{2}(1)=416,77 \\
\operatorname{Pr}>X^{2}=0,0000 \\
\text { Wilcoxon-Test: } \\
X^{2}(1)=469,04 \\
P r>X^{2}=0,0000\end{array}$} \\
\hline $\begin{array}{l}\text { Geltung des Kündigungs- } \\
\text { schutzgesetzes }\end{array}$ & 325992 & 324637,26 & $6,991 \mathrm{e}+08$ & \\
\hline Gesamt & 329151 & 329151,00 & & \\
\hline \multicolumn{4}{|l|}{ Befristete Beschäftigung } & \\
\hline Keine befristet. Beschäftigten & 55540 & 59630,10 & $-2,041 e+09$ & \multirow{6}{*}{$\begin{array}{l}\text { Log-rank-Test: } \\
X^{2}(4)=4294,04 \\
\operatorname{Pr}>X^{2}=0,0000 \\
\text { Wilcoxon-Test: } \\
X^{2}(4)=4599,14 \\
\operatorname{Pr}>X^{2}=0,0000\end{array}$} \\
\hline Anteil $<2 \%$ & 88231 & 80878,23 & $4,468 e+09$ & \\
\hline $2 \%<$ Anteil < $5 \%$ & 65475 & 70420,65 & $-2,190 e+09$ & \\
\hline $5 \%<$ Anteil $<10 \%$ & 42060 & 50640,55 & $-4,843 e+09$ & \\
\hline Anteil $>10 \%$ & 80742 & 70478,47 & $-4,606 e+09$ & \\
\hline Gesamt & 332048 & 332048,00 & & \\
\hline \multicolumn{5}{|l|}{ Anteil Bis-30-Jähriger } \\
\hline Anteil $<10 \%$ & 72194 & 67501,63 & $1,601 e+09$ & \multirow{6}{*}{$\begin{array}{l}\text { Log-rank-Test: } \\
X^{2}(4)=1605,96 \\
\text { Pr> } X^{2}=0,0000 \\
\text { Wilcoxon-Test: } \\
X^{2}(4)=1408,66 \\
\operatorname{Pr}>X^{2}=\mathbf{0 , 0 0 0 0}\end{array}$} \\
\hline $10 \%<$ Anteil $<15 \%$ & 69503 & 63225,42 & $3,420 e+09$ & \\
\hline $15 \%<$ Anteil $<20 \%$ & 75936 & 78954,73 & $-1,340 e+09$ & \\
\hline $20 \%<$ Anteil $<25 \%$ & 57030 & 61220,33 & $-1,677 e+09$ & \\
\hline Anteil $>30 \%$ & 57385 & 61145,90 & $-2,004 e+09$ & \\
\hline Gesamt & 332048 & 332048,00 & & \\
\hline \multicolumn{5}{|c|}{ Investitionen in Kommunikationstechnik } \\
\hline keine Investitionen & 74003 & 68873,09 & $2,817 e+09$ & \multirow{3}{*}{$\begin{array}{l}\text { Log-rank-Test: } \\
X^{2}(1)=491,04 \\
\text { Pr }>X^{2}=0,0000 \\
\text { Wilcoxon-Test: } \\
X^{2}(1)=559,12 \\
\operatorname{Pr}>X^{2}=0,0000\end{array}$} \\
\hline Investitionen & 253137 & 258266,91 & $-2,817 e+09$ & \\
\hline Gesamt & 327140 & 327140,00 & & \\
\hline \multicolumn{5}{|c|}{ Betriebsgröße [Anzahl Beschäftigte] } \\
\hline Anzahl $<200$ & 53233 & 68003,35 & $-8,852 e+09$ & \multirow{6}{*}{$\begin{array}{l}\text { Log-rank-Test: } \\
X^{2}(4)=6438,49 \\
\text { Pr }>X^{2}=0,0000 \\
\text { Wilcoxon-Test: } \\
X^{2}(4)=9280,57 \\
P r>X^{2}=0,0000\end{array}$} \\
\hline $200<$ Anzahl $<1000$ & 117295 & 122510,77 & $-3,357 e+09$ & \\
\hline $1000<$ Anzahl $<2000$ & 63858 & 56607,42 & $5,249 e+09$ & \\
\hline $2000<$ Anzahl $<5000$ & 49110 & 43833,97 & $3,857 e+09$ & \\
\hline Anzahl > 5000 & 48552 & 41092,49 & $3,102 e+09$ & \\
\hline Gesamt & 332048 & 332048,00 & & \\
\hline
\end{tabular}

Quelle: LIAB, eigene Berechnungen 
Abb. 7:

Kaplan-Meier-Schätzung - Tarifbindung

Kaplan-Meier: Überleben in sv-pflichtiger Beschäftigung nach Tarifbindung

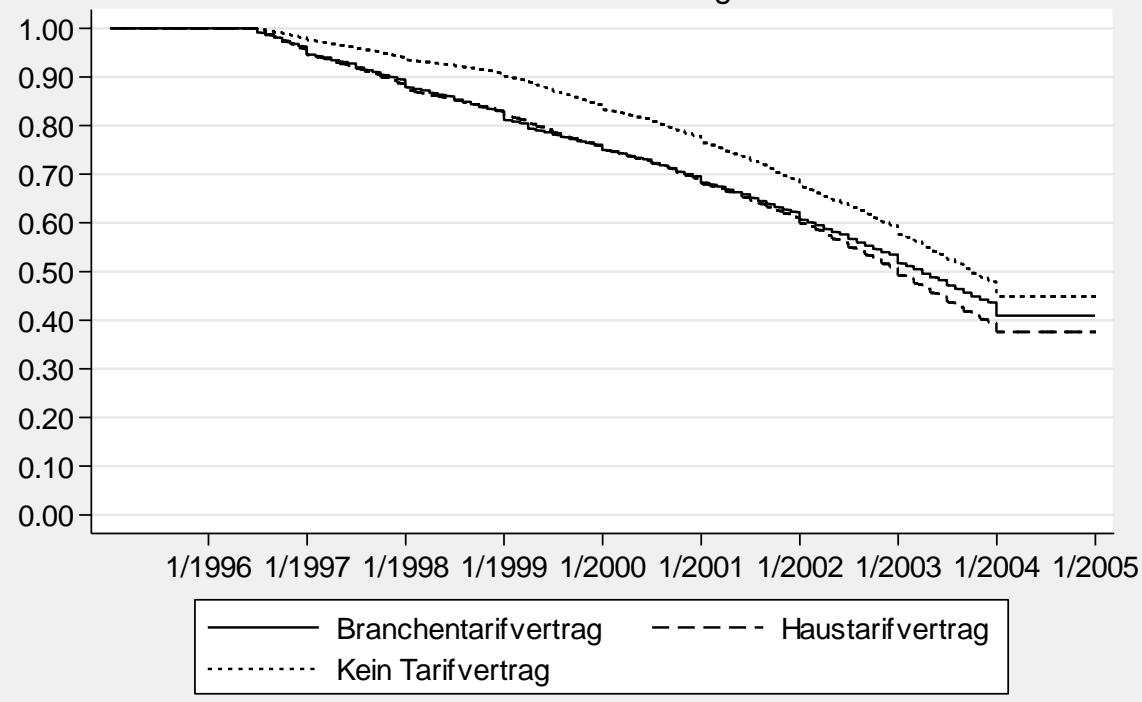

Quelle: LIAB, eigene Berechnungen

Mitbestimmung: Unterscheidet man zwischen Personen, die in einem Betrieb mit Betriebsoder Personalrat beschäftigt waren, und solchen in Betrieben ohne Arbeitnehmervertretung, wiederholt sich der zunächst unerwartete Befund, der sich für die Tarifvertragsvariable ergeben hatte. In Betrieben ohne Betriebs- oder Personalrat scheint die Wahrscheinlichkeit des Überganges aus der Erwerbstätigkeit geringer als in solchen mit einer Interessenvertretung der Beschäftigten, auch wenn die absoluten Differenzen über den Zeitverlauf hinweg gering sind. Diese Ungleichheit der Übergangsraten ist nach dem Log-rank- und dem Wilcoxon-Test auch hoch signifikant.

Kündigungsschutz: Auch für die Geltung des KSchG ergeben sich aus dem Vergleich keine positiven Effekte für den Verbleib Älterer in Erwerbstätigkeit. Personen aus Betrieben, für die der Kündigungsschutz galt, hatten eine signifikant höhere Wahrscheinlichkeit des Übergangs (vgl. Abb. 9 und Tab. 8). 
Abb. 8:

Kaplan-Meier-Schätzung - Mitbestimmung

Kaplan-Meier: Überleben in sv-pflichtiger Beschäftigung nach Mitbestimmung

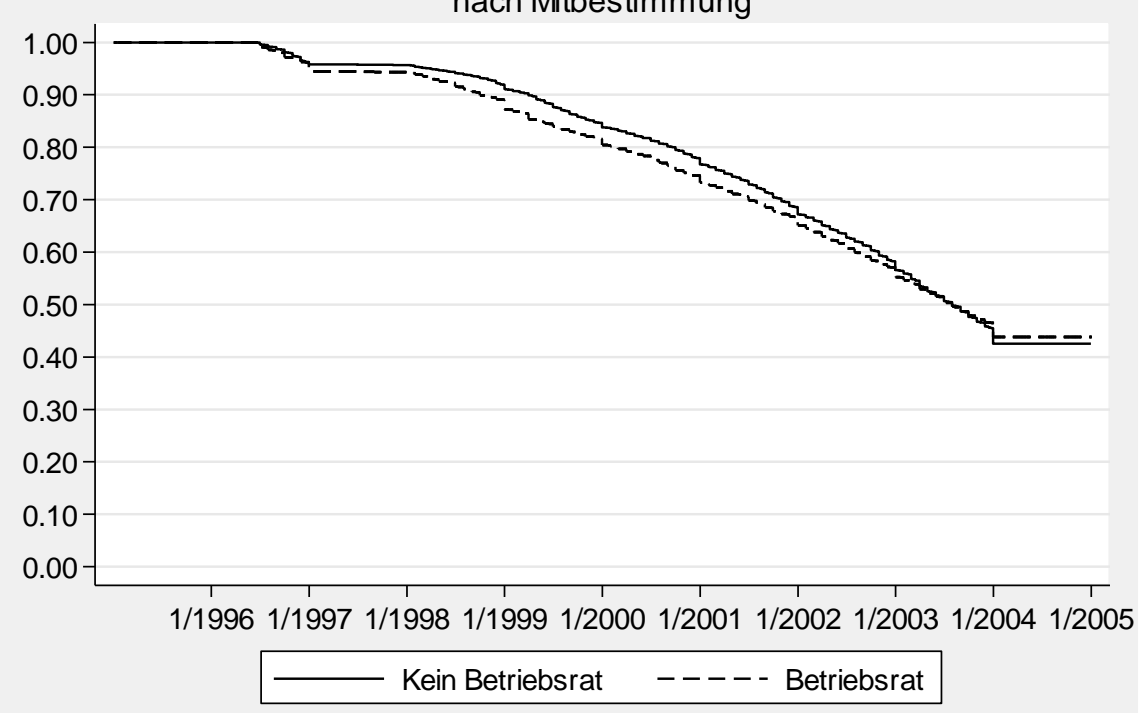

Quelle: LIAB, eigene Berechnungen

Abb. 9: $\quad$ Kaplan-Meier-Schätzung - Kündigungsschutz

Kaplan-Meier: Überleben in sv-pflichtiger Beschäftigung nach Geltung des KSchG

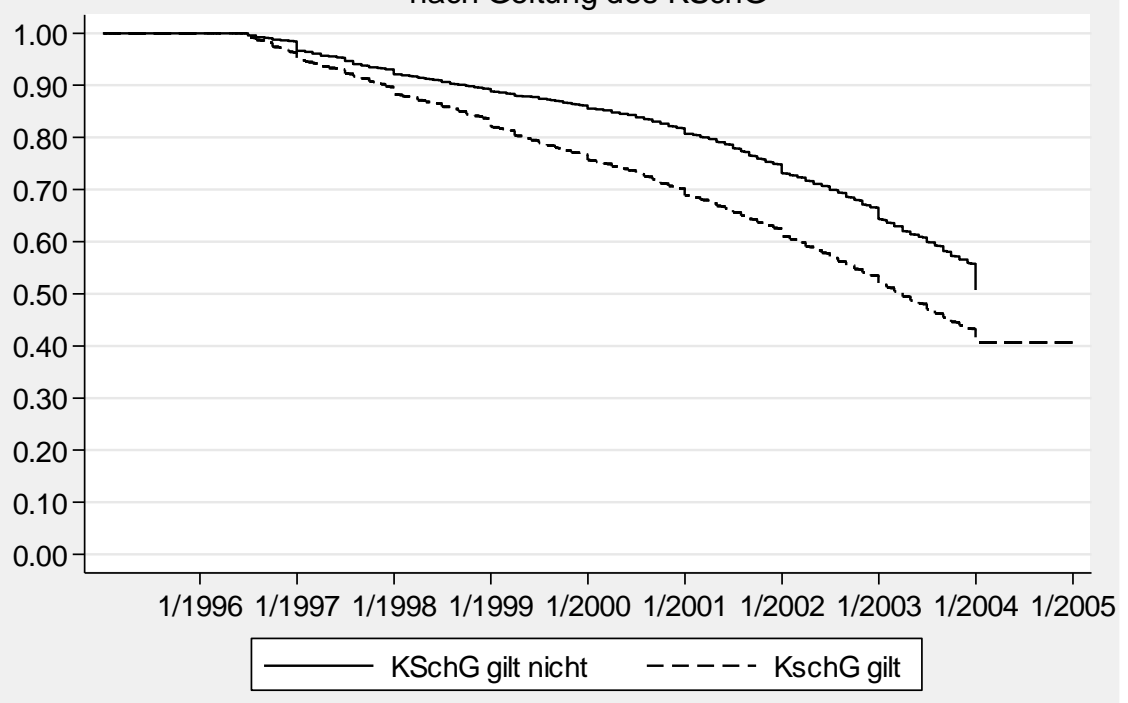

Quelle: LIAB, eigene Berechnungen 


\section{A 3.2.2 Anpassungskosten}

Anteil befristet Beschäftigter: Betriebe mit einem geringeren Anteil befristet Beschäftigter weisen eine höhere als die bei Gleichheit der Survivor-Funktionen zu erwartende Anzahl an Austritten auf. Wieder zeigen die beiden Tests daher signifikante Unterschiede für die hier zusammengefassten Kategorien des Anteils befristet Beschäftigter (vgl. Abb. 10 und Tab. ). Damit deutet sich an, dass die Weiterbeschäftigung Älterer auch von personalpolitischen Alternativen beeinflusst wird. Statt der Entlassung Älterer verfolgen Betrieb andere, möglicherweise kostengünstigere Anpassungsstrategien an eine geänderte Nachfrage nach Arbeitskräften.

Abb. 10: Kaplan-Meier-Schätzung - Anteil befristet Beschäftigter

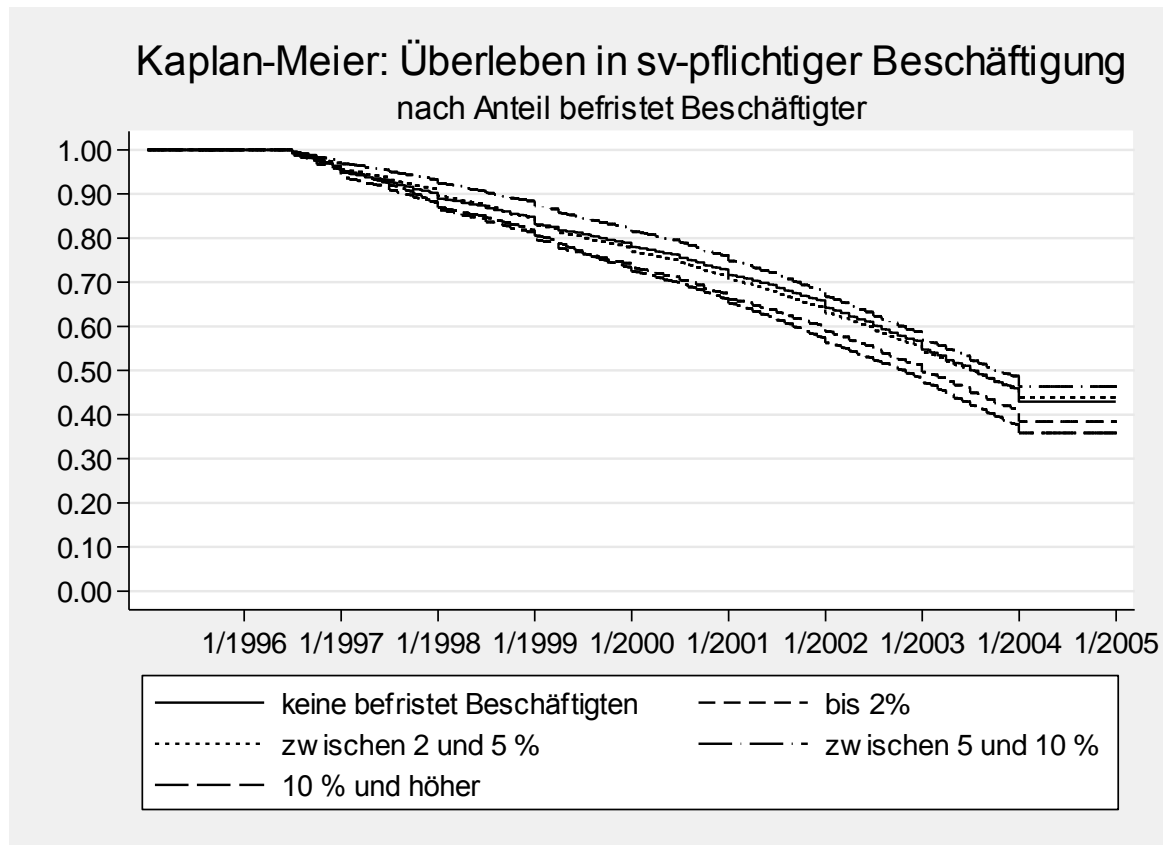

Quelle: LIAB, eigene Berechnungen

\section{A 3.2.3 Betriebliche Altersstruktur}

Anteil der Bis-30-Jährigen: Für die These, dass sich die ältere Belegschaft von Jüngeren aus dem Betrieb und mangels Wiederbeschäftigungsmöglichkeiten auch aus der Erwerbstätigkeit drängen lässt, findet sich in den deskriptiven Ergebnissen kein Anhaltspunkt. Gerade in Betrieben mit hohem Anteil an jungen Kollegen bleiben die beobachteten Austrittsereignisse hinter den erwarteten zurïck. Die in Tab. dargestellten Tests auf Gleichheit der Austrittsprozesse für hypothetische Personengruppen weist einen hochsignifikanten Unterschied nach. 
Abb. 11: Kaplan-Meier-Schätzung - Anteil bis-30-jähriger Beschäftigter

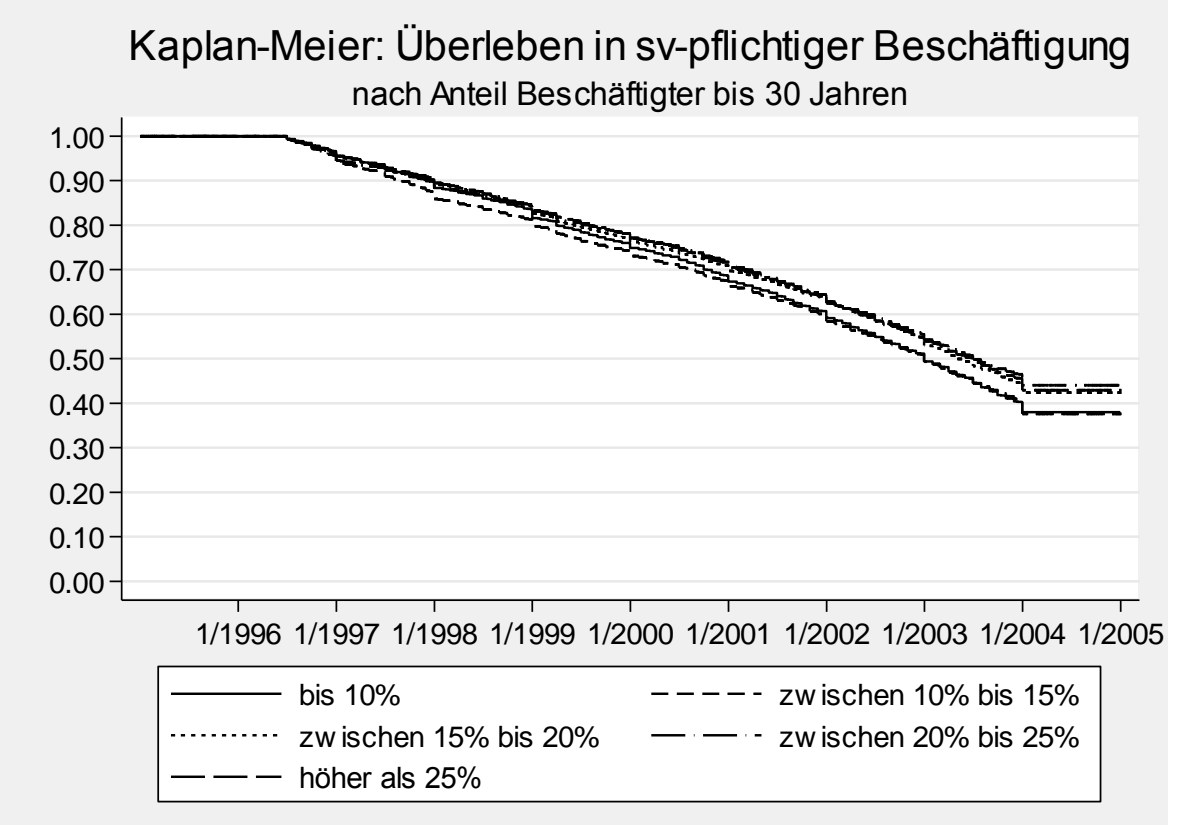

Quelle: LIAB, eigene Berechnungen

\section{A 3.2.4 Technischer Wandel}

Investitionen in Kommunikationstechnik: Auch die Vermutung eines altersverzerrenden technischen Fortschritts zuungunsten Älterer lässt sich, zumindest was den kurzfristigen Effekt von Investitionen in EDV angeht, nicht erhärten: Für Personen, die im Zeitablauf ununterbrochen in einem Betrieb beschäftigt waren, der in Kommunikationstechnik investierte, liegt die

Abb. 12: Kaplan-Meier-Schätzung - Investitionen in Kommunikationstechnik

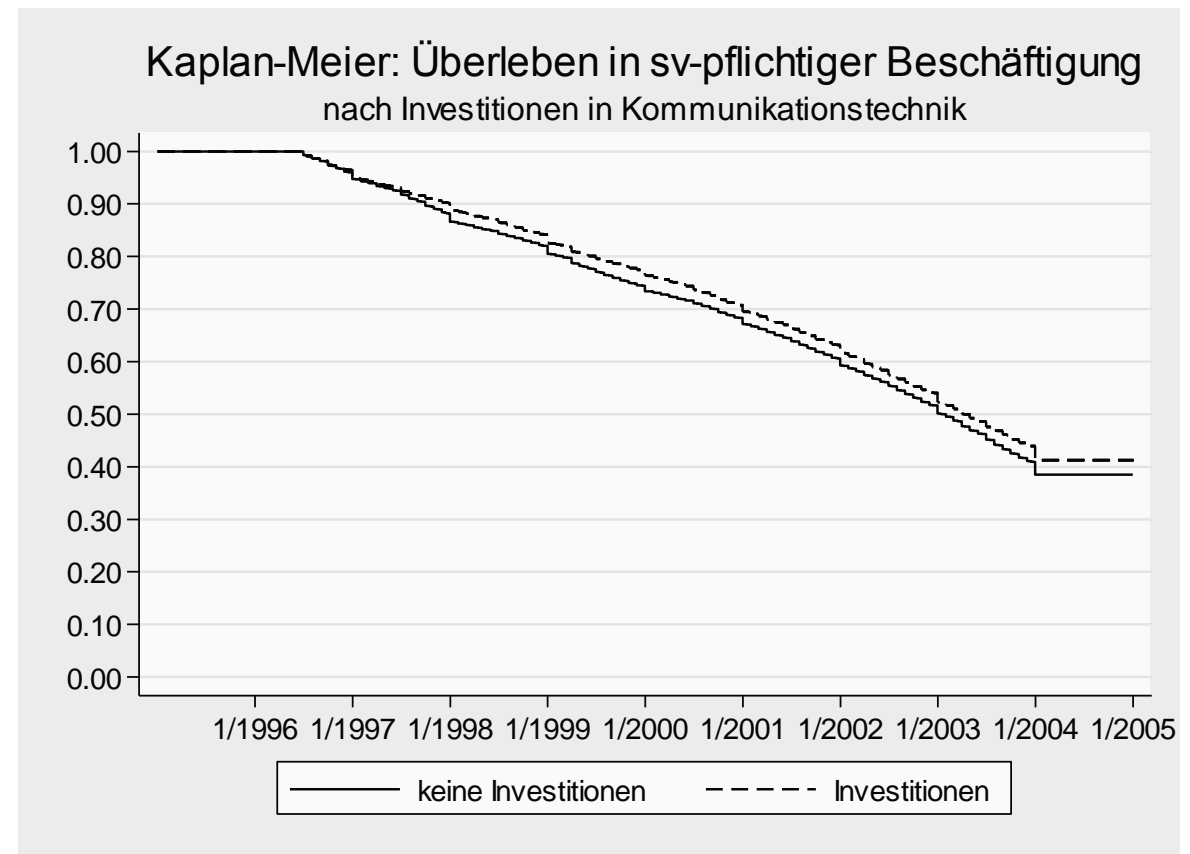

Quelle: LIAB, eigene Berechnungen 
Survivor-Funktion im gesamten Beobachtungszeitraum über der der Beschäftigten nichtinvestierender Betriebe. Die Tests in Tab. bestätigen das Ergebnis.

\section{A 3.2.5 Betriebsgröße}

Betriebsgröße: Auch für die Betriebsgröße finden sich Unterschiede im Austrittsverhalten. Abb. 13 mit Survivor-Funktionen für verschiedene Betriebsgrößen und Tab. mit den dazugehörigen Tests weisen darauf hin, dass Beschäftigung in einem kleinen Betrieb mit höheren Verbleiberaten einhergeht. Die Ergebnisse sind nach beiden angeführten Tests signifikant.

Abb. 13: $\quad$ Kaplan-Meier-Schätzung - Betriebsgröße in Anzahl Beschäftigten

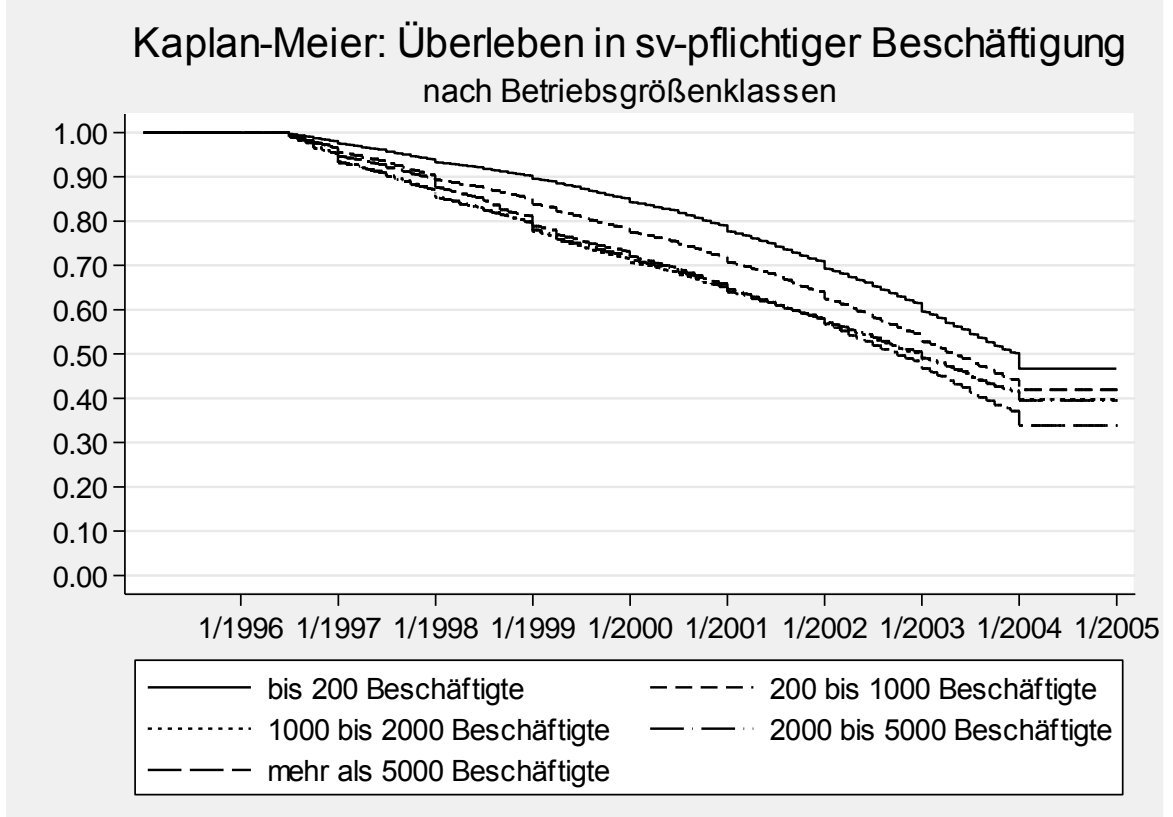

Quelle: LIAB, eigene Berechnungen

\section{A 3.3 Erwerbsaustritt: Zusammenfassung der deskriptiven Ergebnis- se}

Schon in der univariaten Analyse lassen sich deutliche Unterschiede im Austrittsverhalten feststellen. Zwar sind die hier vorgestellten Ergebnisse nicht dazu geeignet, als Effekt der jeweiligen erklärenden Variablen interpretiert zu werden, doch die jeweils hoch signifikanten Unterschiede im Vergleich der Austrittsprozesse deuten darauf hin, dass sich die in diesem Kapitel aufgeführten erklärenden Variablen als einflussreich bei der Erklärung des individuellen Austrittsereignisses erweisen. Zentrale Hypothesen des zweiten Kapitels wie der Einfluss neuer Technologien, die Bedeutung von Anpassungskosten und der Altersstruktur konnten untermauert werden. Dagegen sprechen die Ergebnisse für die institutionellen Rahmenbedingungen des Betriebes bislang nicht für die oben beschrieben Hypothesen.

Um die Wirkungen der einzelnen konkurrierenden Variablen zu schätzen, schließt sich eine multivariate Analyse an. Das dazu verwendete Cox proportional Hazardratenmodell wird da- 
her im nächsten Kapitel beschrieben, bevor die Darstellung und Diskussion seiner Ergebnisse folgt.

\section{A 4 Mikroökonometrischer Ansatz: Verweildaueranalyse zur Modellierung des Erwerbsaustritts}

Zur empirischen Analyse von Fragestellungen, die den Verbleib in oder den Übergang zwischen Zuständen zum Gegenstand haben, hat sich in den vergangenen Jahrzehnten ein ganzes Gebiet von Auswertungsmethoden entwickelt. Diese Verfahren der Verweildaueranalyse haben, nachdem sie zunächst meist für medizinische Fragestellungen verwendet wurden, Einzug auch in die empirische wirtschaftswissenschaftliche Forschung gehalten. ${ }^{54}$ Mittels solcher Verfahren werden Spelldaten analysiert und Bestimmungsgründe für Statuswechsel im zeitlichen Verlauf quantifiziert. Im Folgenden soll ein kurzer Überblick über die gängigen ökonometrischen Methoden der Verweildaueranalyse gegeben und dabei die Verwendung des Cox Proportional Hazardratenmodells begründet werden.

\section{A 4.1 Modellansatz}

Zentrales Ereignis ist der Übergang aus dem Erwerbsleben. Der Zeitpunkt dieses Übergangs wird durch die Variable T ausgedrückt. T stellt dabei eine stetige Zufallsvariable dar, deren Verteilung die Dichtefunktion $f(t)$ und die Verteilungsfunktion $F(t)=P(T \leq t)$ beschreiben. Die Verteilungsfunktion gibt die Wahrscheinlichkeit an, dass das Ereignis des Austritts aus dem Erwerbsleben bis zum Zeitpunkt $t$ statt findet. Neben der Verteilungs- und Dichtefunktion des Übergangszeitpunktes haben sich andere Konzepte zur Beschreibung des Prozesses eingebürgert. Eng angelehnt an die Verteilungsfunktion $F(t)$ ist die Survivor-Funktion $S(t)$. Sie gibt die Wahrscheinlichkeit an, dass eine Person zum Zeitpunkt $t$ den Übergang noch nicht vollzogen hat, sich - im Fall dieser Untersuchung - noch in Beschäftigung befindet. $S(t)$ ergibt einfach als

$$
S(t)=1-F(t)=P(T>t) .
$$

Die Survivor-Funktion ist eine in $\mathrm{t}$ monoton abnehmende Funktion, die zum Zeitpunkt $\mathrm{t}=0$ den Wert 1 annimmt und auf 0 absinkt, sobald alle Individuen den Statusübergang vollzogen haben. Die durchschnittliche Verweildauer im Ausgangszustand lässt sich als das Integral über die Survivor-Funktion $\int_{0}^{\infty} S(u) d u$ darstellen.

\footnotetext{
${ }^{54}$ Aus diesem Grund begegnet man neben der Bezeichnung Verweildaueranalyse in der Literatur auch dem synonymen Begriff der Überlebensanalyse. Die Ereignisanalyse - obwohl gelegentlich auch verwendet - bezeichnet dagegen alle Verfahren, die das Eintreten definierter Ereignisse erklären sollen, so z.B. auch Zähldatenmodelle. Anwendungsbeispiele sind neben dem Verbleib in der Arbeitslosigkeit auch Analysen der Streikdauer oder bevölkerungsökonomische Fragestellungen wie Untersuchungen des Zeitpunktes der Geburt des ersten Kindes.
} 
Eine weitere gängige Darstellungsweise ist die Hazardrate $\theta(t)$. Sie beschreibt die bedingte Dichte des Übergangs (hier: aus der Beschäftigung), gegeben die Person hat sich bis zum Zeitpunkt $t$ in der „Risikogruppe“ (hier: der Erwerbstätigen) befunden. Die Hazardrate lässt sich darstellen als

$$
\theta(t)=\lim _{\Delta t \rightarrow 0} \frac{P(t \leq T<t+\Delta t \mid T \geq t)}{\Delta t} .
$$

Der Ausdruck kann man demnach interpretieren als eine bedingte Dichte der Übergangs(zeitpunkts-)variablen $\mathrm{T}$ an der Stelle $\mathrm{t}$, gegeben, dass bis zum Zeitpunkt $\mathrm{t}$ dieses Ereignis noch nicht stattgefunden hat. Die zentrale Bedeutung des Konzepts der Hazardrate ergibt sich daraus, dass Verweildaueranalysen trotz ihrer sperrigen Interpretation die Erklärung der bedingten Hazardrate $\theta(t \mid x)$ zum Ziel haben, während in anderen regressionsanalytischen Methoden das zentrale Auswertungsinteresse meist in der Bestimmung von $E(T \mid x)$ liegt.

\section{A 4.2 Spezifikation des Hazardratenmodells}

Im Folgenden soll anhand einer Charakterisierung dieser gängigen Methode der Verweildaueranalyse die Wahl des Cox Proportional Hazardratenmodells erläutert werden, indem Vorzüge und Probleme des Schätzansatzes dargestellt werden.

Die zentrale Annahme aller proportionalen Hazardratenmodelle ${ }^{55}$ besagt, dass sich die individuelle Hazardrate in zwei Faktoren separieren lässt:

$$
\theta(t)=\theta_{0}(t, \alpha) \phi(x, \beta)
$$

Dabei bezeichnet $\theta_{0}$ die Baseline-Hazardrate, die unabhängig von den untersuchten Individuen ist und ein zeitabhängiges Maß für das Grundrisiko des Übergangs darstellt. Sie hängt ausschließlich vom Zeitindex $\mathrm{t}$ und gegebenenfalls den Parametern $\alpha$ einer zuvor unterstellten funktionalen Form der Baseline-Hazardrate ab. Die Interpretation des (zeitlichen Verlaufs) dieses Terms ähnelt der des Absolutterms in der linearen Regression: Nur bei geeigneter Skalierung der Regressoren kann sein Wert Gegenstand einer sinnvollen Interpretation sein. ${ }^{56}$ Was die funktionalen Annahmen bezüglich der Baseline-Hazardrate angeht, so hat sich neben der polynomialen Ausgestaltung der funktionalen Form insbesondere eine nichtparametrische Schätzung etabliert.

\footnotetext{
${ }^{55}$ Die alternative Großgruppe von Verweildauermodellen, die „Accelerated failure time“ Modelle, unterstellen, dass die Änderung einer Kovariaten sich in einer proportionalen Änderung der erwarteten Übergangszeit niederschlägt. Solche Modelle unterscheiden sich vom hier gewählten PH-Modell vor allem darin, dass sie nur voll parametrisch geschätzt werden können.
}

${ }^{56} \mathrm{Vgl}$. Jenkins (2005). 
Gewöhnlich wird der individuelle Teil dieser Hazardratenfunktion als $\phi(x, \beta)=\exp \left(x^{\prime} \beta\right)$ parametrisiert. Interpretatorisch vorteilhaft an proportionalen Hazardraten-(PH)-Modellen ist die Unabhängigkeit des individuellen Parts der Hazardrate vom Zeitindex. So lassen sich die zentralen Ergebnisse der Schätzung, die Parameter $\beta$, zeitunabhängig interpretieren: Die PHAnnahme impliziert nämlich, dass sich absolute Unterschiede in der Ausprägungen von X zu jedem Zeitpunkt $t=\bar{t}$ als proportionale Änderung der individuellen Hazardrate auffassen lassen. Die Relation zwischen zwei individuellen Hazardraten lautet daher

$$
\frac{\theta\left(\bar{t}, x_{i}\right)}{\theta\left(\bar{t}, x_{j}\right)}=\exp \left[\beta^{\prime} x_{i}-\beta^{\prime} x_{j}\right] .
$$

Für zwei Individuen mit bis auf $\mathrm{x}_{\mathrm{k}}$ identischen Kovariaten, also $\mathrm{x}_{\mathrm{im}}=\mathrm{x}_{\mathrm{jm}}$ für alle $m \in$ $\{1, \ldots K \bigvee\}\}$ gilt für den Koeffizienten $\beta_{k}$, dass

$$
\frac{\theta\left(\bar{t}, x_{i}\right)}{\theta\left(\bar{t}, x_{j}\right)}=\exp \left[\beta_{k}\left(x_{i k}-x_{j k}\right)\right] .
$$

Der Term $\exp \beta_{k}$, dessen Schätzung sich in den folgenden Ergebnistabellen wiederfindet, wird daher auch als „hazard ratio“ bezeichnet und kennzeichnet die proportionale Änderung der Hazardrate, die mit einer Änderung der zugehörigen Hintergrundvariable um eine Einheit einhergeht. Aufgrund der Eigenschaft von $\beta_{k}=\partial \log \theta(t, x) / \partial x_{k}$ wird der Parameter auch als Elastizität der Hazardrate in Bezug auf die logarithmierte Variable $x_{k} \equiv \log z_{k}$ interpretiert.

Weiterhin impliziert die PH-Annahme, dass für Personen mit identischen Kovariaten $x=\bar{x}$ aber unterschiedlichen Verweildauern die Relation der Hazardraten einzig von den BaselineHazardraten zu den verschiedenen Zeitpunkten u und t abhängt:

$$
\frac{\theta(t, \bar{x})}{\theta(u, \bar{x})}=\frac{\theta_{0}(t)}{\theta_{0}(u)} .
$$

Je nach Datengrundlage unterscheidet man zwischen stetigen und diskreten Modellen. Die meisten sozialwissenschaftlichen Daten liegen aufgrund der Erhebungsweise nur in diskreter Ausprägung vor. So werden Übergangszeitpunkte nur in groben Zeitintervallen berichtet. Liegen die Daten zur Verweildauer trotz grundsätzlich stetiger Natur nur in diskreter Form vor oder können die Daten von vornherein nur diskrete Ausprägungen der Verweildauer annehmen, bietet es sich an, das oben beschriebene Modell auch für diese Datengrundlage abzuwandeln. In solchen diskreten Modellen wird dann nicht wie im stetigen Fall die bedingte Dichte des Übergangs zu einem Zeitpunkt, sondern die bedingte Wahrscheinlichkeit des Übergangs innerhalb eines Zeitraumes geschätzt.

Im vorliegenden Fall des LIAB ist die Art der Gruppierung je nach Variable unterschiedlich. Die Betriebsdaten sind aufgrund des jährlichen Zyklus nur in diesem Turnus erhoben. Daten 
zum Erwerbsverlauf und damit auch die Verweildauervariable $t$ sind aufgrund der tagesgenauen Erfassung von Beginn und Ende einer sozialversicherungspflichtigen Beschäftigung allerdings nahezu stetig. Für die Analyse wurde daher eine Methode zur Untersuchung stetiger Verweildauerdaten verwendet.

\section{A 4.3 Das Cox Proportional Hazardratenmodell}

Parametrische Hazardratenmodelle haben den Vorteil, dass sie mittels Maximum Likelihood(ML)-Verfahren einfach zu schätzen sind. Allerdings liefern sie inkonsistente Ergebnisse für den Fall einer Fehlspezifikation bezüglich der zugrunde gelegten parametrischen Form der Hazardrate. Wie in Kapitel A 1 angeführt, kann ein solcher idealer Verlauf der Hazardrate für das Austrittsverhalten nicht von vornherein angenommen werden. Es ist daher ein besonderer Vorzug des verwendeten Cox Proportional Hazardratenmodells, dass es ohne solche strengen Annahmen auskommt. Stattdessen ermittelt man die Baseline-Hazardrate in diesem Modellrahmen nichtparametrisch. Es wird daher auch als semiparametrisch bezeichnet und hat sich zu einer Standardmethode in der Verweildaueranalyse entwickelt.

\section{A 4.3.1 Partial-Likelihood-Schätzung ${ }^{57}$}

Anders als bei parametrischen Schätzverfahren der Verweildaueranalyse verwendet das CoxModell keine ML-Schätzung zur Bestimmung der Koeffizienten $\beta$. Stattdessen wird für jeden Zeitpunkt eines Übergangs $\mathrm{t}_{\mathrm{j}}$ für die bis dahin verbliebene Risikomenge $R\left(t_{j}\right)$ die Partial Likelihood (PL) maximiert. Die Methode soll im Folgenden kurz erläutert werden.

Zunächst bezeichnen $\mathrm{t}_{1}<\mathrm{t}_{2}<\mathrm{t}_{3}<\ldots<\mathrm{t}_{\mathrm{j}}<\ldots<\mathrm{t}_{\mathrm{k}}$ die geordneten Übergangsdauern bei einem Beobachtungsumfang von $N \geq k$. Als Risikomenge $R\left(t_{j}\right)$ wird die Menge an Individuen definiert, die direkt vor jedem Zeitpunkt $t_{j}$ den Statuswechsel noch nicht vollzogen hat und daher noch dem Risiko des Übergangs ausgesetzt ist. Mit $D\left(t_{j}\right)$ bezeichnet man die Menge der Individuen, die zum Zeitpunkt $t_{j}$ aus der Risikomenge austritt. Die Anzahl der Individuen, für die in $t_{j}$ das untersuchte Ereignis der Renteneintritt stattfindet, ist $d_{j}$. Es können demnach mehrere Personen gleichzeitig übertreten.

Die Wahrscheinlichkeit, dass genau der Spell der Person j derjenige ist, der aus der Risikomenge fällt, ergibt sich dann als die einzelne bedingte Wahrscheinlichkeit des Übergangs dividiert durch die Summe der Wahrscheinlichkeiten, dass die anderen Individuen der Risikomenge den Wechsel nicht vollziehen:

$$
P\left(T_{j}=t_{j} \mid R\left(t_{j}\right)\right) \quad=\frac{P\left(T_{j}=t_{j} \mid T_{j} \geq t_{j}\right)}{\sum_{l \in R\left(t_{j}\right)} P\left(T_{l}=t_{l} \mid T_{l} \geq t_{j}\right)}
$$

\footnotetext{
${ }^{57}$ Darstellung angelehnt an Cameron und Trivedi (2005) S. 594ff.
} 


$$
=\frac{\theta_{j}\left(t_{j} \mid x_{j}, \beta\right)}{\sum_{l \in R\left(t_{j}\right)} \theta_{l}\left(t_{j} \mid x_{l}, \beta\right)}
$$

Aufgrund der PH-Annahme lässt sich der Term um den Ausdruck der Baseline-Hazardrate vereinfachen, wobei $\phi\left(x_{j}, \beta\right)$ wieder als $\exp \left(x_{j}^{\prime} \beta\right)$ spezifiziert wird. Es ergibt sich:

$$
P\left(T_{j}=t_{j} \mid R\left(t_{j}\right)\right) \quad=\frac{\phi\left(x_{j}, \beta\right)}{\sum_{l \in R\left(t_{j}\right)} \phi\left(x_{l}, \beta\right)} .
$$

Damit lässt sich der Koeffizientenvektor $\beta$ aufgrund der PH-Annahme schätzen. Erschwert wird die Analyse bei gruppierten Spelldaten und Massendaten typischerweise durch gleichzeitig auftretende oder gleichzeitig beobachtete Übergänge. Nach Breslow und Peto (vgl. Cox und Oakes (1984)) kann die oben bestimmte bedingte Wahrscheinlichkeit des Übergangs von Individuum $\mathrm{j}$ dann näherungsweise dargestellt werden als:

$$
P\left(T_{j}=t_{j} \mid j \in R\left(t_{j}\right)\right) \cong \frac{\prod_{m \in D\left(t_{j}\right)} \phi\left(x_{m}, \beta\right)}{\left[\sum_{l \in R\left(t_{j}\right)} \phi\left(x_{l}, \beta\right)\right]^{d_{j}}} .
$$

Cox hat das gemeinsame Produkt dieser Wahrscheinlichkeiten des Austritts genau der Person(en) aus der Risikogruppe als PL-Funktion definiert (Vgl. Cameron und Trivedi (2005), S. 595 f.). Sie ergibt sich als

$L_{p}(\beta)$

$$
\cong \prod_{j=1}^{k} \frac{\prod_{m \in D\left(t_{j}\right)} \phi\left(x_{m}, \beta\right)}{\left[\sum_{l \in R\left(t_{j}\right)} \phi\left(x_{l}, \beta\right)\right]^{d_{j}}} .
$$

Die in $\phi\left(x_{m}, \beta\right)$ spezifizierte PL-Funktion lässt sich logarithmiert bezüglich der Parameter $\beta$ numerisch maximieren. Nach Schätzung der Koeffizienten lässt sich die Baseline-Hazardrate nichtparametrisch ähnlich dem Kaplan-Meier-Verfahren schätzen.

Aufgrund der PH-Annahme ließ sich der Term der Baseline-Hazardrate aus der PL-Funktion herauskürzen. Der Schätzansatz beruht daher nicht auf vollständiger Information und ist daher nur ein Limited Information Maximum-Likelihood-Schätzer. Er zog damit die Kritik von Forschern auf sich, die der Full Information Maximum-Likelihood-Spezifikation von parametrischen Verweildauermodellen den Vorzug gaben. Allerdings konnte gezeigt werden, dass die PL-Schätzung konsistent und asymptotisch normalverteilt ist (Andersen et al. (1993)). Zudem ist die Schätzung asymptotisch äquivalent mit der Maximum-Likelihood-Schätzung der vollständigen Likelihood-Funktion (Bailey 1984). 
Wie in der Beschreibung der PH-Modelle allgemein schon erwähnt, sind die exponierten Koeffizienten zu interpretieren als Faktor, um den sich die Hazardrate verändert, wenn sich der Wert des zugehörigen Regressors um eine Einheit erhöht. Zudem muss erwähnt sein, dass das Cox Proportional Hazardratenmodell kein Absolutglied schätzt, da dieses nicht identifiziert ist (vgl. Clewes et al. (2004), S. 123).

Neben den zentralen empirischen Ergebnissen lassen sich einige Tests durchführen, die in der Literatur unter dem Namen „Test der Proportional Hazard Annahme“ aufgeführt werden. Allerdings leitet diese Bezeichnung in die Irre, da sie statt die dem PH-Modell zugrunde liegende Annahme zu überprüfen, Tests der Modellparametrisierung darstellen und untersuchen, ob die Einbeziehung eines höheren Polynoms der Regressoren zusätzliche Erklärungskraft bietet.

\section{A 4.3.2 Zensierung}

$\mathrm{Zu}$ erläutern bleibt noch, wie eine eingeschränkte Beobachtbarkeit der Variablen auf die Schätzung wirkt: Zunächst sind drei Formen zeitlicher Zensierung vorstellbar und aufgrund des großen Datensatzes und der Stichprobenauswahl auch sicher anzutreffen. Personen, die dem Risiko des Übergangs bereits vor Beginn des Beobachtungszeitraums ausgesetzt waren, liefern linkszensierte Beobachtungen, da keine Angaben über die Hintergrundsvariablen vor diesem Zeitpunkt vorhanden sind. Bei den verwendeten Daten liegt sogar eine Linksstutzung vor, da Personen der untersuchten Geburtsjahrgänge, die vor ihrem 50.Geburtstag das Erwerbsleben verlassen hatten, nicht in die Stichprobe eingehen und nicht als Übergänge gelten. Als Rechtszensierung bezeichnet man solchen Fälle, die zum Ende des Beobachtungszeitraumes den Übergang aus dem Erwerbsleben noch nicht vollzogen hatten. Weder ist bekannt, zu welchem Zeitpunkt sie den Austritt vornehmen, noch wie sich die Hintergrundvariablen in der Zeit zwischen Ende des Beobachtungszeitraums und Austritt entwickeln. Einzig, dass die Person bis dahin in der Risikogruppe verblieben ist und sie erst nach diesem Zeitpunkt verlassen wird, ist sicher. Dies trifft auf rund 30\% der Personen der Stichprobe zu. Eine weitere Art der Zensierung besteht darin, dass Fälle nur mit Unterbrechungen beobachtet werden konnten, so genannte Intervallzensierung. Insbesondere dann, wenn Panelbetriebe in einzelnen Jahren nicht an der Umfrage teilgenommen haben oder Personen für einige Zeit ihre Beschäftigung in einem Panelbetrieb unterbrochen haben, ergeben sich zeitliche Lücken in diesen Angaben.

Keine der aufgeführten Formen der Zensierung stellt das verwendeten Cox proportional Hazardratenmodell in Frage (vgl. Clewes et al. (2004), S. 29 ff.), einzig die zu Grunde liegende Grundgesamtheit muss angepasst werden. Für die Interpretation der Ergebnisse bleibt nämlich die Linksstutzung zu beachten: dass sich die Aussagen auf ältere Arbeitnehmer beziehen, also Personen, die bis zu einem Alter von mindestens 50 Jahren noch einer Erwerbstätigkeit nachgegangen sind. Auch können Erwerbsverläufe von Personen untersucht werden, für die nicht durchgängig Betriebsinformationen vorhanden sind oder die über das Ende der Beobachtungszeit im Erwerbsleben verbleiben. 


\section{A 4.4 Kontrolle unbeobachteter Heterogenität}

In den verwendeten Daten lassen sich zwei Arten von unbeobachteter, für das Austrittsereignis relevanter Heterogenität unterscheiden: betriebliche und persönliche Variablen, die im Datensatz nicht erhoben sind. Ihre Korrelation mit einer der im Modell berücksichtigten Variablen führt zu einer Verzerrung der geschätzten Koeffizienten und damit der ermittelten Hazard ratio.

In der folgenden Cox-Regression ließe sich dabei für betriebsspezifische unbeobachtete Heterogenität kontrollieren, indem eine Baseline-Hazardrate $\theta_{\mathrm{j} 0}$ für jeden Betrieb geschätzt würde und somit für jede Kombination aus Betrieb und Austrittszeitpunkt ein fixer Term die Einflüsse solcher betrieblicher unbeobachteter Heterogenität erfasst würde. Die Hazardrate ließe sich dann wieder als multiplikative Verknüpfung dieser Baseline-Hazardrate mit dem unveränderten individualisierten Term

$$
\theta_{j}(t)=\theta_{0 j}(t) \phi(x, \beta)
$$

darstellen. Das Vorgehen kann als fixed-effects-Schätzer interpretiert werden, wie sie bei Paneluntersuchungen von linearen Zusammenhängen Verwendung finden. Allerdings bezahlt man bei dieser Methode die Kontrolle betrieblicher Einflüsse damit, dass einige beobachtbare Variable außen vor bleiben müssen. So sind in diesem Modell die zu schätzenden Koeffizienten von zeitlich konstanten Betriebsvariablen nicht identifiziert, sodass das Modell um diese Einflussfaktoren beschnitten werden müsste. Aus diesem Grund und weil die gewünschte Spezifikation des Modells unter Kontrolle unbeobachteter Betriebsheterogenität keine Konvergenz erbrachte, musste von der Schätzung eines solchen Modells Abstand genommen werden. Eine Anwendung solcher Schätzmethoden auf den LIAB findet sich in der Arbeit von Boockmann und Steffes (2005). Eine methodische Abhandlung über das stratifizierte Modell findet sich bei Ridder und Tunali (1999).

\section{A 5 Mikroökonometrische Ergebnisse: Determinanten des Er- werbsaustritts}

Im Folgenden werden die multivariaten Ergebnisse für die Erklärung des Erwerbsaustritts mittels einer Cox-Regression dargestellt und diskutiert. Dabei werden jeweils getrennte Analysen für Frauen und Männer vorgestellt und die Personen aus den alten und neuen Bundesländern jeweils separat behandelt. Diese Unterteilungen scheinen angebracht, da sich einerseits das Rentenzugangsverhalten von Frauen und Männern aufgrund unterschiedlicher Erwerbsverläufe und Rentenzugangsformen unterscheiden. Ebenso lässt sich - trotz der inzwischen langjährigen staatlichen Einheit - die getrennte Betrachtung ost- und westdeutscher Versicherter rechtfertigen mit unterschiedlichen Anwartschaften der beiden Bevölkerungsgruppen und unterschiedlichen makroökonomischen Umständen der beiden Landesteile. Auch

die uneinheitliche Verfügbarkeit von Daten über den Erwerbsverlauf vor 1990 macht eine 
getrennte Betrachtung von Ost- und Westdeutschland notwendig und erlaubt so eine angemessene Analyse.

Zunächst werden die Ergebnistabellen präsentiert und danach nach Variablenblöcken interpretiert. Die folgenden Ergebnisse beziehen sich auf ein nicht-stratifiziertes Cox proportional Hazardratenmodell. Versuche, ein solches Modell unter Kontrolle unbeobachteter Betriebsheterogenität zu schätzen, schlugen fehl. Es konnte daher nicht für betriebsspezifische Heterogenität kontrolliert werden. Die gerade auf Betriebsebene tief gehende Datenbasis lässt allerdings erwarten, dass bestimmende Einflussgrößen, die den beschäftigenden Betrieb betreffen, berücksichtigt wurden und daher verzerrende Effekte einer möglichen Fehlspezifikation aufgrund ausgelassener Variablen die hier gewonnenen Aussagen nicht in Frage stellen. Zudem haftet einer stratifizierten Modellspezifikation der Makel an, dass Koeffizienten zeitlich konstanter Betriebsvariablen in diesem Fall nicht identifiziert sind. Da aber der Schwerpunkt dieser Arbeit auf der Frage liegt, wie betriebliche Einflüsse wirken, scheinen die Vorteile eines unstratifizierten Modells hier zu überwiegen.

Tab. stellt die Schätzung der Hazard ratios der einzelnen Regressoren für Westdeutschland dar. Die zusammenfassenden Ergebnisse berichten von 199812 Personen, von denen 48149 nicht aus dem Erwerbsleben austreten. Der $\mathrm{Chi}^{2}$-Test deutet auf eine hohe Signifikanz des gewählten Modellansatzes. Ebenso sind auf den folgenden Seiten die Schätzungen für die westdeutschen Frauen der Geburtsjahrgänge 1945 und früher aufgeführt. Von den 88416 Frauen wurden 22333 zum Ende des Jahres 2003 als noch als erwerbstätig angesehen. Wieder erlaubt der hohe signifikante $\mathrm{Chi}^{2}$-Wert die Aussage, dass die Gesamtheit der gewählten Regressoren Einfluss auf den Austrittszeitpunkt ausübt. 
Tab. 9:

Cox proportional hazard rate- Schätzungen - Westdeutschland

\begin{tabular}{|c|c|c|c|c|}
\hline Männer West & \multicolumn{4}{|c|}{ Frauen West } \\
\hline $\begin{array}{l}\text { No. of subjects }= \\
\text { No. of failures }= \\
\text { Time at risk }= \\
\text { Log likelihood }= \\
\text { Number of obs }= \\
\text { LR chi2 } 2(117)= \\
\text { Prob }>\text { chi2 }= \\
\end{array}$ & $\begin{array}{l}\text { No. of subje } \\
\text { No. of failur } \\
\text { Time at risk } \\
\text { Log likelihoc } \\
\text { Number of c } \\
\text { LR chi2(137 } \\
\text { Prob }>\text { chi2 } \\
\end{array}$ & & & $\begin{array}{r}88416 \\
22333 \\
524667479 \\
-220586,93 \\
198363 \\
34649,92 \\
0,0000 \\
\end{array}$ \\
\hline \multirow[b]{2}{*}{ Personenvariablen } & \multicolumn{2}{|c|}{ Männer West } & \multicolumn{2}{|c|}{$\begin{array}{ll}\text { Frauen West } \\
\end{array}$} \\
\hline & h - ratio & $P>|z|$ & $\mathrm{h}$ - ratio & $P>|z|$ \\
\hline Alter (in Jahren) & 0,956 & 0,000 & 0,987 & 0,012 \\
\hline \multirow{2}{*}{$\begin{array}{l}\text { Freistellung in den vergangenen zwei Jahren } \\
\quad x(\text { Alter=59) }\end{array}$} & 5,911 & 0,000 & 7,741 & 0,000 \\
\hline & 0,414 & 0,000 & $\begin{array}{l}1,009 \\
0,189\end{array}$ & $\begin{array}{l}0,941 \\
0,000\end{array}$ \\
\hline$x($ Alter $=61)$ & \multirow{2}{*}{0,451} & \multirow{2}{*}{0,000} & 0,316 & 0,000 \\
\hline$x($ Alter=62) & & & 0,177 & 0,000 \\
\hline$x($ Alter=63) & \multirow{2}{*}{0,152} & \multirow{2}{*}{0,000} & 0,184 & 0,000 \\
\hline$x($ Alter=64) & & & 0,365 & 0,000 \\
\hline$x($ Alter $>64)$ & 0,156 & 0,000 & 0,109 & 0,000 \\
\hline Anzahl Freistellungen & 0,777 & 0,000 & 0,769 & 0,000 \\
\hline Anzahl Freistellungen² & 1,020 & 0,002 & 1,004 & 0,000 \\
\hline Eintritt ins Erwerbsleben & 0,99985 & 0,000 & 0,99981 & 0,000 \\
\hline Eintritt ins Erwerbsleben² & 1,00003 & 0,000 & 1,000067 & 0,000 \\
\hline Eintritt in den Betrieb & 0,99994 & 0,000 & 1,000041 & 0,029 \\
\hline Eintritt in den Betrieb² & 0,999994 & 0,000 & 0,99994 & 0,000 \\
\hline Deutsch & 1,057 & 0,094 & 1,013 & 0,815 \\
\hline$x($ Alter=59) & \multirow{2}{*}{1,069} & \multirow{2}{*}{0,123} & 0,808 & 0,057 \\
\hline$x($ Alter $=60)$ & & & 1,461 & 0,000 \\
\hline$x($ Alter $=61)$ & \multirow{2}{*}{1,107} & \multirow{2}{*}{0,040} & 1,001 & 0,995 \\
\hline$x($ Alter=62) & & & 0,898 & 0,335 \\
\hline$x($ Alter $=63)$ & \multirow{2}{*}{1,482} & \multirow{2}{*}{0,000} & 0,706 & 0,006 \\
\hline$x($ Alter $=64)$ & & & 0,556 & 0,000 \\
\hline$x($ Alter $>64)$ & 0,509 & 0,000 & 0,419 & 0,000 \\
\hline Berufliche Stellung & \multicolumn{4}{|c|}{ Referenzkategorie: Angestellte/r } \\
\hline Einfacher Arbeiter & 0,733 & 0,000 & 0,882 & 0,000 \\
\hline Facharbeiter & 0,818 & 0,000 & 0,870 & 0,028 \\
\hline Meister & 1,189 & 0,000 & 1,106 & 0,680 \\
\hline Teilzeit $<15$ Std./Woche & 0,313 & 0,000 & 0,370 & 0,000 \\
\hline Teilzeit >15 Std./Woche & 0,890 & 0,000 & 0,801 & 0,000 \\
\hline Schul-/Ausbildung & \multicolumn{4}{|c|}{ Referenzkategorie: Hochschulabschluss } \\
\hline Bis mittlere Reife ohne Berufsausbildung & 1,192 & 0,000 & 1,816 & 0,000 \\
\hline Bis mittlere Reife mit Berufsausbildung & 1,374 & 0,000 & 1,993 & 0,000 \\
\hline (Fach-) Hochschulreife ohne Berufsausbildung & 1,044 & 0,538 & 1,421 & 0,002 \\
\hline (Fach-) Hochschulreife mit Berufsausbildung & 1,184 & 0,000 & 1,909 & 0,000 \\
\hline Fachhochschulabschluss & 1,381 & 0,000 & 2,036 & 0,000 \\
\hline Tagesentgelt [in $€$ ] & 1,00036 & 0,000 & 1,00388 & 0,000 \\
\hline$x($ Alter $=59)$ & & & 1,00045 & 0,115 \\
\hline$x($ Alter $=60)$ & 0,99985 & 0,001 & 0,99953 & 0,010 \\
\hline$x($ Alter $=61)$ & 099965 & חمחת & 0,99924 & 0,002 \\
\hline$x($ Alter $=62)$ & 0,99965 & 0,000 & 1,00080 & 0,010 \\
\hline$x($ Alter $=63)$ & & & 1,00073 & 0,043 \\
\hline$x($ Alter $=64)$ & 0,99989 & 0,0 & 1,00112 & 0,084 \\
\hline$x($ Alter $>64)$ & & 0,000 & 1,00546 & 0,000 \\
\hline Kumuliertes, sv-pflichtiges Einkommen [in $€$ ] & 0,99999 & 0,000 & 0,99999 & 0,000 \\
\hline
\end{tabular}


Tab. 9: $\quad$ Cox proportional hazard rate- Schätzungen - Westdeutschland - fortgesetzt -

\begin{tabular}{|c|c|c|c|c|}
\hline \multirow[b]{2}{*}{ Betriebliche Variablen } & \multicolumn{2}{|c|}{ Männer West } & \multicolumn{2}{|c|}{ Frauen West } \\
\hline & $\mathrm{h}$ - ratio & $P>|z|$ & $\mathrm{h}$ - ratio & $P>|z|$ \\
\hline \multicolumn{5}{|l|}{ Institutionelles } \\
\hline Tarifvertrag & \multicolumn{4}{|c|}{ Referenz: kein Tarifvertrag } \\
\hline Branchentarifvertrag (Dummy) & 1,184 & 0,000 & 1,077 & 0,010 \\
\hline Haus-/Firmentarifvertrag (Dummy) & 1,171 & 0,000 & 0,983 & 0,632 \\
\hline Betriebsrat (Dummy) & 1,146 & 0,000 & 1,156 & 0,000 \\
\hline Geltung des KSchG (Dummy) & 1,232 & 0,048 & 1,103 & 0,365 \\
\hline \multicolumn{5}{|l|}{ Anpassungskosten } \\
\hline $\begin{array}{l}\text { Erwartete Entwicklung laufendes Jahr } \\
\text { [in \% Änderung des Geschäftsvolumens] }\end{array}$ & 0,992 & 0,000 & 0,994 & 0,000 \\
\hline Anteil Azubis [in \%] & 1,049 & 0,000 & 1,021 & 0,000 \\
\hline Anteil befristet Angestellter [in \%] & 1,003 & 0,000 & 1,0001 & 0,867 \\
\hline Anteil sonstiger Angestellter [in \%] & 1,019 & 0,387 & 0,997 & 0,921 \\
\hline \multicolumn{5}{|l|}{ Altersstruktur } \\
\hline Anteil Über-50-Jähriger [in \%] & 0,989 & 0,000 & 0,988 & 0,000 \\
\hline $\begin{array}{l}x(\text { Alter }=59) \\
\times(\text { Alter }=60)\end{array}$ & 0,997 & 0,019 & $\begin{array}{l}1,009 \\
1.012\end{array}$ & $\begin{array}{l}0,005 \\
0,000\end{array}$ \\
\hline$x($ Alter $=61)$ & & & 0,995 & 0,093 \\
\hline$x($ Alter $=62)$ & 0,990 & 0,000 & 0,988 & 0,000 \\
\hline$x($ Alter=63) & 10004 & 0832 & 0,992 & 0,029 \\
\hline$x($ Alter $=64)$ & 1,0004 & 0,832 & 0,979 & 0,000 \\
\hline$x($ Alter $>64)$ & 1,008 & 0,000 & 0,996 & 0,194 \\
\hline Anteil Bis-30-Jähriger [in \%] & 0,964 & 0,000 & 0,992 & 0,000 \\
\hline$x($ Alter $=59)$ & 1.016 & 0.000 & 1,002 & 0,474 \\
\hline$x($ Alter $=60)$ & & & 1,0003 & 0,888 \\
\hline $\mathrm{x}($ Alter $=61)$ & & 0000 & 0,987 & 0,000 \\
\hline $\mathrm{x}$ (Alter=62) & 1,011 & 0,000 & 0,980 & 0,000 \\
\hline $\mathrm{x}$ (Alter=63) & & & 0,973 & 0,000 \\
\hline$x($ Alter $=64)$ & 1,016 & 0,000 & 0,982 & 0,000 \\
\hline$x($ Alter $>64)$ & 1,023 & 0,000 & 0,986 & 0,000 \\
\hline \multicolumn{5}{|l|}{ Qualifikationsstruktur } \\
\hline Anteil ungelernter Arbeiter [in \%] & 1,035 & 0,000 & 1,013 & 0,001 \\
\hline Anteil Facharbeiter [in \%] & 1,033 & 0,000 & 1,013 & 0,001 \\
\hline Anteil einfacher Angestellter/Beamter [in \%] & 1,038 & 0,000 & 1,014 & 0,000 \\
\hline Anteil qualifizierter Angestellter/Beamter [in \%] & 1,034 & 0,000 & 1,013 & 0,001 \\
\hline \multicolumn{5}{|l|}{ Technischer Wandel } \\
\hline Technischer Stand der Anlagen & \multicolumn{4}{|c|}{ Referenz: mittel } \\
\hline Neu (Dummy) & 1,026 & 0,293 & 1,258 & 0,000 \\
\hline x (Alter=59) & 0,940 & 0,045 & 0,848 & 0,016 \\
\hline$x($ Alter $=60)$ & 0,940 & 0,045 & 0,803 & 0,000 \\
\hline$x($ Alter $=61)$ & 1,005 & 0,891 & 0,854 & 0,008 \\
\hline$x($ Alter $=62)$ & 1,005 & 0,891 & 0,775 & 0,000 \\
\hline$x($ Alter=63) & 0.895 & 0.001 & 0,725 & 0,000 \\
\hline$x($ Alter $=64)$ & 0,895 & 0,007 & 0,656 & 0,000 \\
\hline$x($ Alter $>64)$ & 1,010 & 0,814 & 0,625 & 0,000 \\
\hline Alt (Dummy) & 1,407 & 0,000 & 1,336 & 0,003 \\
\hline$x($ Alter $=59)$ & ?20? & 0817 & 1,299 & 0,127 \\
\hline$x($ Alter $=60)$ & & & 0,803 & 0,061 \\
\hline$x($ Alter $=61)$ & 0994 & 0955 & 0,952 & 0,757 \\
\hline$x($ Alter $=62)$ & & & 0,877 & 0,492 \\
\hline$x($ Alter $=63)$ & 0847 & 0.104 & 0,850 & 0,461 \\
\hline$x($ Alter $=64)$ & 0,847 & 0,104 & 0,752 & 0,338 \\
\hline$x($ Alter $>64)$ & 0,748 & 0,034 & 0,518 & 0,001 \\
\hline Investitionen in Kommunikationstechnik (Dummy) & 0,983 & 0,292 & 0,970 & 0,150 \\
\hline Anteil der Investitionen am Umsatz [in \%] & 0,99996 & 0,290 & 0,99999 & 0,730 \\
\hline
\end{tabular}


Tab. 9: $\quad$ Cox proportional hazard rate- Schätzungen - Westdeutschland - fortgesetzt -

\begin{tabular}{|c|c|c|c|c|}
\hline \multirow[b]{2}{*}{ Betriebliche Variablen } & \multicolumn{2}{|c|}{ Männer West } & \multicolumn{2}{|c|}{ Frauen West } \\
\hline & h - ratio & $P>|z|$ & h - ratio & $P>|z|$ \\
\hline \multicolumn{5}{|l|}{ Sonstige Kontrollvariablen } \\
\hline Branche & \multicolumn{4}{|c|}{ Referenz: Öffentlicher Sektor } \\
\hline Agrar, Energie, Bergbau, Wasser & 3,406 & 0,000 & 2,706 & 0,000 \\
\hline $\begin{array}{l}\text { Verarbeitendes Gewerbe - Mittel- bis Hoch- } \\
\text { technologie }\end{array}$ & 1,757 & 0,000 & 1,684 & 0,000 \\
\hline Verarbeitendes Gewerbe - Niedrigtechnologie & 1,483 & 0,000 & 1,341 & 0,000 \\
\hline Baugewerbe & 1,875 & 0,000 & 1,401 & 0,000 \\
\hline Handel & 1,218 & 0,000 & 0,984 & 0,693 \\
\hline Verkehr & 1,505 & 0,000 & 1,428 & 0,000 \\
\hline Finanzdienstleistungen & 1,702 & 0,000 & 1,832 & 0,000 \\
\hline Wissensintensive Hochtechnologie- & & & & \\
\hline Dienstleistungen & 1,564 & 0,000 & 1,466 & 0,000 \\
\hline $\begin{array}{l}\text { Wissensintensive Unternehmensdienstleistun- } \\
\text { gen }\end{array}$ & 2,113 & 0,000 & 1,614 & 0,000 \\
\hline Operative Dienstleistungen & 0,691 & 0,000 & 0,780 & 0,002 \\
\hline Gesundheitswesen & 0,803 & 0,000 & 0,922 & 0,004 \\
\hline Sonstige personenbezogene Dienstleistungen & 1,149 & 0,000 & 1,148 & 0,000 \\
\hline Grundstückswesen & 1,772 & 0,000 & 1,430 & 0,000 \\
\hline Rechtsform & \multicolumn{4}{|c|}{ Referenz: Körperschaft öffentlichen Rechts } \\
\hline Einzelunternehmen & 0,663 & 0,000 & 0,788 & 0,003 \\
\hline Personengesellschaft & 0,787 & 0,000 & 0,878 & 0,002 \\
\hline $\mathrm{GmbH}$ & 0,846 & 0,000 & 0,905 & 0,000 \\
\hline Kapitalgesellschaft & 0,958 & 0,074 & 1,064 & 0,041 \\
\hline Sonstige Rechtsform & 0,797 & 0,000 & 0,956 & 0,147 \\
\hline Stellung in Unternehmensorganisation & \multicolumn{4}{|c|}{ Referenz: Niederlassung } \\
\hline Einzelbetrieb & 0,966 & 0,006 & 1,018 & 0,335 \\
\hline Zentrale & 1,175 & 0,000 & 1,206 & 0,000 \\
\hline Mittelinstanz & 0,882 & 0,000 & 1,014 & 0,634 \\
\hline Betriebsgröße & \multicolumn{4}{|c|}{ Referenz: 100 bis 199 Beschäftigte } \\
\hline 1 bis 4 Beschäftigte & 1,831 & 0,004 & 1,650 & 0,019 \\
\hline 5 bis 9 Beschäftigte & 1,431 & 0,002 & 0,825 & 0,201 \\
\hline 10 bis19 Beschäftigte & 1,007 & 0,920 & 0,842 & 0,032 \\
\hline 20 bis 49 Beschäftigte & 0,892 & 0,005 & 0,814 & 0,000 \\
\hline 50 bis 99 Beschäftigte & 0,913 & 0,006 & 0,931 & 0,103 \\
\hline 200 bis 499 Beschäftigte & 1,137 & 0,000 & 1,002 & 0,960 \\
\hline 500 bis 999 Beschäftigte & 1,272 & 0,000 & 1,117 & 0,001 \\
\hline 1000 bis 4999 Beschäftigte & 1,540 & 0,000 & 1,426 & 0,000 \\
\hline 5000 und mehr Beschäftigte & 2,505 & 0,000 & 1,707 & 0,000 \\
\hline Bundesland & \multicolumn{4}{|c|}{ Referenz: Niedersachsen } \\
\hline Berlin - West & 1,620 & 0,000 & 1,419 & 0,000 \\
\hline Schleswig-Holstein & 0,833 & 0,000 & 0,897 & 0,012 \\
\hline Hamburg & 1,113 & 0,000 & 1,021 & 0,540 \\
\hline Bremen & 1.073 & 0.039 & 0.801 & 0.000 \\
\hline Nordrhein-Westfalen & 1,251 & 0,000 & 0,969 & 0,250 \\
\hline Hessen & 0,967 & 0,165 & 0,865 & 0,000 \\
\hline Baden-Württemberg & 1,095 & 0,000 & 0,979 & 0,461 \\
\hline Bayern & 1,160 & 0,000 & 0,937 & 0,026 \\
\hline Rheinland-Pfalz und Saarland & 0,817 & 0,000 & 0,721 & 0,000 \\
\hline
\end{tabular}


Tab. 9: $\quad$ Cox proportional hazard rate- Schätzungen - Westdeutschland - fortgesetzt -

\begin{tabular}{lcccc}
\hline & \multicolumn{2}{c}{ Männer West } & \multicolumn{2}{c}{ Frauen West } \\
Rentenzugangsrecht & $\mathbf{h}$ - ratio & $\mathbf{P > | \mathbf { z } |}$ & $\mathbf{h}-$ ratio & $\mathbf{P}>|\mathbf{z}|$ \\
\hline \hline Altersrente nach Arbeitslosigkeit oder Altersteilzeit & & & & \\
$\quad$ Ohne Abschlag & 0,491 & 0,000 & 1,586 & 0,000 \\
$\quad$ Mit Abschlag & 0,575 & 0,000 & 1,385 & 0,000 \\
$\quad$ Max Abschlag & 0,416 & 0,000 & 0,967 & 0,625 \\
Altersrente für Frauen & & & & \\
$\quad$ Ohne Abschlag & - & - & - & - \\
$\quad$ Mit Abschlag & - & - & 0,604 & 0,000 \\
$\quad$ Max Abschlag & - & - & - & - \\
Altersrente für Schwerbehinderte & & & & \\
$\quad$ Ohne Abschlag & 0,872 & 0,000 & 3,226 & 0,000 \\
$\quad$ Mit Abschlag & - & - & - & - \\
$\quad$ Max Abschlag & - & - & - & - \\
Altersrente für langjährig Versicherte & & & & \\
$\quad$ Ohne Abschlag & 2,838 & 0,000 & 1,914 & 0,000 \\
$\quad$ Mit Abschlag & 2,150 & 0,000 & 1,519 & 0,000 \\
$\quad$ Max Abschlag & 1,038 & 0,448 & 1,083 & 0,369 \\
\hline Erwerbsminderungsrente & & & & \\
$\quad$ Ohne Abschlag & 11,918 & 0,000 & 3,369 & 0,000 \\
$\quad$ Mit Abschlag & 11,119 & 0,000 & 5,058 & 0,000 \\
\hline
\end{tabular}

Quelle: LIAB; eigene Berechnungen

Die folgende Tab. gibt in der linken Hälfte die Schätzungen für ostdeutsche Männer wieder. Sie beruhen auf den Daten von 80425 Männern, von denen ein gutes Viertel am Ende des Beobachtungszeitraumes noch sozialversicherungspflichtiger Beschäftigung nachgeht. Der zusammenfassende $\mathrm{Chi}^{2}$-Test mit einem empirischen Signifikanzniveau von nahe Null attestiert dem gewählten Variablenkanon wiederum gemeinsam hochsignifikanten Einfluss auf das zu erklärende Austrittsverhalten. Daneben sind die Regressionsergebnisse der CoxSchätzungen für die ostdeutschen Frauen dargestellt. Sie stützen sich auf 66026 Personen. Es fällt auf, dass für diese Gruppe der Anteil der am Ende des Jahres 2003 in Beschäftigung befindlichen Personen mit einem Drittel deutlich über dem Verbleibeanteil der anderen Gruppen liegt. Wieder weist der $\mathrm{Chi}^{2}$-Test eine hohe gemeinsame Signifikanz der Regressoren aus. 
Tab. 10: Cox proportional hazard rate- Schätzungen - Ostdeutschland

\begin{tabular}{|c|c|c|c|c|}
\hline Männer Ost & \multicolumn{4}{|c|}{ Frauen Ost } \\
\hline $\begin{array}{l}\text { No. of subjects }= \\
\text { No. of failures }= \\
\text { Time at risk = } \\
\text { Log likelihood }= \\
\text { Number of obs }= \\
\text { LR chi2 }(136)= \\
\text { Prob }>\text { chi2 }=\end{array}$ & $\begin{array}{l}\text { No. of subje } \\
\text { No. of failur } \\
\text { Time at risk } \\
\text { Log likeliho } \\
\text { Number of } \\
\text { LR chi2 } 2(13 \\
\text { Prob }>\text { chi2 }\end{array}$ & & & $\begin{array}{r}66026 \\
21643 \\
352335914 \\
-209396,54 \\
156519 \\
28098,01 \\
0,0000\end{array}$ \\
\hline \multirow[b]{2}{*}{ Personenvariablen } & \multicolumn{2}{|c|}{ Männer Ost } & \multicolumn{2}{|c|}{$\begin{array}{ll}\text { Frauen Ost } \\
\end{array}$} \\
\hline & h - ratio & $P>|z|$ & h - ratio & $P>|z|$ \\
\hline Alter (in Jahren) & 1,058 & 0,000 & $1,1,206$ & 0,000 \\
\hline Freistellung in den vergangenen zwei Jahren & 2,713 & 0,000 & 2,777 & 0,000 \\
\hline$x($ Alter=59) & 0,555 & 0,000 & 0,843 & 0,284 \\
\hline$x($ Alter $=60)$ & 0,876 & 0,328 & 0,389 & 0,000 \\
\hline$x($ Alter $=61)$ & 0,817 & 0,276 & 0,496 & 0,032 \\
\hline$x($ Alter $=62)$ & 0,608 & 0,066 & 0,305 & 0,002 \\
\hline$x($ Alter $=63)$ & 0,481 & 0,001 & 0,432 & 0,405 \\
\hline$x($ Alter $=64)$ & 1,395 & 0,315 & 0,698 & 0,617 \\
\hline$x($ Alter>64) & 0,255 & 0,000 & 0,370 & 0,053 \\
\hline Anzahl Freistellungen & 0,903 & 0,326 & 0,798 & 0,090 \\
\hline Anzahl Freistellungen² & 1,078 & 0,255 & 1,110 & 0,241 \\
\hline Eintritt ins Erwerbsleben & 1,00036 & 0,000 & 1.00062 & 0,000 \\
\hline Eintritt ins Erwerbsleben² & 0,99978 & 0,000 & 0,99967 & 0,000 \\
\hline Eintritt in den Betrieb & 1,00348 & 0,000 & 1,00385 & 0,000 \\
\hline Eintritt in den Betrieb² & 0,99861 & 0,000 & 0,99849 & 0,000 \\
\hline Deutsch & 0,470 & 0,000 & 0,263 & 0,000 \\
\hline x (Alter=59) & 2,910 & 0,000 & 1,416 & 0,000 \\
\hline$x($ Alter $=60)$ & 1,250 & 0,089 & 2,547 & 0,000 \\
\hline$x($ Alter $=61)$ & 0,906 & 0,492 & 0,807 & 0,137 \\
\hline x (Alter=62) & 0,953 & 0,756 & 0,483 & 0,000 \\
\hline$x($ Alter $=63)$ & 1,102 & 0,497 & 0,481 & 0,004 \\
\hline$x($ Alter $=64)$ & 0,858 & 0,404 & 0,333 & 0,000 \\
\hline$x($ Alter $>64)$ & 0,992 & 0,946 & 0,609 & 0,005 \\
\hline Berufliche Stellung & \multicolumn{4}{|c|}{ Referenzkategorie: Angestellte/r } \\
\hline Einfacher Arbeiter & 1,071 & 0,014 & 0,960 & 0,158 \\
\hline Facharbeiter & 0,998 & 0,924 & 0,848 & 0,000 \\
\hline Meister & 1,099 & 0,038 & 1,037 & 0,830 \\
\hline Teilzeit $<15$ Std./Woche & 0,385 & 0,000 & 0,282 & 0,000 \\
\hline Teilzeit >15 Std./Woche & 1,229 & 0,000 & 0,855 & 0,000 \\
\hline Schul-/Ausbildung & \multicolumn{4}{|c|}{ Referenzkategorie: Hochschulabschluss } \\
\hline Bis mittlere Reife ohne Berufsausbildung & 1,225 & 0,000 & 1,023 & 0,606 \\
\hline Bis mittlere Reife mit Berufsausbildung & 1,165 & 0,000 & 1,090 & 0,019 \\
\hline (Fach-) Hochschulreife ohne Berufsausbildung & 1,369 & 0,014 & 0,937 & 0,692 \\
\hline (Fach-) Hochschulreife mit Berufsausbildung & 1,186 & 0,000 & 1,039 & 0,456 \\
\hline Fachhochschulabschluss & 1,322 & 0,000 & 1,246 & 0,000 \\
\hline Tagesentgelt [in $€$ ] & 1,007 & 0,000 & 1,008 & 0,000 \\
\hline$x($ Alter $=59)$ & 0,999 & 0,019 & 0,999 & 0,245 \\
\hline$x($ Alter $=60)$ & 0,999 & 0,003 & 0.999 & 0,055 \\
\hline$x($ Alter $=61)$ & 0,997 & 0,000 & 1,003 & 0,000 \\
\hline$x($ Alter $=62)$ & 0,999 & 0,307 & 1,011 & 0,000 \\
\hline$x($ Alter $=63)$ & 1,003 & 0,000 & 1,009 & 0,000 \\
\hline$x($ Alter $=64)$ & 1,002 & 0,001 & 1,006 & 0,000 \\
\hline$x($ Alter $>64)$ & 1,010 & 0,000 & 1,014 & 0,000 \\
\hline $\begin{array}{l}\text { Kumuliertes, sozialversicherungspflichtiges Ein- } \\
\text { kommen [in } € \text { ] }\end{array}$ & 0,99999 & 0,000 & 0,99998 & 0,000 \\
\hline
\end{tabular}


Tab. 10: $\quad$ Cox proportional hazard rate- Schätzungen - Ostdeutschland - fortgesetzt -

\begin{tabular}{|c|c|c|c|c|}
\hline \multirow[b]{2}{*}{ Betriebliche Variablen } & \multicolumn{2}{|c|}{ Männer Ost } & \multicolumn{2}{|c|}{ Frauen Ost } \\
\hline & h - ratio & $P>|z|$ & h - ratio & $P>|z|$ \\
\hline \multicolumn{5}{|l|}{ Institutionelles } \\
\hline Tarifvertrag & \multicolumn{4}{|c|}{ Referenz: kein Tarifvertrag } \\
\hline Branchentarifvertrag (Dummy) & 1,157 & 0,000 & 1,139 & 0,000 \\
\hline Haus-/Firmentarifvertrag (Dummy) & 1,049 & 0,038 & 1,061 & 0,026 \\
\hline Betriebsrat (Dummy) & 1,250 & 0,000 & 1,155 & 0,000 \\
\hline Geltung des KSchG (Dummy) & 0,960 & 0,719 & 1,128 & 0,315 \\
\hline \multicolumn{5}{|l|}{ Anpassungskosten } \\
\hline $\begin{array}{l}\text { Erwartete Entwicklung laufendes Jahr } \\
\text { [in \% Änderung des Geschäftsvolumens] }\end{array}$ & 0,990 & 0,000 & 0,991 & 0,000 \\
\hline Anteil Azubis [in \%] & 1,017 & 0,000 & 1,021 & 0,000 \\
\hline Anteil befristet Angestellter [in \%] & 1,007 & 0,000 & 1,006 & 0,000 \\
\hline Anteil sonstiger Angestellter [in \%] & 1,008 & 0,500 & 0,974 & 0,174 \\
\hline \multicolumn{5}{|l|}{ Altersstruktur } \\
\hline Anteil Über-50-Jähriger [in \%] & 1,002 & 0,165 & 0,991 & 0,000 \\
\hline$x($ Alter $=59)$ & 0,990 & 0,000 & 1,002 & 0,372 \\
\hline$x($ Alter $=60)$ & 0,984 & 0,000 & 0,987 & 0,000 \\
\hline$x($ Alter $=61)$ & 0,990 & 0,000 & 0,993 & 0,008 \\
\hline$x($ Alter=62) & 0,986 & 0,000 & 0,988 & 0,000 \\
\hline$x($ Alter $=63)$ & 0,983 & 0,000 & 0,970 & 0,000 \\
\hline$x($ Alter $=64)$ & 0,984 & 0,000 & 0,985 & 0,048 \\
\hline$x($ Alter $>64)$ & 0,982 & 0,000 & 0,969 & 0,000 \\
\hline Anteil Bis-30-Jähriger [in \%] & 0,995 & 0,000 & 0,987 & 0,000 \\
\hline$x($ Alter $=59)$ & 1,004 & 0,031 & 1,005 & 0,029 \\
\hline$x($ Alter $=60)$ & 1,002 & 0,350 & 0,9995 & 0,790 \\
\hline$x($ Alter $=61)$ & 1,006 & 0,004 & 0,995 & 0,081 \\
\hline$x($ Alter $=62)$ & 1,001 & 0,627 & 0,993 & 0,054 \\
\hline$x($ Alter $=63)$ & 0,991 & 0,000 & 0,989 & 0,048 \\
\hline$x($ Alter $=64)$ & 0,987 & 0,001 & 0,995 & 0,480 \\
\hline$x($ Alter $>64)$ & 0,984 & 0,000 & 0,974 & 0,000 \\
\hline \multicolumn{5}{|l|}{ Qualifikationsstruktur } \\
\hline Anteil ungelernter Arbeiter [in \%] & 1,010 & 0,005 & 1,011 & 0,983 \\
\hline Anteil Facharbeiter [in \%] & 1,012 & 0,000 & 1,012 & 0,983 \\
\hline Anteil einfacher Angestellter/Beamter [in \%] & 1,012 & 0,001 & 1,011 & 0,983 \\
\hline Anteil qualifizierter Angestellter/Beamter [in \%] & 1,012 & 0,000 & 1,012 & 0,983 \\
\hline \multicolumn{5}{|l|}{ Technischer Wandel } \\
\hline Technischer Stand der Anlagen & \multicolumn{4}{|c|}{ Referenz: mittel } \\
\hline Neu (Dummy) & 0,983 & 0,528 & 0,983 & 0,478 \\
\hline x (Alter=59) & 0,815 & 0,000 & 0,837 & 0,000 \\
\hline$x($ Alter $=60)$ & 1,060 & 0,197 & 1,128 & 0,001 \\
\hline$x($ Alter $=61)$ & 1,011 & 0,842 & 1,032 & 0,596 \\
\hline$x($ Alter $=62)$ & 1,136 & 0,044 & 0,950 & 0,461 \\
\hline$x($ Alter $=63)$ & 0,927 & 0,168 & 1,040 & 0,753 \\
\hline$x($ Alter $=64)$ & 0,973 & 0,752 & 1,163 & 0,330 \\
\hline x (Alter>64) & 0,900 & 0,050 & 0,735 & 0,001 \\
\hline Alt (Dummy) & 0,847 & 0,001 & 0,910 & 0,033 \\
\hline$x($ Alter=59) & 1,180 & 0,040 & 1,138 & 0,095 \\
\hline$x($ Alter $=60)$ & 1,287 & 0,007 & 0,981 & 0,808 \\
\hline$x($ Alter $=61)$ & 1,262 & 0,054 & 1,060 & 0,697 \\
\hline$x($ Alter=62) & 1,500 & 0,005 & 0,984 & 0,930 \\
\hline$x($ Alter $=63)$ & 1,009 & 0,950 & 0,851 & 0,598 \\
\hline$x($ Alter $=64)$ & 1,345 & 0,164 & 1,010 & 0,981 \\
\hline$x($ Alter $>64)$ & 0,973 & 0,834 & 0,733 & 0,214 \\
\hline Investitionen in Kommunikationstechnik (Dummy) & 0,914 & 0,000 & 1,006 & 0,754 \\
\hline Anteil der Investitionen am Umsatz [in \%] & 1,0002 & 0,186 & 1,001 & 0,000 \\
\hline
\end{tabular}


Tab. 10: $\quad$ Cox proportional hazard rate- Schätzungen - Ostdeutschland - fortgesetzt -

\begin{tabular}{|c|c|c|c|c|}
\hline \multirow[b]{2}{*}{ Betriebliche Variablen } & \multicolumn{2}{|c|}{ Männer Ost } & \multicolumn{2}{|c|}{ Frauen Ost } \\
\hline & h - ratio & $P>|z|$ & h - ratio & $\mathbf{P}>|\mathbf{z}|$ \\
\hline \multicolumn{5}{|l|}{ Sonstige Kontrollvariablen } \\
\hline Branche & \multicolumn{4}{|c|}{ Referenz: Öffentlicher Sektor } \\
\hline Agrar, Energie, Bergbau, Wasser & 1,038 & 0,202 & 1,165 & 0,000 \\
\hline $\begin{array}{l}\text { Verarbeitendes Gewerbe - Mittel- bis Hoch- } \\
\text { technologie }\end{array}$ & 0,602 & 0,000 & 0,793 & 0,000 \\
\hline Verarbeitendes Gewerbe - Niedrigtechnologie & 0,676 & 0,000 & 0,758 & 0,000 \\
\hline Baugewerbe & 1,113 & 0,001 & 0,983 & 0,725 \\
\hline Handel & 0,689 & 0,000 & 0,724 & 0,000 \\
\hline Verkehr & 0,668 & 0,000 & 0,801 & 0,001 \\
\hline Finanzdienstleistungen & 0,856 & 0,166 & 1,214 & 0,000 \\
\hline Wissensintensive Hochtechnologie- & & & & \\
\hline Dienstleistungen & 0,646 & 0,000 & 0,835 & 0,001 \\
\hline $\begin{array}{l}\text { Wissensintensive Unternehmensdienstleistun- } \\
\text { gen }\end{array}$ & 1,007 & 0,912 & 1,174 & 0,018 \\
\hline Operative Dienstleistungen & 0,525 & 0,000 & 0,552 & 0,000 \\
\hline Gesundheitswesen & 0,583 & 0,000 & 0,810 & 0,000 \\
\hline Sonstige personenbezogene Dienstleistungen & 0,828 & 0,000 & 0,807 & 0,000 \\
\hline Grundstückswesen & 0,971 & 0,649 & 1,883 & 0,000 \\
\hline Rechtsform & \multicolumn{4}{|c|}{ Referenz: Körperschaft öffentlichen Rechts } \\
\hline Einzelunternehmen & 1,296 & 0,001 & 0,638 & 0,000 \\
\hline Personengesellschaft & 1,496 & 0,000 & 1,083 & 0,228 \\
\hline $\mathrm{GmbH}$ & 1,549 & 0,000 & 1,108 & 0,000 \\
\hline Kapitalgesellschaft & 2,340 & 0,000 & 1,816 & 0,000 \\
\hline Sonstige Rechtsform & 1,470 & 0,000 & 1,239 & 0,000 \\
\hline Stellung in Unternehmensorganisation & \multicolumn{4}{|c|}{ Referenz: Niederlassung } \\
\hline Einzelbetrieb & 1,120 & 0,000 & 1,050 & 0,039 \\
\hline Zentrale & 1,098 & 0,002 & 1,101 & 0,001 \\
\hline Mittelinstanz & 1,047 & 0,167 & 1,132 & 0,000 \\
\hline Betriebsgröße & \multicolumn{4}{|c|}{ Referenz: 100 bis 199 Beschäftigte } \\
\hline 1 bis 4 Beschäftigte & 1,284 & 0,166 & 1,856 & 0,000 \\
\hline 5 bis 9 Beschäftigte & 0,972 & 0,802 & 0,983 & 0,895 \\
\hline 10 bis19 Beschäftigte & 0,969 & 0,612 & 1,126 & 0,075 \\
\hline 20 bis 49 Beschäftigte & 0,934 & 0,065 & 0,935 & 0,114 \\
\hline 50 bis 99 Beschäftigte & 0,858 & 0,000 & 0,908 & 0,009 \\
\hline 200 bis 499 Beschäftigte & 0,984 & 0,489 & 1,004 & 0,886 \\
\hline 500 bis 999 Beschäftigte & 1,038 & 0,120 & 1,054 & 0,035 \\
\hline 1000 bis 4999 Beschäftigte & 1,157 & 0,000 & 1,168 & 0,000 \\
\hline 5000 und mehr Beschäftigte & 0,983 & 0,810 & 0,890 & 0,067 \\
\hline Bundesland & \multicolumn{4}{|c|}{ Referenz: Westl. Bundesländer } \\
\hline Berlin - Ost & 0,631 & 0,001 & 1,272 & 0,361 \\
\hline Brandenburg & 0,590 & 0,000 & 1,261 & 0,377 \\
\hline Mecklenburg-Vorpommern & 0,550 & 0,000 & 1,193 & 0,501 \\
\hline Sachsen & 0,633 & 0,001 & 1,295 & 0,324 \\
\hline Sachsen-Anhalt & 0,628 & 0,000 & 1,201 & 0,484 \\
\hline Thüringen & 0,584 & 0,000 & 1,149 & 0,596 \\
\hline
\end{tabular}


Tab. 10: $\quad$ Cox proportional hazard rate- Schätzungen - Ostdeutschland - fortgesetzt -

\begin{tabular}{lcccc}
\hline & \multicolumn{2}{c}{ Männer Ost } & \multicolumn{2}{c}{ Frauen Ost } \\
Rentenzugangsrecht & $\mathbf{h}$ - ratio & $\mathbf{P > | \mathbf { z } |}$ & $\mathbf{h}$ - ratio & $\mathbf{P}>\mathbf{z} \mid$ \\
\hline \hline Altersrente nach Arbeitslosigkeit oder Altersteilzeit & & & & \\
$\quad$ Ohne Abschlag & 0,687 & 0,000 & 0,552 & 0,000 \\
$\quad$ Mit Abschlag & 0,963 & 0,601 & 1,440 & 0,000 \\
$\quad$ Max Abschlag & 0,918 & 0,365 & 1,040 & 0,634 \\
Altersrente für Frauen & & & & \\
$\quad$ Ohne Abschlag & - & - & - & - \\
$\quad$ Mit Abschlag & - & - & 0,901 & 0,004 \\
$\quad$ Max Abschlag & - & - & - & - \\
Altersrente für Schwerbehinderte & & & & \\
$\quad$ Ohne Abschlag & 4,474 & 0,000 & 2,763 & 0,000 \\
$\quad$ Mit Abschlag & 3,351 & 0,000 & 0,600 & 0,001 \\
$\quad$ Max Abschlag & - & - & - & - \\
Altersrente für langjährig Versicherte & & & & \\
$\quad$ Ohne Abschlag & 2,272 & 0,000 & 1,515 & 0,000 \\
$\quad$ Mit Abschlag & 2,623 & 0,000 & 3,827 & 0,000 \\
$\quad$ Max Abschlag & 1,587 & 0,000 & 1,758 & 0,000 \\
\hline Erwerbsminderungsrente & & & & \\
$\quad$ Ohne Abschlag & - & - & - & - \\
$\quad$ Mit Abschlag & 0,814 & 0,000 & 2,009 & 0,000 \\
\hline
\end{tabular}

Quelle: LIAB, eigene Berechnungen

Nach der reinen Darstellung sollen im Folgenden die Ergebnisse der persönlichen Variablen zusammenfassend für alle Einzelschätzungen beschrieben, interpretiert und mit dem bisherigen Forschungsstand verglichen werden.

Vor der nun folgenden Interpretation und Diskussion der Ergebnisse muss noch einmal auf den selektiven Charakter des untersuchten Samples hingewiesen werden. Die Ergebnisse lassen sich - sofern sie denn signifikant sind - nur auf die beschriebene Gesamtheit übertragen. Gemein ist den Personen, dass sie alle mindestens eine abhängige Beschäftigungsbeobachtung nach dem 50. Lebensjahr aufweisen. Besondere Problemfälle des Arbeitsmarktes wie Langzeitarbeitslose oder Personen, denen die zum Untersuchungszeitpunkt noch durchlässigeren Zugangswege zur Erwerbs- oder Berufsunfähigkeit offen standen, sind von der Analyse und damit der folgenden Interpretation ausgenommen. Dasselbe gilt für ausschließlich Selbstständige. Da in die Schätzung nur solche Perioden von Personen eingehen, in denen sie für einen Panelbetrieb gearbeitet haben, ist die Stichprobe auch dahingehend verzerrt, als Personen mit geringer Wechselneigung und stabilen Beschäftigungsverhältnissen überrepräsentiert sind. 


\section{A 5.1 Einfluss von Personenvariablen}

Die Kontrollvariable Alter erweist sich in jeder der Schätzungen als hoch signifikanter Einflussfaktor. Sie wirkt sich in Westdeutschland negativ, in Ostdeutschland positiv auf die Hazardrate aus. Mit zunehmendem Alter sinkt also in den alten Bundesländern die bedingte Übergangswahrscheinlichkeit. Dieses erste zunächst überraschende Teilergebnis muss allerdings vor dem Hintergrund betrachtet werden, dass das Alter - gemessen in Jahren - nicht nur an dieser Stelle in die Schätzungen eingeht. Vielmehr taucht es an mehreren Stellen mit anderen Variablen „gekreuzt“ auf, um den lebenszeitlichen Verlauf des Einflusses dieser Merkmale näher zu untersuchen und die Annahmen des proportionalen Hazardratenmodells aufzuweichen. Dennoch bleibt der Unterschied zwischen den beiden Landesteilen festzuhalten. In Ostdeutschland fallen demnach die Beschäftigungschancen mit zunehmendem Alter stärker ab, als dies für den Westen gilt. Zu beachten ist, dass sich diese Aussage auf eine selektive Stichprobe der untersuchten Jahrgänge 1945 und älter und damit auf eine Generation bezieht, die mindestens die erste Hälfte ihres Erwerbslebens in der DDR verbracht haben und somit eine rasche Abschreibung ihres Humankapitals hinnehmen mussten. Der gemessene Unterschied mag sich daher auch durch den besonders starken Wandel der Arbeitsanforderungen nach der Wende und die dadurch beschleunigte Abschreibung erworbenen Humankapitals erklären.

Einheitlicher gestalten sich die Schätzergebnisse für die Variablen, die den Einfluss des Gesundheitszustandes ausdrücken sollen. Um den aktuellen gesundheitlichen Zustand zu kontrollieren, wurde eine Variable gebildet, die angibt, ob in den jeweils zurückliegenden zwei Jahren ein Zeitraum sozialversicherungspflichtiger Beschäftigung beobachtet wurde, in dem die Person kein Entgelt erhielt („Freistellungsspell“). Diese Dummy-Variable ging auch interagiert mit Altersangaben ein. Um auch längerfristige Auswirkungen von gesundheitlichen Einschränkungen zu berücksichtigen, wurde eine Zählvariable über alle Freistellungsspells, sowie ihr Quadrat als Regressor gewählt.

Was die kurzfristigen Effekte gesundheitlicher Probleme angeht, sind die Ergebnisse in allen Fällen hochsignifikant und die Hazard ratios deutlich vom Wert „1“ verschieden. Dabei zeichnet sich ab, dass die Beobachtung eines Freistellungsspells besonders für die jüngeren unter den Beobachteten eine massive Erhöhung des Austrittsrisikos darstellt. Abb. 14 stellt die Entwicklung des Einflusses über das Lebensalter hinweg dar. Dabei werden neben den geschätzten Koeffizienten auch die Produkte des ursprünglichen Dummies und des jeweiligen Altersdummies angegeben, die den erwarteten, altersspezifischen Einfluss von Erkrankungen darstellen. 
Abb. 14: Hazard ratio der Variablen „Aktueller Freistellungsspell“ im Altersprofil

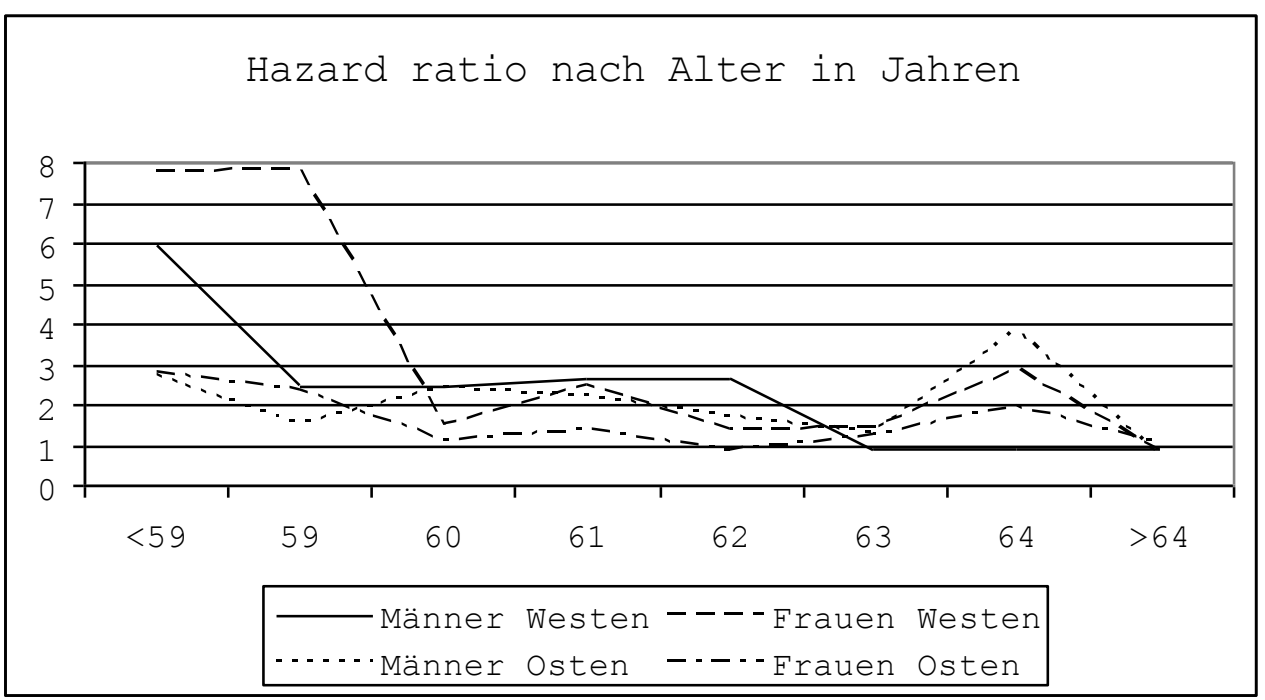

Quelle: LIAB, eigene Berechnungen

Vor dem Erreichen eines Alters von 59 ist das Auftreten einer Erkrankung ein deutliches Anzeichen für einen möglichen baldigen Austritt. In den Altbundesländern erhöht es die Hazardrate um den Faktor sechs bis acht. Wer eine solche Freistellungsphase zu verzeichnen hat, zieht sich in der Folgezeit c.p. mit einer sechs- (Männer) bis knapp achtmal (Frauen) höheren Wahrscheinlichkeit aus dem Erwerbsleben zurück. Für Ostdeutschland sind die Werte mit einer Hazard ratio von bis zu $277 \%$ zwar weit geringer, allerdings immer noch sehr hoch. Möglicherweise kann dieser Unterschied damit erklärt werden, dass die Erwerbsbedingungen in den neuen Ländern unabhängig vom Gesundheitszustand der Person schlechter sind und dieser sich als weniger ausschlaggebend für die untersuchte Fragestellung herausstellt.

Allen Schätzungen ist gemein, dass mit zunehmendem Alter der Einfluss dieser Variable tendenziell schwindet. Für die Ältesten kommt die Schätzung sogar zu einem verlängernden Effekt einer solchen Beobachtung. Trotzdem gilt auch für die meisten Altersgruppen, die vor dem Übergang in die Altersrente stehen, dass ihr Verbleib in Beschäftigung deutlich unwahrscheinlicher wird, sobald eine Periode der Krankheit beobachtet wurde. Die geringer werdenden Effekte lassen sich damit begründen, dass die Hazardraten dieser Altersgruppen ohnehin schon angestiegen sind und eine Verschlechterung der Gesundheit daher nicht mehr einen solch deutlichen Ausschlag auf die Übergangswahrscheinlichkeit haben kann.

Daneben gingen auch die langfristigen Auswirkungen des Gesundheitszustandes als kumulierte bzw. quadrierte, kumulierte Anzahl von Freistellungsspells in die Schätzung ein. Die beiden Variablen bilden die gesundheitliche Historik einer Person ab und erweisen sich als hoch signifikant. Die Schätzergebnisse brachten zunächst unerwartete Ergebnisse. Sie sind in Abb. 15 wegen der im Modell angenommenen Nichtlinearität verdeutlicht: Für Westdeutschland steigt erst ab einer Anzahl von mindestens 13 die Hazard ratio über den Wert „1“ und wirkt erst dann austrittsbeschleunigend. Für die weiblichen, westdeutschen Beschäftigten liegt die Hazard ratio für sinnvolle Einsetzungen der Anzahl unter dem neutralen Wert von eins. 
Lediglich für Ostdeutsche mit mindestens zwei (Männer) bzw. drei (Frauen) Beobachtungen einer Entgeltunterbrechung (trotz Beschäftigung) prognostizieren die Schätzungen einen gegenüber der Referenz keine - erhöhten Wert der Hazardrate. Unterschiede zwischen den beiden Landesteilen mögen auch aus der Tatsache abzuleiten sein, dass die Historik in Ostdeutschland weniger weit zurück reicht.

Abb. 15: Hazard ratio der Variablen „Anzahl der Freistellungsspells“ nach Ausprägung

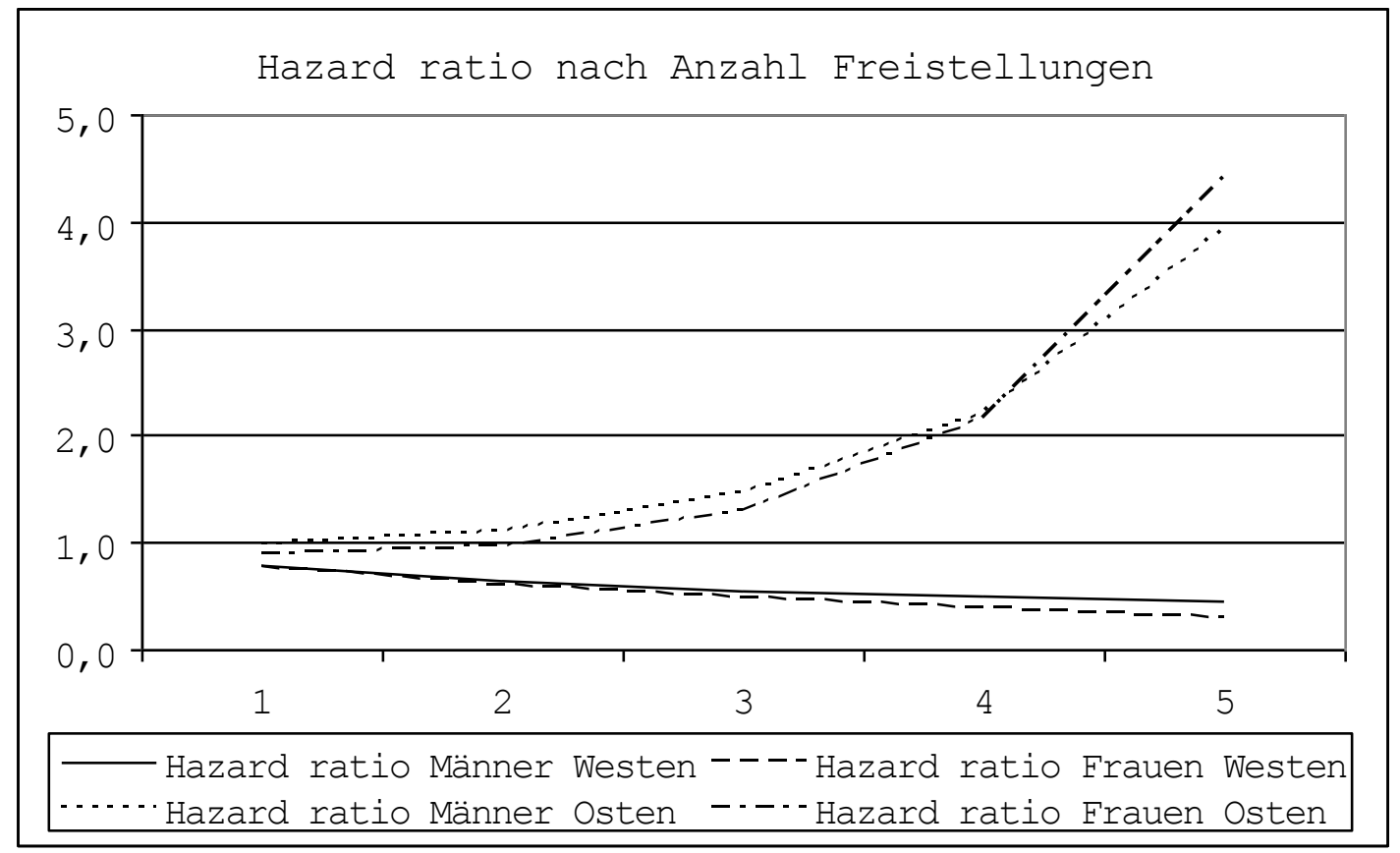

Quelle: LIAB, eigene Berechnungen

Diese Beobachtungen lassen sich verstehen, wenn man beachtet, dass der Umstand einer aktuellen Erkrankung kontrolliert wird und dieser einen sehr deutlichen und auf den Austritt drängenden Effekt ausübt. Die Tatsache, dass Krankheiten überstanden und die Erwerbstätigkeit im Anschluss wieder aufgenommen wurde, kann vor dem Hintergrund dieser Ergebnisse auch als Indiz dafür herangezogen werden, dass die betreffende Person eine besonders starke Stellung innerhalb des Betriebes oder auf dem Arbeitsmarkt generell einnimmt und daher mit c.p. geringerer Wahrscheinlichkeit ausscheidet.

Festzuhalten bleibt, dass die Proxy-Variable einen erheblichen Einfluss auf die Beschäftigungsdynamik Älterer aufweist. Mit diesen Teilergebnissen bestätigt sich, was in vielen Studien belegt wurde: Gesundheit spielt eine wesentliche Rolle bei der Entscheidung, im Alter die Erwerbstätigkeit fortzuführen. Dies deckt sich mit den Befunden aus der deskriptiven Analyse, aber auch von Vorgängerarbeiten wie der von Wübbeke (2005b, S. 88), die ebenfalls zu dem Ergebnis kommt, dass Episoden längerer Krankschreibung eine deutlich erhöhte Austrittswahrscheinlichkeit nach sich ziehen.

Zudem wurde für die Eintrittsdaten in den Betrieb bzw. ins Erwerbsleben kontrolliert. Sie können den Einfluss von Humankapital mit abdecken. Die Einbeziehung beider Variabler lässt eine Unterscheidung zwischen Erfahrung im aktuellen Betrieb und im Arbeitsleben all- 
gemeinem zu. Beide Angaben gehen unverändert und quadriert in die Schätzung ein. Dieses Vorgehen erweist sich als sinnvoll, da beide zugehörigen Parameter von einem hoch signifikanten Einfluss ihrer Variablen zeugen. $\mathrm{Zu}$ beachten ist, dass auch bei diesen Variablen die Historik in Ostdeutschland nur bis zum Jahr 1990 reicht, während für Westdeutschland Eintritte bis in das Jahr 1975 zurückverfolgt werden können.

Betrachtet man zunächst den Eintritt in das Erwerbsleben, so ergibt sich für die geschätzten Parameter ein unerwartetes Ergebnis: Die beiden westdeutschen Schätzungen legen einen c.p. positiven Zusammenhang zwischen der jeweils aktuellen Anzahl der Jahre im Erwerbsleben und der Hazardrate nahe legen. Dies kann möglicherweise damit begründet werden, dass neben der bisherigen Dauer des Erwerbslebens auch für die Zugehörigkeit zum Betrieb kontrolliert wird und eine längere allgemeine Arbeitserfahrung auch für eine - hier nicht beobachtete und nicht kontrollierte - höhere Wechselneigung der Person und damit womöglich auch aus seiner letzten Beschäftigung spricht. In den ostdeutschen Schätzungen wirkt eine längere allgemeine Erfahrung dagegen wie erwartet und senkt die Hazardrate.

Abb. 16: Hazard ratio der Variablen Dauer seit Erwerbseintritt nach Ausprägungen

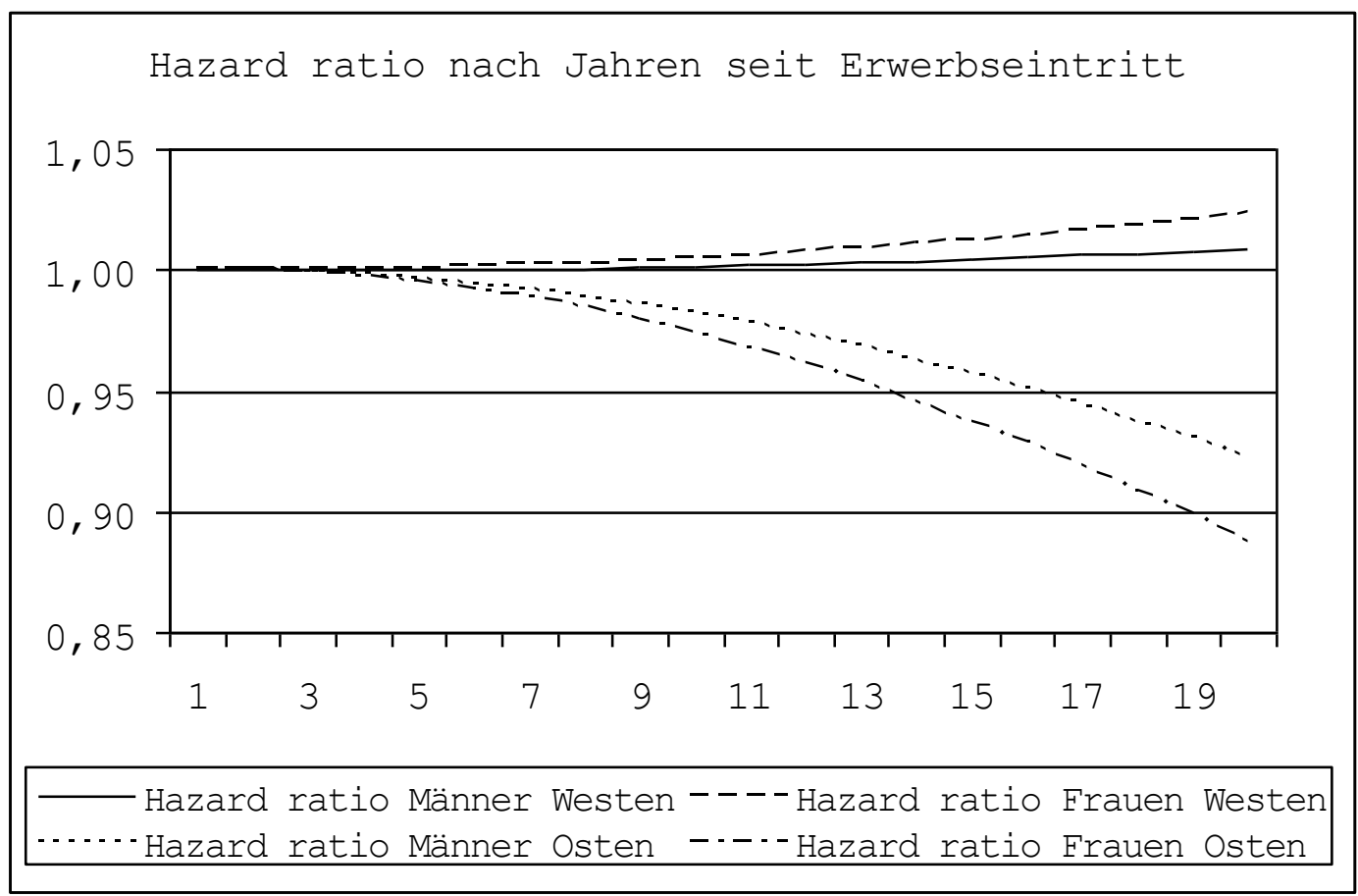

Quelle: LIAB, eigene Berechnungen

Abb. 16 beschreibt die Wirkungen der Betriebszugehörigkeitsdauer. Die Hazard ratios weisen die erwarteten Vorzeichen auf. So gilt für jede der untersuchten Gruppen, dass mit zunehmender Dauer der Zugehörigkeit zum Betrieb die Hazardrate und damit die geschätzte Wahrscheinlichkeit sinkt, in einem gegebenen Zeitraum aus dem Erwerbsleben auszuscheiden. Danach schützt betriebsspezifisches Humankapital wirksam gegen ein vorzeitiges Ausscheiden aus dem Erwerbsleben. Eine banalere Erklärung für den gefundenen Zusammenhang bietet die in A 1.2.4 beschriebene Regelung des BGB zu den vorgeschriebenen Kündigungsfristen, 
die in Abhängigkeit der Betriebszugehörigkeit länger werden. Besonders auffällig ist die deutliche Verringerung der Hazardrate in Ostdeutschland. Hier weisen Personen, die zehn Jahre im selben Betrieb beschäftigt waren, eine um rd. zehn Prozent geringere Übergangsrate auf.

Abb. 17: Hazard ratio der Variablen „Dauer der Betriebszugehörigkeit“ in Jahren

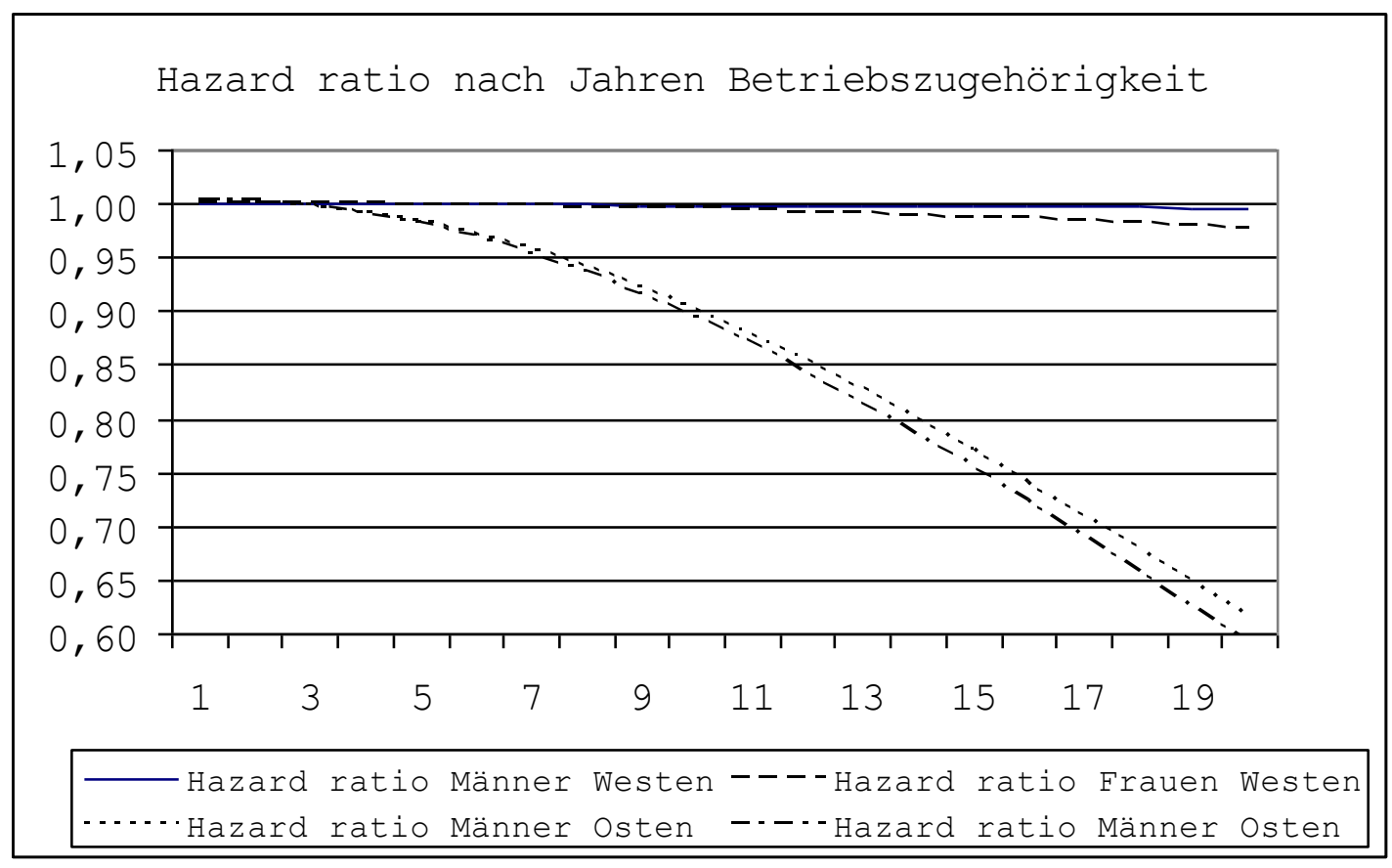

Quelle: LIAB, eigene Berechnungen

Ebenso sprechen Theorien der Stabilität des individuellen Erwerbsverlaufes für eine höhere Verbleibewahrscheinlichkeit auch älterer Beschäftigter im Betrieb, je weniger Friktionen die bisherige Erwerbshistorie aufweist (vgl. Schasse (1991) und Bender et al. (2000)). So wird vermutet, dass sich Personen mit eher kontinuierlichem Erwerbsverlauf tendenziell auf Positionen wiederfinden, die ebenfalls eine weiterhin lückenlose Weiterführung der Erwerbstätigkeit erwarten lassen. Für sie dürfte daher das Risiko eines frühzeitigen Austritts aus dem Erwerbsleben sinken. Dieses vermutete Zusammenspiel unbeobachteter Personen- und Betriebsheterogenität könnte daher auch zur Erklärung dieser Schätzwerte beitragen.

Die Variable deutsch gibt an, ob die untersuchte Person die deutsche Staatsbürgerschaft besitzt. Sie geht auch gekreuzt mit einer Reihe von Altersdummies in die Regression ein und lässt so ein Altersprofil der Effekte zu. Deutliche Unterschiede lassen sich hier zwischen Ost und West ausmachen. In Westdeutschland ist ein für deutsche Männer schwach signifikanter Anstieg der Hazardrate zu beobachten, der sich in der höchsten Altersgruppe über 64 Jahren in sein Gegenteil verkehrt. Ab dann erst laufen westdeutsche, männliche Staatsbürger eine deutlich geringere Gefahr, aus dem Erwerbsleben auszuscheiden als ihre nicht-deutschen Kollegen. Für die Frauen sind die Effekte meist insignifikant und nicht eindeutig. Es lässt sich nur spekulieren, ob dieses Resultat auf einer mangelhaften Kontrolle bspw. von Vermögenswerten beruht. 
Dieses Teilergebnis deckt sich mit den wenigen Studien zu dem Thema: Antolin und Scarpetta (1998), die in ihrer SOEP-Daten-Untersuchung für Westdeutschland keine Unterscheidung von Männer und Frauen oder bestimmter Altersgruppen vornehmen, weisen ebenfalls auf die höhere Wahrscheinlichkeit von Deutschen hin, früher aus dem Erwerbsleben auszuscheiden.

In den neuen Ländern wirkt sich dagegen die deutsche Staatsangehörigkeit deutlich risikomindernd aus. Dies gilt für beide Geschlechter gleichermaßen. Diese Unterschiede lassen sich womöglich durch die Unterschiedlichkeit der jeweiligen Referenzgruppe und ihren Erwerbsbedingungen im Untersuchungszeitraum erklären.

Für die Schätzung des Einflusses der beruflichen Stellung wurde die Referenz „Angestellter“ gewählt. Gegenüber dieser erweisen sich in Westdeutschland insbesondere die Arbeiter als resistent vor dem Austritt, wohingegen Meister einem höheren Risiko ausgesetzt sind. Für Ostdeutschland lässt sich feststellen, dass einfache, männliche Arbeiter eine höhere Hazardrate aufweisen als Angestellte. Dieser Umstand ist möglicherweise der Deindustrialisierung in den neuen Ländern im Beobachtungszeitraum zuzuschreiben, die die Arbeitsmarktsituation dieser Gruppe negativ beeinflusst hat.

Auffällig sind für beide Landesteile und Geschlechter die jeweils hochsignifikanten Werte für Teilzeitbeschäftigung, die schon deskriptiv ermittelt wurden. Gerade wenn nur eine geringe Anzahl von Stunden wöchentlicher Arbeitszeit geleistet wird, verringert dies die geschätzte individuelle Hazardrate deutlich. Vermutlich waren hierbei Personen ausschlaggebend, die zusätzlich zur Altersrente hinzuverdienen und somit erst später als ihre Altersgenossen aus dem hier so definierten Erwerbsleben ausscheiden.

Die Personeninformationen enthalten eine Qualifikationsvariable, die schulische und berufliche Ausbildung miteinander kombiniert. Gegenüber der Referenz „Hochschulabschluss“ sind alle anderen - und damit formal niedrigeren - schulischen Bildungsabschlüsse mit einer erhöhten Hazardrate verbunden. Damit bestätigt sich der schon in der deskriptiven Analyse vermutete, schützende Einfluss akademischer Bildung vor einem frühzeitigen Erwerbsaustritt. Dies gilt allerdings nicht für Personen, die eine Fachhochschule abgeschlossen haben. Für sie werden überraschend hohe Hazard ratios ermittelt. Auffällig ist auch, dass - allerdings nur in Westdeutschland - bei identischem Schulabschluss eine abgeschlossene Berufsausbildung mit einer höheren Hazardrate verbunden ist.

Die Ergebnisse decken sich mit denen von Wübbeke (2005b, S. 89), die ebenfalls auf eine austrittsverzögernde Wirkung formaler Bildungsabschlüsse hinweist.

Als letzte der persönlichen Variablen wurden monetäre Einflüsse kontrolliert, die auch mit der Ruhestandsentscheidung in Verbindung stehen. Neben dem Tagesentgelt, das wieder auch gekreuzt mit der Altersangabe ab 59 Jahren in die Schätzung mit eingeht, wurde auch das kumulierte sozialversicherungspflichtige Einkommen als Regressor aufgenommen. Dabei zeigen die Ergebnisse für alle Koeffizienten höchste Signifikanz über die Geschlechter und Lan- 
desteile hinweg. Abb. 18 zeigt das Altersprofil der geschätzten Hazard ratio für das Tagesentgelt.

Abb. 18: Hazard ratio der Variablen „Tagesentgelt in Euro“ im Altersprofil

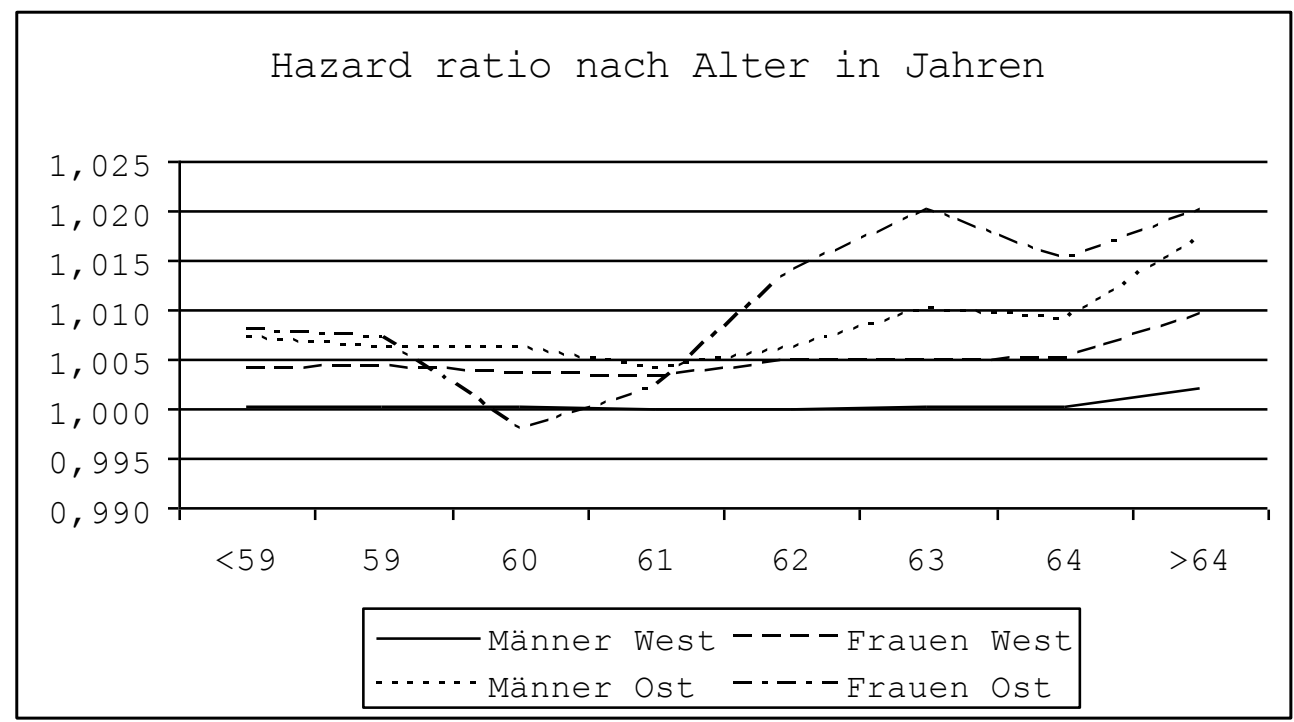

Quelle: LIAB, eigene Berechnungen

Dabei zeigt sich, dass nahezu unabhängig von Alter, Geschlecht und Wohnort die Hazardrate mit zunehmendem Lohn steigt. Erkennbar ist auch, dass der Effekt mit zunehmendem Alter stärker wird. Dies ließe sich als Ausdruck der Nachfrageseite interpretieren, die hoch entlohnte Beschäftigte zum Austritt drängt. Andererseits ließe sich eine Dominanz des Einkommenseffektes in der intertemporalen Arbeitsangebotsentscheidung ausmachen. Eine weitere mögliche Interpretation greift für den Fall, dass ein Arbeitsverhältnis zu Ende geht und ein Arbeitssuchender geringere Wieder- bzw. Weiterbeschäftigungschancen aufweist auch aufgrund der für Ältere ohnehin höheren Anspruchslöhne. ${ }^{58}$

Damit stimmen die Ergebnisse mit der Vorgängerstudie von Antolin und Scarpetta (1998, S. 29) überein, die zu einem positiven Einfluss des Lohnes auf das Vorziehen des Verrentungszeitpunktes kommen.

Die Summe der sozialversicherungspflichtigen Einkommen soll zum einen die zu erwartende Rentenhöhe und damit die Vorteilhaftigkeit einer Austrittsoption annähern. Zum anderen stellt sie eine Proxy-Variable für die Vermögens- und Alterssicherungspositionen der Personen dar. Beide Deutungen lassen vermuten, dass mit zunehmendem Wert der Variablen die Austrittsentscheidung früher gefällt wird. Umso verwunderlicher erscheint es daher, dass alle Teilschätzungen zu einer Hazard ratio von kleiner eins kommen. Dies lässt sich theoretisch nur schwer erklären, da sich dies beim Arbeitsangebot der Älteren in einem frühzeitigen Aus-

\footnotetext{
${ }^{58}$ Auf den Umstand, dass Anspruchslöhne mit dem Alter steigen, weist beispielsweise Christensen (2003) hin, der die Bestimmungsgründe der Höhe von Reservationslöhnen anhand von SOEP-Daten allerdings ausschließlich für Westdeutschland und die Bis-58-Jährigen untersucht.
} 
tritt und damit einer höheren Hazardrate niederschlagen müsste. Empirisch ließe sich allenfalls argumentieren, dass wieder eine ungenügende Datenbasis Ursache des unerwarteten Wertes der Hazard ratio sein könnte: So kann der möglicherweise ungenügend kontrollierte Gesundheitszustand mit dem kumulierten Einkommen korreliert und die Ergebnisse in der beobachteten Weise verzerrt sein.

Allerdings bestätigen sich hier frühere empirische Studien, die ebenfalls zu dem Schluss kommen, dass mit zunehmendem Vermögen die Austrittsentscheidung später gefällt wird (vgl. z.B. Antolin und Scarpetta (1998), S. 14). Dagegen widerspricht diese Aussage den in A 1.2.1.2 erwähnten Ergebnissen von z.B. Börsch-Supan und Berkel (2004).

\section{A 5.2 Einfluss von betrieblichen Variablen}

Hauptaugenmerk liegt auf der Bestimmung betrieblicher Effekte auf die Austrittsentscheidung. Der folgende Abschnitt beschäftigt sich mit diesen Variablen und ihren geschätzten Einflüssen.

\section{A 5.2.1 Institutionelles}

Wie in A 1.2.4 beschrieben, sind Unternehmen von einigen Institutionen in ihrer Weiterbeschäftigungsentscheidung reglementiert oder beeinflusst. In dieser Arbeit konnten Tarifbindung, die Existenz eines Betriebsrates und die Geltung des KSchG als solche Faktoren in das ökonometrische Modell einfließen.

Für die Bindung eines Betriebes an einen Branchentarifvertrag und die Existenz eines Hausbzw. Firmentarifvertrages kommen die Schätzungen zu teilweise hochsignifikanten Ergebnissen. In allen Teilschätzungen ist für die Variable „Branchentarifvertrag“ ein signifikant positiver Effekt zu beobachten. So erhöht das Vorhandensein eines Brachentarifvertrages die Hazardrate westdeutscher Männer um nahezu 20\%; für die anderen Schätzungen betragen die Werte zwischen 7,7 und 15,7\%. Auch der Haustarifvertrag trägt meist zur Erklärung des Austrittsverhaltens bei. Hier erreichen die Koeffizienten allerdings nicht so hohe Werte.

Es lässt sich vermuten, dass dieses Ergebnis auf die in vielen Tarifverträgen festgehaltenen Bestimmungen zur Gewährung von Altersteilzeit seitens des Betriebes zurückzuführen ist. Ein Brancheneffekt kann für dieses Ergebnis nicht verantwortlich gemacht werden, da auch für den Wirtschaftszweig kontrolliert wurde. Allerdings muss hier einschränkend erwähnt werden, dass es sich bei der Tarifbindung um eine endogene Kontrollvariable handelt, da es im Ermessen der Betriebe steht, einem Arbeitgeberverband beizutreten. Dies könnte die Ergebnisse verzerrt haben.

Gleiches lässt sich über das Vorhandensein eines Betriebsrates aussagen. Wieder ließen sich für alle untersuchten Gruppen austrittsbeschleunigende Einflüsse berechnen. Bei Existenz eines Betriebs- bzw. Personalrates stieg c.p. die Hazardrate um 12 bis 16 Prozent. Anders als es die Studien von Beckmann (2002) und Boockmann und Zwick (2004) zum Einfluss eines Betriebsrates vermuten lassen, konnte hier für die Mehrzahl der untersuchten Personen ein 
verzögernder Einfluss auf den Austritt nicht gefunden werden. Im Gegenteil unterstützen die Schätzungen Vermutungen und Ergebnisse von Wübbeke (2005), nach denen Mitbestimmungsorgane Frühverrentungen gegenüber Kündigungen Jüngerer als sozial verträglicher erachten und ihren Einfluss dahin gehend ausüben könnten.

Auch die Vermutung, dass der Kündigungsschutz und insbesondere die in $\S 1$ Abs. 3 KSchG festgehaltene Sozialauswahl den Rückzug aus dem Arbeitsmarkt verzögern würde, können hier nicht empirisch bestätigt werden. In diesem Fall brachten alle Schätzungen hohe empirische Signifikanzniveaus. Dies geht einher mit den Ergebnissen in Kapitel C, in dem ebenfalls keine Beschäftigungs- und Einstellungswirkungen für die Lockerung von Kündigungsschutzregeln nachgewiesen werden.

Zusammenfassend lässt sich sagen, dass die Sozialpartner - sowohl auf Verbands-, wie auf Betriebsebene - nicht zu einem Verbleib Älterer auf dem Arbeitsmarkt beitragen. Stattdessen scheinen sie ältere Beschäftigte aus dem Erwerbsleben zu drängen.

\section{A 5.2.2 Anpassungskosten}

Auch sich ändernde Anforderungen an Art und Umfang der Produktion im Betrieb können dazu führen, dass Älteren der Ausstieg nahe gelegt wird. Wie diese Anpassungen vonstatten gehen und ob dabei besonders Ältere betroffen sind, kann auch davon abhängen, welche Alternativen zur Entlassung von Älteren dem Betrieb zur Verfügung stehen und zu welchen Kosten diese anderen Anpassungsstrategien umsetzbar sind.

Zunächst wurde für die Umsatzerwartungen für das jeweils kommende Geschäftsjahr kontrolliert. Den dann folgenden Variablen ist gemein, dass sie als Substitute für die Anpassung an eine verringerte Arbeitsnachfrage nach Älteren dienen können und damit dazu geeignet sind, die Übergangswahrscheinlichkeit abzudämpfen.

Die erwartete Umsatzentwicklung für das laufende Jahr wirkt in der vermuteten Richtung. Alle geschätzten Parameter sind signifikant und lassen den Schluss zu, dass mit zunehmend optimistischer Einschätzung der Geschäftsentwicklung die Wahrscheinlichkeit eines Übergangs für Ältere sinkt. Mit jedem weiteren Prozentpunkt positiver Veränderung der Umsatzerwartungen sinkt in den einzelnen Teilschätzungen die Hazardrate c.p. um rd. 0,6 bis 1,0 Prozent und verringert damit die Austrittswahrscheinlichkeit in den folgenden Jahren. Allein dieses Zwischenergebnis zeigt, dass Verrentungsprozesse in der betrieblichen Personalpolitik als Anpassungsinstrument genutzt werden.

Der Anteil der Auszubildenden, deren Übernahme eine Alternative zur fortgesetzten Beschäftigung der 50-Jährigen und Älteren darstellt, übt in jeder der Teilschätzungen einen signifikanten, positiv von eins verschiedenen Einfluss auf die Hazardrate aus. Dies deutet auf einen substitutiven Zusammenhang zwischen den beiden Beschäftigtengruppen hin. Die Schätzergebnisse sind beachtlich: Steigt der Anteil Auszubildender um einen Prozentpunkt, steigt auch die Hazardrate um die zwei Prozent, für westdeutsche Männer beträgt der Wert sogar knapp fünf Prozent. Dies steht im Einklang mit den Ergebnissen von Boockmann und Zwick (2004), 
die einen negativen Einfluss des Anteils der Auszubildenden auf den Anteil der Älteren im Betrieb feststellten. Auszubildende scheinen damit die Älteren aus dem Betrieb zu verdrängen.

Der Anteil der befristet Beschäftigten übt einen deutlich positiven Einfluss auf die Hazardrate von Männern und Frauen aus. Allerdings sind die ermittelten Hazard ratios nicht so hoch wie im Falle des Anteils der Auszubildenden. In Westdeutschland erhöht sich die Hazardrate um 0,3 Prozent für Männer, in Ostdeutschland liegen die Werte bei rd. zwei Prozent. Anstatt dass befristete Verträge als Puffer dienen und damit auch die Beschäftigungssicherheit der älteren Kernbelegschaft wahren, scheinen sie in einem Konkurrenzverhältnis zu Weiterbeschäftigung Älterer zu stehen.

In der Gruppe der sonstigen Beschäftigten sind alle anderen Mitarbeiter des Betriebes, seien es Praktikanten, freie Mitarbeiter oder Leiharbeitskräfte zusammengefasst. Ihr Anteil trägt zur Erklärung des Erwerbsverlaufes Älterer nicht bei. Dies deutet darauf hin, dass zwischen ihnen und der in dieser Variablen erfassten Randbelegschaft keine Substitutionsbeziehung vorliegt, sodass sich die beiden Gruppen nicht gegenseitig ersetzen lassen.

Zusammenfassend lässt sich sagen, dass die Arbeitsmarktaussichten Älterer auch von kurzfristigen Geschäftserwartungen des beschäftigenden Betriebes abhängen und die untersuchte Gruppe daher als Manövriermasse dient bei der Anpassung an Änderungen auf Gütermärkten. Die Älteren stehen dabei in Konkurrenz mit anderen Randgruppen der Belegschaft.

\section{A 5.2.3 Altersstruktur}

Für die Altersstruktur des Betriebs ergeben sich hoch signifikante Einflüsse: sowohl der Anteil der Jüngeren (Bis-30-Jährigen) als auch derjenige der Älteren (Über-50-Jährigen) im Betrieb wirken sich verzögernd auf den Austrittstermin der Personen in der Stichprobe aus. Die Ergebnisse sind in Abb. 19 veranschaulicht.

Für den Anteil der Über-50-Jährigen gilt, dass mit Zunahme um einen Prozentpunkt die Hazardrate um bis zu vier Prozent sinkt. Besonders die Werte für Frauen der ältesten Gruppen profitieren von einem hohen Anteil ebenfalls älterer Kollegen. Je nach Alter, Geschlecht und Herkunft liegt der Effekt i.d.R. zwischen ein und zwei Prozent. 
Abb. 19: $\quad$ Hazard ratio des Anteils Über-50-Jähriger [in \%] nach Alter [in Jahren]

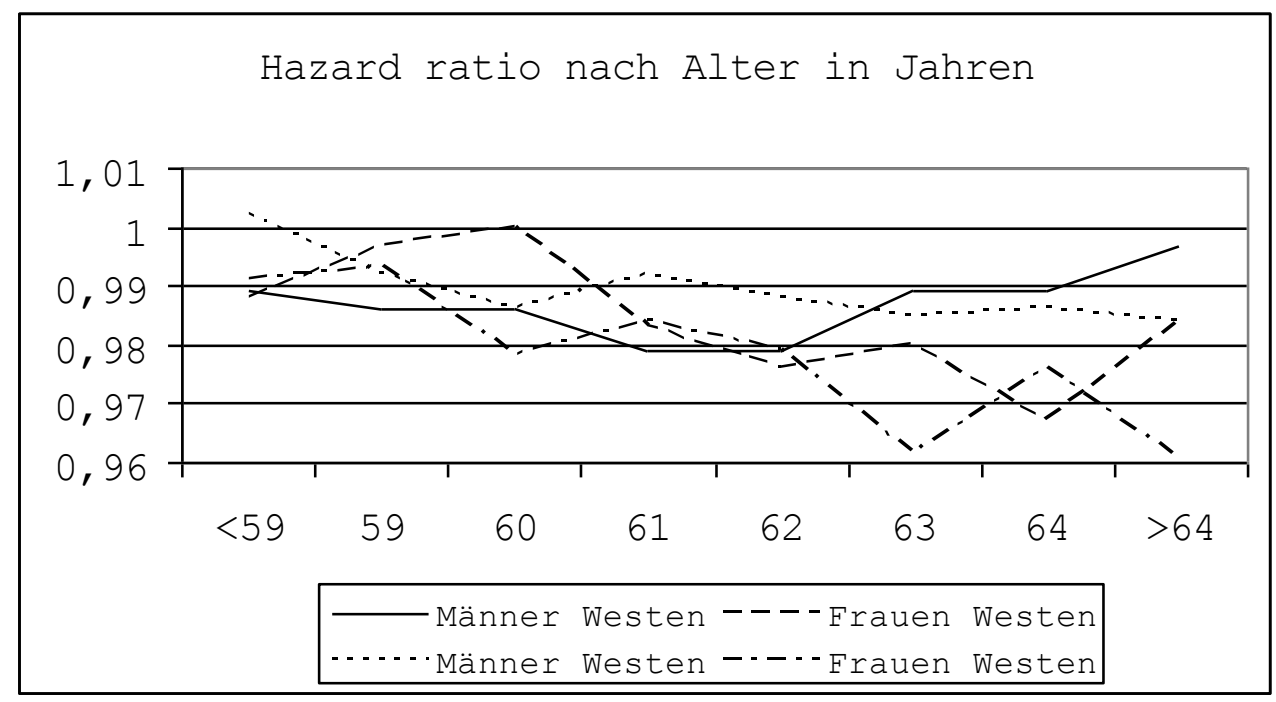

Quelle: LIAB, eigene Berechnung

Dieser Befund deckt sich mit Erkenntnissen der Studie von Blau und Shvydko (2007), die allerdings mit Selbstselektionsprozessen der Beschäftigten für ihre Resultate verantwortlich machen. Ein hoher Anteil Älterer kann als Indiz dafür angesehen werden, dass im Betrieb altersgerechte Arbeitsbedingungen vorherrschen und besonders arbeitswillige Ältere den Arbeitgeber deshalb bewusst gewählt haben. An dieser Stelle wird deutlich, dass die Ergebnisse nur bedingt kausal zu interpretieren sind.

Abb. 20: Hazard ratio des Anteils Bis-30-Jähriger [in \%] nach Alter [in Jahren]

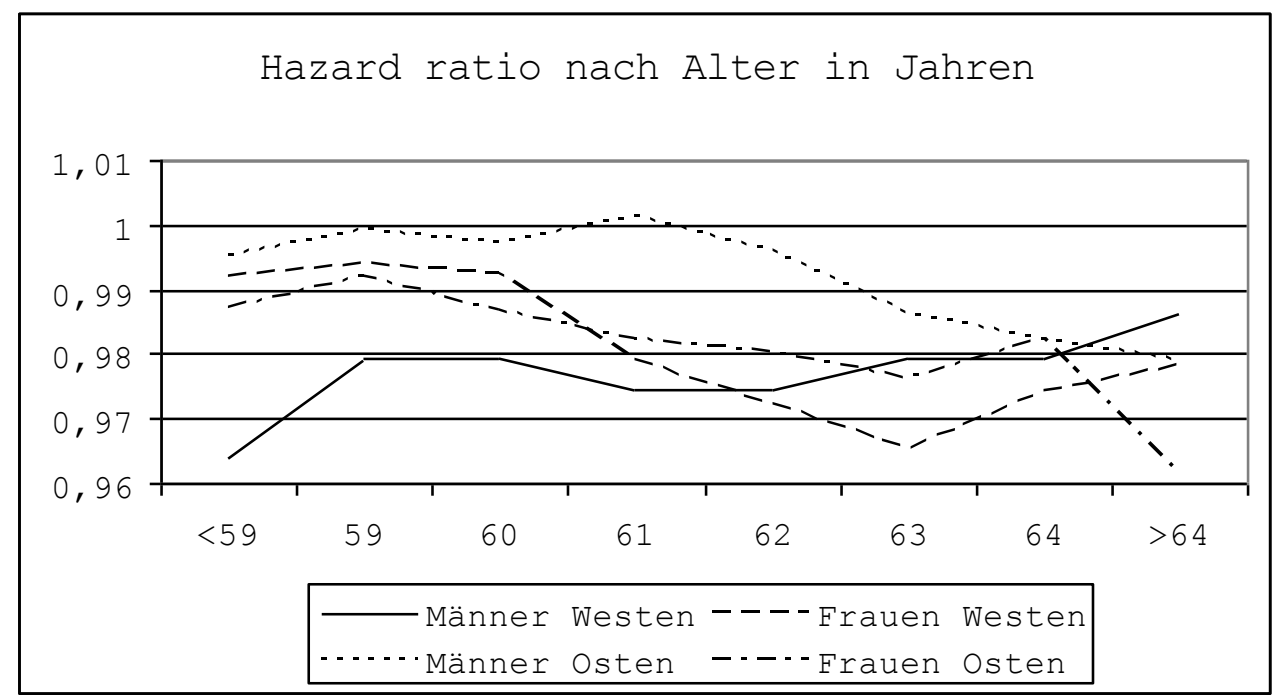

Quelle: LIAB, eigene Berechnung

Ähnlich wirkt auch ein erhöhter Anteil Jüngerer. Steigt dieser um einen Prozentpunkt, dann sinkt im Gefolge die Hazardrate beispielsweise westdeutscher Männer um 3,6 Prozent. Auch hier lässt sich für alle untersuchten Gruppen ein beschäftigungsverlängernder Effekt beobachten. Auffällig ist, dass mit zunehmendem Alter die gemessenen Einflüsse stärker werden. 
Insgesamt lässt sich dieses Teilergebnis als Komplementarität von jüngeren Arbeitskräften mit den Über-50-Jährigen interpretieren und bestätigt damit den Eindruck, der sich in der deskriptiven Analyse ergeben hatte. Den Schluss, dass Ältere durch die Einstellung Jüngerer verdrängt würden, was beispielsweise durch das Instrument der Altersteilzeit lange Zeit auch politische Unterstützung genoss, lassen die Daten nicht zu. Eher scheinen die Älteren von der Zusammenarbeit zu profitieren. Dieses Ergebnis ist im Kontrast zu den oben dargestellten Wirkungen des Anteils der Auszubildenden zu sehen, die i.d.R. ebenfalls in diese Altersgruppe fallen. Möglicherweise steht hier der Einfluss der Altersteilzeitregelung im Vordergrund, die den Bezug der Beihilfen an die Übernahme von Auszubildenden koppelte, deren hoher Anteil dann als ein Indiz für ein bevorstehendes Erwerbsende der Älteren darstellt.

Vorsicht ist allerdings bei der Interpretation der Koeffizienten allein schon deshalb angebracht, weil der Altenanteil gerade in Kleinbetrieben mit nur wenigen Personen dieser Jahrgänge stark endogen sein kann. Die hochsignifikanten Ergebnisse geben allerdings Anlass zur Hoffnung, dass das Ergebnis auch bei Beseitigung dieser Endogenität Bestand hätte.

\section{A 5.2.4 Technischer Wandel}

Um die Wirkungen technischen Fortschritts auf die Beschäftigungsaussichten von Älteren zu überprüfen, sind der technische Stand der Anlagen, die Frage, ob im vergangenen Jahr Investitionen in Kommunikationstechnik vorgenommen wurden, und der Investitionsanteil ins Modell aufgenommen worden. Während sich in der ersten Variablen das Investitionsgeschehen der vergangenen Jahre abbilden lässt, decken die letzten beiden Merkmale Angaben zu aktuellen Veränderungen im Betrieb ab. Um einen möglichen Altersverlauf der Effekte erfassen zu können, gingen neben den Dummy-Variablen zum Zustand der Ausstattung auch Interaktionsterme mit dem Alter der Beschäftigten beginnend mit dem Alter 59 Jahre ein. Daraufhin wurden aus Gründen der Übersichtlichkeit die beiden ersten Ausprägungen der ursprünglich als 5er-Skala erhobenen Frage zu der Dummy-Variablen „neu“, die beiden letzten zu „alt“ zusammengefasst. ${ }^{59}$

\footnotetext{
${ }^{59}$ Dieses Vorgehen ist angelehnt an die Arbeit von Boockmann und Zwick (2004), die darauf hinweisen, dass eine solche Zusammenfassung sich als wenig problematisch herausgestellt hat (vgl. Boockmann und Zwick (2004), S. 57).
} 
Abb. 21: Hazard ratio der Variablen „neue Technik“

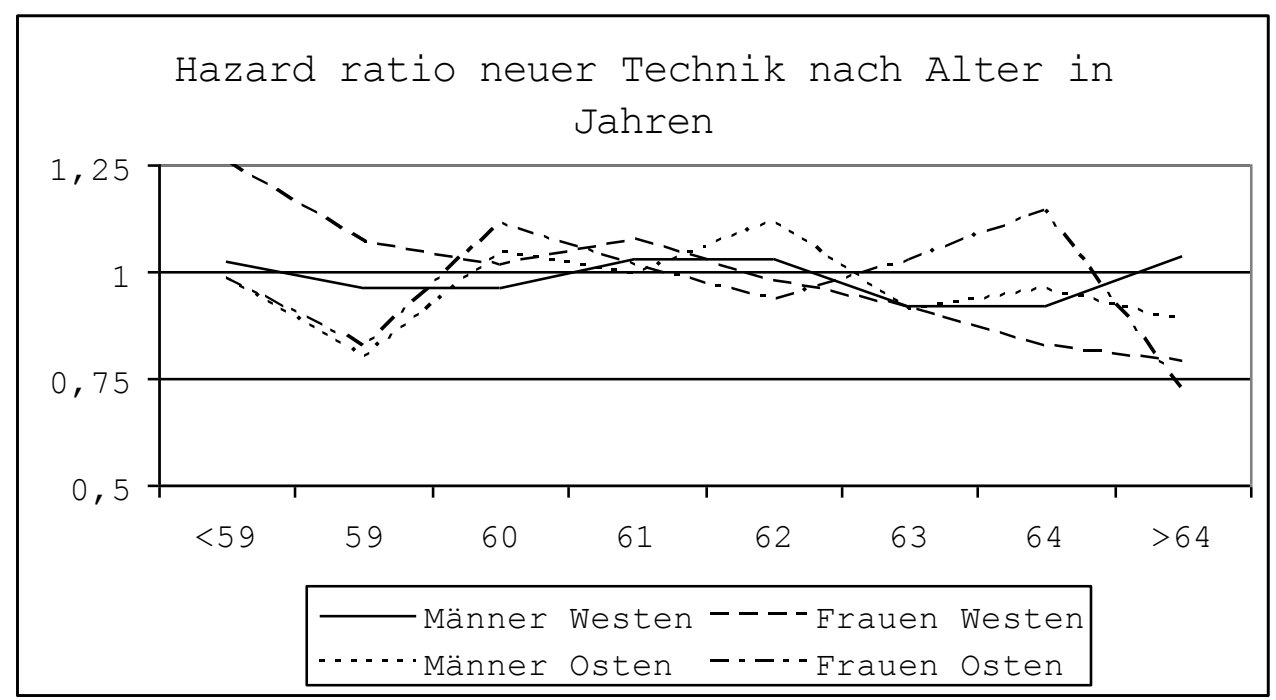

Quelle: LIAB, eigene Berechnungen

Die Ergebnisse für den Zustand der technischen Anlagen entkräften zumindest teilweise die Befürchtungen, wonach Ältere die Verlierer technischer Neuerungen im Betrieb seien: Die zugehörigen Koeffizienten und die geschätzten Effekte deuten - sofern sie signifikant sind im Gegenteil darauf hin, dass eine moderne, technische Ausstattung des Betriebes eine stabilisierende Wirkung auf die Beschäftigung Älterer ausübt. Besonders deutlich wir dies bei den Schätzungen für die Männer. Einzig für westdeutsche Frauen greift dieser Effekt erst ab einem Alter von 62 Jahren. Ansonsten gilt, dass neue technische Ausstattung des Betriebes die Hazardrate des Übergangs senkt, wohingegen die Ausprägungen „,veraltet“ und „,völlig veraltet" ein höheres Übergangsrisiko nach sich ziehen. Für ostdeutsche Frauen sind keine signifikanten Effekte auszumachen.

Abb. 22: Hazard ratio der Variablen „alte Technik“

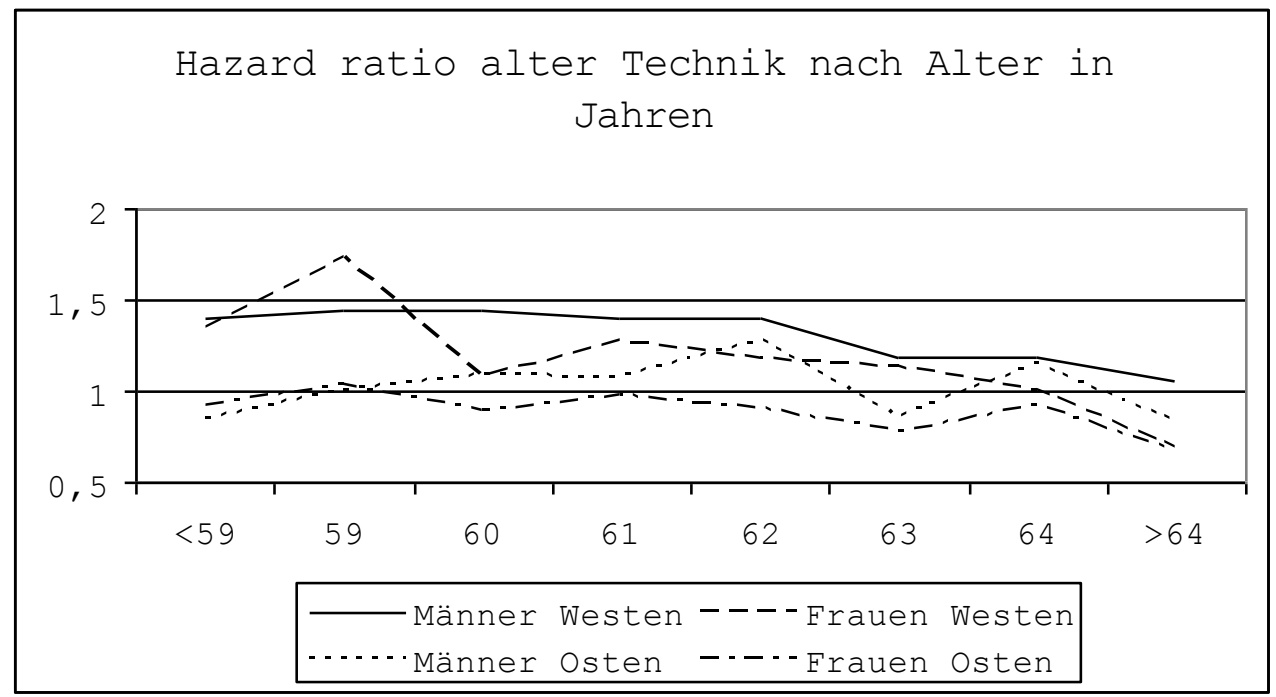

Quelle: LIAB, eigene Berechnungen 
Der Einfluss moderner Informations- und Kommunikationstechnik ließ sich nur in den Erwerbsbiografien der ostdeutschen Männer signifikant nachweisen. Dort geht der Umstand, dass der beschäftigende Betrieb im zurückliegenden Geschäftsjahr Investitionen in EDV getätigt hatte, c.p. mit einer um knapp 9\% geringeren Hazardrate (Hazard ratio: 0,914) einher. Auch für Westdeutschland deuten die Befunde in diese Richtung, lassen sich aber nicht statistisch sicher belegen. Wieder bestätigen sich die Befunde aus der deskriptiven Betrachtung.

Auf Investitionen allgemein lässt sich dieser Befund allerdings nicht übertragen. Die zugehörigen Parameter sind meist insignifikant. Einzig für die Frauen in Ostdeutschland kann eine erhöhte Austrittsrate aus der Erwerbstätigkeit nachgewiesen werden, wenn der Investitionsanteil des Unternehmens im jeweils vergangenen Jahr hoch war. Offensichtlich handelt es sich hier um eine Gruppe, der technischer Fortschritt in der kurzen Frist nicht zugute kommt.

Einschränkend muss allerdings darauf hingewiesen werden, dass Matching-Prozesse auf dem Arbeitsmarkt für diese Ergebnisse mit verantwortlich sein könnten. Es könnten sich nämlich Personen, die neuer Technik gegenüber aufgeschlossen sind, solche Arbeitgeber gesucht haben, die diese Technik in ihren Betrieben einsetzen. Hier kann also das Problem personeller unbeobachteter Heterogenität eine Rolle spielen.

Dennoch deuten die Ergebnisse dieses Variablenkomplexes zusammengefasst darauf hin, dass sich der Einzug moderner Technik - auch der EDV und Kommunikationstechnik - nicht negativ auf die Resterwerbsbiografien der untersuchten Personen auswirkt. Im Besonderen die langfristigen Effekte - wie sie im Zustand der technischen Anlagen zum Ausdruck kommen erwiesen sich sogar als beschäftigungsverlängernd. Dies wurde vor allem deutlich an den signifikant höheren Austrittsquoten von Personen in Betrieben mit veralteter oder sehr veralteter Ausstattung. In der kurzen Frist ließen sich i.d.R. keine Reaktionen von Investitionen auf die Erwerbsaussichten der Älteren feststellen. Die These vom ,age-biased technological change' lässt sich anhand dieser Resultate nicht untermauern.

Die von Bartel und Sicherman (1993) aufgestellte These, nach der beständig hohe Änderungsraten dagegen die Erwerbsdauer verlängern, bestätigt sich in den Ergebnissen zum Zustand der technischen Anlagen. So finden sich in den Ergebnissen deutliche Anzeichen dafür, dass sich technischer Wandel in der langen Frist günstig auf die Arbeitsmarktaussichten Älterer auswirkt.

Die von Bertschek (2004) formulierte These, dass sich der Einzug von IKT in den Arbeitsalltag nachteilig für die Erwerbsposition Älterer auswirkt, konnte hier nicht bestätigt werden. Vielmehr konnten für Westdeutschland die Ergebnisse von Schleife (2006) untermauert werden. Computernutzung im Betrieb übt keinen signifikanten Einfluss auf das Erwerbsverhalten Älterer aus.

\section{A 5.2.5 Sonstige betriebliche Kontrollvariablen}

Bei der Schätzung des Einflusses der Branchenzugehörigkeit erwiesen sich gerade diejenigen Wirtschaftszweige, in denen körperliche Arbeit vorherrscht, als beschleunigend für den Er- 
werbsaustritt. Dies betrifft die Branchen „Agrar, Energie, Bergbau, Wasser“, aber auch den Bausektor und deckt sich mit den Ergebnissen von Wübbeke (2005b, S. 87). Die den Dummy-Variablen zugehörigen Hazard ratios sind fast durchgängig signifikant höher als eins und weisen damit auf ein gegenüber der Referenzkategorie des öffentlichen Dienstes auf ein früheres Austrittsalter der dort Beschäftigten. Dies bestätigt die Ergebnisse von Gustman und Steinmeier (1986) und Hurd und McGarry (1993), nach denen physisch anstrengende Tätigkeiten einen frühzeitigen Erwerbsaustritt fördern. Diese Argumentation lässt sich auch damit erhärten, dass besonders die Branchen aus dem tertiären Sektor durchweg niedrige Koeffizienten aufweisen. Zu diesem zählen i. d. R: auch die Freien Berufe. Es lässt sich erkennen, dass sie Älteren Arbeitsplätze bieten mit einer vergleichsweise langen Erwerbsperspektive.

Auffällig sind die großen Unterschiede in der Höhe der geschätzten Effekte zwischen alten und neuen Bundesländern. Während im Westen Beschäftigte fast aller Branchen früher als die Referenz „Öffentlicher Sektor“ austreten, sind es im Osten nur die Angehörigen weniger Wirtschaftszweige. Die Kontrolle der Branchenvariablen behebt zudem auch das Problem der Selektivität der Stichprobenziehung für das Betriebspanel, das eine Schichtung bezüglich der Wirtschaftszweige vornimmt.

Auch die Rechtsform des Unternehmens stellt eine weitgehend zeitinvariante Betriebsvariable dar. Die Referenzausprägung „Körperschaft öffentlichen Rechts“ gewährt in den westdeutschen Ländern nach den Regressionsergebnissen keinen Schutz vor einem vorzeitigen Erwerbsende, während in den neuen Ländern andere Rechtsformen einen signifikant beschleunigenden Effekt ausüben. Es fällt zudem auf, dass in allen Schätzungen die Variablen „Einzelunternehmen“ und „Personengesellschaft“" mit einer deutlich geringeren Hazard ratio versehen sind als die anderen Dummies. Beschäftigte in Betrieben dieser Rechtsformen laufen demnach geringere Gefahr eines frühzeitigen Ausscheidens als solche in GmbHs und anderen Kapitalgesellschaften. Man kann vermuten, dass die typischerweise nähere Beziehung zwischen Arbeitgeber und -nehmer hier eine Rolle spielt.

Für die Stellung in der Unternehmensorganisation wurde die Referenz „Niederlassung“ gewählt. Steht der Betrieb des Arbeitnehmers in der Unternehmensorganisation an anderer Stelle, wie z.B. ein Betrieb in der Unternehmenszentrale, erhöht sich die Hazardrate i.d.R. deutlich. Für alle Schätzungen gilt, dass in der Zentrale Beschäftigte c.p. früher ausscheiden.

Zudem wurde der Einfluss der Betriebsgröße kontrolliert. Die entsprechend der Ziehung der Betriebe in das Betriebspanel gebildeten Klassen-Dummies sollten mögliche Selektionseffekte aus der Schätzung eliminieren, die durch die Auswahl der Betriebe und damit der erhobenen Personen resultieren könnten.

Aus allen Teilschätzungen geht hervor, dass Beschäftigte in Kleinstbetrieben mit bis zu vier Arbeitern und Angestellten eine besonders hohe Wahrscheinlichkeit des frühen Austritts haben. Die Hazardrate steigt gegenüber der Referenzkategorie von Personen aus Betrieben mit 100 bis 199 Beschäftigten um gut $28 \%$ (ostdeutsche Männer) bis rd. 86 \% (ostdeutsche Frauen). Möglicherweise reflektiert dieses Ergebnis den Umstand, dass es in Kleinstbetrieben mit 
entsprechend wenigen verschiedenen Funktionen nur schwer möglich ist, den sich mit dem Alter ändernden Anforderungen an den Arbeitsplatz gerecht zu werden. Ein interner Arbeitsmarkt, der alternative Beschäftigungsmöglichkeiten bereithalten könnte, existiert hier nicht. Damit schränken sich die oben getroffenen Aussagen über die Freien Berufe ein, finden sich doch unter den Kleinbetrieben viele Freiberufler, die zu großen Teilen nur wenige Mitarbeiter beschäftigen.

Im weiteren Verlauf der Größenklassen ergibt sich ein für die beiden Landesteile unterschiedlicher Zusammenhang mit der Austrittswahrscheinlichkeit. So ist für Westdeutschland ein deutlich positiver Einfluss der Betriebsgröße auf die Hazardrate zu verzeichnen. Besonders für westdeutsche Männer erhöhen Großbetriebe das Risiko des vorzeitigen Austritts aus dem Erwerbsleben. Für sie liegt die Hazardrate um das gut 2,5-Fache über derjenigen der Referenzgruppe der Angestellten in Betrieben zwischen 100 und 199 Mitarbeitern. Weniger deutlich, aber von der Grundaussage ähnlich ist der Fall der westdeutschen Frauen gelagert. Für die Personen eines Großbetriebes ab 5000 Beschäftigten steigt die Gefahr des Frühaustritts um 70\% - wieder gegenüber derjenigen westdeutscher Frauen eines mittleren Betriebes. Dieses Teilergebnis deckt sich mit den Schätzungen von Wübbeke (2005b), die für Großbetriebe ebenfalls deutlich erhöhte Hazard ratios des Überganges vorfindet.

Für Ostdeutschland ist der Einfluss der Betriebsgröße - mit Ausnahme der obigen Aussagen über Kleinstbetriebe - deutlich geringer. Die meisten der Größenklassendummies sind insignifikant. Ausnahmen deuten aber ebenfalls darauf hin, dass mit zunehmender Mitarbeiterzahl der einzelne eine höhere Austrittswahrscheinlichkeit aufweist. Dies gilt für die Schätzungen der Männer wie der Frauen.

In der Gesamtschau lässt sich festhalten, dass - abgesehen von Kleinstbetrieben - die Größe des Arbeitgebers in Westdeutschland einen deutlichen und positiven Einfluss auf die Austrittswahrscheinlichkeit hat, im Osten dagegen scheint der Zusammenhang weniger bedeutsam zu sein. Zu erklären wäre dies auch mit der i.d.R. besseren Möglichkeit von Großbetrieben, Austrittsbereite mit großzügigen Abfindungsangeboten zu einem freiwilligen Ausscheiden aus dem Betrieb zu ermuntern.

Die Ergebnisse decken sich mit der gängigen Literatur zum Thema. Zwar finden Boockmann und Zwick (2004) keinen signifikanten Einfluss der (logarithmierten) Betriebsgröße auf die betriebliche Altersstruktur. Die hier vorgenommene feinere Einteilung der Größenklassen und der ermittelte nicht-monotone Zusammenhang mögen diesen Unterschied erklären.

Auch für die einzelnen Bundesländer lassen sich in Ost- wie Westdeutschland Unterschiede festmachen. Dies kann daher rühren, dass die Tarifverträge, die wie beschrieben z.B. den Übergang in die Rente regeln, sich branchen- und länderspezifisch unterscheiden, aber auch mit regionalen Unterschieden der Arbeitsmärkte, die in der Frage nach der Geschäftsentwicklung des Betriebes nicht erfasst wurde. 
In Westdeutschland wurde Niedersachsen als Referenz gewählt. ${ }^{60}$ Hier fallen Berlin-West mit einem Risikoaufschlag von gut 60 bzw. 40 Prozent für Männer bzw. Frauen sowie RheinlandPfalz und das Saarland mit einem Abschlag von knapp 20 bzw. 30 Prozent ins Auge. Dass in Ostdeutschland für alle Bundesländer kontrolliert und die Referenz „Westdeutschland“ gewählt wurde, ist dem Umstand geschuldet, dass für die Zuordnung Ost- und Westdeutschland die Personenvariable gewählt wurde, die Bundeslandvariable aber den Standort des Betriebes angibt. Die Referenzgruppe stellen hier demnach die Pendler mit ostdeutschem Wohn- und westdeutschem Arbeitsort dar. Dabei kommt zum Vorschein, dass ostdeutsche Männer, die im Westen arbeiten, früher ausscheiden als ihre in den neuen Ländern arbeitenden Kollegen. Möglicherweise ist hier die Gruppe derer für das Ergebnis verantwortlich, die zur Arbeit in den Westen gehen mussten, ihren Wohnort aber in der ehemaligen DDR beibehielten. Für die Frauen sind solche Effekte nicht festzustellen, die Hazard ratios sind insignifikant.

\section{A 5.3 Einfluss des Rentenzugangsrechts}

Um die institutionellen Regelungen des Rentenzugangs als bedeutendem Zielzustand der Abgänger aus der Erwerbstätigkeit zu erfassen, wurden Variablen gebildet, die personen- und zeitpunktspezifisch angaben, ob und unter welchen Voraussetzungen eine vorzeitige Alters bzw. Erwerbsminderungsrente möglich war. Aufgrund der oben beschriebenen unzureichenden Datenlage können diese Variablen jedoch nur näherungsweisen Charakter haben. Grundsätzlich wurden für jede Rentenzugangsform drei Variablen gebildet, die angeben, ob der Zugang ohne, mit oder gar nur mit maximalem Abschlag offen stand. Referenz bildet dabei immer der Zustand, dass die betreffende Rente noch nicht bezogen werden konnte. Fehlende Schätzergebnisse sind aufgrund von parallelen Änderungen der Gesetzeslage auf Multikollinearität zurückzuführen.

Insgesamt sind alle einbezogenen Variablen hoch signifikant und zeigen die wichtige Bedeutung der rentenrechtlichen Anreizwirkungen auf die Erwerbsbeteiligung der älteren Erwerbsbevölkerung. So plausibel die Ergebnisse in der Gesamtschau sind, umso verwirrender nehmen sich die Details der Schätzungen aus. Insbesondere die Hazard ratios für den Rentenzugang aus Altersteilzeit und Arbeitslosigkeit weisen allesamt auf einen austrittsverzögernden Effekt hin. Dabei muss allerdings das erklärte Ereignis ins Gedächtnis gerufen werden. Dieses ist nicht als Renteneintritt, sondern als Austritt aus dem Erwerbsleben definiert. Personen, die diese Zugangsart wählen, gelten zum Zeitpunkt eines möglichen Renteneintritts schon lange als ausgetreten. Für die anderen Variablen ergibt sich i.d.R. der erwartete beschleunigende Effekt.

\footnotetext{
${ }^{60}$ Daneben fungieren die vermutlich kleine Gruppe derer als Referenz, die in Westdeutschland lebt und in Ostdeutschland arbeitet.
} 


\section{A 6 Zusammenfassung der mikroökonometrischen Ergebnisse}

In diesem Kapitel A wurde erstmals auf Basis von Personendaten, die um umfangreiche Informationen des beschäftigenden Betriebs ergänzt wurden, die Austrittsentscheidung älterer Beschäftigter aus dem Erwerbsleben untersucht. Mit Hilfe der detaillierten Angaben zur betrieblichen Seite des Arbeitsmarktes konnte dabei die Nachfrageseite des Arbeitsmarktes genauer als in vorangegangenen Analysen in die ökonometrische Schätzung des Erwerbsaustritts einbezogen werden.

Bisher kam nur in wenigen Arbeiten zu dem Thema der Einfluss der betrieblichen Seite zur Geltung: Dieses Kapitel schließt damit an die Arbeiten von Wübbeke (2005a, 2005b) an, die für die Zeitspanne von 1975 bis 1995 mit Daten der IABS, ergänzt um Daten der Rentenversicherer, bereits einige betriebliche Größen in ihre Schätzungen einbeziehen konnte.

Der hier verwendete Datensatz zeichnet mit seinen Angaben über die Erwerbsverläufe von rd. 425000 auswertbaren Individuen der Jahrganges 1945 und früher ein genaues Bild des Austrittsverhaltens im Zeitraum von 1996 bis 2004. Mit seiner Hilfe lassen bisher nicht untersuchte, betriebliche Einflüsse auf das Erwerbsaustrittsverhalten ausmachen.

\begin{tabular}{|c|c|c|c|c|}
\hline & \multicolumn{2}{|c|}{ West } & \multicolumn{2}{|c|}{ Ost } \\
\hline & Männer & Frauen & Männer & Frauen \\
\hline \multicolumn{5}{|l|}{ Institutionelles } \\
\hline Tarifvertrag & - - - & - - & $-\ldots$ & - - - \\
\hline Betriebsrat & & - & $-\ldots$ & $-\ldots$ \\
\hline Geltung des KSchG & - & $\varnothing$ & $\varnothing$ & $\varnothing$ \\
\hline \multicolumn{5}{|l|}{ Anpassungskosten } \\
\hline Umsatzerwartung & +++ & +++ & +++ & +++ \\
\hline Azubis & - . & - . & - . & - - \\
\hline Befristet Angestellte & & & - - - & - . - \\
\hline Sonstige Angestellte & $\varnothing$ & $\varnothing$ & $\varnothing$ & $\varnothing$ \\
\hline \multicolumn{5}{|l|}{ Altersstruktur } \\
\hline Bis-30-Jährige & +++ & +++ & +++ & +++ \\
\hline Über-50-Jährige & +++ & +++ & +++ & +++ \\
\hline \multicolumn{5}{|l|}{ Technischer Wandel } \\
\hline Anlagen neu & + & $\varnothing$ & + & + \\
\hline Anlagen alt & - & - & - & $\varnothing$ \\
\hline Aktuelle Investitionen & $\varnothing$ & $\varnothing$ & +++ & $\varnothing$ \\
\hline Investitionen in IKT & $\varnothing$ & $\varnothing$ & $\varnothing$ & - - - \\
\hline
\end{tabular}

Quelle: LIAB, eigene Berechnungen

Für die multivariate Schätzung dieser Einflüsse wurde getrennt für Männer und Frauen, sowie Ost- und Westdeutschland ein Cox proportional Hazardratenmodell geschätzt, das zwar auf die Kontrolle unbeobachteter betrieblicher Heterogenität verzichtet, aber zeitkonstante, betriebliche Merkmale erfasst. Tabelle 11 fasst die wichtigsten Ergebnisse dieser Schätzungen zusammen. Dabei symbolisieren Pluszeichen eine beschäftigungsverlängernde Wirkung, Minuszeichen eine -verkürzende. Die Zahl der Zeichen charakterisiert die statistische Signifikanz der Ergebnisse. 
Die Hypothese, dass institutionelle Hürden der Betriebe bei der Entlassung von Älteren das Ausscheiden dieser Personengruppe aufschieben, konnte nicht bestätigt werden. Vielmehr deuten die Ergebnisse darauf hin, dass Frühverrentungsprozesse von Sozialpartnern auf der Ebene der Tarifvertragsparteien wie auch des Betriebs- bzw. Personalrates sogar begünstigt werden. So konnte ein beschleunigender Effekt der Tarifbindung auf die Austrittsentscheidung ermittelt werden, der möglicherweise auf die dann erleichterte Wahrnehmung der Altersteilzeit durch den Versicherten zurückzuführen ist.

Auch die Regelungen des für Ältere besonders strengen Kündigungsschutzgesetzes haben nach diesen Ergebnissen - mehr dazu im folgenden Kapitel - verzögernde Wirkung auf den Austritt.

Die Annahme, dass die betriebliche Entscheidung über die Weiterbeschäftigung Älterer in starkem Ausmaß als Anpassungsinstrument des Personalbedarfs bei Nachfrageschwankungen dient, konnte allgemein bestätigt werden. Die Abhängigkeit der Austrittswahrscheinlichkeit von den Umsatzerwartungen des beschäftigenden Betriebes spricht hier eine deutliche Sprache. Andere Gruppen der Belegschaft, deren Entlassung bzw. Nichtweiterbeschäftigung möglicherweise mit geringeren Kosten verbunden wäre, wie etwa Auszubildende und befristet Beschäftigte schützen Ältere nicht vor dem Risiko einer Beendigung des Erwerbslebens. Im Gegenteil steigert ein hoher Anteil dieser beiden Gruppen im Betrieb die Wahrscheinlichkeit eines frühzeitigen Übergangs.

Einflüsse, die von der Altersstruktur ausgehen, deuten darauf hin, dass einerseits eine ausgewogene Verteilung der Altersgruppen zu einer erhöhten Weiterbeschäftigungswahrscheinlichkeit der Über-50-Jährigen führt. So senkt ein höherer Anteil Jüngerer c.p. die Austrittswahrscheinlichkeit. Andererseits sind Beschäftigte in Betrieben mit hohem Altenanteil ebenfalls einem geringeren Risiko ausgesetzt.

Die These einer Verdrängung Älterer aus dem Erwerbsleben aufgrund sich wandelnder Qualifikationsanforderungen mit dem Einzug moderner Technik im Allgemeinen, sowie von Kommunikations- und EDV-Technik im Besonderen findet in den Schätzergebnissen keinen Widerhall. Stattdessen weisen die Resultate darauf hin, dass unterbliebene Modernisierung der technischen Anlagen in zurückliegenden Perioden die Erwerbsposition Älterer deutlich schwächt, wohingegen moderne und sehr moderne Anlagen auch den älteren Beschäftigten günstigere Beschäftigungsperspektiven bieten. Auch kurzfristig konnten - abgesehen von zwei Einzelschätzungen - keine nachteiligen Auswirkungen von technischem Wandel, wie Investitionen in IKT, festgestellt werden.

Die Ergebnisse zeigen, dass die betrieblichen Bedingungen und die Nachfrageseite des Arbeitsmarktes für Ältere deutlichen Einfluss auf das Austrittsverhalten nehmen. Eine rein angebotsorientierte Sicht vernachlässigt daher wichtige Aspekte des Übergangs aus der Beschäftigung in den Ruhestand. 


\section{B Einstellungschancen von Älteren - Wie wirkt der Schwellenwert im Kündigungs- schutz $?^{61}$}

Eine besondere, im vorigen Kapitel nur unzureichend geklärte Frage war die Wirkung des Kündigungsschutzes auf die Erwerbsposition Älterer. Eine immer wieder formulierte Hypothese ist, dass die in Deutschland geltenden Kündigungsschutzregeln die Einstellungsaussichten Älterer beeinträchtigen. In Folge auch dieser Diskussion wurde eine Reihe von Änderungen am Kündigungsschutzgesetz vorgenommen. Nachdem sich die empirischen Wirtschaftswissenschaften zunächst nur zögerlich der vieldiskutierten Thematik angenommen haben, liegen nun einige Studien zur Wirkungsweise von Kündigungsschutz vor. Allerdings fehlt bisher eine gesonderte Untersuchung zu den Auswirkungen dieser Institution auf den Arbeitsmarkt für Ältere. Dieses Kapitel B soll sich dieser Lücke annehmen.

Ein nicht nur im deutschen Kündigungsschutzrecht verankertes Detail ist der Schwellenwert seiner Anwendung. Überschreitet ein Betrieb die im Gesetz festgelegte Anzahl von Mitarbeitern, fällt er in den Geltungsbereich des KSchG und sieht sich dann - in Zeiten schwindender Nachfrage - deutlich höheren Anpassungskosten gegenüber als ein kleinerer Betrieb, für den die Regelung nicht gilt. Dies führt zum einen allgemein zu einer Erhöhung der erwarteten Kosten einer möglichen zukünftigen Entlassung und hebt damit die relativen Kosten des Faktors Arbeit. Werden diese verringert, erhöht sich c.p. die gleichgewichtige Beschäftigung. Zum anderen hindern - so die Theorie - Schwellenwerte, wie sie das gegenwärtige Recht vorsieht, kleine Unternehmen an der Einstellung weiterer Mitarbeiter und damit am Wachstum, weil mit dem Überschreiten der Schwellenwerte das Kündigungsschutzrecht unmittelbar für alle Mitarbeiter Anwendung findet. Die Grenzkosten der Einstellung dieses Mitarbeiters sind damit deutlich höher als für andere Beschäftigte. Besonders die Nachfrage nach Älteren wird erschwert, da die Gruppe einem besonderen Schutz unterliegt, wie im Folgenden noch näher zu erwähnen sein wird.

Ziel der vorliegenden Arbeit ist es zu überprüfen, ob diese zweite Hypothese insbesondere für Ältere einer empirischen Untersuchung standhält und damit die Einschränkung der Rechte von einer Vielzahl von Beschäftigten rechtfertigt. Ein Blick auf eine rein deskriptive Auswertung macht deutlich, welche weit reichende Bedeutung der Änderung des Kündigungsschutzgesetzes zukommt: Als im Januar 2004 der Schwellenwert des Geltungsbereiches von fünf

\footnotetext{
${ }^{61}$ Vorläufer dieses Kapitels sind erschienen als FFB-Diskussionspapier (Burgert (2006a)), als Zeitschriftenartikel (Burgert (2006b)) sowie als Beitrag zum Konferenzband „Freie Berufe, Einkommen und Steuern“ (Burgert (2008)). Sie sind u.a. auf der IZA summer school, dem Forschungskolloquium Freie Berufe, Mittelstand und empirische Wirtschaftsforschung an der Universität Lüneburg und auf der Konferenz "Econometrics of Labour Demand" in Mons/Belgien diskutiert worden.
} 
auf zehn sozialversicherungspflichtig Beschäftigte erhöht wurde, verloren die rd. 2,4 Mio. sozialversicherungspflichtig Beschäftigten in den nun von der Regulierung befreiten rd. 330000 Betriebe den arbeitsrechtlichen Schutz ihres Beschäftigungsverhältnisses (vgl. Abb. 23).

Abb. 23: Anzahl der sozialversicherungspflichtig Beschäftigten nach Betriebsgröße im Juni 2003

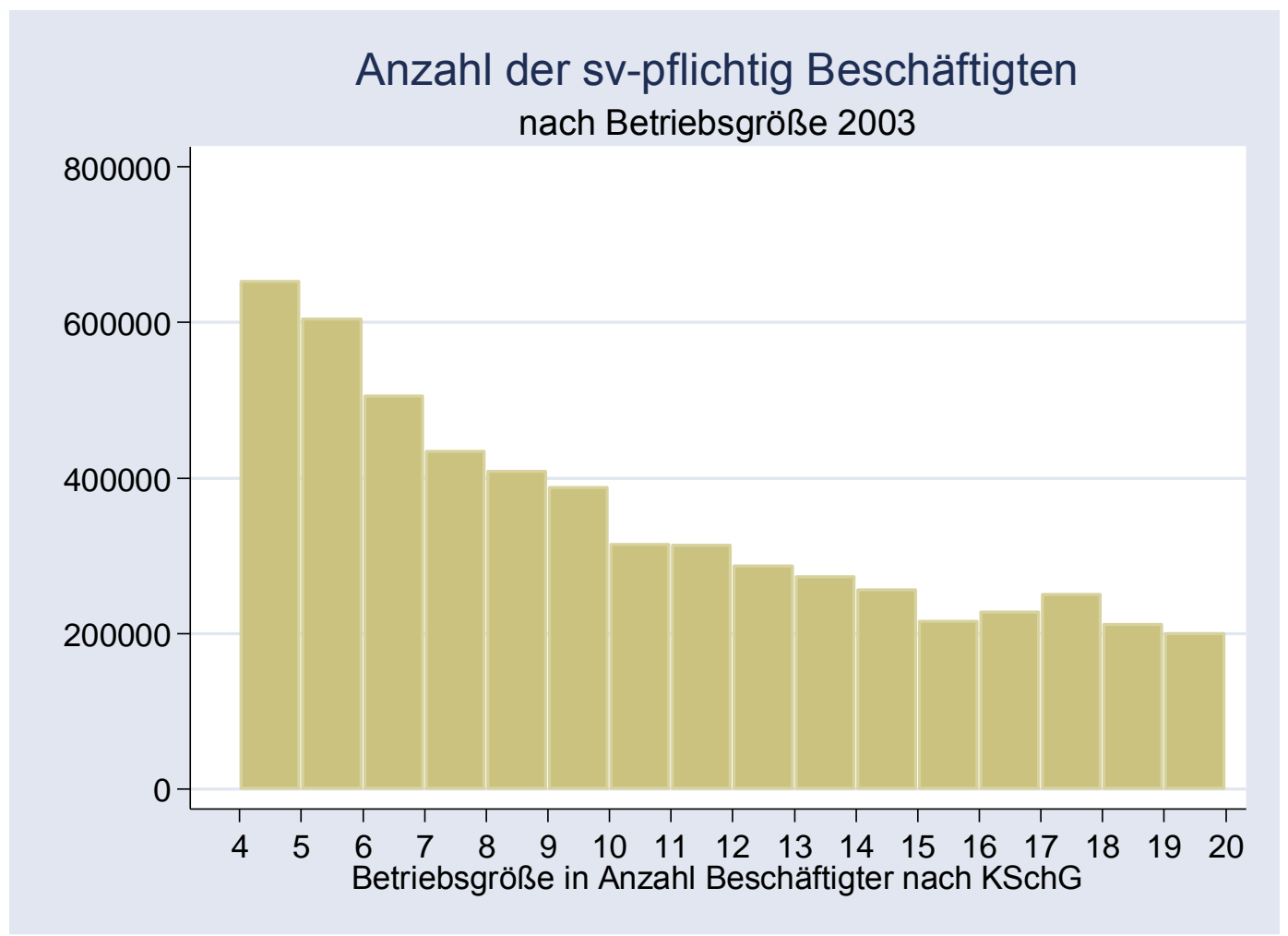

Quelle: IAB-Betriebspanel 2003, eigene Berechnungen

Zu diesem Zweck wird ein Regression Discontinuity Design (RDD)-Ansatz herangezogen, in dem die Änderungen der rechtlichen Regelungen als natürliche Experimente verwendet werden. Das IAB-Betriebspanel bietet, erweitert um Altersinformationen aus der Beschäftigtenstatistik, wiederum die Datengrundlage für die Untersuchung.

Die oben formulierte Hypothese zur Wirkung des Schwellenwertes auf das Einstellungsverhalten im Allgemeinen ist in einigen Arbeiten untersucht, für Deutschland aber nie empirisch bestätigt worden: Weder die Studie von Wagner et al. (2001) noch die von Boockmann und Hagen (2001), Verick (2004) oder Bauer et al. (2004) finden statistisch signifikante Belege für den vermuteten hemmenden Effekt. Für das betriebliche Einstellungsverhalten von Älteren im Speziellen ist die These noch nicht untersucht worden.

Dieses Kapitel ist wie folgt aufgebaut: Unterkapitel B1 beschreibt die gesetzlichen und institutionellen Regelungen zum Schutz bestehender Beschäftigungsverhältnisse insbesondere Älterer und gibt einen Überblick über die theoretische Diskussion und empirische Studien zum Thema. Im darauf folgenden Abschnitt werden die verwendeten Daten des LIAB kurz 
vorgestellt. Teil B 3 erläutert das RDD als Evaluationsmethode und seine Anwendbarkeit für die Aufgabenstellung. Im Unterkapitel B 4 werden die Ergebnisse präsentiert und abschlieBenden in B 5 daraus abzuleitende Politikempfehlungen formuliert.

\section{B 1 Kündigungsschutz in Deutschland - institutionelle Hinter- gründe}

Das Arbeitsrecht schützt bestehende Arbeitsverhältnisse zunächst unabhängig vom Alter der Beschäftigten. Allerdings sieht es für Ältere einen besonderen Schutz vor. Dazu kommen andere Mechanismen, die eine Entlassung von älteren Beschäftigten für den Arbeitgeber erschweren. Diese Regelungen werden im Folgenden dargelegt.

\section{B 1.1 Altersunabhängiger Kündigungsschutz}

Der Ablauf einer Entlassung ist in Deutschland hochgradig reguliert. Neben einer allgemeinen Gesetzgebung, die im Bürgerlichen Gesetzbuch (BGB) festgehalten ist und die technische Einzelheiten des Vorganges regelt, gilt ein spezielles Kündigungsschutzgesetz. Diese Regelung - ursprünglich eingeführt, um Arbeitnehmer vor willkürlicher Kündigung zu schützen sieht vor, dass das kündigende Unternehmen Gründe für eine Entlassung geltend machen muss. Dabei sind betriebsbedingte, personenbedingte oder verhaltensbedingte Gründe anzuführen.

Die übergroße Mehrzahl der in Deutschland ausgesprochenen Kündigungen wird mit betriebsbedingten Gründen gerechtfertigt. In diesen Fällen ist die Arbeitgeberin in der Auswahl der zu kündigenden Mitarbeiter nicht frei, sondern muss eine so genannte Sozialauswahl treffen, die im folgenden Unterkapitel näher erläutert wird.

Diese Regelungen erhöhen nicht nur die Anpassungskosten für Unternehmen. Sie machen die zukünftig anfallenden Kosten auch nahezu unberechenbar für den Arbeitgeber. Jahn und Schnabel (2003) ermitteln für 2001, dass rd. $27 \%$ aller Kündigungen, die vom Arbeitgeber ausgehen, vor Gericht verhandelt werden. Gleichzeitig fallen 75 bis $80 \%$ der Entscheidungen zugunsten des Angestellten aus. In diesem Fall kann eine Kombination aus fortgesetzter Lohnzahlung bis zur gerichtlichen Entscheidung und einer Abfindungszahlung, um den Angestellten nicht wieder einstellen zu müssen, sich leicht zu einem Jahreslohn aufsummieren. Zudem ist anzunehmen, dass viele Fälle gar nicht vor Gericht landen, sondern gegen Zahlung einer Abfindung eine Auflösung des Vertrages vorgenommen wird.

Allerdings gilt das KSchG nicht für alle Unternehmen: Kleinbetriebe, die weniger als zehn Arbeitnehmer beschäftigen, sind von dem Gesetz ausgenommen. Der Gesetzgeber hat diesen Geltungsbereich in den vergangenen Jahren mehrmals geändert. Im Mai 1996 beschloss die konservative Koalition, den Schwellenwert von damals fünf auf zehn Mitarbeiter zu erhöhen. Am 16. September 1996 passierte der Gesetzentwurf den Bundestag. Am 1. Oktober 1996 trat die Änderung in Kraft. Das Ziel dieses „Beschäftigungsförderungsgesetzes“ war es, in Zeiten von Massenarbeitslosigkeit kleinen Unternehmen ein Hindernis beim Stellenaufbau aus dem 
Weg zu räumen. Nach der darauf folgenden Bundestagswahl nahm die neue Regierung diese Änderungen umgehend wieder zurück, sodass ab 1. Januar 1999 der alte Schwellenwert von fünf Mitarbeitern wieder gültig wurde und Kleinbetriebe zwischen fünf und zehn Mitarbeitern wieder von der Regelung betroffen waren. Auch diese Ausgestaltung des Gesetzes hatte nicht lange Bestand und wurde mit Wirkung zum 1. Januar 2004 wieder in ihrer alten Gestalt mit einem Grenzwert von zehn Mitarbeitern eingeführt. Abb. 24 zeigt den zeitlichen Ablauf der Gesetzesänderungen auf.

Abb. 24: $\quad$ Zeitliche Entwicklung des Schwellenwertes im KSchG

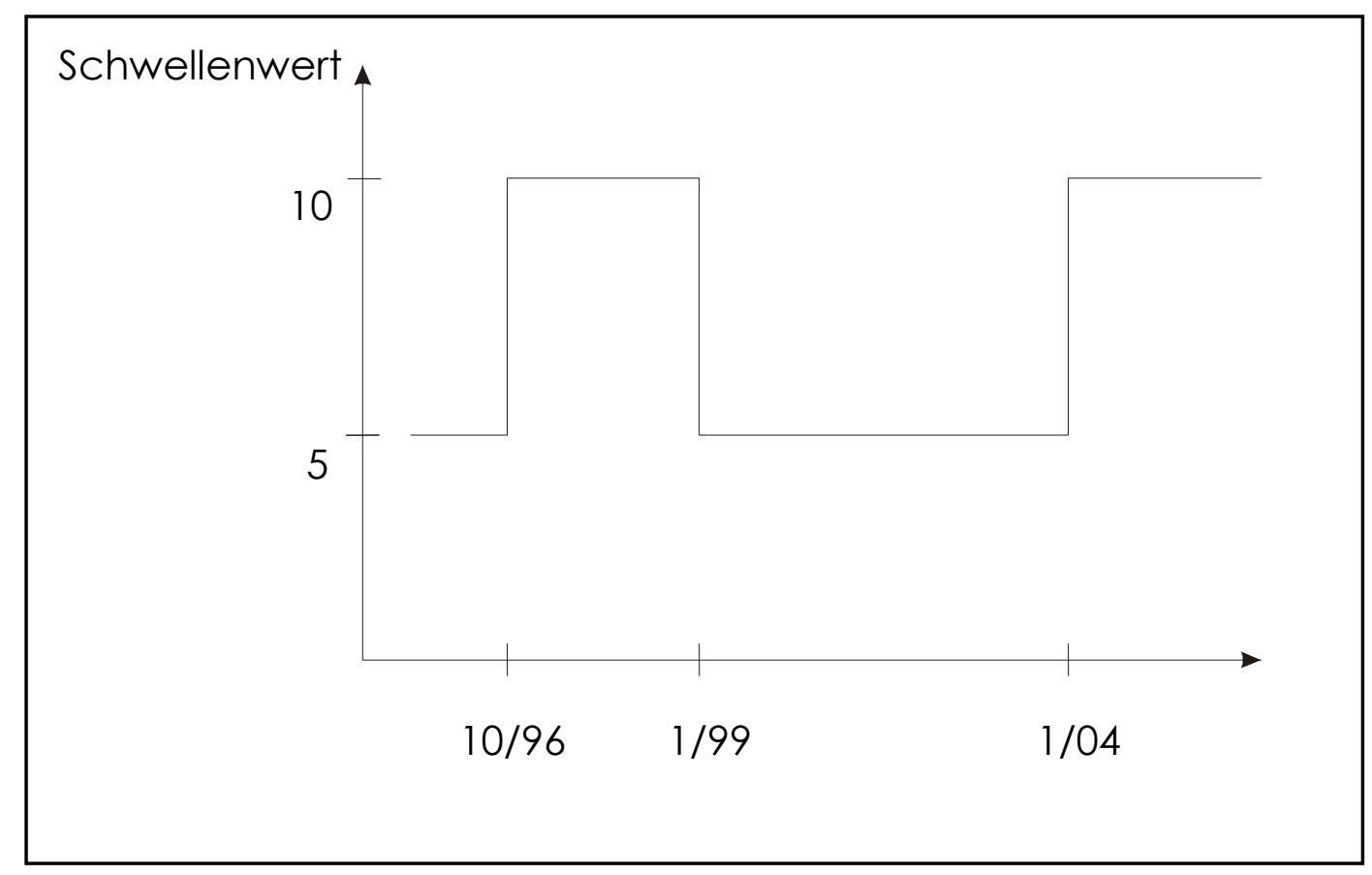

Quelle: Eigene Erstellung

Die Zahl der Beschäftigten, wie das Gesetz sie definiert, entspricht allerdings nicht der Zahl der Personen, die in einem Betreib arbeiten. Zum einen schließt sie weder Auszubildende, noch mithelfende Familienangehörige, noch den Arbeitergeber mit ein. Zum anderen zählen Teilzeitbeschäftigte nur im Ausmaß ihrer Beschäftigung: Angestellte mit bis zu 20 regulären Wochenarbeitsstunden gehen mit dem Wert 0,5 in die Berechnung der Betriebsgröße ein. Entsprechend werden Beschäftigte mit bis zu 10 (bzw. 30) Stunden wöchentlicher Normalarbeitszeit in der Woche als 0,25 (bzw. 0,75) gezählt. Befristet Beschäftigte gelten nur dann als Teil des Betriebes, wenn sie mindestens sechs Monate im Jahr dort arbeiten. Die Anzahl der Beschäftigten, die über die Anwendung des KSchG entscheidet, kann sich demnach deutlich von der Anzahl der Angestellten in einem Betrieb unterscheiden. Ein Betrieb ist dabei vom Gesetz als produktionstechnische, nicht als juristische Einheit definiert.

Anzufügen bleibt, dass nach der Kohl'schen Reform 1996 für die Beschäftigten der Betriebe zwischen fünf und zehn Beschäftigten eine Übergangsfrist von drei Jahren galt. In dieser Zeit blieben nur Neueingestellte dieser Betriebe ohne den vom Gesetz festgelegten Kündigungsschutz. Trotzdem ist zu erwarten, dass Unternehmen, die sich nach den Reformen des Geset- 
zes in den Jahren 1996 und 1998 kurz vor dem Schwellenwert wieder fanden, einen starken Anreiz hatten, auf weitere Einstellungen zu verzichten, um nicht in den Geltungsbereich des Gesetzes hineinzuwachsen.

Aus theoretischer Sicht bestehen keine Zweifel: Die Einstellung eines sechsten (bzw. elften) Mitarbeiters ist mit deutlich erhöhten Kosten in Form von erwarteten Abfindungs- und Lohnfortzahlungen verbunden. Unternehmen an der Schwelle werden diese Kosten in ihre Investitionsentscheidungen einfließen und sich davon abhalten lassen, zusätzliches Personal einzustellen. Sobald die Schwelle von zehn Beschäftigten überschritten ist, gelten nämlich für alle im Betrieb Beschäftigten die Schutzrechte des KSchG. Zudem kann die Entscheidung nicht kurzfristig durch Entlassung von Mitarbeitern rückgängig gemacht werden. Je nachdem, ob der zu Kündigende rechtliche Schritte gegen die Entscheidung einlegt und wie groß die Arbeitsbelastung der Gerichte ist, kann der Versuch, wieder unter die Schwelle des KSchG zu schrumpfen, leicht ein Jahr und länger dauern. Unter der Annahme, dass Betriebe über der Schwelle ihre Beschäftigung aufgrund des Schwellenwertes bei positiven Geschäftserwartungen nicht reduzieren, kann man deshalb davon ausgehen, dass Kleinbetriebe unter der Schwelle weniger Einstellungen vornehmen als diejenigen, die bereits über der Schwelle liegen.

Das einzige dem Verfasser bekannte theoretische Modell, das Schwellenwerte im Kündigungsschutz-recht einbezieht, ist die Arbeit von Garibaldi et al. (2003). Sie bestätigen in einem einfachen Modell die beschriebene Vermutung, dass es für einige kleine Betriebe sinnvoll ist, den Schwellenwert nicht zu überspringen, um sich so in Zeiten geringerer Nachfrage niedrigere Anpassungskosten der Arbeitsnachfrage zu sichern. Für diese Betriebe bieten sich zahlreiche Möglichkeiten, sich dem KSchG zu entziehen. Erhöhter Einsatz von Überstunden, kapitalintensivere Produktion oder eine Umstellung in den betrieblichen Make-or-BuyEntscheidungen können als Substitute zur Einstellung zusätzlicher Mitarbeiter dienen.

Diese Argumente lassen erkennen, dass es sich bei einer Einstellung um eine für den Arbeitgeber kostspielige Entscheidung handeln kann, die durch den Schwellenwert eine Zuspitzung erfährt. Unterstützt wird die These durch einen weiteren Gesichtspunkt: Neben den direkt messbaren, wenn auch nur zu erwartenden Kosten gibt die Literatur Hinweise auf weitere, für den Arbeitgeber nachteilige Anreizeffekte von Kündigungsschutzregeln. Danach reduzieren Arbeitnehmer, die einen höheren Grad an Kündigungsschutz genießen, ihren Arbeitseinsatz, da Arbeitgeber die Erfüllung von unvollständigen Verträgen nur eingeschränkt mit der Drohung einer Entlassung einfordern können. ${ }^{62}$

\footnotetext{
${ }^{62}$ Eine wachsende Anzahl von Studien belegt dieses Argument. Ichino and Riphahn (2005) zeigen anhand von Individualdaten einer italienischen Bank, dass nach dem Ablauf der Probezeit, in der Kündigungen leicht ausgesprochen werden können, die Fehlzeiten am Arbeitsplatz sprunghaft zunehmen. Weitere Arbeiten, die den $\mathrm{Zu}$ sammenhang zwischen Arbeitseinsatz und Kündigungsschutz untersuchen sind, Riphahn und Thalmaier (2001), die die oben genannten Thesen in einer Analyse von SOEP-Daten unterstützen. So finden auch sie, dass mit einem Ende der Probezeit die Fehlzeiten diskontinuierlich und signifikant steigen. Riphahn (2004) nutzt Unter-
} 
Empirische Studien über Kündigungsschutzregelungen im Allgemeinen kommen zu unterschiedlichen Ergebnissen. Während die meisten solchen Gesetzen einen beschäftigungshemmenden Effekt attestieren, kann eine Minderheit keine oder insignifikante Ergebnisse vorweisen (vgl. Addison und Teixeira 2003, S. 57ff). Die Mehrheit dieser Arbeiten basieren auf internationalen Vergleichen, für die ein Kündigungsschutzindex berechnet wird. Die bekanntesten Beispiele stellen die Studien von Lazear (1990) und der OECD (1999) dar. Allerdings schränken geringe Fallzahlen und die Problematik der Indexerstellung die Aussagekraft dieser Art der Untersuchung ein.

Nur wenige Studien stützen sich auf Mikrodaten, die individuelle Betriebsinformationen enthalten. Für Italien können Garibaldi et al. (2003) Hinweise dafür liefern, dass eine ähnliche Schwelle bei 15 Beschäftigten bremsenden Einfluss auf das Einstellungsverhalten von Betrieben ausübt. Für Deutschland präsentieren Friedrich und Hägele (1997) deskriptive Ergebnisse. Sie vergleichen den Anteil von Unternehmen, die Beschäftigung aufbauen, unter dem Schwellenwert mit denen darüber. Die beiden Autoren können die aufgestellte These eines geringeren Anteils für die kleineren Betriebe nicht untermauern. Wagner et al. (2001b) verwenden in ihrer Studie ökonometrische Methoden ebenfalls auf Basis des IABBetriebspanels. Auch sie können den vermuteten Schwellenwerteffekt nicht nachweisen. Beide Arbeiten verzichten allerdings darauf, die Tatsache, ob ein Unternehmen unter das Gesetz fällt, als endogene Entscheidung zu modellieren und setzen ihre Ergebnisse somit der Gefahr der Verzerrung durch Selbstselektion aus. Ein etwas abweichendes Ziel verfolgen Boockmann und Hagen (2001): Sie untersuchen Bestimmungsgründe für die Einstellung von Mitarbeitern in befristeten Verträgen. Dabei vergleichen sie den Einfluss der Betriebsgröße auf eine binäre Outcome-Variable, die darüber Auskunft gibt, ob ein Betrieb auf das Instrument befristeter Arbeitsverträge zurückgreift. In einer Regression auf diese Dummy-Variable finden sie, dass eine Betriebsgrößen-Dummy-Variable für sechs bis zehn Beschäftigte nach der 1996er Gesetzesänderung signifikant geringer ist als vor der Novellierung und interpretieren dies als das Ergebnis dieser Maßnahme. Um die Auswirkungen von Schwellenwerten im deutschen Kündigungsschutzrecht zu untersuchen, verwendet Verick (2004) einen Diff-in-Diff-Schätzer mit all seinen Problemen der Modelspezifizierung und der funktionalen Form. Er findet nur schwache Indizien für den erwarteten Effekt und verweist auf die Möglichkeit unvollständiger Information seitens der betrieblichen Entscheidungsträger über die Details des KSchG, insbesondere die Berechnung der Zahl der Beschäftigten. Abhängige Variablen der Untersuchung von Bauer et al. (2004) sind Einstellungen und Entlassungen von Betrieben. Auch Ihnen gelingt es trotz eines großen Datensatzes nicht, signifikante Einflüsse des KSchG in ihrer Schätzung nachzuweisen. 


\section{B 1.2 Kündigungsschutz für Ältere}

Sowohl in der im BGB verankerten Regelung zur Kündigung als auch im KSchG selbst werden Ältere besonders behandelt. Zwar enthält das BGB keinen expliziten Verweis auf das Alter der Beschäftigten, macht aber Kündigungsfristen abhängig von der Dauer der Betriebszugehörigkeit ab dem 25. Lebensjahr. Diese steigen von einem Monat für mindestens zwei Jahre Beschäftigte auf bis zu sieben Monate für Personen, die dem Betrieb mindestens 20 Jahre angehörten. Implizit sind damit ältere Beschäftigte - und nur solche weisen lange Erwerbsbiografien im Betrieb auf - schon von der BGB-Regelung begünstigt. Allerdings rückt diese Klausel der Neueinstellung von Älteren keine weiteren Steine in den Weg und kann daher nicht für die geringen Wiedereinstellungschancen Älterer als Erklärung herangezogen werden.

Anders dagegen das KSchG: Im Falle betriebsbedingter Kündigung muss der Arbeitgeber in der Sozialauswahl auf die Entlassung langjährig Beschäftigter Älterer und behinderter Arbeitnehmer, sowie solcher mit Unterhaltspflichten zunächst verzichten. Auch muss der Arbeitgeber dem Arbeitnehmer auf Verlangen die Gründe angeben, die zur Auswahl der Entlassenen geführt haben. Der Arbeitnehmer hat dann die Tatsachen zu beweisen, die die Kündigung als sozial ungerechtfertigt erscheinen lassen und diese damit rechtsunwirksam machen.

Zwar ist die Sozialauswahl in der jüngsten Novellierung des Gesetzes mit Wirkung zum 1. 1. 2004 gelockert worden. Nach aktuellem Gesetzesstand in $\S 1$ Absatz 3 Satz 2 KSchG kann der Arbeitgeber bei der betriebsbedingten Kündigung von der Regel der sozialen Auswahl abweichen und Arbeitnehmer nicht einzubeziehen, „,eren Weiterbeschäftigung, insbesondere wegen ihrer Kenntnisse, Fähigkeiten und Leistungen oder zur Sicherung einer ausgewogenen Personalstruktur des Betriebes, im berechtigten betrieblichen Interesse liegt.“ Für den Untersuchungszeitraum allerdings waren die einschränkenden Bemerkungen noch nicht eingeführt, sodass vermutet werden kann, dass vom KSchG ein abschreckender Effekt auf einstellungswillige Unternehmer ausgeht.

Ein weiterer, zwar nicht gesetzlich festgelegter, aber wohl bedeutsamer Schutzmechanismus für Ältere ist ein in vielen Tarifverträgen und Betriebsvereinbarungen beschlossenes Kündigungsverbot für Beschäftigte, die eine gewisse Altersgrenze überschritten haben. Je nach Branche und Region sind in einer Vielzahl von Vereinbarungen Altersgrenzen von 50, 53 oder 55 Jahren - meist gekoppelt an eine Mindestdauer der Betriebszugehörigkeit - festgelegt, von denen an keine ordentlichen Kündigungen mehr ausgesprochen werden dürfen. ${ }^{63}$

Besonderes Ziel dieses Kapitels ist es, die Frage nach einer möglichen beschäftigungshemmenden Effekt der Schwellenwerte auf die Gruppe der Älteren auszuweiten, da sie - wie hier ausgeführt - besonderen, strengeren Vorschriften unterliegen. Eine empirische Untersuchung der vermuteten Wirkung liegt bisher nicht vor.

\footnotetext{
${ }^{63}$ Einen Eindruck von der Vielfalt der Regelungen erhält man bei Bispinck (2002) S. 19ff.
} 


\section{B 2 Datengrundlage Kündigungsschutz: Linked Employer Employee Datensatz}

Für die Zwecke der Untersuchung sind sowohl individuelle Daten des einstellenden Betriebes als auch die Altersinformation auf Personenebene notwendig. Auf betrieblicher Seite eignet sich dazu das oben vorgestellte Betriebspanel des IAB. Hier entspricht die verwendete Betriebsdefinition als produktionstechnische Einheit der des KSchG. Außerdem lassen die Angaben im IAB-Betriebspanel eine Differenzierung der Belegschaften in mithelfende Familienangehörige, Auszubildende und Teilzeitbeschäftigte zu, die für die Berechnung der relevanten Betriebsgröße wichtig ist.

Die verwendeten Beobachtungen beschränken sich auf diejenigen Betriebe, die mindestens in einem Jahr eine positive Geschäftsentwicklung erwarteten, nie aber eine negative. Die Stichprobe verringert sich damit auf 1883 Betriebe für die Schätzungen bis 1997, bis 2003 verringert sich der Datensatz weiter auf 697 Fälle. Die Grundgesamtheit des IAB-Betriebspanels bilden Betriebe mit mindestens einem sozialversicherungspflichtig Beschäftigten. Die einzelnen Panelwellen enthalten aufgeschlüsselte Informationen über die Anzahl der Beschäftigten jeweils zum Stichtag 30. Juni des betreffenden Jahres.

Probleme bereitet die Erfassung von Teilzeitarbeit im IAB-Panel, die zu Ad-hoc-Annahmen bei der Analyse führte. Die Angaben im Panel über Anteile der Belegschaft, die wöchentlich bis zu 15 oder 24 Stunden oder Vollzeit arbeiten, entspricht nicht genau der Unterscheidung, die das Gesetz bezüglich des Ausmaßes von Teilzeitarbeit vorsieht. Man muss daher mit Fehlzuordnungen von Betrieben mit Teilzeitbeschäftigung in die Treatment- und Kontrollgruppe rechnen.

Zeitlich befristete Arbeit ist für die Belange dieser Studie nur unzureichend erfasst. Informationen über die Dauer der Befristung, wie sie eine richtige Anrechnung nach der KSchGRegelung verlangen würde, sind im Datensatz leider nicht enthalten. Dies ist problematisch, da ausschließlich Betriebe, die aufgrund ihrer geringen Beschäftigtenzahl der Kontrollgruppe zugeschlagen werden müssten, in die Treatmentgruppe geraten. Dies mag die erwarteten Effekte verringern. Ein weiteres Problem könnte die Möglichkeit nichtzufälliger Panelmortalität darstellen. Um Verzerrungen in den Ergebnissen der nachfolgenden empirischen Auswertung auszuschließen, wurde eine mögliche Korrelation mit dem Treatmentstatus untersucht und kein Anhaltspunkt dafür gefunden. ${ }^{64}$

Trotz der angeführten Defizite erfüllen die Daten des IAB- Betriebspanels die wesentlichen Auswahlbedingungen. Ein weiterer Grund für die Verwendung der Daten ist Verknüpfbarkeit mit den persönlichen Individualinformationen der Sozialversicherungsträger. Sie ist notwen-

\footnotetext{
${ }^{64}$ Um zu untersuchen, ob der Treatmentstatus als Prädiktor für Panelmortalität in den darauf folgenden Jahren dient, wurden einfache Probit-Schätzungen dieses Treatmentstatus auf einen Dummy regressiert, der angab, ob der Betrieb aus dem IAB-Panel ausgeschieden ist. In den meisten Fällen konnte kein signifikanter Einfluss festgestellt werden.
} 
dig, um das Alter der Neueingestellten einer Periode zu erfassen. Der verknüpfte LinkedEmployer-Employee Datensatz (LIAB) bildet daher die Datengrundlage für die folgenden Auswertungen.

\section{B 3 Mikroökonometrischer Ansatz: Das Regression Dis- continuity Design}

Ein für diese Fragestellung angemessener Evaluationsansatz ist ein RDD-Ansatz in seiner scharfen Form. ${ }^{65}$ Er findet dann Anwendung, wenn die Programmteilnahme (hier: dem KSchG unterliegen) deterministisch von einer Selektionsvariablen $z_{i}$ abhängt: Sobald diese einen Schwellenwert $\mathrm{z}_{0}$ übersteigt, nimmt die Indikatorvariable $\mathrm{x}_{\mathrm{i}}$ den Wert 1 an, sonst 0 . Die beobachtete Outcomevariable $\mathrm{y}_{\mathrm{i}}$ kann dann beschrieben werden als

$$
\mathrm{y}_{\mathrm{i}}=\mathrm{y}_{0 \mathrm{i}}+\mathrm{x}_{\mathrm{i}}\left(\mathrm{z}_{\mathrm{i}}\right) \beta_{\mathrm{i}}
$$

mit $y_{0 \mathrm{i}}$ als Outcomevariable im möglicherweise hypothetischen Fall, dass Betrieb i der Kontrollgruppe angehört. $\beta_{\mathrm{i}}$ steht für den Treatmenteffekt, der in diesem Fall darin besteht, nicht vor der Schwelle des KSchG zu stehen und damit nicht im Beschäftigungswachstum gebremst zu sein.

Gegenüber anderen Evaluationsmethoden besteht der Vorteil dieses Ansatzes darin, dass keine Annahmen über die Modellspezifikation oder über die Parametrisierung getroffen werden müssen. Nach Hahn et al. (2001, S. 16) liegt dies daran, dass die Effekte aller nicht berücksichtigten Variablen im Schwellenwert stetig sind und daher in $\mathrm{y}_{0 \mathrm{i}}$ eingehen. Solange dies der Fall ist, hebt sich ihr Effekt in der Differenz zwischen Kontroll- und Treatmentgruppe auf.

Battistin und Rettore (2003) heben hervor, dass die scharfe Form des RDD zwei erhebliche Nachteile aufweist: Erstens ist die Anwendbarkeit der Methode beschränkt auf solche Fälle, in denen die Auswahl von Treatment- und Kontrollgruppe ausschließlich von prädeterminierten Variablen abhängt. Dies für das deutsche Kündigungsschutzrecht allerdings der Fall: Für einen gegebenen Wert von z (Anzahl der Beschäftigten im Betrieb nach Definition des $\mathrm{KSchG}$ ) ist die Zuordnung zu einer der Gruppen eindeutig. Verwendet man die Beschäftigtenzahlen vor der Änderung des Schwellenwertes von fünf auf zehn Mitarbeiter, kann die Kovariable $\mathrm{z}$ als prädeterminiert angesehen werden.

Battistin und Rettore weisen auf einen zweiten Nachteil der Methode hin: Die Schätzung der Programmeffekte im RDD erlaubt nur die Identifizierung von lokalen Effekten der Intervention am Schwellenwert. Daher muss man sich bei der Interpretation der Ergebnisse der Tatsache bewusst sein, dass Effekte nur für diese Subgruppe der Betriebe nahe am Schwellenwert

\footnotetext{
${ }^{65}$ Obwohl mit den verfügbaren Daten auch ein Matching-Ansatz denkbar wäre, verbietet das Common-SupportProblem eine solche Art der Schätzung, vgl. Lechner (2001). Neuere Anwendungen von RDD-Schätzungen sind Hahn et al. (1999), Carling und Larsson (2002) oder Buddelmeyer und Skoufias (2003).
} 
ermittelt werden können. Andererseits entspricht die so gewonnene Aussage der untersuchten Fragestellung, die die Kleinbetriebe und ihre Beschäftigungsdynamik im Blickpunkt hat.

Beide erwähnten Nachteile stellen daher die Anwendbarkeit der Methode auf den Gegenstand dieser Untersuchung nicht in Frage.

\section{B 3.1 Identifikation von Treatment-Effekten}

Welche Annahmen müssen getroffen werden, um Treatment-Effekte zu messen? Hahn et al. (2001) zeigen, dass Treatment-Effekte unter schwachen Annahmen identifiziert und nichtparametrisch geschätzt werden können.

Neben dem RDD-Ansatz, der verlangt, dass die Indexvariable $x_{i}$ eine diskontinuierliche Funktion von $z_{i}$ sein muss, reicht die Annahme aus, dass die Outcomevariable $y_{0 i}$ stetig in $z$ am Punkt $\mathrm{z}_{0}$ ist. Interpretiert man den Ausdruck $\mathrm{E}\left(\mathrm{y}_{0 \mathrm{i}} \mid \mathrm{z}_{\mathrm{i}}=\mathrm{z}\right)$ als Funktion von $\mathrm{z}$, ist zur Identifikation von Treatment-Effekten nur Stetigkeit an der Stelle $\mathrm{z}_{0}$ notwendig. Das bedeutet, dass Betriebe nahe dem Schwellenwert - angenommen sie unterlägen nicht dem KSchG - ein ähnliches Einstellungsverhalten aufweisen, unabhängig davon, ob sie über oder unter dem Schwellenwert liegen. Die zentrale identifizierende Annahme scheint plausibel, da der Schwellenwert $z_{0}$ für die Betriebe zufällig gewählt wurde. Die untersuchte Gesetzesänderung stellt mit seiner Festsetzung eines willkürlichen neuen Schwellenwertes ein natürliches Experiment dar. Daraus ergibt sich eine komfortable Situation für die Kontrolle der Selbstselektion der Betriebe in das Treatment. Die identifizierende Annahme sieht vor, dass ein solches Verhalten der Betriebe zum Zeitpunkt des Festlegens von Treatment- und Kontrollgruppe noch nicht eingesetzt hat. Zudem kann man Probleme mit Drop-outs aus dem Treatment ausschließen. Kein Betrieb, der den Schwellenwert von zehn Beschäftigten überschreitet, kann sich der Gefahr entziehen, nach der Kündigung eines Mitarbeiters mit hohen Forderungen konfrontiert zu werden. Weder Ein- noch Austritt aus dem Treatment sind von den Beobachtungseinheiten beeinflussbar.

Dennoch müssen einige einschränkende Bemerkungen angefügt werden: Bei der Schätzung von Treatment-Effekten für Großprogramme wie dem Kündigungsschutzrecht treten Probleme mit der Stable Unit Value Assumption auf. Diese sieht vor, dass die untersuchte Maßnahme keinen Einfluss auf das Verhalten der Beobachtungen in der Kontrollgruppe ausübt. Für die vorliegende Untersuchung könnte diese Annahme verletzt sein. Mögliche negative Effekte auf die Arbeitsnachfrage der kleineren Betriebe könnte Unternehmen oberhalb des Schwellenwertes die Suche nach Mitarbeitern erleichtern.

Zudem könnte neben dem Einstellungsverhalten von Kleinbetrieben auch das der über fünf bzw. zehn Beschäftigten von den Schwellenwerten beeinflusst sein. Es wird angenommen, dass Betriebe, die sich einer verstärkten Nachfrage ausgesetzt sehen, sich im Einstellungsverhalten nicht von den Schwellenwerten leiten lassen. Die verwendete Stichprobe beschränkt sich daher auf solche Betriebe, die für mindestens ein Jahr positive Geschäftserwartungen angaben. Die Geschäftserwartungen dienen als verlässlicherer Indikator der Einstellungsab- 
sicht weiterer Beschäftigter als die tatsächliche, aktuelle Geschäftsentwicklung, da sie einen zukünftigen Bedarf an Mitarbeitern widerspiegelt, der für die aktuelle Einstellungsentscheidung von ursächlicher Bedeutung ist.

Auch kann ein Anlass möglicher Selbstselektion für die Untersuchung der zweiten Änderung des KSchG nicht ausgeschlossen werden. So könnte die Rücknahme der Gesetzesänderung durch die Regierung Schröder im Jahr 1998 von betrieblichen Entscheidungsträgern schon vor dem Erhebungszeitpunkt im Juni antizipiert worden sein. Die Wiederausweitung des Gesetzes war nämlich ein zentrales Wahlversprechen der damaligen Opposition. Allerdings bezieht sich die Zuordnung zu Treatment- und Kontrollgruppe auf die Beschäftigtenzahl Ende Juni 1998, wenn das IAB-Betriebspanel erhoben wird. Selbstselektion wäre hier nur dann ein Problem, wenn Arbeitgeber schon im Frühjahr 1998 in Erwartung eines Sieges der damaligen Opposition eine entsprechende Anpassung der Beschäftigtenzahl vorgenommen hätte.

Die deutsche Arbeits- und Sozialgesetzgebung sieht zudem neben den Schwellenwerten des Kündigungsschutzrechtes weitere betriebsgrößenabhängige Geltungsbereiche von Gesetzen vor. Im Januar 2001 hatten Mitarbeiter von Betrieben mit mehr als fünf Beschäftigten das Recht, einen Betriebsrat zu wählen. Betriebe ab dieser Größe mussten getrennte Toiletten für Männer und Frauen bereitstellen. Weitere Regelungen treten in Kraft, sobald die Schwellen von zehn und zwanzig Mitarbeitern überschritten werden (vgl. Wagner et al. (2001b)). Dies kann zu einer gewissen Selbstselektion an der Schwelle geführt haben.

Der RDD-Schätzer kann als Spezialfall einer „Selection on observables“ betrachtet werden, da aus dem Selektionsmechanismus und der oben getroffenen Annahme folgt, dass also die Outcomevariable lokal unabhängig von der Teilnahmevariable ist

$$
\left(\mathrm{y}_{1}, \mathrm{y}_{0}\right) \perp \mathrm{x} \mid \mathrm{z}=\mathrm{z}_{0} .
$$

Hahn et al. (2001) zeigen, wie sich die Effekte schätzen lassen. Unabhängig davon, ob man einheitliche Effekte für alle Betriebe unterstellt oder ob man unterschiedliche Effekte für die einzelnen Unternehmen zulässt. Im ersten Fall identischer Effekte gilt: $\beta_{i}=\beta$ für alle i. Der local average treatment effect (LATE) $\beta$ des Umstandes, den Schwellenwert überschritten zu haben, an der Stelle $\mathrm{z}_{0}$ kann dann identifiziert werden als

$$
\beta=y^{+}-y^{-}
$$

mit $\mathrm{y}^{+} \equiv \lim _{z \rightarrow z_{0}^{+}} \mathrm{E}\left[\mathrm{y}_{\mathrm{i}} \mid \mathrm{z}_{\mathrm{i}}=\mathrm{z}\right]$ und $\mathrm{y}^{-} \equiv \lim _{z \rightarrow z_{0}^{-}} \mathrm{E}\left[\mathrm{y}_{\mathrm{i}} \mid \mathrm{z}_{\mathrm{i}}=\mathrm{z}\right]$ als Grenzwerten der erwarteten Outcome-Variablen, wenn man sich der Schwelle von oben bzw. von unten nähert.

Im Falle heterogener Effekte für die Betriebe lässt sich ein nach Hahn et al. (2001) erwarteter Treatment-Effekt als

$$
\mathrm{E}\left(\beta_{\mathrm{i}} \mid \mathrm{z}_{\mathrm{i}}=\mathrm{z}_{0}\right)=\mathrm{y}^{+}-\mathrm{y}^{-}
$$


identifizieren. Dazu muss neben den Annahmen, die für die Identifikation einheitlicher Effekte notwendig waren, auch eine lokale Stetigkeitsannahme bezüglich der erwarteten Effekte eingeführt werden: Am Schwellenwert $z=z_{0}$ muss $E\left(\beta_{i} \mid z_{i}=z\right)$ eine stetige Funktion von $z$ sein. Wieder spricht der quasi-experimentelle Charakter der untersuchten Situation für diese Annahme. Darüber hinaus muss eine bedingte Unabhängigkeit der Treatment-Variablen $x_{i}$ und des lokalen individuellen Treatment-Effektes $\beta_{\mathrm{i}}$ an der Stelle $\mathrm{z}_{0}$ getroffen werden: Für Unternehmen nahe am Schwellenwert von zehn Beschäftigten sollen Umfang des TreatmentEffektes und Treatmentstatus unabhängig voneinander sein. Wieder garantiert die zufällige Einordnung der Unternehmen in Treatment- und Kontrollgruppe, dass die Annahme gerechtfertigt erscheint. Man kann davon ausgehen, dass eine Selbstselektion der Unternehmen in eine der Gruppen je nach der erwarteten Beeinträchtigung durch die Maßnahme nur in geringem Maße möglich war.

Sowohl im Fall fixer, als auch variabler Effekte, kann der LATE unter Zuhilfenahme einer Reihe begründbarer Annahmen identifiziert werden.

\section{B 3.2 Schätzung der Treatment-Effekte}

In beiden Fällen, bei Annahme einheitlicher Effekte und bei Zulassen individueller Effekte, kann der LATE im scharfen Fall des RDD konsistent geschätzt werden als

$$
\hat{\beta}=\hat{y}^{+}-\hat{y}^{-}
$$

wobei $\hat{y}^{+}$und $\hat{y}^{-}$konsistente Schätzer der Grenzwerte $\mathrm{y}^{+}$und $\mathrm{y}^{-}$darstellen. Zur Schätzung der beiden Erwartungswerte sind verschiedene Vorgehensweisen vorgeschlagen worden. Da einfache, einseitige Kerndichteschätzer unerwünschte statistische Eigenschaften an den Randstellen des Trägers aufweisen, empfehlen Hahn et al. (2001) und Porter (2003), lokale parametrische Restriktionen in der angenommenen Beziehung zwischen Outcomevariable y und Selektionsvariable z zu unterstellen. Wie auch Fan und Gijbels (1996, S. 60ff.) hervorheben, lässt sich das so genannte Boundary-Problem mit Hilfe von lokal linearen Regressionsschätzungen (LLR) umgehen. Daher schlagen Hahn et al. (1999 und 2001) eine lokale lineare Regression vor, die auch hier mit der Spezifikation eines Epanechnikov Kerndichteschätzers verwendet wurde. Um nicht anderen Schwellenwerten im Arbeitsrecht in die Quere zu kommen und so die Schätzung um die Einflüsse ihrer Wirkung zu verfälschen, wurden hier Bandbreiten zwischen 1 und 5 für die Schätzung der Werte $\mathrm{y}^{+}$und $\mathrm{y}^{-}$gewählt. Die asymptotischen Eigenschaften des Schätzers $\hat{\beta}_{\mathrm{LLR}}$ sind abgeleitet von Hahn et al. (1999, S. 11ff).

\section{B 4 Mikroökonometrische Ergebnisse}

Für die Schätzung der Treatment-Effekte wurde der vor kurzem in Stata 8.2 installierte Befehl für lokale polynomiale Regressionen verwendet. Die Varianzen sind per Bootstrap mit 1000 Ziehungen aus den Originaldaten geschätzt worden. Dabei werden zunächst die Ergebnisse 
für eine allgemeine Schätzung der Wirkung des Schwellenwertes im KSchG dargestellt, danach folgen die Resultate für die Effekte auf die Einstellungsaussichten von Älteren.

\section{B 4.1 Altersunabhängige Ergebnisse}

Tab. 4 stellt die geschätzten Treatment-Effekte auf das Beschäftigungswachstum nach der ersten Gesetzesänderung dar. Im ersten Teil sind die Auswirkungen auf das Wachstum von Juni 1996 bis Juni 1997, im zweiten Teil je bis zum Juni 1998 zu sehen.

Tab. 4: LATE Schätzungen des Beschäftigungswachstums; Outcome-Variable: Beschäftigungswachstum von 6/96 bis 6/97 und von 6/96 bis 6/98 (geschätzte Std. abw. in Klammern)

\begin{tabular}{|c|c|c|c|c|c|c|c|c|}
\hline \multirow[b]{2}{*}{ Bandbreite } & \multicolumn{4}{|c|}{$\begin{array}{c}\text { Beschäftigungswachstum nach KSchG } \\
\text { zwischen } 6 / 96 \text { und 6/97 }\end{array}$} & \multicolumn{4}{|c|}{$\begin{array}{c}\text { Beschäftigungswachstum nach KSchG } \\
\text { zwischen } 6 / 96 \text { und 6/98 }\end{array}$} \\
\hline & $\hat{\mathrm{y}}^{-}$ & $\hat{y}^{+}$ & LATE & $\mathrm{n}$ & $\hat{\mathrm{y}}^{-}$ & $\hat{\mathrm{y}}^{+}$ & LATE & $\mathrm{n}$ \\
\hline 1 & 0,669 & 1,213 & $\begin{array}{c}0,544 \\
(2,112)\end{array}$ & 38 & $-0,143$ & 1,272 & $\begin{array}{c}1,415 \\
(3,421)\end{array}$ & 26 \\
\hline 2 & 0,777 & 1,765 & $\begin{array}{c}0,988 \\
(1,587)\end{array}$ & 84 & 0,134 & 0,690 & $\begin{array}{c}0,556 \\
(2,317)\end{array}$ & 58 \\
\hline 3 & 0,744 & 1,333 & $\begin{array}{c}0,589 \\
(1,271)\end{array}$ & 124 & $-0,052$ & 0,725 & $\begin{array}{c}0,777 \\
(1,784)\end{array}$ & 85 \\
\hline 4 & 0,663 & 1,113 & $\begin{array}{c}0,450 \\
(1,086)\end{array}$ & 165 & 0,128 & 0,424 & $\begin{array}{c}0,296 \\
(1,481)\end{array}$ & 112 \\
\hline 5 & 0,523 & 0,619 & $\begin{array}{c}0,096 \\
(0,926)\end{array}$ & 195 & 0,256 & 0,292 & $\begin{array}{c}0,036 \\
(1,335)\end{array}$ & 138 \\
\hline
\end{tabular}

Quelle: IAB-Betriebspanel, eigene Berechnungen, ${ }^{* * *} 1 \%,{ }^{* *} 5 \%,{ }^{*} 10 \%$ Signifikanzniveau

Es gibt keinen Hinweis darauf, dass der 1996 neu eingeführte Schwellenwert von zehn Beschäftigten Auswirkungen auf das Einstellungsverhalten der kleinen Betriebe hat. Obwohl alle LATE-Ergebnisse für das erste Jahr das erwartete positive Vorzeichen aufweisen, ist keines davon signifikant. Dies gilt unabhängig von der Wahl der Bandbreite. Die Ergebnisse sind in Anhang B graphisch dargestellt. Schätzungen von erwartetem Beschäftigungswachstum für Betriebe sind dabei abgetragen über die Anzahl der sozialversicherungspflichtig Beschäftigten. Der Sprung an der Stelle von fünf Mitarbeitern stellt die Schätzung des lokalen Treatment-Effektes dar.

Diese Ergebnisse bestätigen den Befund aus den Studien von Friedrich und Hägele (1997), Wagner et al. (2001b), Verick (2004) und Bauer et al. (2004). Keine findet eine empirische Bestätigung der Thesen, die sowohl in der ökonomischen Theorie als auch in der öffentlichen Debatte vorgebracht werden.

Einschränkend muss allerdings erwähnt werden, dass der Schwellenwert erst im Herbst 1999 seine Wirkung voll hätte entfalten können, wenn die Übergangsphase für die Bestandmitarbeiter ausgelaufen wäre. Dennoch besteht Anlass zur Vermutung, dass ein Anpassungsprozess an die nach 1996 neue Gesetzeslage schon im Beobachtungszeitraum hätte beginnen müssen, da die Entlassung von Beschäftigten ein langwieriger Prozess sein kann. Zudem hatte der Schwellenwert auch vor Auslaufen der Übergangsphase ökonomische Bedeutung für all die Betriebe, die in der Zwischenzeit Einstellungen vorgenommen hatten. 
Tab. 5 und Tab. 6 stellen die geschätzten Treatment-Effekte für das Wachstum zwischen Juni 1998 und den Monaten Juni 1999 bis 2003 bei dem dann geltenden Schwellenwert von zehn Beschäftigten.

Im Großen und Ganzen bleiben die Ergebnisse auch nach der zweiten Gesetzesänderung insignifikant. Für das Wachstum bis 2002 und 2003 konnten wieder unabhängig von der gewählten Bandbreite keine Hinweise darauf gefunden werden, dass sich Kleinbetriebe aufgrund des Schwellenwertes mit ihren Einstellungen zurückhielten. Für die Jahre 1999 und 2001 dagegen finden sich Ausnahmen bei LLR-Schätzungen mit kleinen Bandbreiten. Bei einer Bandbreite von 1 lässt sich für die Zeitspanne zwischen Mitte 1998 und Mitte 1999 ein signifikant höherer Wert für die Treatmentgruppe mit ihren Betrieben über dem Schwellenwert beobachten als für die Kontrollgruppe. Im Gegensatz dazu ergeben die Schätzungen mit den Bandbreiten 1 und 2 für die Zeitspanne bis Juni 2001 sogar einen schwach signifikant negativen Parameter.

Tab. 5: LATE Schätzungen des Beschäftigungswachstums; Outcome-Variable: Beschäftigungswachstum von $6 / 98$ bis 6/99 und von 6/98 bis 6/01 (geschätzte Std. abw. in Klammern)

\begin{tabular}{|c|c|c|c|c|c|c|c|c|}
\hline \multirow[b]{2}{*}{ Bandbreite } & \multicolumn{4}{|c|}{$\begin{array}{c}\text { Beschäftigungswachstum nach KSchG } \\
\text { zwischen } 6 / 98 \text { und } 6 / 99\end{array}$} & \multicolumn{4}{|c|}{$\begin{array}{c}\text { Beschäftigungswachstum nach KSchG } \\
\text { zwischen } 6 / 98 \text { und } 6 / 01\end{array}$} \\
\hline & $\hat{\mathrm{y}}^{-}$ & $\hat{\mathrm{y}}^{+}$ & LATE & $\mathrm{n}$ & $\hat{\mathrm{y}}^{-}$ & $\hat{\mathrm{y}}^{+}$ & LATE & $\mathrm{n}$ \\
\hline 1 & 0,413 & 0,831 & $\begin{array}{c}0,418 \\
(0,498)\end{array}$ & 90 & 1,096 & 0,600 & $\begin{array}{l}-0,496 \\
(0,969)\end{array}$ & 65 \\
\hline 2 & 0,308 & 0,532 & $\begin{array}{c}0,224 \\
(0,400)\end{array}$ & 184 & 1,081 & 0,494 & $\begin{array}{l}-0,587 \\
(0,681)\end{array}$ & 129 \\
\hline 3 & 0,272 & 0,586 & $\begin{array}{c}0,313 \\
(0,363)\end{array}$ & 283 & 1,026 & 0,713 & $\begin{array}{l}-0,313 \\
(0,610)\end{array}$ & 208 \\
\hline 4 & 0,265 & 0,422 & $\begin{array}{c}0,157 \\
(0,324)\end{array}$ & 399 & 1,013 & 0,672 & $\begin{array}{l}-0,341 \\
(0,591)\end{array}$ & 301 \\
\hline 5 & 0,262 & 0,461 & $\begin{array}{c}0,199 \\
(0,328)\end{array}$ & 521 & 1,008 & 0,344 & $\begin{array}{l}-0,663 \\
(0,615)\end{array}$ & 393 \\
\hline
\end{tabular}

Quelle: IAB-Betriebspanel, eigene Berechnungen, ${ }^{* * *} 1 \%,{ }^{* *} 5 \%,{ }^{*} 10 \%$ Signifikanzniveau

Tab. 6: LATE Schätzungen des Beschäftigungswachstums; Outcome-Variable: Beschäftigungswachstum von $6 / 98$ bis $6 / 02$ und von $6 / 98$ bis $6 / 03$ (geschätzte Std. abw. in Klammern)

\begin{tabular}{|c|c|c|c|c|c|c|c|c|}
\hline \multirow[b]{2}{*}{ Bandbreite } & \multicolumn{4}{|c|}{$\begin{array}{c}\text { Beschäftigungswachstum nach KSchG } \\
\text { zwischen } 6 / 98 \text { und } 6 / 02\end{array}$} & \multicolumn{4}{|c|}{$\begin{array}{c}\text { Beschäftigungswachstum nach KSchG } \\
\text { zwischen } 6 / 98 \text { und } 6 / 03\end{array}$} \\
\hline & $\hat{y}^{-}$ & $\hat{y}^{+}$ & LATE & $\mathrm{n}$ & $\hat{\mathrm{y}}^{-}$ & $\hat{y}^{+}$ & LATE & $\mathrm{N}$ \\
\hline 1 & 1,318 & 1,612 & $\begin{array}{c}0,295 \\
(1,255)\end{array}$ & 52 & 1,565 & 1,339 & $\begin{array}{l}-0,225 \\
(1,737)\end{array}$ & 35 \\
\hline 2 & 1,255 & 1,226 & $\begin{array}{l}-0,029 \\
(1,024)\end{array}$ & 106 & 1,856 & 1,286 & $\begin{array}{l}-0,570 \\
(1,367)\end{array}$ & 72 \\
\hline 3 & 1,206 & 1,279 & $\begin{array}{c}0,073 \\
(0,852)\end{array}$ & 162 & 1,735 & 0,194 & $\begin{array}{l}-1,541 \\
(1,211)\end{array}$ & 111 \\
\hline 4 & 1,195 & 0,963 & $\begin{array}{l}-0,231 \\
(0,784)\end{array}$ & 232 & 1,708 & $-0,463$ & $\begin{array}{l}-2,171^{\star *} \\
(1,164)\end{array}$ & 158 \\
\hline 5 & 1,190 & 0,402 & $\begin{array}{l}-0,788 \\
(0,831)\end{array}$ & 310 & 1,696 & $-0,296$ & $\begin{array}{l}-1,993^{* *} \\
(1,068)\end{array}$ & 223 \\
\hline
\end{tabular}

Quelle: IAB-Betriebspanel, eigene Berechnungen, ${ }^{* * *} 1 \%,{ }^{* *} 5 \%,{ }^{*} 10 \%$ Signifikanzniveau 
Dies deutet gar auf ein geringeres Beschäftigtenwachstum der Betriebe hin, die bereits vor der Gesetzesänderung mehr als zehn Angestellte im Sinne des KSchG aufwiesen. Diese Teilergebnisse widersprechen allen theoretischen Überlegungen. Im Hinblick auf die unter $n$ angegeben Fallzahlen, auf die für die Schätzung zurückgegriffen werden konnte, erscheint es allerdings zweifelhaft, ob die Ergebnisse generalisiert werden können. Aufgrund des geringen Stichprobenumfanges mögen Signifikanzaussagen auch von Ausreißern bestimmt sein.

In der Gesamtansicht erweisen sich die Ergebnisse als weitgehend robust gegenüber der Wahl der Bandbreite und des Untersuchungszeitraumes. Die Hypothese, dass Beschäftigungsaufbau in Kleinbetrieben von Schwellenwerten des Kündigungsschutzgesetzes behindert wird, ließ sich nicht bestätigen.

\section{B 4.2 Ergebnisse für Ältere}

In Tab. 15 finden sich die geschätzten Treatment-Effekte auf die Einstellung neuer Mitarbeiter von mindestens 50 Jahren $^{66}$ nach der ersten Gesetzesänderung. Dabei sind in der linken Hälfte die Schätzungen der Effekte auf die Einstellungen von Älteren von Juni 1996 bis Juni 1997, im zweiten Teil diejenigen bis zum Juni 1998 zu sehen.

Wieder gelingt es trotz des geänderten Auswertungsziels und der Berücksichtigung nur der Einstellungen der besonders geschützten Älteren nicht, signifikante Unterschiede zwischen den beiden Schätzwerten nachzuweisen. Der oben ausgeführten Argumentation folgend hätte der 1996 auf zehn Beschäftigte erhöhte Schwellenwert einen negativen Effekt auf das Einstellungsverhalten kleiner Betriebe haben müssen. Dennoch weisen nahezu alle Schätzungen für die erste Änderung sogar negative Vorzeichen auf und zeigen damit an, dass Betriebe oberhalb der Schwelle sogar weniger Ältere neu einstellen als Kleinbetriebe, die nun erst recht keinen Anreiz erst haben müssten Ältere einzustellen. Statistisch signifikant ist dieses negative Vorzeichen jedoch nur im Falle einer Bandbreite von eins. Diese Resultate bestätigen und erweitern die Ergebnisse aus den Studien von Friedrich und Hägele (1997), Wagner et al. (2001b), Verick (2004) und Bauer et al. (2004), die generell keinen empirischen Nachweis für die beschäftigungshemmende Wirkung der Schwellenwerte im KSchG vorlegen konnten. Selbst für die wie oben beschrieben besonders geschützte Gruppe der Älteren konnte ein solcher Nachweis nicht erbracht werden.

Genauso wie in der allgemeinen Untersuchung muss man einräumen, dass der Schwellenwert erst nach der Übergangsfrist voll zur Geltung gekommen wäre. An den theoretischen Überlegungen ändert sich - wie oben erwähnt - allerdings nichts.

\footnotetext{
${ }^{66}$ Für alternative Definitionen der Älteren mit Grenzen von 45 und 55 Jahren ergaben sich sehr ähnliche Ergebnisse. Negative Werte der Grenzwertschätzungen erklären sich dadurch, dass sie mittels lokaler, linearer Regression ermittelt wurden.
} 
Tab. 7: $\quad$ LATE Schätzungen der Anzahl der Einstellungen von 50-Jährigen und Älteren von 6/96 bis 6/97 und von 6/96 bis 6/98 (geschätzte Std. abw. in Klammern)

\begin{tabular}{|c|c|c|c|c|c|c|c|c|}
\hline \multirow[b]{2}{*}{ Bandbreite } & \multicolumn{4}{|c|}{$\begin{array}{c}\text { Einstellungen } \\
\text { zwischen } 6 / 96 \text { und 6/97 }\end{array}$} & \multicolumn{4}{|c|}{$\begin{array}{c}\text { Einstellungen } \\
\text { zwischen } 6 / 96 \text { und 6/98 }\end{array}$} \\
\hline & $\hat{\mathrm{y}}^{-}$ & $\hat{\mathrm{y}}^{+}$ & LATE & $\mathrm{n}$ & $\hat{\mathrm{y}}^{-}$ & $\hat{\mathrm{y}}^{+}$ & LATE & $\mathrm{n}$ \\
\hline 1 & 0,24 & $-0,07$ & $\begin{array}{l}-0,30^{\star *} \\
(0,14)\end{array}$ & 42 & 0,77 & 0,40 & $\begin{array}{l}-0,37 \\
(0,55)\end{array}$ & 35 \\
\hline 2 & 0,23 & 0,41 & $\begin{array}{c}0,18 \\
(0,12)\end{array}$ & 85 & 0,63 & 0,34 & $\begin{array}{l}-0,29 \\
(0,36)\end{array}$ & 71 \\
\hline 3 & 0,21 & 0,17 & $\begin{array}{l}-0,04 \\
(0,13)\end{array}$ & 132 & 0,59 & 0,49 & $\begin{array}{l}-0,10 \\
(0,33)\end{array}$ & 114 \\
\hline 4 & 0,20 & $-0,68$ & $\begin{array}{l}-0,88 \\
(0,85)\end{array}$ & 165 & 0,56 & 0,44 & $\begin{array}{l}-0,12 \\
(0,32)\end{array}$ & 151 \\
\hline 5 & 0,19 & $-0,22$ & $\begin{array}{l}-0,41 \\
(0,41)\end{array}$ & 204 & 0,54 & 0,46 & $\begin{array}{l}-0,08 \\
(0,28)\end{array}$ & 192 \\
\hline
\end{tabular}

Quelle: LIAB, eigene Berechnungen, ${ }^{* * *} 1 \%,{ }^{* *} 5 \%,{ }^{*} 10 \%$ Signifikanzniveau

Tab. 8: $\quad$ LATE Schätzungen der Anzahl der Einstellungen von 50-Jährigen und Älteren von $6 / 98$ bis $6 / 99$ und von $6 / 98$ bis $6 / 01$ (geschätzte Std. abw. in Klammern)

\begin{tabular}{|c|c|c|c|c|c|c|c|c|}
\hline \multirow[b]{2}{*}{ Bandbreite } & \multicolumn{4}{|c|}{$\begin{array}{c}\text { Einstellungen } \\
\text { zwischen } 6 / 98 \text { und 6/99 }\end{array}$} & \multicolumn{4}{|c|}{$\begin{array}{c}\text { Einstellungen } \\
\text { zwischen } 6 / 98 \text { und } 6 / 01\end{array}$} \\
\hline & $\hat{\mathrm{y}}^{-}$ & $\hat{\mathrm{y}}^{+}$ & LATE & $n$ & $\hat{\mathrm{y}}^{-}$ & $\hat{\mathrm{y}}^{+}$ & LATE & $\mathrm{n}$ \\
\hline 1 & 0,04 & 0,03 & $\begin{array}{l}-0,01 \\
(0,54)\end{array}$ & 58 & 0,41 & 0,02 & $\begin{array}{l}-0,39 \\
(0,45)\end{array}$ & 58 \\
\hline 2 & 0,13 & 0,01 & $\begin{array}{l}-0,12 \\
(0,20)\end{array}$ & 143 & 0,49 & 0,04 & $\begin{array}{l}-0,44^{*} \\
(0,27)\end{array}$ & 123 \\
\hline 3 & 0,15 & 0,00 & $\begin{array}{l}-0,15 \\
(0,17)\end{array}$ & 232 & 0,49 & 0,04 & $\begin{array}{l}-0,45^{\star *} \\
(0,23)\end{array}$ & 192 \\
\hline 4 & 0,16 & $-0,04$ & $\begin{array}{l}-0,20 \\
(0,19)\end{array}$ & 322 & 0,49 & $-0,27$ & $\begin{array}{l}-0,76 \\
(0,58)\end{array}$ & 273 \\
\hline 5 & 0,16 & 0,03 & $\begin{array}{l}-0,12 \\
(0,15)\end{array}$ & 422 & 0,49 & $-0,19$ & $\begin{array}{l}-0,68 \\
(0,44)\end{array}$ & 356 \\
\hline
\end{tabular}

Quelle: LIAB, eigene Berechnungen, ${ }^{* * *} 1 \%,{ }^{* *} 5 \%,{ }^{*} 10 \%$ Signifikanzniveau

Solche Einschränkungen können für die zweite untersuchte Gesetzesänderung nicht ins Feld geführt werden. Die geschätzten Auswirkungen der Neuregelung des Schwellenwertes Ende 1998 ist in Tab. 8 und Tab. 9 dargestellt. In der Spalte LATE finden sich die geschätzten Treatment-Effekte für die Einstellung von Älteren zwischen Juni 1998 und den Monaten Juni 1999 bis $2002^{67}$ bei dem dann geltenden Schwellenwert von fünf Beschäftigten.

Allerdings kommt es auch nach dieser zweiten Änderung nicht zu den erwarteten Effekten. Unabhängig von der Wahl der Bandbreite und des Untersuchungszeitraumes ließen sich keine Hinweise darauf finden, dass der Schwellenwert die neuen Kleinbetriebe von der Einstellung Älterer abhielt. Im Gegenteil weisen alle geschätzten Treatment-Effekte negative Vorzeichen

\footnotetext{
${ }^{67}$ Eine Berechnung der Ergebnisse für den Zeitraum bis 6/2000 ließ sich aufgrund der Datenlage nicht durchführen. Daten für das Jahr 2003 waren zum Zeitpunkte der Berechnung noch nicht im LIAB bereitgestellt.
} 
auf. Vereinzelt ergeben sich für einzelne Bandbreiten und Zeiträume sogar schwach signifikant negative Vorzeichen der Parameter.

Tab. 9: $\quad$ LATE Schätzungen der Anzahl der Einstellungen von 50-Jährigen und Älteren von 6/98 bis 6/02 (geschätzte Std. abw. in Klammern)

\begin{tabular}{|c|c|c|c|c|}
\hline \multirow[b]{2}{*}{ Bandbreite } & \multicolumn{4}{|c|}{$\begin{array}{c}\text { Einstellungen } \\
\text { zwischen } 6 / 98 \text { und } 6 / 02\end{array}$} \\
\hline & $\hat{y}^{-}$ & $\hat{y}^{+}$ & LATE & $n$ \\
\hline 1 & 0,46 & 0,12 & $\begin{array}{l}-0,34 \\
(0,79)\end{array}$ & 40 \\
\hline 2 & 0,60 & 0,03 & $\begin{array}{l}-0,57 \\
(0,37)\end{array}$ & 85 \\
\hline 3 & 0,62 & $-0,05$ & $\begin{array}{l}-0,70^{*} \\
(0,36)\end{array}$ & 131 \\
\hline 4 & 0,63 & $-0,90$ & $\begin{array}{l}-1,53 \\
(1,67)\end{array}$ & 187 \\
\hline 5 & 0,63 & $-0,65$ & $\begin{array}{l}-1,28 \\
(1,22)\end{array}$ & 252 \\
\hline
\end{tabular}

Daraus lässt sich ablesen, dass die Kleinbetriebe, die nun Gefahr laufen, unter das KSchG zu fallen, sogar mehr Neueinstellungen Älterer vornehmen als ihre Nachbarn mit marginal mehr Beschäftigten. Mit dem Ergebnis widerspricht die Empirie allen theoretischen Überlegungen. Allerdings sind die Schätzungen mit den höchsten Bandbreiten und den höchsten Fallzahlen auch diejenigen, die keine signifikanten Vorzeichen für den lokalen Effekt anzeigen.

Zusammenfassend lässt sich wieder sagen, dass die Ergebnisse als weitgehend robust gegenüber der Wahl der Bandbreite und des Untersuchungszeitraumes sind. Die Hypothese, nach der Ältere in Kleinbetrieben keine neue Beschäftigung finden, weil der Schwellenwert des Kündigungsschutzgesetzes Betriebe an der Einstellung hindert, ließ sich nicht bestätigen.

Dennoch sollten die folgenden drei Hinweise in Auge behalten werden: Erstens kann das KSchG indirekt auf die Kontroll- und Treatmentgruppe einwirken. Dadurch dass sich Betriebe unterhalb der Schwelle möglicherweise mit Einstellungen zurückhalten, könnten sich Betriebe über der Schwelle in eine günstigere Lage für Einstellungen versetzt sehen. In diesem Fall beschreibt die Kontrollgruppe nicht den kontrafaktischen Fall einer Welt, in der die Schwelle gar nicht existiert. Da vom Schwellenwert nur eine geringe Anzahl an Betrieben betroffen ist und aufgrund anderer Formen der Regulierung des deutschen Arbeitsmarktes wiegt ein solcher Einwand allerdings weniger schwer.

Ein weiterer Punkt sind die direkten Folgen des Schwellenwertes: Betriebe, die zum Zeitpunkt der Gesetzesänderung schon in den Geltungsbereich des Gesetzes gewachsen sind, können bestrebt sein, ihrerseits auch um den Preis einer teuren Entlassung und trotz der positiven Nachfrageentwicklung unter den Schwellenwert zu schrumpfen und damit ebenfalls von der Maßnahme in ihrem Einstellungsverhalten beeinflusst sein. 
Zuletzt sollte das Ergebnis der Studie von Verick (2004) einfließen, nach der Entscheidungsträger nur unzureichend über die gesetzlichen Details und die Berechnung der kritischen Zahl von Mitarbeitern informiert sind. In diesem Fall könnten sich Betriebe kurz vor dem Schwellenwert nicht in der erwarteten Art und Weise verhalten, da sie sich bereits als große Betriebe im Sinne des KSchG wähnen. Um die Möglichkeit unvollständiger Information zu berücksichtigen, könnte mit einem geeigneten Datensatz, der auch Informationen über die Selbsteinschätzung des Betriebs und seiner Entscheidungsträger enthält, ein fuzzy RD Design verwendet werden. Dann könnten mehrere Diskontinuitäten in der Beziehung zwischen BetriebsgröBe und vermeintlicher Zugehörigkeit zur vom KSchG Betroffener als Quelle der Identifikation verwendet werden.

\section{B 5 Zusammenfassung: Einstellungschancen von Älteren - wie wirkt der Schwellenwert im Kündigungsschutzgesetz}

Es bleibt eine politische Entscheidung, ob das deutsche Kündigungsschutzrecht weiter dereguliert werden soll. Verbunden wird damit die Hoffnung, dass Kleinbetriebe vermehrt Einstellungen vornehmen und so das Problem hoher Arbeitslosigkeit gelindert werden kann. Das Ergebnis dieser Arbeit veranlasst nicht zu solch optimistischen Erwartungen. Trotz der genannten Einschränkungen legen die Ergebnisse den Schluss nahe, dass mit einer weiteren Lockerung allein keine bedeutenden Beschäftigungszuwächse in Kleinbetrieben zu erwarten sind. Die in der Losung „Lieber Arbeit ohne Kündigungsschutz als arbeitslos mit Kündigungsschutz“ suggerierte Wahl zwischen neuer Beschäftigungsdynamik und bestehenden Rechten von Arbeitnehmern scheint so nicht zu bestehen.

Diese Aussage ließ sich so auch auf die Gruppe der Älteren übertragen. Auch für sie konnten keine die Einstellungschancen schmälernden Effekte des Schwellenwertes festgestellt werden. Zudem hat der Gesetzgeber in der Zwischenzeit mit den Hartz-Reformen weitere Maßnahmen ergriffen, die die zu erwartenden Kosten einer Trennung vom älteren Arbeitnehmer trotz geltenden Kündigungsschutzes minimiert: So ermöglicht es der $\S 14$ Abs. 3 des Teilzeit- und Befristungsgesetzes, dass der Arbeitnehmer statt ab 58 Jahren jetzt schon von 52 Jahren an ohne sachlichen Grund befristet beschäftigt wird. Für Jüngere ist dies nur für höchstens zwei Jahre zulässig. De facto können Arbeitskräfte daher schon ab 50 Jahren mehrfach befristet eingestellt werden. Eine einfache und kostengünstige Politikalternative wie sie mit einer weiteren Lockerung der Kündigungsschutzregeln zur Verfügung stünde, scheint nach diesen Ergebnissen keinen Erfolg auf dem Arbeitsmarkt für Ältere zu versprechen. 


\section{Betriebliche Weiterbildung und betriebli- che Altersstruktur ${ }^{68}$}

Ein weiterer, im ersten Kapitel nicht behandelter Aspekt betrieblicher Rahmenbedingungen am Ende des Erwerbslebens ist der der betrieblichen Weiterbildung. Aufgrund der mangelnden Kontinuität der Erhebung des Merkmals im IAB-Betriebspanel musste es ausgespart werden. Auch kann in der folgenden separaten Betrachtung des Themas das Problem der Endogenität der betrieblichen Weiterbildungsentscheidung direkt adressiert werden. Die Ausklammerung in Kapitel A war vor allem deshalb unbefriedigend, weil gerade die Weiterbildung im Betrieb eine besonders viel versprechende Form der Qualifizierung darstellt, wird dabei doch in Humankapital entsprechend den Bedürfnissen der nachfragenden Betriebe investiert.

\section{1 Wirkungen betrieblicher Weiterbildung in der Diskussion}

In den vergangenen Jahren wurden staatliche Qualifizierungsmaßnahmen der aktiven Arbeitsmarktpolitik in Deutschland verstärkt wissenschaftlich evaluiert. Die Ergebnisse der Untersuchungen sind zumeist ernüchternd: So ließen sich kaum positive Effekte auf die Wiederbeschäftigungschancen Arbeitsloser nachweisen. Betriebliche Weiterbildung ist hingegen bisher seltener untersucht worden. Dabei lässt sich vermuten, dass sie einerseits die Flexibilität des Arbeitsangebots bei veränderter Qualifikationsnachfrage erhöht und andererseits die Anpassungsfähigkeit der Betriebe an die Notwendigkeiten neuer Produktionsformen steigert. In Zeiten raschen Verfalls von Humankapital kommt der betrieblichen Weiterbildung somit eine wachsende Bedeutung zu. Sie nimmt eine prophylaktische Funktion am Arbeitsmarkt ein. Zudem stehen Unternehmen angesichts nahender und bereits eingetretener demografischer Veränderungen vor dem Problem, Nachfrage nach bestimmten Qualifikationen in geringerem Maße durch Einstellungen geeigneter Beschäftigter extern decken zu können. Sie müssen stattdessen dazu übergehen, die Qualifikationen der eigenen Beschäftigten diesen Anforderungen anzupassen und möglicherweise auch ältere Beschäftigte länger in den Betrieb einzubinden.

Welche Potentiale in beruflicher Weiterbildung gerade Älterer hierzulande bieten, lassen Daten der Europäischen Kommission vermuten. So lagen die Beteiligungsraten der Erwerbsbevölkerung an beruflicher Weiterbildung zwischen 1998 und 2002 besonders in den Altersgruppen ab 35 Jahren in Deutschland deutlich unter dem EU-Durchschnitt. Die Abweichun-

\footnotetext{
${ }^{68}$ Das folgende Kapitel ist in abgewandelter Fassung als FFB-Diskussionspapier (Burgert (2006c)) sowie als RatSWD Research Note (Burgert (2007)) veröffentlicht. Diskutiert wurde der Inhalt auf der Tagung des Rates für Wirtschafts- und Sozialdaten in Wiesbaden 2007 sowie im Forschungskolloquium Freie Berufe, Mittelstand und empirische Wirtschaftsforschung an der Universität Lüneburg.
} 
gen haben sich bis 2002 erheblich vergrößert. ${ }^{69}$ In diesem Kapitel soll daher die Wirkung betrieblicher Weiterbildung auf die Beschäftigung Älterer untersucht werden.

Als Datengrundlage dient dabei wieder das IAB-Betriebspanel, jetzt ergänzt um Angaben der Beschäftigtenstatistik. Um dem Problem einer potentiellen Selektionsverzerrung zu begegnen, wurde ein Matching-Verfahren angewendet. Die Ergebnisse sollen zeigen, ob betriebliche Weiterbildung dazu beitragen kann, Ältere dem Betrieb zu erhalten und so die demografischen Probleme, die Betriebe und Sozialversicherungen gleichermaßen in den kommenden Jahren treffen werden, zu mildern.

Die bisherigen ökonometrischen Analysen zu den Wirkungen betrieblicher Weiterbildung in Deutschland beschränken sich auf wenige Arbeiten. Dabei bestätigen sich die eingangs formulierten Erwartungen: Fitzenberger und Prey (1999) untersuchen auf Basis des SOEP für Westdeutschland den Einfluss beruflicher Weiterbildung auf die Beschäftigungsstabilität und finden eine signifikante Erhöhung der Beschäftigungsstabilität, die sich mit zunehmender Dauer der Weiterbildungsmaßnahme verstärkt. Becker (2000) sowie Becker und Schömann (1999) untersuchen ebenfalls mit SOEP-Daten für Ostdeutschland und mit der Lebensverlaufsstudie des Max-Planck-Instituts für Bildungschancen für Westdeutschland die Auswirkungen von beruflicher Weiterbildung u.a. auf die Beschäftigungssicherheit und die Wiederbeschäftigungschancen. Die Ergebnisse zeigen auch für Ostdeutschland ein deutlich geringeres Risiko, arbeitslos zu werden, sofern an Weiterbildung teilgenommen wurde, und für Männer bessere Reintegrationschancen bei Arbeitslosigkeit. Hübler (1998) untersucht für Ostdeutschland u.a. die Auswirkungen beruflicher Weiterbildung auf die Arbeitsplatzsicherheit auf Basis des Arbeitsmarktmonitors Ost. Die Ergebnisse zeigen, dass sich die Arbeitsplatzsicherheit bei innerbetrieblichen Maßnahmen eindeutig erhöht. Christensen (2001) kommt ebenfalls zu dem Schluss, dass betriebliche Weiterbildung das Entlassungsrisiko sowie das Risiko freiwilliger Kündigung mindert. Eine umfangreiche Analyse beruflicher Weiterbildung auch Älterer liefern Büchel und Pannenberg (2004). Ihre Studie weist allerdings in eine andre Richtung als die erwähnten. Die Autoren zeigen anhand von SOEP-Daten, dass die individuelle Teilnahme von Personen der Altersgruppe 45 bis 64 Jahre an betrieblicher Weiterbildung keine signifikanten Einflüsse auf ihr zukünftiges Arbeitsplatzrisiko ausübt. Ergebnisse aus anderen Ländern wie den USA, den Niederlanden und der Schweiz ${ }^{70}$ weisen darauf hin, dass sich betriebliche Weiterbildung positiv auf die individuelle Lohnentwicklung auswirkt.

Allerdings sind mir keine Studien bekannt, die Auswirkungen von Weiterbildung auf die betriebliche Altersstruktur durchleuchten und so eine mögliche Förderung von Weiterbildung auf Betriebsseite evaluieren. Diese Forschungslücke soll durch das vorliegende Kapitel ge-

\footnotetext{
${ }^{69}$ Europäische Kommission (2003), S. 55 ff. Befunde von Bellmann und Leber (2004 und 2005) weisen ebenfalls auf eine geringe Weiterbildungsbeteiligung Älterer hin, zeigen jedoch starke betriebliche Variation nach Branchen und Betriebsgröße.

${ }^{70}$ Vgl. z.B. Parent (1999), Leuven und Oosterbeek (2004) sowie Gerfin (2004). Einen Überblick über die internationale Literatur bietet Leuven (2005).
} 
schlossen werden. Es gliedert sich in vier Teile: im folgenden Unterkapitel wird der verwendete Datensatz kurz beschrieben. Daran anschließend wird das methodische Problem der Selbstselektion der Weiterbildungsbeteiligung thematisiert und mit dem Matching-Verfahren eine Lösung vorgestellt. Auf eine Darstellung und Interpretation der Ergebnisse folgt eine Zusammenfassung.

\section{2 Datengrundlage Weiterbildung: Linked Employer Employee Datensatz}

Empirische Grundlage für die Studie stellt vornehmlich das IAB-Betriebspanel dar, das wie oben beschrieben in vielen Wellen auch Informationen über betriebliche Weiterbildungsmaßnahmen enthält. ${ }^{71}$ Zusätzlich zu den Angaben über Weiterbildungsaktivitäten auf Betriebsebene wurden diesen Einheiten Informationen über die Altersstruktur zur Zeit der Weiterbildung und im Jahr 2004 über den Linked-Employer-Employee-Datensatz (LIAB) hinzugefügt. Um ein mögliches Missverständnis im Voraus auszuräumen: Die Studie setzt auf der Betriebsebene an und enthält keine personenindividuellen Weiterbildungsinformationen. Die untersuchte Wirkung ist daher die auf die Altersstruktur der Betriebe und nicht die auf die personenindividuelle Beschäftigungswahrscheinlichkeit im Alter.

Da es sich bei der Veränderung der Altersstruktur um einen langwierigen Prozess handelt und um die Folgen von betrieblicher Weiterbildung abschätzen zu können, wurde zwischen den beobachteten Weiterbildungsaktivitäten und der gemessenen Ergebnisvariablen „Anteil der 50-Jährigen und Älteren“ eine möglichst lange Zeitspanne eingeräumt. Die ersten Beobachtungen von Weiterbildung fallen in die erste Panelwelle 1993, eine ausführlichere Befragung zu den Formen betrieblicher Weiterbildung erfolgt im Jahr 1997. Die untersuchte Outcomegröße ist der Älterenanteil im Betrieb des Jahres 2004. Dieser zeitliche Abstand bringt allerdings mit sich, dass sich der Beobachtungsumfang aufgrund von Panelmortalität erheblich reduziert. Für eine erste Auswertung, in der Weiterbildungsaktivität in den Jahren 1993 bis 1995 untersucht wird, können von ursprünglich knapp 4300 befragten Betrieben 810 für die Auswertung verwendet werden. Im zweiten Datensatz, der den Zeitraum 1997 bis 2004 umfasst und nach Weiterbildungsformen differenziert, sind von anfangs knapp 8900 Panelbetrieben schließlich noch 2858 für die Analyse verwendbar. Für diesen stehen - anders als im ersten Datensatz - auch Betriebe aus Ostdeutschland zur Verfügung.

\footnotetext{
${ }^{71}$ Für eine ausführliche Darstellung des IAB-Betriebspanels vgl. Kölling (2000). Anderen Erhebungen, die Aufschluss über betriebliche Weiterbildungsaktivitäten hätten geben können, fehlt entweder die betriebliche Perspektive, sodass die empirische Modellierung der Weiterbildungsentscheidung nicht möglich gewesen wäre. Beispiele sind hier das Berichtssystem Weiterbildung, der Mikrozensus oder das SOEP. Die europaweite „Continuing Vocational Training Survey“ (CVTS) ist zwar eine Unternehmensbefragung, liegt aber erst in inzwischen drei Wellen vor und erhebt nur wenige betriebliche Merkmale.
} 


\section{2.1 Betriebliche Merkmale}

Die zentrale Angabe der betrieblichen Weiterbildung wird seit Beginn der Panels im Jahr 1993 allerdings unregelmäßig erhoben. Für einzelne Jahre sind darunter auch detaillierte Informationen über die Art der Weiterbildung enthalten, was für die vorliegende Arbeit eine vertiefte Analyse ermöglicht. Einschränkend muss erwähnt sein, dass aus den verwendeten Daten nur hervorgeht, ob der betreffende Betrieb Weiterbildung finanziert hat, nicht aber, ob sich die dort Beschäftigten auf eigene Kosten weitergebildet haben. Die zugehörige Frage im IAB-Betriebspanel lautet: „Förderte Ihr Betrieb/Ihre Dienststelle in der ersten Hälfte des Beobachtungsjahres Fort- und Weiterbildungsmaßnahmen?“72 Wie oben erwähnt, lassen die Daten nicht erkennen, welche Personen im Betrieb in den Genuss der Maßnahmen kamen. Dennoch lassen sich politikrelevante Schlüsse aus den Ergebnissen ziehen. Lässt sich doch die Frage beantworten, ob und, wenn ja welche Auswirkung betriebliche Weiterbildung auf die Altersstruktur ausübt und ob daher eine unspezifische, d.h. eine nicht auf Altersgruppen zugeschnittene, betriebliche Förderung von Weiterbildung den gewünschten Effekt auf die Position Älterer auf dem Arbeitsmarkt zeitigt.

Wie sich in Studien zur Weiterbildungsaktivität von Betrieben zeigt, besteht Anlass zu der Vermutung, dass eine große Heterogenität zwischen weiterbildenden Betrieben und solchen besteht, die auf diese Maßnahme verzichten. Die deshalb verwendete Matching-Methode stellt hohe Anforderungen an den Datensatz und Umfang der Merkmale, die Einfluss auf die Weiterbildungsentscheidung ausüben. Das IAB-Betriebspanel hält eine Vielzahl von verwertbaren Betriebsmerkmalen bereit. Zu den oben aufgezählten Merkmalen, die sich schon in früheren Arbeiten als signifikant erwiesen haben, kommen insbesondere solche Variablen, die als alternative Strategien betrieblicher Qualifikationsanpassung angesehen werden können. Zudem enthält das Panel Fragen, die Aufschluss über neu entstandenen Qualifikationsbedarf der Belegschaft geben können, wie Angaben zum Investitionsverhalten der Betriebe.

\section{2.2 Persönliche Merkmale}

Neben den Informationen über die Betriebe ließen sich dem IAB-Panel auch individuelle Merkmale hinzuspielen. Mittels der Betriebsnummer lassen sich mit den Betrieben tagesgenaue Angaben aus der Beschäftigtenstatistik verknüpfen, die die Altersstruktur der Belegschaft offenbaren und so die Beobachtung der Ergebnisvariablen dieser Untersuchung und die Einbeziehung der Altersstruktur zum Zeitpunkt der Weiterbildung möglich machen. Allerdings ist wieder anzumerken, dass eine Zuordnung von Weiterbildungsmaßnahmen im Betrieb zu den dort beschäftigten Personen nicht möglich ist.

\footnotetext{
${ }^{72}$ Ein Zusatz erläutert die Frage weiter: „Das heißt: Wurden Arbeitskräfte zur Teilnahme an inner- und außerbetrieblichen Maßnahmen freigestellt bzw. wurden die Kosten für Weiterbildungsmaßnahmen ganz oder teilweise vom Betrieb übernommen?"
} 


\section{3 Mikroökonometrischer Ansatz: Die Matching-Methode}

Ziel der Studie ist es, den Einfluss betrieblicher Weiterbildung auf die Altersstruktur des Betriebes zu ermitteln. Als zentrales Hindernis in der empirischen Umsetzung stellt sich dabei das endogene Weiterbildungsverhalten der Betriebe dar. So zeigen einige Arbeiten deutliche Unterschiede zwischen Betrieben, die Weiterbildung anbieten, und solchen, die dies nicht tun. Düll und Bellmann (1998) werten das IAB-Panel aus, Gerlach und Jirjahn (1998) das Hannoveraner Firmenpanel. Dabei finden beide Autorenpaare einen positiven Betriebsgrößeneffekt, der sich durch Skaleneffekte bei der Durchführung und einen ausgeprägten internen Arbeitsmarkt erklären lässt. Ebenso macht den Untersuchungen zufolge ein großer Anteil an Hochqualifizierten Weiterbildung im Betrieb wahrscheinlicher. Auch kommen die Autoren zu dem Ergebnis, dass die Existenz eines Betriebsrates die Weiterbildungsneigung erhöht.

Es ist davon auszugehen, dass Merkmale, die die Weiterbildungsentscheidung der Betriebe beeinflussen, direkt auch auf die Personalpolitik gegenüber älteren Beschäftigten wirken. Die Gruppe der Betriebe mit Weiterbildungsmaßnahmen wird sich - wie in den oben aufgeführten Studien belegt und wie im Folgenden gezeigt - in ihren ökonomischen Charakteristika erheblich von der Gruppe der Betriebe unterscheiden, die auf dieses Mittel verzichten. Diese Selektionsverzerrung führt bei einer gewöhnlichen - auch nicht-linearen - Regression zu einer verzerrten Schätzung des Weiterbildungseffekts. Das deshalb an dieser Stelle zu verwendende Verfahren des Matching verfolgt das Ziel, eine adäquate Kontrollgruppe bei solchen, nichtexperimentellen Daten zu finden, um damit den kausalen Effekt betrieblicher Weiterbildung zu bestimmen.

\section{3.1 Evaluationsansatz}

Den Modellrahmen des Evaluationsansatzes stellt das Roy-Rubin-Modell ${ }^{73}$ dar, das im Folgenden kurz eingeführt werden soll ${ }^{74}$ : Darin bezeichnet man die Ergebnisvariable - hier: den Anteil der Über-50-Jährigen des Betriebes i - im Falle einer Maßnahmeteilnahme - hier: bei Finanzierung von betrieblicher Weiterbildung - mit $\mathrm{y}_{1 \mathrm{i}}$ und mit $\mathrm{y}_{0 \mathrm{i}}$ für den Fall, dass Betrieb i kein solches Treatment gewählt hat. Das Evaluationsproblem besteht nun darin, dass ein und derselbe Betrieb nicht in beiden Zuständen und damit der individuelle kausale Effekt

$$
\mathrm{y}_{1 \mathrm{i}}-\mathrm{y}_{0 \mathrm{i}}
$$

nicht beobachtet werden kann. Stattdessen offenbart sich dem Forscher für Betrieb i nur der Älterenanteil $y_{i}$, der sich entweder mit Weiterbildung $\left(D_{i}=1\right)$ oder ohne diese $\left(D_{i}=0\right)$ ergibt:

$$
\mathrm{y}_{\mathrm{i}}=\mathrm{y}_{0 \mathrm{i}}+\mathrm{D}_{\mathrm{i}}\left(\mathrm{y}_{1 \mathrm{i}}-\mathrm{y}_{0 \mathrm{i}}\right) \text { mit } \mathrm{D}_{\mathrm{i}} \in\{0 ; 1\}
$$

\footnotetext{
${ }^{73}$ Die Namensgebung für diese Art des Ansatzes geht zurück auf die Arbeiten von Roy (1951) und Rubin (1974). Alternativ ist in der englischsprachigen Literatur der Begriff potential outcome approach gewöhnlich.

${ }^{74}$ Einen Überblick über mikroökonometrische Evaluationsmethoden bieten Heckman et al. (1999).
} 
Um die Fragstellung zu beantworten, ist es allerdings notwendig, zumindest allgemeine Aussagen über die Differenz zwischen den beiden Zuständen, $\mathrm{y}_{1 \mathrm{i}}-\mathrm{y}_{0 \mathrm{i}}$, und damit über den kausalen Effekt von betrieblicher Beteiligung auf die Altersstruktur der Belegschaft zu treffen. Da der kontrafaktische Zustand sich für einen individuellen Betrieb nicht beobachten lässt, muss auf Mittelwerte der Gesamtpopulation ausgewichen werden. So lässt sich der durchschnittliche Maßnahmeeffekt auf die Teilnehmenden, der so genannte Average Treatment Effect on the Treated (ATT),

$$
\mathrm{ATT}=\mathrm{E}\left[\mathrm{y}_{1}-\mathrm{y}_{0} \mid \mathrm{D}=1\right]=\mathrm{E}\left[\mathrm{y}_{1} \mid \mathrm{D}=1\right]-\mathrm{E}\left[\mathrm{y}_{0} \mid \mathrm{D}=1\right]
$$

unter unten näher zu bestimmenden Annahmen zu schätzen. Er gibt an, um wie viel der erwartete Älterenanteil für die Untergruppe der weiterbildenden Betriebe bei Weiterbildung höher liegt, als es ohne die Maßnahme zu erwarten gewesen wäre. Das Evaluationsproblem besteht nun darin, eine geeignete Kontrollgruppe für die weiterbildenden Betriebe zu finden.

\section{3.2 Identifikation}

Die einfachste Kontrollgruppe der Nicht-Teilnehmer und deren beobachtbare durchschnittliche Produktivität $\mathrm{E}\left[\mathrm{y}_{0} \mid \mathrm{D}=0\right]$ als Schätzwert für $\mathrm{E}\left[\mathrm{y}_{0} \mid \mathrm{D}=1\right] \mathrm{zu}$ verwenden, ist in nichtexperimentellen Studien aufgrund von Selektionsverzerrungen nicht sinnvoll. Eine solche Schätzung des Effektes als

$$
\mathrm{E}\left[\mathrm{y}_{1} \mid \mathrm{D}=1\right]-\mathrm{E}\left[\mathrm{y}_{0} \mid \mathrm{D}=0\right]=\mathrm{ATT}-\mathrm{E}\left[\mathrm{y}_{0} \mid \mathrm{D}=1\right]+\mathrm{E}\left[\mathrm{y}_{0} \mid \mathrm{D}=0\right]
$$

würde nur dann unverzerrt sein, wenn auf der rechten Seite dieser Gleichung die beiden Erwartungswerte übereinstimmten und $\mathrm{E}\left[\mathrm{y}_{0} \mid \mathrm{D}=1\right]=\mathrm{E}\left[\mathrm{y}_{0} \mid \mathrm{D}=0\right]$ gälte. Aber gerade der selbstselektive Charakter der Entscheidung, ob der Betrieb Weiterbildung finanziert, lässt befürchten, dass diejenigen Betriebe, die sich dazu entschließen, eine andere Altersstruktur als die naive Kontrollgruppe aufweisen würden, auch wenn sie keine Weiterbildungsanstrengungen unternähmen.

Eine alternative Schätzmöglichkeit bietet das hier verwendete Matching-Verfahren: Es beruht auf der so genannten Conditional Independence Assumption (CIA). Diese besagt, dass sich der Selektionsprozess in die Programm- und Kontrollgruppe vollständig durch die beobachtbaren Variablen X erklären lässt. Die Outcomevariable bei Nicht-Teilnahme, hier die Altersstruktur, die sich ohne Weiterbildung ergibt, ist dann - sofern die CIA gilt - unabhängig vom Teilnahmestatus $\left(\mathrm{y}_{0} \perp \mathrm{D} \mid \mathrm{X}\right)$, d.h. gegeben den Vektor $\mathrm{X}$ gilt:

$$
\mathrm{E}\left[\mathrm{y}_{0} \mid \mathrm{D}=1, \mathrm{X}\right]=\mathrm{E}\left[\mathrm{y}_{0} \mid \mathrm{D}=0, \mathrm{X}\right] \text {. }
$$

Die CIA rechtfertigt es somit, die kontrafaktische Situation doch mit Betrieben, die keine Weiterbildung finanziert haben, abzubilden. Problematisch bleibt dabei jedoch die Annahme, dass sämtliche relevanten Einflussfaktoren tatsächlich beobachtbar sind. Sie lässt sich nur dann rechtfertigen, wenn man auf einen detaillierten Datensatz zurückgreifen kann, der die relevanten Merkmale der Teilnahmeentscheidung enthält. Die Verwendung von Daten des 
IAB-Betriebspanels ergänzt um individuelle Informationen der Beschäftigten lässt die für das Matching-Verfahren notwendige Annahme gerechtfertigt erscheinen.

Zudem stellt die CIA den Auswerter vor ein praktisches Problem: Mit der Zahl der die Selektion erklärenden Variablen in $\mathrm{X}$ und der Zahl der verschiedenen Ausprägungen von $\mathrm{X}$, die Betriebe in der Treatmentgruppe aufweisen, steigt ebenfalls die Zahl der notwendigen Betriebe in der Kontrollgruppe, und damit die Schwierigkeit, eine Kontrollgruppe zu finden, die in allen relevanten Charakteristika mit der Teilnehmergruppe übereinstimmt. Eine Lösung für diesen „Fluch der Dimensionalität“ haben Rosenbaum und Rubin (1983) aufgezeigt: So reicht es aus, Teilnehmer und Nicht-Teilnehmer anhand eines aus den Variablen in X gebildeten balancing scores $\mathrm{b}(\mathrm{X})$ zuzuordnen und damit das Problem der hohen Dimensionalität zu umgehen. Gegeben diesen Wert sind $\mathrm{y}_{0}$ und $\mathrm{y}_{1}$ unabhängig vom Treatmentstatus und damit die Erwartungswerte der beiden Größen identisch für Betriebe der Kontroll- und Treatmentgruppe:

$$
\mathrm{E}\left[\mathrm{y}_{0} \mid \mathrm{D}=1, \mathrm{~b}(\mathrm{X})\right]=\mathrm{E}\left[\mathrm{y}_{0} \mid \mathrm{D}=0, \mathrm{~b}(\mathrm{X})\right]
$$

Neben dieser abgewandelten Unabhängigkeitsannahme muss bei der Implementation eines Matching-Ansatzes auch die Common-Support-Bedingung berücksichtigt werden. Sie stellt sicher, dass nur solche weiterbildenden Betriebe bei der Schätzung berücksichtigt werden, für die ein passender Betrieb in der Kontrollgruppe gefunden wird. So wurden solche weiterbildenden Betriebe nicht einbezogen, deren balancing score über dem maximalen der nichtweiterbildenden Betriebe lag. Zwar verringert sich dadurch die Aussagekraft der Schätzung, da sich diese nur auf den Common Support bezieht. Diese Einschränkung kann jedoch im betrachteten Fall als wenig restriktiv erachtet werden, da die hier ausgeschlossene Gruppe der Betriebe mit hoher Weiterbildungsneigung auf mögliche politisch gesetzte Anreize einer Förderung von Weiterbildung nur geringe Reaktionen erwarten lässt. Ziel der Schätzung ist es dann, den Wert

$$
\mathrm{ATT}=\mathrm{E}\left(\left[\mathrm{y}_{1} \mid \mathrm{D}=1, \mathrm{~b}(\mathrm{X})\right]-\mathrm{E}\left[\mathrm{y}_{0} \mid \mathrm{D}=0, \mathrm{~b}(\mathrm{X})\right]\right)
$$

zu bestimmen. Die technische Umsetzung der Schätzung stellt den Forscher vor die Wahl zwischen einer Vielzahl von Methoden, die Gegenstand des folgenden Abschnittes sind.

\section{3.3 Spezifikation des Schätzers}

Zunächst muss ein geeigneter Ausgleichswert $b(X)$ als Funktion aus den Selektionsvariablen in X gebildet werden. Die gebräuchlichsten Verfahren der ATT-Schätzung basieren auf der Schätzung des propensity scores in diesem ersten Schritt. Allerdings sind auch Methoden verbreitet, die den balancing score aus anderen Funktionen ableiten. Eine Übersicht über die Eigenschaften und Unterschiede zwischen diesem so genannten Covariate-Matching und dem im Folgenden verwendeten Propensity-Score-Matching findet sich bei Zhao (2004). 
In einem ersten Schritt ist daher die Teilnahmeneigung des einzelnen Betriebs zu schätzen. Caliendo und Kopeinig (2005) weisen darauf hin, dass die Wahl der funktionalen Form dieser Schätzung recht unbedeutend ist, da sich die beiden klassischen Spezifikationen des Logit bzw. Probit nur marginal in ihren geschätzten Wahrscheinlichkeiten des Eintritts unterscheiden. Einzig das lineare Wahrscheinlichkeitsmodell wird aufgrund seiner bekannten Unzulänglichkeiten nicht in Betracht gezogen (vgl. z.B. Greene (2003) 665f.).

Was die Auswahl der in der Propensity-Score-Schätzung verwendeten Kovariablen betrifft, stehen unterschiedliche Strategien zur Auswahl. Bryson et al. (2002) argumentieren, dass die Einbeziehung von Regressoren, die nicht zur Sicherstellung der CIA-Annahme notwendig sind, zwar nicht verzerrend wirkt, aber die Varianz der Schätzung erhöht. Allerdings plädieren Rubin und Thomas (1996) auch angesichts der Tatsache, dass sich die entscheidende Bedingung der CIA nicht empirisch belegen lässt, für eine weniger zurückhaltende Modellauswahl. Sie verweisen darauf, dass Variablen nur dann ausgeschlossen werden sollen, wenn es als sicher angesehen werden kann, dass die Variable die Outcomegröße y nicht beeinflusst.

Die hier vorgenommene Auswahl der Regressoren lehnt sich an die zitierten Vorarbeiten an und bezieht mit der Altersstruktur, wie sie zu Beginn des Weiterbildungszeitraumes beobachtet wurde, eine in diesem Zusammenhang wichtige Angabe mit ein. Eine Kontrolle von Branche und Betriebsgröße in der Klassifizierung, wie sie für die geschichtete Stichprobenziehung des IAB-Betriebspanels maßgeblich ist, soll Selektionsverzerrungen beheben, die sich aus der Datenerhebung ergeben könnten.

Eine weitere Spezifikation des Matching-Algorithmus muss vorgenommen werden bezüglich der Auswahl und Gewichtung von Elementen der Kontrollgruppe, die dem einzelnen weiterbildenden Betrieb aus der Treatmentgruppe gegenübergestellt werden soll. Dabei lassen sich verschiedene Verfahren unterscheiden, die jeweils eine Abwägung verlangen zwischen hinzunehmender Verzerrung, die sich aus Abweichungen in den kontrollierten Werten des balancing score ergeben, und zuzulassender Varianz der Effekt-Schätzung: Um die Allgemeingültigkeit der Ergebnisse zu unterstreichen, wurden sowohl ein Nearest-NeighbourMatching als auch ein Local-Linear-Matching vorgenommen. Im ersten Fall ordnet man jedem teilnehmenden Betrieb denjenigen zu, der den nächsten propensity score aufweist. Dabei können einzelne Kontroll-Betriebe entweder nur ein einziges Mal aus Vergleich dienen oder aber mehrmals ausgewählt werden. Aufgrund der besonders im ersten Datensatz geringen Anzahl an vergleichbaren Beobachtungen in der Kontrollgruppe wurde ein Zurücklegen zugelassen. Im zweiten Fall des Local-Linear-Matching werden die Kontrollbetriebe durch eine lokale lineare Regression anhand der ermittelten propensity scores ermittelt. Die Methode stellt mit ihrer Einbeziehung einer Vielzahl von Kontrollbetrieben ein anderes Extrem unter den zur Verfügung stehenden Matching-Algorithmen dar.

Asymptotisch sind die verschiedenen Algorithmen identisch. In endlichen Stichproben kann es bei den Schätzungen allerdings zu Unterschieden kommen, die sich in der vorliegenden Studie als geringfügig herausstellten. 


\section{4 Mikroökonometrische Ergebnisse}

Mit dem Ausmaß und der Verteilung von betrieblicher Weiterbildung nach der Altersstruktur in der Stichprobe soll die Frage der Bestimmungsgründe für Weiterbildung zunächst deskriptiv beleuchtet und mit Ergebnissen anderer Studien verglichen werden. Nach einer Diskussion der Qualität der verwendeten Matching-Methoden schließen sich die Ergebnisse der kausalanalytischen Untersuchungen an.

Eine erste, rein deskriptive Aussage stellt die Weiterbildungsbeteiligung der verwendeten BPBetriebe dar. Darin ist von in den Jahren 1993 bis 1995 in 410 von 810 Fällen eine durchgängige Weiterbildungsfinanzierung seitens des Betriebs festzustellen gewesen. In der Panelbefragung des Jahres 1997 gaben knapp 72 \% der Betriebe an, mindestens eine der dann folgenden Formen betrieblicher Weiterbildung finanziert zu haben (vgl. Abb. 25). Externe Kurse und Vorträge, die in einer Mehrzahl der Betriebe zur Anwendung kamen, bilden dabei die beliebtesten Weiterbildungsformen. Danach folgen mit internen Kursen und Weiterbildung am Arbeitsplatz Arten der Fortbildung, für die nicht auf betriebsexterne Ressourcen zurückgegriffen werden muss. Ein Schattendasein fristen die Jobrotation und Qualitätszirkel sowie selbst gesteuertes Lernen, die jeweils von einer deutlichen Minderheit der Betriebe genutzt werden.

Abb. 25: $\quad$ Anteile betrieblicher Weiterbildung 1997 nach Art der Weiterbildung

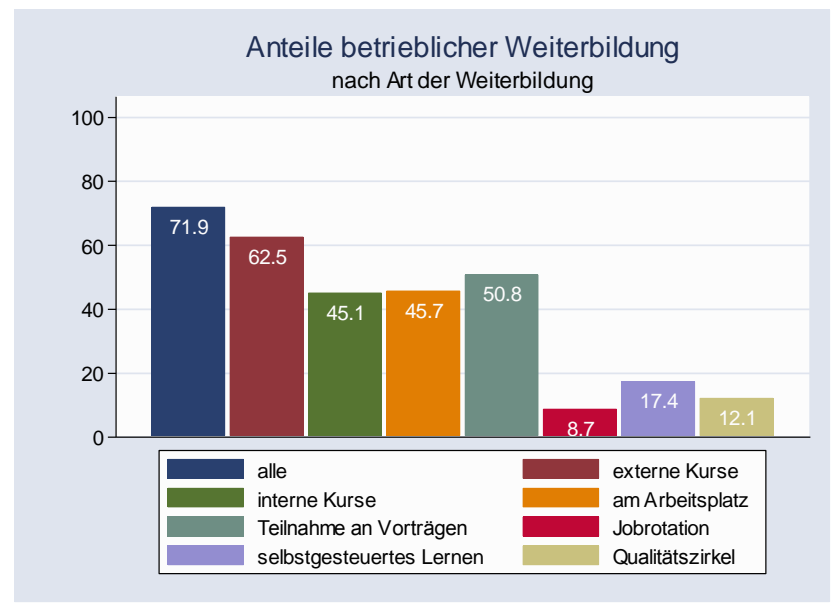

Quelle: IAB-Betriebspanel Welle 1997

Verglichen mit Untersuchungen, in denen personenbezogene Daten ausgewertet werden, sind die $72 \%$ ein sehr hoher Wert und deuten darauf hin, dass innerhalb der Betriebe eine restriktive Auswahl der teilnehmenden Beschäftigten vorgenommen wird.

Um einen ersten Ausblick auf den Hauptauswertungszweck der Untersuchung zu wagen, ist in Anhang C

die Altersstruktur von Betrieben dargestellt, die in den Jahren 1993 bis 1995 durchgängig Weiterbildung finanziert haben und der Altersverteilung derjenigen Betriebe gegenübergestellt, die in mindestens einem der Jahre auf diese Form der Qualifikationsanpassung verzich- 
tet haben. Dabei lassen sich signifikante, aber der Größenordnung nach unbedeutende Unterschiede zwischen den beiden Gruppen finden: In den weiterbildenden Betrieben sind jeweils die Anteile der Älteren, aber auch der Jüngeren geringer als in der Vergleichsgruppe.

Abb. 26: $\quad$ Betriebliche Altersstruktur 2004 nach Weiterbildung 1993 bis 1995

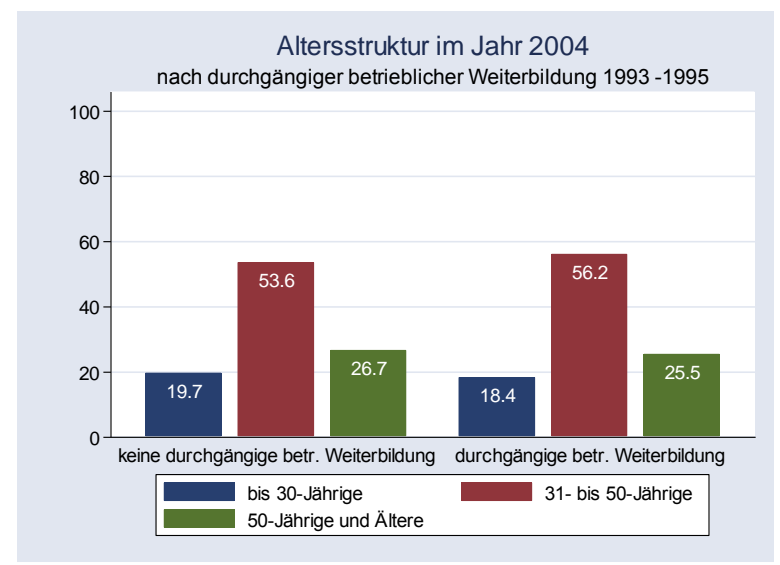

Quelle: LIAB-Querschnitt Wellen 1993 - 2004

Abb. 27:

Betriebliche Altersstruktur 2004 nach Weiterbildung 1997

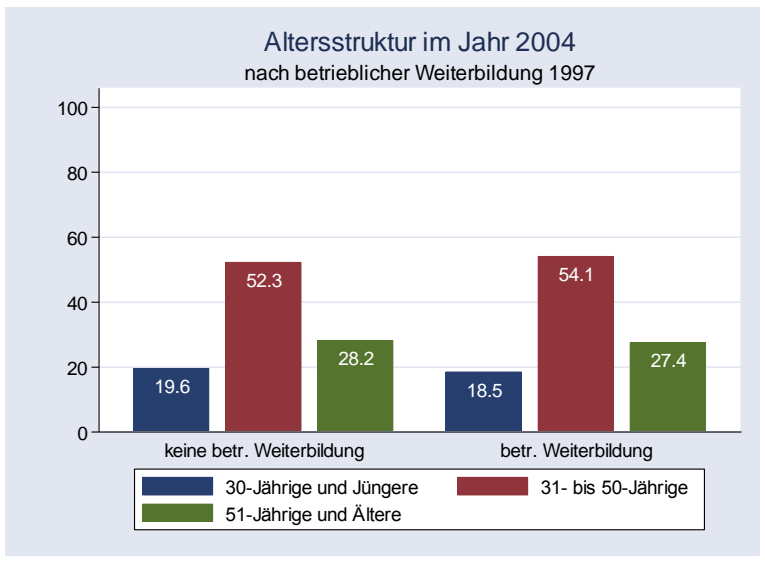

Quelle: LIAB-Querschnitt Wellen 1997 - 2004

Dies gilt für die Finanzierung von Weiterbildung im beiden verwendeten Datensätze, wie aus den Abbildungen Abb. 27 ersichtlich ist. Zwischen den beiden Gruppen lassen sich nur unbedeutende Unterschiede im Anteil der Älteren feststellen. Dies gilt für die allgemeine Frage nach Finanzierung betrieblicher Weiterbildung ebenso wie für die nach speziellen Formen der Qualifizierung (vgl. Abb. 37 bisAbb. 39). Für jeden der Vergleiche lässt sich ein geringfügig geringerer Anteil der Älteren für die aktiven Betriebe feststellen. Allerdings wurde hier noch nicht für Selektionseffekte kontrolliert, sodass sich eine kausale Interpretation der Vergleiche verbietet. Diese Kontrolle mittels des beschriebenen Matching-Ansatzes ist Gegenstand der folgenden Abschnitte. 


\section{4.1 Ergebnisse der Propensity-Score-Schätzung}

Die Schätzung des balancing scores und damit der Bestimmungsgründe der Finanzierung von betrieblicher Weiterbildung ist in den Tabellen Tab. 10 und Tab. 11, sowie Tab. 15 bis Tab. 17 im Anhang C dargestellt.

Aus humankapitaltheoretischer Sicht übt die aktuelle Altersstruktur des Betriebes Einfluss auf die Weiterbildungsentscheidung aus. ${ }^{75}$ Dies findet sich auch in den Ergebnissen für alle Schätzung wieder: Gegenüber der Referenzkategorie der bis 25-Jährigen weist die große Mehrheit der Altersgruppendummies ein negatives Vorzeichen auf, auch wenn nur wenige der ermittelten Koeffizienten signifikanten Einfluss besitzen - ein Umstand der möglicherweise der Vielzahl von Altersklassen zuzuschreiben ist. ${ }^{76}$

Deutliche Unterschiede in der Weiterbildungsaktivität gehen bei den Schätzungen 1997 auch von der Branche aus. Im Einklang mit früheren Untersuchungen (vgl. z.B. Düll und Bellmann (1998) 213f.) erweist sich die Zugehörigkeit zu einer Branche als wichtiger Faktor zur Bestimmung der Weiterbildungsneigung. Gegenüber der Referenz „Land- und Forstwirtschaft“ neigt die Mehrheit der Wirtschaftszweige in den Schätzungen dieses zweiten Datensatzes zu einer signifikant höheren Weiterbildungsbeteiligung.

Ebenso erweist sich die Anzahl der Beschäftigten als hochsignifikanter Prädikator für die abhängige Variable. Generell lässt sich sagen, dass in größeren Betrieben eine ebenso größere Wahrscheinlichkeit der Finanzierung von betrieblicher Weiterbildung besteht. In einigen Schätzungen musste die Klasse der Betriebe mit 5000 und mehr Beschäftigten gar wegen Kollinearität aus der Schätzung ausgeschlossen werden, da ausschließlich Betriebe der Treatmentgruppe darin enthalten waren.

Ein offensichtlicher Anlass für die Notwendigkeit von Weiterbildung liegt in der Änderung der technischen Ausstattung eines Betriebes. Die zugehörige Variable, mit der der Zustand der technischen Anlagen als neu bzw. sehr neu eingeschätzt wurde, erhält für alle Arten der Weiterbildung 1997 ein hochsignifikantes positives Vorzeichen und bestätigt damit die Hypothese. Ebenso wirken in allen Regressionen Investitionen in Kommunikationstechnik hoch signifikant positiv. Die Ergebnisse decken sich wieder mit den Ergebnissen früherer Studien (vgl. z.B. Düll und Bellmann (1998)).

Ein ebenso häufig festgestellter Befund empirischer Arbeiten spiegelt sich auch in den hier gefundenen Ergebnissen wider: Mit steigendem Anteil an qualifizierten Beschäftigten erhöht sich auch die Weiterbildungsneigung der Betriebe. Dies deckt sich mit Erkenntnissen, dass ohnehin schon Hochqualifizierte einen leichteren Zugang zu Weiterbildung haben (vgl. Büchel und Pannenberg (2005)).

\footnotetext{
${ }^{75}$ So berichten z.B. Bellmann und Leber (2004), dass im Jahr 2002 nur 6\% der westdeutschen und 7\% der ostdeutschen Betriebe ältere Beschäftigte an Weiterbildungsmaßnahmen beteiligen.

${ }^{76}$ Eine frühere Version dieses Papers ergab tatsächlich für eine einzige Altersgruppe der Über-50-Jährigen einen signifikant negativen Einfluss auf die durchgängige Weiterbildung in den Jahren 1993 bis 1995.
} 
Tab. 10: Probit-Schätzung der Finanzierung von Weiterbildung 1993

\begin{tabular}{|c|c|c|c|c|}
\hline \multicolumn{2}{|c|}{ Weiterbildung 1993 bis1995 } & Koeffizient & $\mathbf{Z}$ & $P>|z|$ \\
\hline \multicolumn{2}{|c|}{ Altersstruktur 1993 (in \%) } & \multicolumn{3}{|c|}{ Referenzkategorie: Anteil bis 25-Jährige } \\
\hline \multicolumn{2}{|c|}{ Anteil 26- bis 30-Jährige } & 0,082 & 0,11 & 0,912 \\
\hline \multicolumn{2}{|c|}{ Anteil 31- bis 35-Jährige } & $-0,179$ & $-0,22$ & 0,824 \\
\hline \multicolumn{2}{|c|}{ Anteil 36- bis 40-Jährige } & $-0,138$ & $-0,16$ & 0,871 \\
\hline \multicolumn{2}{|c|}{ Anteil 41- bis 45-Jährige } & 0,882 & 1,14 & 0,253 \\
\hline \multicolumn{2}{|c|}{ Anteil 46- bis 50 -Jährige } & 0,184 & 0,19 & 0,851 \\
\hline \multicolumn{2}{|c|}{ Anteil 51- bis 55-Jährige } & $-2,352$ & $-2,06$ & 0,040 \\
\hline \multicolumn{2}{|c|}{ Anteil 56- bis 60-Jährige } & 0,278 & 0,22 & 0,826 \\
\hline \multicolumn{2}{|c|}{ Anteil 61- bis 65-Jährige } & 2,618 & 0,84 & 0,401 \\
\hline \multicolumn{2}{|c|}{ Anteil über 65-Jährige } & $-5,091$ & $-1,08$ & 0,279 \\
\hline \multicolumn{2}{|c|}{ Branche (Dummy) } & \multicolumn{3}{|c|}{ Referenzkategorie: Land- und Forstwirtschaft } \\
\hline \multicolumn{2}{|c|}{ Energie und Bergbau } & 0,640 & 0,93 & 0,350 \\
\hline \multicolumn{2}{|c|}{ Grundstoffverarbeitung } & 0,300 & 0,54 & 0,590 \\
\hline \multicolumn{2}{|c|}{ Investitionsgüter } & 0,415 & 0,76 & 0,446 \\
\hline \multicolumn{2}{|c|}{ Verbrauchsgüter } & $-0,300$ & $-0,53$ & 0,593 \\
\hline \multicolumn{2}{|c|}{ Baugewerbe } & $-0,055$ & $-0,09$ & 0,925 \\
\hline \multicolumn{2}{|l|}{ Handel } & $-0,327$ & $-0,58$ & 0,560 \\
\hline \multicolumn{2}{|c|}{ Verkehr und Nachrichten } & $-0,048$ & $-0,08$ & 0,938 \\
\hline \multicolumn{2}{|c|}{ Kreditgewerbe } & 0,524 & 0,81 & 0,419 \\
\hline \multicolumn{2}{|l|}{ Versicherungen } & $-0,465$ & $-0,64$ & 0,522 \\
\hline Gaststätten un & leime & $-0,059$ & $-0,10$ & 0,922 \\
\hline Bildung und $\mathrm{Ve}$ & & 0,202 & 0,33 & 0,743 \\
\hline Gesundheitswe & & 0,191 & 0,31 & 0,755 \\
\hline Freie Berufe u. & & $-0,098$ & $-0,16$ & 0,872 \\
\hline Sonstige Diens & istungen & $-0,963$ & $-1,10$ & 0,272 \\
\hline Organisationen & nne Erwerbscharakter/Staat & $-0,132$ & $-0,24$ & 0,812 \\
\hline Anteil qualifiz & te Beschäftigte (in \%) & 0,987 & 3,76 & 0,000 \\
\hline Anteil weiblicl & Beschäftigte (in \%) & 0,383 & 1,29 & 0,197 \\
\hline Anteil Auszub & ende (in \%) & 0,866 & 0,82 & 0,411 \\
\hline Betriebsgröße & ummy) & Referenzká & $100 \mathrm{~b}$ & chäftigte \\
\hline 1 bis 4 Beschä & & $-1,775$ & $-3,67$ & 0,000 \\
\hline 5 bis 9 Beschä & & $-1,137$ & $-3,60$ & 0,000 \\
\hline 10 bis 19 Besc & tigte & $-1,073$ & $-3,47$ & 0,001 \\
\hline 20 bis 49 Besc & tigte & $-0,310$ & $-1,33$ & 0,182 \\
\hline 50 bis 99 Besc & tigte & $-0,271$ & $-1,17$ & 0,242 \\
\hline 200 bis $499 \mathrm{Be}$ & רäftigte & 0,241 & 1,13 & 0,257 \\
\hline 500 bis $999 \mathrm{Be}$ & läftigte & 0,761 & 2,78 & 0,005 \\
\hline 1000 bis 4999 & schäftigte & 0,732 & 3,37 & 0,001 \\
\hline 5000 u. m. Bes & äftigte & 1,171 & 2,16 & 0,031 \\
\hline Technische A & gen auf neuem Stand (Dummy) & 0,069 & 0,52 & 0,604 \\
\hline Investitionen i & Kommunikationstechnik (Dummy) & 0,376 & 2,89 & 0,004 \\
\hline Überstunden & Immy) & 0,392 & 3,04 & 0,002 \\
\hline Fachkräftema & el (Dummy) & 0,277 & 2,27 & 0,023 \\
\hline Betriebsrat (D & imy) & 0,159 & 0,87 & 0,384 \\
\hline Konstante & & $-1,159$ & $-1,51$ & 0,131 \\
\hline $\begin{array}{l}\text { N } \\
\text { LR chi2(41) } \\
\text { Prob > chi2 } \\
\text { Log likelihood } \\
\text { Pseudo R2 }\end{array}$ & $\begin{array}{l}781 \\
414,88 \\
0,0000 \\
333,72245 \\
0,3833\end{array}$ & & & \\
\hline
\end{tabular}

Quelle: LIAB-Längsschnitt, Wellen 1993 bis 2004, eigene Berechnungen 
Tab. 11: $\quad$ Probit-Schätzung der Finanzierung von Weiterbildung 1997

\begin{tabular}{|c|c|c|c|}
\hline Weiterbildung 1997 & Koeffizient & $\mathbf{Z}$ & $P>|z|$ \\
\hline Altersstruktur 1997 (in \%) & \multicolumn{3}{|c|}{ Referenzkategorie: Anteil bis 25-Jährige } \\
\hline Anteil 26- bis 30-Jährige & $-0,253$ & $-0,97$ & 0,330 \\
\hline Anteil 31- bis 35-Jährige & $-0,285$ & $-1,15$ & 0,249 \\
\hline Anteil 36- bis 40-Jährige & $-0,830$ & $-3,20$ & 0,001 \\
\hline Anteil 41- bis 45-Jährige & $-0,436$ & $-1,61$ & 0,107 \\
\hline Anteil 46 - bis 50 -Jährige & $-0,647$ & $-2,45$ & 0,014 \\
\hline Anteil 51- bis 55-Jährige & 0,044 & 0,14 & 0,889 \\
\hline Anteil 56- bis 60-Jährige & $-0,672$ & $-1,92$ & 0,055 \\
\hline Anteil 61- bis 65-Jährige & $-1,421$ & $-1,52$ & 0,129 \\
\hline Anteil über 65-Jährige & $-2,017$ & $-1,08$ & 0,282 \\
\hline Branche (Dummy) & \multicolumn{3}{|c|}{ Referenzkategorie: Land- und Forstwirtschaf } \\
\hline Energie und Bergbau & 1,667 & 3,35 & 0,001 \\
\hline Grundstoffverarbeitung & 0,427 & 2,21 & 0,027 \\
\hline Investitionsgüter & 0,590 & 3,45 & 0,001 \\
\hline Verbrauchsgüter & 0,182 & 1,03 & 0,305 \\
\hline Baugewerbe & 0,176 & 1,05 & 0,296 \\
\hline Handel & 0,439 & 2,65 & 0,008 \\
\hline Verkehr und Nachrichten & 0,320 & 1,58 & 0,114 \\
\hline Kreditgewerbe & 1,332 & 2,63 & 0,009 \\
\hline Versicherungen & 0,354 & 0,93 & 0,354 \\
\hline Gaststätten und Heime & 0,533 & 2,96 & 0,003 \\
\hline Bildung und Verlage & 0,504 & 2,42 & 0,016 \\
\hline Gesundheitswesen & 0,690 & 3,41 & 0,001 \\
\hline Freie Berufe u. ä. & 0,254 & 1,40 & 0,161 \\
\hline Sonstige Dienstleistungen & 0,442 & 1,72 & 0,086 \\
\hline Organisationen ohne Erwerbscharakter/Staat & 0,621 & 3,60 & 0,000 \\
\hline Anteil qualifizierte Beschäftigte (in \%) & 1,019 & 8,55 & 0,000 \\
\hline Anteil weibliche Beschäftigte (in \%) & 0,087 & 0,68 & 0,494 \\
\hline Anteil Auszubildende (in \%) & 0,503 & 1,34 & 0,180 \\
\hline Betriebsgröße (Dummy)* & Referenzk & 100 bis & chäftigte \\
\hline 1 bis 4 Beschäftigte & $-1,627$ & $-11,74$ & 0,000 \\
\hline 5 bis 9 Beschäftigte & $-1,278$ & $-9,76$ & 0,000 \\
\hline 10 bis 19 Beschäftigte & $-1,041$ & $-7,84$ & 0,000 \\
\hline 20 bis 49 Beschäftigte & $-0,758$ & $-6,15$ & 0,000 \\
\hline 50 bis 99 Beschäftigte & $-0,326$ & $-2,35$ & 0,019 \\
\hline 200 bis 499 Beschäftigte & 0,198 & 1,27 & 0,205 \\
\hline 500 bis 999 Beschäftigte & 0,321 & 1,56 & 0,119 \\
\hline 1000 bis 4999 Beschäftigte & 1,072 & 2,80 & 0,005 \\
\hline Technische Anlagen auf neuem Stand (Dummy) & 0,198 & 3,01 & 0,003 \\
\hline Investitionen in Kommunikationstechnik (Dummy) & 0,371 & 5,80 & 0,000 \\
\hline Überstunden (Dummy) & 0,204 & 3,18 & 0,001 \\
\hline Fachkräftemangel (Dummy) & $-0,012$ & $-0,15$ & 0,881 \\
\hline Konstante & 0,395 & 1,51 & 0,132 \\
\hline $\begin{array}{lll}\text { * Betriebsgröße } 5000 \text { u. m. Beschäftigte aufgrund von } \\
\mathrm{N} & = & 2803 \\
\text { LR chi2(39) } & = & 1047,89 \\
\text { Prob > chi2 } & = & 0,0000 \\
\text { Log likelihood } & = & -1143,887 \\
\text { Pseudo R2 } & = & 0,3141\end{array}$ & learität aus & sen & \\
\hline
\end{tabular}

Quelle: LIAB-Längsschnitt, Wellen 1997 bis 2004, eigene Berechnungen 
Trotz der weitgehenden Übereinstimmung der Ergebnisse mit früheren Studien ist es wichtig, festzuhalten, dass das vorrangige Ziel der beschriebenen Probit-Schätzungen nicht die Ermittlung von Einflussparametern ist, sondern die eines balancing scores, der die oben beschriebene bedingte Unabhängigkeitsannahme glaubhaft macht und den Prozess der Selbstselektion in die Maßnahme „,betriebliche Weiterbildung“ abbildet. Zudem würde ohne das Vorhandensein der gefundenen systematischen Unterschiede in der Weiterbildungsaktivität der Common Support, der im Folgenden beschrieben wird, in sich zusammenfallen.

\section{4.2 Relevanz der Common-Support-Bedingung}

Nach diesem ersten Teil der Schätzung sollen nun - ausgehend von den errechneten propensity scores - die Effekte von Weiterbildung abgeschätzt werden. Wie oben beschrieben, müssen dabei die auszuwertenden Beobachtungen um diejenigen weiterbildenden Betriebe reduziert werden, für die keine geeigneten Vergleichsbetriebe aus der Kontrollgruppe gefunden werden konnten. Für die nachfolgenden Schätzungen wurde jeweils nur auf die um die Common-Support-Bedingung bereinigten Datensätze zurückgegriffen. Dabei blieben all jene Beobachtungen der Treatmentgruppe unberücksichtigt, für die ausgehend von der einleitenden Probit-Schätzung ein höherer Propensity-Score-Wert der Teilnahme errechnet wurde als der höchste Wert, der für die Kontrollgruppe ermittelt wurde. Im ersten Datensatz fielen damit 21 Betriebe aus der Analyse heraus, die in den Jahren 1993 bis 1995 kontinuierlich Weiterbildung finanziert hatten. Im zweiten Datensatz sind dies 325 der insgesamt 2028 weiterbildenden Betriebe. Die Darstellungen in Abb. 40 und Abb. 41, sowie Tab. 13 und Tab. 14 (alle im Anhang C) veranschaulichen die Analyse des Common Supports. In den beiden Grafiken sind jeweils die absoluten Häufigkeiten der Beobachtungen von Betrieben über Klassen des geschätzten propensity scores abgetragen. Die Höhe der Balken im positiven Bereich stellen die relativen Häufigkeiten der weiterbildenden Betriebe dar, die Balken im negativen Bereich die der Kontrollgruppe.

Ein Qualitätskriterium für die Beurteilung des Matching-Verfahrens ist der Mittelwertvergleich der Kovariaten für die Beobachtungen der Treatmentgruppe und der Kontrollgruppe: Nach Zuordnung von Beobachtungen der Kontrollgruppe ließen sich für die Schätzung der Effekte von durchgängiger Weiterbildung in den Jahren 1993 bis 1995 nur für die Variablen „Altersgruppe der 51- bis 55-Jährigen“ und die Branchendummies „Gesundheitswesen“ und „Freie Berufe u. ä.“ auf 5\% signifikante Unterschiede zwischen den beiden Vergleichsgruppen finden. Alle anderen Kovariaten wiesen keine statistisch abgesicherten Differenzen mehr auf. Die Mittelwertvergleiche für die Schätzungen des zweiten Datensatzes kommen zu ähnlichen Ergebnissen.

\section{4.3 Schätzungen des kausalen Effektes betrieblicher Weiterbildung}

Alle folgenden Berechnungen wurden mit Stata 8.2 mit dem ado-File ,psmatch2“ durchgeführt (vgl. Leuven und Sianesi (2003)). In den Tabellen Tab. und Tab. sind die Ergebnisse der ATT-Schätzungen mit Hilfe der beiden gewählten Matching-Algorithmen dargestellt. 
Dabei wird jeweils in der ersten Zeile ein einfacher Mittelwertvergleich zwischen den weiterbildenden Betrieben der Treatmentgruppe und der Kontrollgruppe dargestellt. Die folgenden beiden Zeilen zeigen die Ergebnisse des Matching: Zunächst wurde der Effekt nach der Nearest-neighbor-Zuordnung (NN) errechnet. Zum Vergleich werden danach die Ergebnisse verschiedener Spezifikationen der Bandbreite von Local-Linear-Matching-Schätzungen (LLR) dargestellt. Ihre Standardabweichungen der wurden ATT-Schätzungen durch ein Bootstrap mit 1000 Replikationen ermittelt. Die Verteilungseigenschaften der Nearestneighbor-Ergebnisse lassen sich dagegen nicht per Bootstrap ermitteln. Stattdessen sind hier die analytischen Standardabweichungen ausgewiesen, wohl wissend, dass dabei der Umstand nicht berücksichtigt wird, dass es sich bei den Werten des propensity scores selbst um geschätzte Werte handelt.

Tab. 20:

Matching-Ergebnisse: Finanzierung betrieblicher Weiterbildung 1993 bis 1995

\begin{tabular}{|c|c|c|c|c|c|c|c|}
\hline \multicolumn{2}{|c|}{ Anteil Mind.-50-Jähriger } & \multirow{2}{*}{$\begin{array}{c}\begin{array}{c}\text { Treatment- } \\
\text { gruppe }\end{array} \\
25,52 \%\end{array}$} & \multirow{2}{*}{$\begin{array}{c}\begin{array}{c}\text { Kontroll- } \\
\text { gruppe }\end{array} \\
26,99 \%\end{array}$} & \multirow{2}{*}{$\begin{array}{c}\text { Differenz } \\
-1,47 \%\end{array}$} & \multirow{2}{*}{$\frac{\text { Std.abw. }}{1,16 \%}$} & \multirow{2}{*}{$\frac{\text { t-Statistik }}{-1,26}$} & \multirow[t]{2}{*}{$P>|z|$} \\
\hline Methode & $\begin{array}{c}\text { Ohne } \\
\text { Matching }\end{array}$ & & & & & & \\
\hline NN & ATT & $25,99 \%$ & $28,54 \%$ & $-2,55 \%$ & $2,06 \%$ & $-1,24$ & 0,215 \\
\hline $\operatorname{LLR}(0,8)$ & ATT & $25,99 \%$ & $27,37 \%$ & $-1,38 \%$ & $1,19 \%$ & $-1,16$ & 0,247 \\
\hline $\operatorname{LLR}(0,6)$ & ATT & $25,99 \%$ & $26,73 \%$ & $-0,74 \%$ & $1,18 \%$ & $-0,63$ & 0,528 \\
\hline $\operatorname{LLR}(0,4)$ & ATT & $25,99 \%$ & $26,72 \%$ & $-0,73 \%$ & $1,19 \%$ & $-0,62$ & 0,537 \\
\hline $\operatorname{LLR}(0,2)$ & ATT & $25,99 \%$ & $26,73 \%$ & $-0,74 \%$ & $1,18 \%$ & $-0,63$ & 0,528 \\
\hline
\end{tabular}

Quelle: LIAB Längsschnitt, Wellen 1993, 1994, 1995 bis 2004, eigene Berechnungen

Danach lassen sich für den ersten Datensatz und die Schätzung von Auswirkungen durchgehender Weiterbildung in den Jahren 1993 bis 1995 auf den Anteil der Älteren im Betrieb 2004 keine Erhöhung des Anteils Älterer feststellen. Im Gegenteil ergibt sich für ein NearestNeighbor-Matching eine schwach signifikant negative Differenz, die auf einen durch die Weiterbildung verursachten Rückgang des Älterenanteils schließen lässt. Die LLR-Schätzungen kommen unabhängig von der Wahl der Bandbreit zu einem insignifikanten Ergebnis.

Tab. 21: Matching-Ergebnisse: Finanzierung betrieblicher Weiterbildung 1997

\begin{tabular}{|c|c|c|c|c|c|c|c|}
\hline \multicolumn{2}{|c|}{ Anteil Mind.-50-Jähriger } & \multirow{2}{*}{$\begin{array}{c}\begin{array}{c}\text { Treatment- } \\
\text { gruppe }\end{array} \\
27,44 \%\end{array}$} & \multirow{2}{*}{$\begin{array}{c}\begin{array}{c}\text { Kontroll- } \\
\text { gruppe }\end{array} \\
28,20 \%\end{array}$} & \multirow{2}{*}{$\begin{array}{c}\text { Differenz } \\
-0,76 \%\end{array}$} & \multirow{2}{*}{$\begin{array}{c}\text { Std.abw. } \\
0,83 \%\end{array}$} & \multirow{2}{*}{$\frac{\text { t-Statistik }}{-0,93}$} & \multirow[t]{2}{*}{$P>|z|$} \\
\hline Methode & $\begin{array}{c}\text { Ohne } \\
\text { Matching }\end{array}$ & & & & & & \\
\hline NN & ATT & $27,82 \%$ & $29,86 \%$ & $-2,03 \%$ & $2,52 \%$ & $-0,81$ & 0,418 \\
\hline $\operatorname{LLR}(0,8)$ & ATT & $27,82 \%$ & $30,07 \%$ & $-2,24 \%$ & $1,58 \%$ & $-1,42$ & 0,156 \\
\hline $\operatorname{LLR}(0,6)$ & ATT & $27,82 \%$ & $29,12 \%$ & $-1,30 \%$ & $1,31 \%$ & $-0,99$ & 0.320 \\
\hline $\operatorname{LLR}(0,4)$ & ATT & $27,82 \%$ & $29,18 \%$ & $-1,36 \%$ & $1,36 \%$ & -1.00 & 0,315 \\
\hline $\operatorname{LLR}(0,2)$ & ATT & $27,82 \%$ & $29,52 \%$ & $-1,70 \%$ & $1,45 \%$ & -1.17 & 0.241 \\
\hline
\end{tabular}

Quelle: LIAB Querschnitt, Wellen 1997 bis 2004, eigene Berechnungen

Für die Finanzierung im Jahr 1997 lässt sich sieben Jahre später ebenfalls kein positives Zeichen für den Verbleib Älterer im Betrieb erkennen: Im Falle der Finanzierung einer nicht spezifizierten Weiterbildungsaktivität erweisen sich die - ebenfalls negativen - Werte der 
Differenz als nicht signifikant. Die Ergebnisse für die einzelnen Arten von spezifischer Weiterbildung finden sich im Anhang C. Einzig die Finanzierung von externen Kursen, von der anzunehmen ist, dass für sie besonders restriktive betriebsinterne Zugangsvoraussetzungen gelten, wirkt auf die Altersstruktur, wiederum zuungunsten älterer Beschäftigter. Diese Aussage gilt allerdings nur für die Schätzung mittels des Nearest-Neighbor-Verfahrens. Für die anderen beiden, weniger selektiven Formen der Qualifizierung, interne Kurse und Weiterbildung am Arbeitsplatz, sind keine signifikanten Ergebnisse zu Tage getreten.

Die Schätzungen dämpfen damit die Erwartungen, dass betriebliche Weiterbildung die Arbeitsmarktaussichten Älterer verbessert. Zudem kommen die beiden asymptotisch identischen Schätzverfahren zu recht einheitlichen Ergebnissen und lassen diese robust gegenüber alternativen Spezifikationen des Matching-Algorithmus erscheinen. Ein Vergleich der Wirkungen unterschiedlicher Formen von Weiterbildung deutet an, dass eine altersselektive innerbetriebliche Allokation von Qualifizierungsmaßnahmen ${ }^{77}$ die Erwerbsaussichten der Älteren nicht nur unberührt lässt, sondern sogar verschlechtert und eine Verdrängung durch nun besser qualifizierte Jüngere nach sich zieht.

\section{5 Zusammenfassung: Wirkung betrieblicher Weiterbildung auf die Altersstruktur}

Die aufgrund ermutigender früherer Ergebnisse erhofften positiven Effekte betrieblicher Weiterbildung auf den Verbleib Älterer im Betrieb ließen sich hier nicht bestätigen. So konnten weder für verschiedene Zeiträume noch verschiedene Formen der Weiterbildung signifikante, positive Effekte festgestellt werden. Als Datengrundlage diente das IAB-Betriebspanel ergänzt um Angaben der Altersstruktur, die sich aus der Verknüpfung zum LIAB ergaben.

Zentrales methodisches Anliegen der Auswertung war die Kontrolle von Selbstselektion der Betriebe in eine Gruppe der Weiterbildenden und eine solche, die auf diese Maßnahme verzichtet. Dazu wurde ein Matching-Ansatz gewählt, der zunächst den Selektionsprozess schätzt und nach Zuordnung von weiterbildenden Betrieben zu solchen der Kontrollgruppe auf Basis dieser Schätzung die beiden Gruppen vergleicht. Die für das Verfahren notwendige Datenbasis scheint durch den großen Umfang an Kontrollvariablen mit dem IAB-Betriebspanel gegeben. Die beschriebenen Ergebnisse erweisen sich als robust gegenüber der Wahl des Matching-Algorithmus.

Aufgrund dieser Ergebnisse scheinen allgemeine Maßnahmen zur Förderung von betrieblicher Weiterbildung dann nicht angezeigt, wenn sie das Ziel verfolgen, die Beschäftigungschancen Älterer zu verbessern und die Lebensarbeitszeit zu verlängern. Stattdessen deuten die Resultate der Schätzungen auf einen, wenn auch nicht signifikanten, niedrigeren Anteil der 50-Jährigen und Älteren hin. Allerdings sei ausdrücklich darauf hingewiesen, dass in der vor-

\footnotetext{
${ }^{77} \mathrm{Zu}$ dem Ergebnis einer geringeren Beteiligung Älterer an betrieblicher Weiterbildung kommen beispielsweise die Erhebungen der CVTS (vgl. Statistisches Bundesamt (2007), S. 30).
} 
liegenden Studie nicht die Wirkungen individueller Weiterbildung analysiert wurden, sondern die Weiterbildungsaktivität von Betrieben generell. Auswertungen von statistischen Erhebungen zum individuellen Zugang zu Weiterbildung und ihren - auch altersspezifischen - Wirkungen legen den Schluss nahe, dass eine altersselektive Zuteilung zu Verdrängungseffekten führt, die einer politisch gewünschten Verlängerung der Erwerbsbeteiligung im Wege steht. Statt Weiterbildung als Allheilmittel allgemein zu fördern, sollten daher Anreize geschaffen werden, um auch ältere Beschäftigtengruppen an diesen Maßnahmen stärker zu beteiligen. 


\section{Zusammenfassung und Ausblick}

Der Arbeitsmarkt für Ältere rückt mit seiner Bedeutung für die Bewältigung der Probleme des demografischen Wandels in den Mittelpunkt des öffentlichen Interesses. Wie gelingt es, Ältere länger als bisher in Beschäftigung zu halten? Wie können ihre Wiedereinstellungschancen verbessert werden? Zur Beantwortung dieser Fragen soll diese Arbeit Anhaltspunkte geben.

Die vergangenen Jahre zeigten eine Verbesserung der Arbeitsmarktintegration Älterer, die die geänderte Zielrichtung der Arbeitsmarkt- und Sozialpolitik reflektiert. Herrschte bis in die frühen 90er Jahre die Ansicht vor, mit Hilfe von Frühverrentungen und anderen, den Arbeitsmarkt entlastenden Maßnahmen die Eingliederung Jüngerer zu begünstigen, traten danach Sorgen um die Finanzierbarkeit der Sozialversicherungen in den Vordergrund, denen nun mit einer Verlängerung der Lebensarbeitszeit begegnet werden sollte. Dennoch ist die Langzeitarbeitslosigkeit unter Älteren in Deutschland immer noch hoch und deutet darauf hin, dass weiterhin Defizite in der Integration arbeitswilliger Älterer bestehen.

Anders als viele andere untersuchen die hier vorgestellten Arbeiten daher auch die Nachfrageseite des Arbeitsmarktes. Den Einzeluntersuchungen ist gemein, dass sie sich auf eine Kombination von Individual- und Betriebsinformationen stützen, die mit mikroökonometrischen Methoden ausgewertet werden. Ermöglicht wurden diese Analysen durch den verbesserten Zugang der Wissenschaft zu Individualdaten durch die Produzenten, in diesem Fall der BA, und der Bereitstellung neuer Datensätze, in diesem Fall des LIAB. Erst hierdurch ließen sich betriebliches Verhalten wie auch individuelle (Erwerbs-) Lebensverläufe in ihrem Zusammenspiel untersuchen.

Kapitel A untersucht, wie Betriebe das Erwerbsaustrittsverhalten ihrer älteren Arbeitnehmer beeinflussen. In Hazardratenmodellen kommt ein spezieller LIAB-Datensatz der Jahre 1996 bis 2004 zum Einsatz, um Bestimmungsgründe für das Ende des individuellen Arbeitslebens zu ermitteln. Dabei kommt zum Vorschein, dass Institutionen wie der Kündigungsschutz, Mitbestimmung und Tarifbindung keine beschäftigungsverlängernde Wirkung entfalten. Es zeigt sich dagegen, dass sie die Position Älterer im Betrieb untergraben und einen frühen Austritt begünstigen. Die Annahme, dass die betriebliche Entscheidung über die Weiterbeschäftigung Älterer in starkem Ausmaß als Anpassungsinstrument des Personalbedarfs bei Nachfrageschwankungen dient, konnte allgemein bestätigt werden. Die Abhängigkeit der Austrittswahrscheinlichkeit von den Umsatzerwartungen des beschäftigenden Betriebes spricht hier eine deutliche Sprache. Andererseits ließen sich auch Anzeichen für Bedingungen finden, unter denen die Generation der Über-50-Jährigen dem Betrieb, dem Arbeitsmarkt und schließlich den Sozialversicherten als Beitragszahler erhalten bleiben kann. Es zeigt sich, dass ein hoher Anteil Jüngerer im Betrieb die Austrittswahrscheinlichkeit Älterer senkt. Der Furcht vor dem technischen Wandel im Zusammenhang mit dem Ziel einer Erhöhung der Erwerbsbeteiligung Älterer konnte hier keine Nahrung gegeben werden. Die Ergebnisse deuten im 
Gegenteil darauf hin, dass eine moderne technische Ausstattung des Betriebes sich positiv auf die Erwerbsaussichten auch der Älteren auswirkt. Genauso wenig ließen sich nachteilige, kurzfristige Effekte der Einführung neuer Technik nachgewiesen.

Mit den Ergebnissen bestätigt sich die Erwartung, dass beim Übergang aus dem Erwerbs- in die Ruhephase der beschäftigende Betrieb und damit die Nachfrageseite des Arbeitsmarktes eine wichtige Rolle spielen.

Eine spezielle Auseinandersetzung widmet Kapitel B dem Kündigungsschutz und seinem Schwellenwert. Es gibt gute Gründe für die These, dass insbesondere Ältere ihre Wiederbeschäftigungschancen durch das geltende Kündigungsschutzrecht und seinen Schwellenwert berechtigt geschmälert sehen. Insbesondere die Sozialauswahl mit ihrer vermeintlichen Schutzfunktion für die ältere Belegschaft stehe einer Neueinstellung Älterer im Wege, so die landläufige Meinung. Diese Vermutung lässt sich durch die Untersuchung der LIAB-Daten nicht bestätigen. Angesichts der verwendeten RDD-Methode, die Gesetzesänderungen als quasi-natürliche Experimente nutzt, erweist sich der empirische Ansatz als unabhängig von Fragen der notwendigen Kontrolle von Einflussmerkmalen und der geeigneten parametrischen Form.

Genauso wenig ließ sich die Hoffnung bestätigen, dass sich Betriebe durch Weiterbildung allein zu einem verstärkten Einsatz Älterer bewegen lassen. Die in Kapitel C geschätzten Effekte von Weiterbildung auf den Anteil Älterer waren bestenfalls insignifikant. Wieder gingen kombinierte Angaben aus IAB-Betriebspanel und der Beschäftigtenstatistik in die Auswertungen ein. Der möglichen Endogenität der betrieblichen Weiterbildungsentscheidung wurde mittels eines Matching-Verfahrens Rechnung getragen. Der vermutete kausale Effekt betrieblicher Weiterbildung hin zu einem höheren Anteil Älterer konnte in einem Vergleich der Betriebspaare nicht nachgewiesen werden. Hier könnte eine altersselektive Auswahl jüngerer Teilnehmer zu einem Hinausdrängen derjenigen geführt haben, die eben nicht von der Weiterbildungsaktivität ihres Arbeitgebers profitieren.

Aufgrund der Größenstruktur und Branchenzugehörigkeit von freiberuflichen Betrieben lassen sich aus den Ergebnissen auch Schlüsse hinsichtlich der Rolle der Freien Berufe als Arbeitgeber. Danach bieten Arbeitgeber des Dienstleistungssektors Älteren Arbeitsplätze, die eine lange Erwerbstätigkeit versprechen und einem geringen Risiko des frühzeitigen Austritts ausgesetzt sind. Die Kontrolle für Kleinstbetriebe relativierte die Aussagen, dadurch dass sie Älteren tendenziell schlechtere Jobaussichten bieten als größere Betriebe. Auch die Ergebnisse zu den geringen nachweisbaren Wirkungen des Kündigungsschutzes auf die Einstellung von Älteren lassen sich auf die Freiberufler übertragen. Eine explizite Berücksichtigung der Freiberufler war angesichts der Datenlage nicht möglich. Ebenso können die Wirkungen von Weiterbildung aus dem Blickwinkel der Freien Berufe betrachtet werden. Auch wenn die Studie in Kapitel C den Kleinbetrieben im Allgemeinen eine geringere Weiterbildungsaktivität attestiert, muss dies, wie die Arbeit zeigt, nicht zum Nachteil der Älteren sein. 
Keine der einzelnen Untersuchungen enthält ein Heilsversprechen, sondern zeigt eher auf, welche möglichen Wege nicht zum Ziel führen. Dennoch lassen sich einzelne Teilergebnisse zu positiven Aussagen zusammenfügen. So zeigt sich im ersten Teil, dass Unternehmen Möglichkeiten haben, ihre Beschäftigten als solche zu behalten. Altersgemischte Gruppen scheinen sich förderlich auf die Integration der Älteren auszuwirken. Auch müssen Betriebe und arbeitsmarktpolitische Akteure keine falsche Vorsicht bei der Einführung neuer Technik walten lassen. Im Mittel wirken solche Änderungen eben nicht nachteilig, sondern langfristig sogar vorteilhaft in Hinblick auf den Verbleib Älterer.

Die Frage bleibt, welchen Anreiz Betriebe haben, geeignete Maßnahmen zu ergreifen. Hier könnten die erwähnten Änderungen im Rentenzugangsrecht ins Spiel kommen. Die hierdurch verlängerte Erwerbsperspektive könnte dazu führen, dass sowohl Arbeitnehmer wie -geber Maßnahmen ergreifen, um auch im einem Alter, in dem früher der schleichende Übergang in den Ruhestand geplant wurde, die eigene Beschäftigungsfähigkeit und die der Angestellten zu erhalten. So könnte sich möglicherweise mit der Anhebung des Zugangsalters - und damit der Verlängerung der Amortisationszeit der Investition - die Benachteiligung von Älteren beim Zugang zu Weiterbildung verringern.

Für zukünftige Untersuchungen der Übergangsprozesse am Ende des Erwerbslebens wäre es von Vorteil, die vorhandenen Daten weiter aufzubereiten. Für die Finanzierung der Sozialversicherungen ist neben dem Ende der individuellen Beitragszahlungen insbesondere der Renteneintritt und damit der Beginn von Auszahlungen von besonderer Bedeutung. Es wäre daher wünschenswert, wenn Information über einen definitiven Rentenzugang und die Art des Zugangs in einer Datengrundlage integriert würde. Auch eine genauere Erfassung von Anwartschaften und dem Versicherungsverlauf, die für den Bezug von vorzeitigen Altersrenten entscheidend sind, würde zukünftigen Auswertungen zugute kommen. Auch haben frühere Studien gezeigt, dass mit der Einbeziehung von Variablen des familiären Kontexts, wie Angaben zu Kindern und insbesondere Ehepartner darstellen, die Modellierung der Ruhestandsentscheidung verbessern lässt. Am Ende könnte das Ziel lauten, einen gemeinsamen, integrierten Datenbestand aller Sozialversicherer aufzubauen, der die einzeln vorliegenden Datensätze vereint und kein zusätzliches Risiko der Deanonymisierung von Individuen mit sich bringt. Nur dann könnten sich Erweiterungen der hier vorgestellten Arbeiten durchführen lassen, wie beispielsweise den Übergang zwischen Erwerbs- und Ruhestandsphase in seinen unterschiedlichen Verlaufsformen zu untersuchen. 


\section{Anhang A}

Tab. 12: Durchschnittsalter des Austritts aus der Erwerbstätigkeit nach Altersgruppen im Jahr 1996 und Personenvariablen

\begin{tabular}{|c|c|c|c|c|c|c|c|c|c|c|}
\hline \multirow[b]{2}{*}{ Variable } & \multicolumn{2}{|c|}{ Alle } & \multicolumn{2}{|c|}{$50-55$} & \multicolumn{2}{|c|}{$56-60$} & \multicolumn{2}{|c|}{$61-65$} & \multicolumn{2}{|c|}{$>65$} \\
\hline & Alter & n & Alter & $\mathbf{n}$ & Alter & $\mathbf{n}$ & Alter & $\mathbf{n}$ & Alter & $\mathbf{n}$ \\
\hline Alle & 59,9 & 487662 & 58,6 & 296865 & 61,1 & 163815 & 64,9 & 20002 & 72,8 & 6980 \\
\hline \multicolumn{11}{|l|}{ Geschlecht } \\
\hline Mann & 60,1 & 298636 & 58,6 & 175175 & 61,5 & 104859 & 64,8 & 14728 & 72,7 & 3874 \\
\hline Frau & 59,5 & 189026 & 58,5 & 12690 & 60,4 & 58956 & 65,3 & 5274 & 72,9 & 3106 \\
\hline \multicolumn{11}{|l|}{ Nationalität } \\
\hline Deutsch & 59,9 & 461424 & 58,6 & 279630 & 61,1 & 156307 & 64,9 & 18679 & 72,8 & 6808 \\
\hline Nicht deutsch & 59,4 & 26238 & 57,9 & 17235 & 61,5 & 7508 & 64,8 & 1323 & 71,6 & 172 \\
\hline \multicolumn{11}{|l|}{ Region } \\
\hline Ost & 59,3 & 188130 & 58,3 & 122194 & 60,3 & 58313 & 65,0 & 5916 & 71,7 & 1707 \\
\hline West & 60,3 & 299531 & 58,7 & 174671 & 61,6 & 105501 & 64,9 & 14086 & 73,1 & 5273 \\
\hline \multicolumn{11}{|l|}{$\begin{array}{l}\text { Ausbildung } \\
\text { bis mittlere Reife ohne }\end{array}$} \\
\hline $\begin{array}{l}\text { bis mittlere Reife ohne } \\
\text { Berufsausbildung } \\
\text { Bis mittlere Reife mit }\end{array}$ & 59,8 & 84444 & 58,4 & 51063 & 60,9 & 28554 & 64,9 & 3398 & 73,0 & 1429 \\
\hline $\begin{array}{l}\text { Berufsausbildung } \\
\text { (Fach)Hochschulreife }\end{array}$ & 59,6 & 290791 & 58,6 & 186292 & 60,9 & 94026 & 64,6 & 8370 & 72,9 & 2103 \\
\hline $\begin{array}{l}\text { ohne Berufsausbildung } \\
\text { (Fach)Hochschulreife mit }\end{array}$ & 60,7 & 1836 & 58,8 & 953 & 62,1 & 718 & 65,0 & 145 & 72,8 & 20 \\
\hline Berufsausbildung & 60,1 & 9287 & 58,7 & 5346 & 61,3 & 3437 & 64,7 & 402 & 72,9 & 102 \\
\hline Fachhochschulabschluss & 60,0 & 21793 & 58,6 & 12463 & 61,5 & 8315 & 64,4 & 953 & 72,8 & 62 \\
\hline Hochschulabschluss & 61,3 & 25910 & 59,1 & 11319 & 62,4 & 11844 & 64,8 & 2552 & 71,1 & 195 \\
\hline fehlt/keine Angabe & 61,0 & 52971 & 58,5 & 29184 & 61,9 & 16602 & 65,9 & 4120 & 72,7 & 3065 \\
\hline \multicolumn{11}{|l|}{ Berufliche Stellung } \\
\hline Auszubildende & 60,4 & 149 & 58,6 & 54 & 60,1 & 79 & 64,1 & 10 & 74,5 & 6 \\
\hline einfache Arbeiter & 59,1 & 81497 & 58,0 & 52329 & 60,6 & 26344 & 64,3 & 2377 & 72,6 & 429 \\
\hline Facharbeiter & 58,9 & 72776 & 58,0 & 47682 & 60,4 & 23322 & 63,9 & 1656 & 71,3 & 116 \\
\hline Meister u.a. & 59,7 & 8938 & 58,3 & 5089 & 61,1 & 3417 & 63,9 & 412 & 75,1 & 20 \\
\hline Angestellte & 59,8 & 165646 & 58,3 & 92489 & 61,2 & 65088 & 64,3 & 7684 & 72,4 & 385 \\
\hline Teilzeit<15h & 63,1 & 54584 & 60,1 & 23519 & 63,1 & 19313 & 66,1 & 6461 & 72,8 & 5291 \\
\hline Teilzeit>15h & 59,7 & 102948 & 59,1 & 75199 & 60,7 & 25822 & 65,6 & 1291 & 73,2 & 636 \\
\hline
\end{tabular}

Quelle: LIAB, eigene Berechnungen 
Abb. 28: Kaplan-Meier-Schätzung nach Geschlecht

Kaplan-Meier: Überleben in sv-pflichtiger Beschäftigung nach Geschlecht

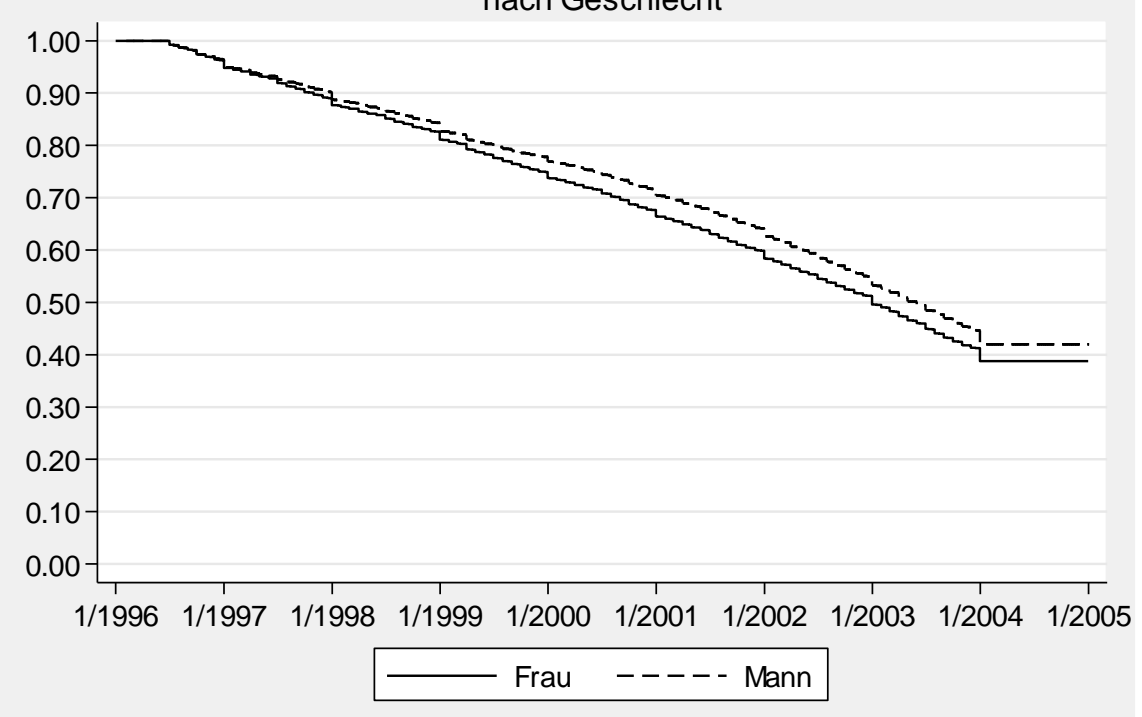

Quelle: LIAB, eigene Berechnungen

Abb. 29: Kaplan-Meier-Schätzungen nach Region

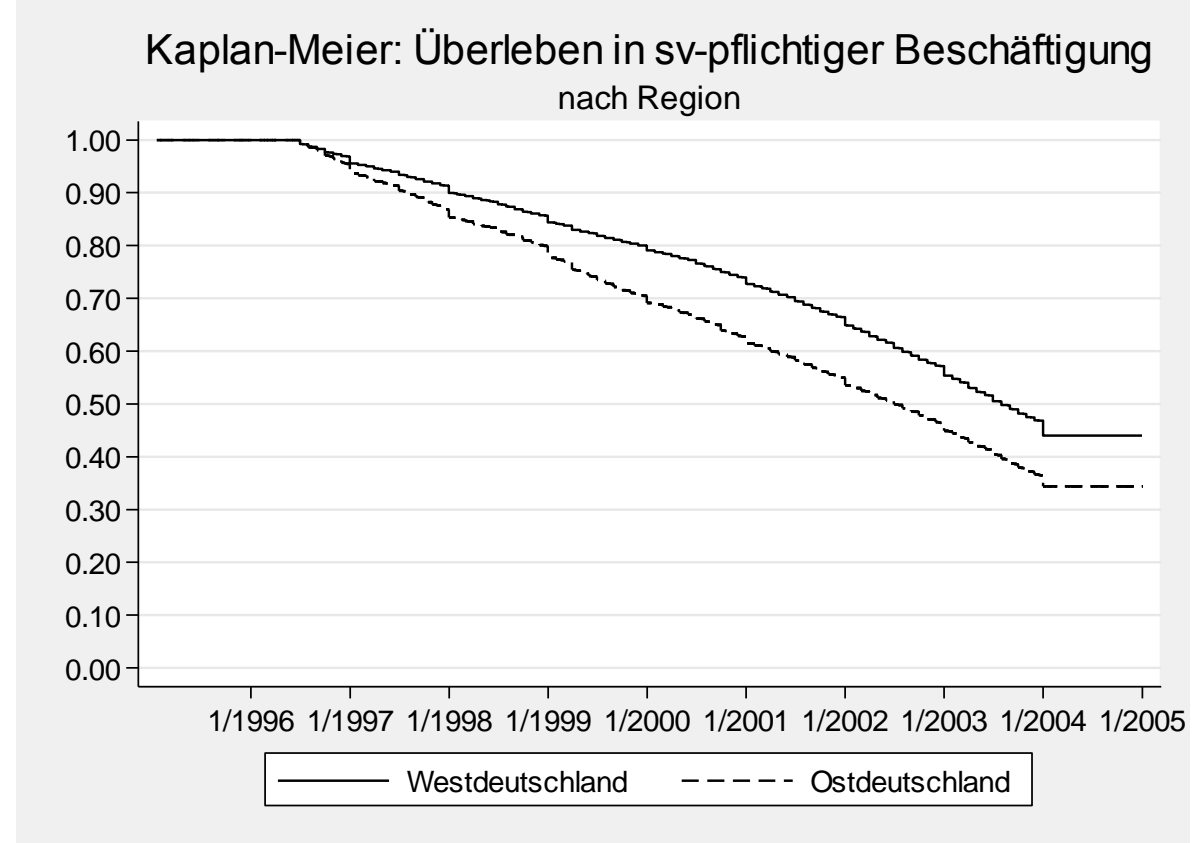

Quelle: LIAB, eigene Berechnungen 
Abb. 30:

Kaplan-Meier-Schätzungen nach Ausbildung

Kaplan-Meier: Überleben in sv-pflichtiger Beschäftigung nach Ausbildung

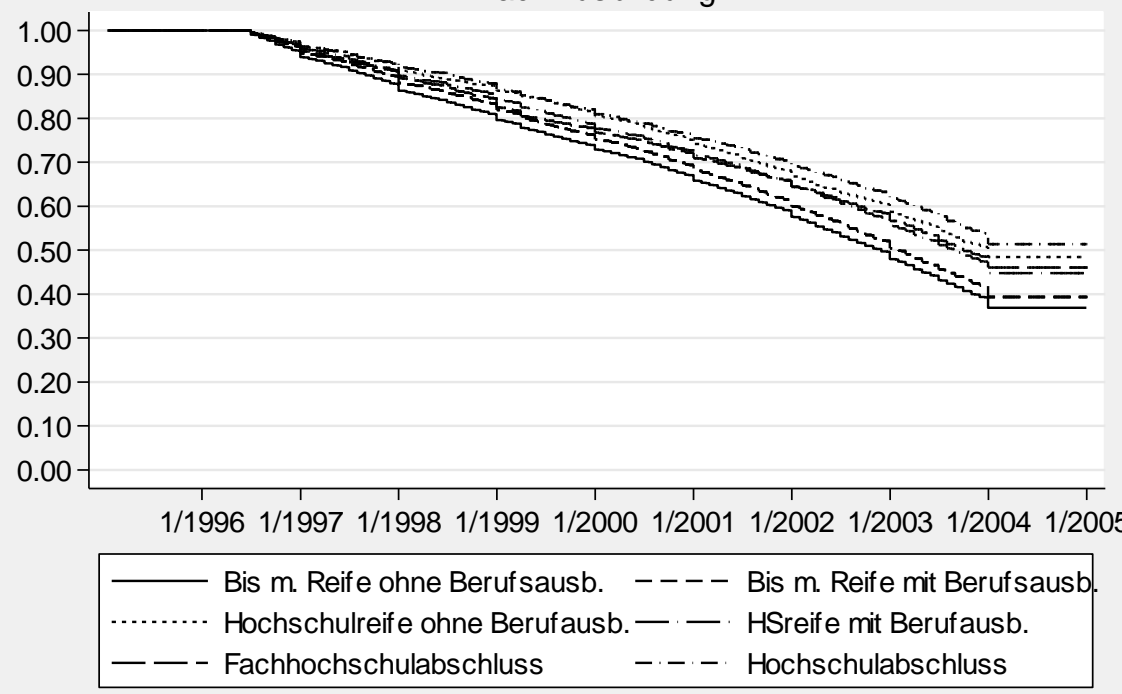

Quelle: LIAB, eigene Berechnungen

Abb. 31: Kaplan-Meier-Schätzung nach beruflicher Stellung

Kaplan-Meier: Überleben in sv-pflichtiger Beschäftigung nach beruflicher Stellung

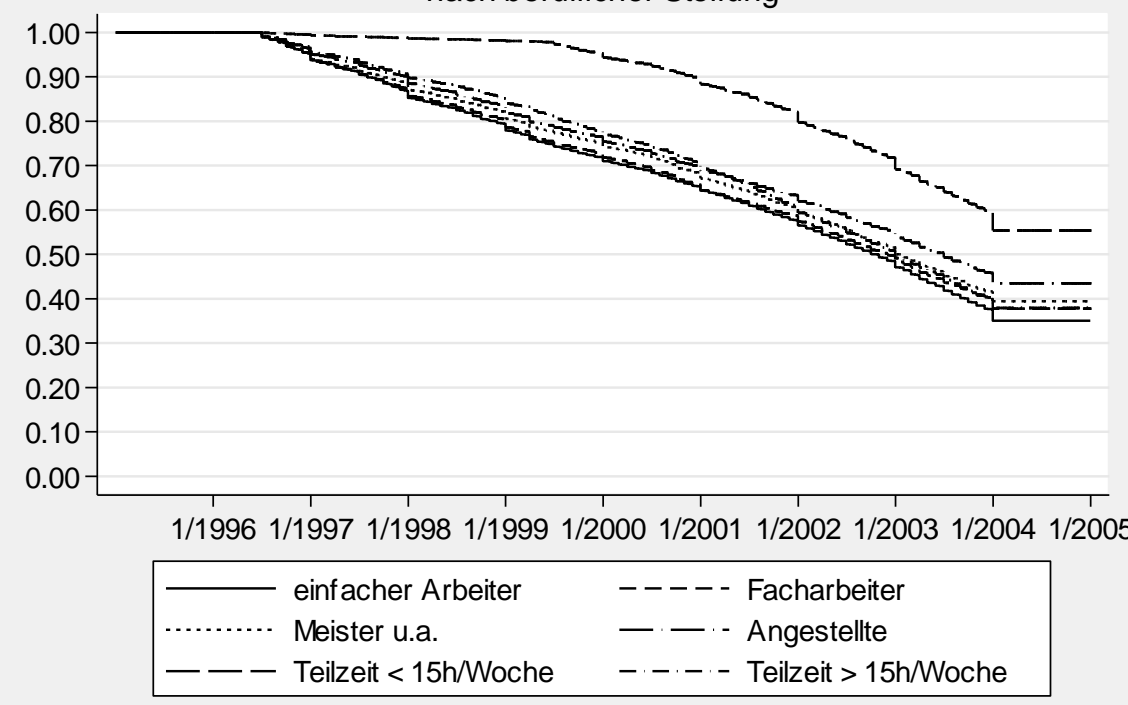

Quelle: LIAB, eigene Berechnungen 
Abb. 32: Kaplan-Meier-Schätzung nach Eintritt in den Betrieb

Kaplan-Meier: Überleben in sv-pflichtiger Beschäftigung nach Eintritt in den Betrieb

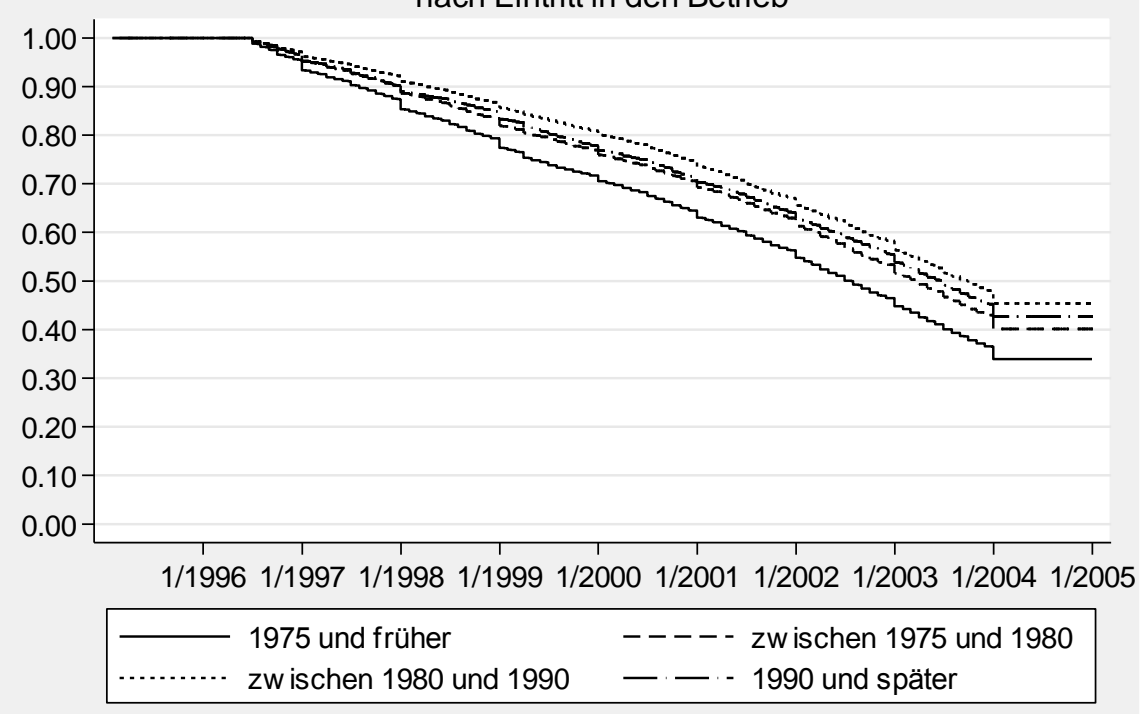

Quelle: LIAB, eigene Berechnungen

Abb. 33: Kaplan-Meier-Schätzung nach Eintritt in das Erwerbsleben

Kaplan-Meier: Überleben in sv-pflichtiger Beschäftigung nach Eintritt in das Erwerbsleben

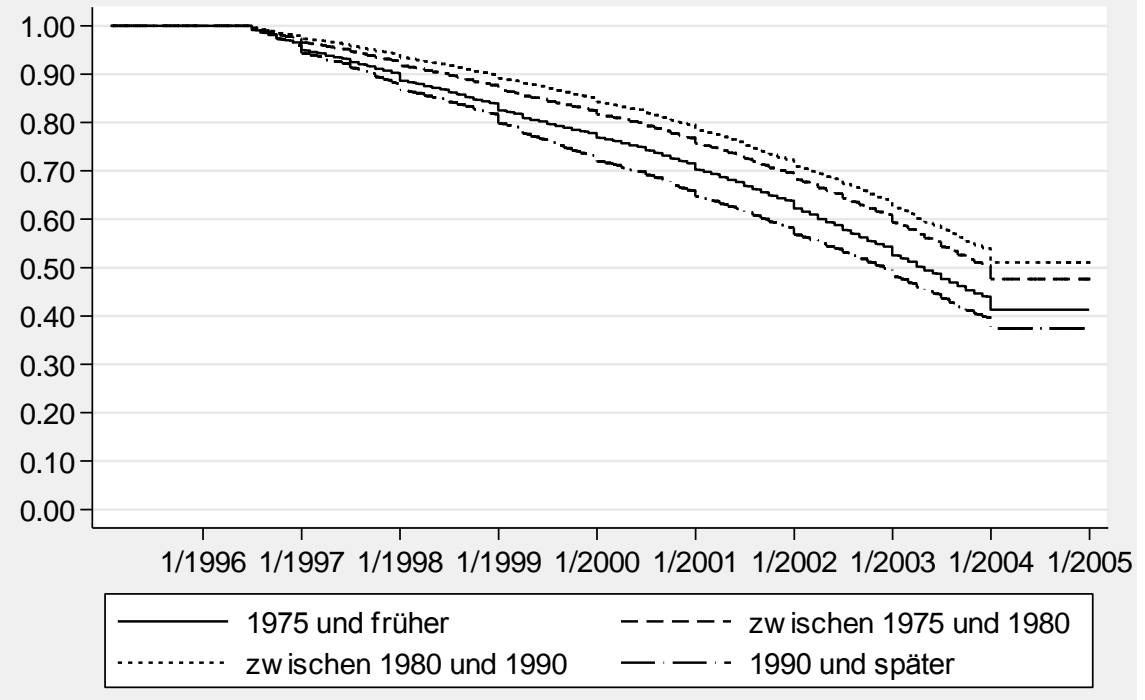

Quelle: LIAB, eigene Berechnungen 
Abb. 34: Kaplan-Meier-Schätzung nach Klassen von Tagesentgelt

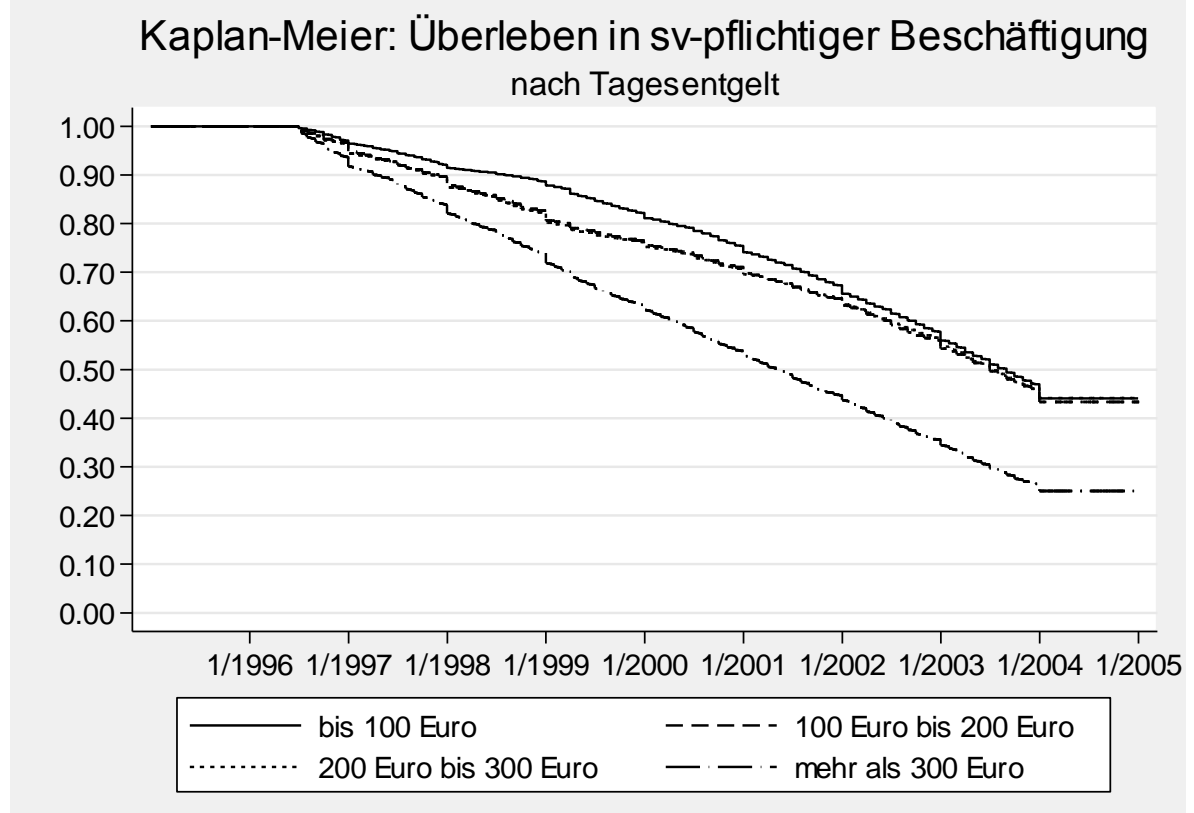

Quelle: LIAB, eigene Berechnungen

Abb. 35: Kaplan-Meier-Schätzungen nach Freistellung in den vergangenen zwei Jahren

Kaplan-Meier: Überleben in sv-pflichtiger Beschäftigung nach Freistellung letzte zwei Jahre

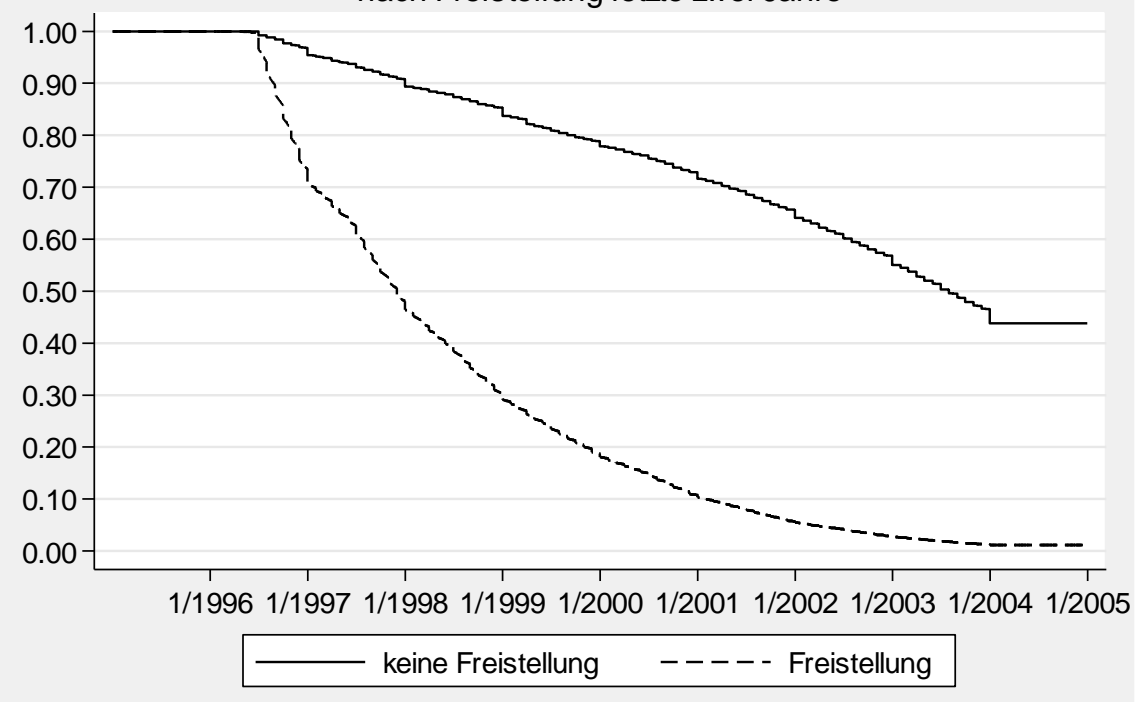

Quelle: LIAB, eigene Berechnungen 


\section{Anhang B}

Abb. 36: Schätzungen des LATE nach Bandbreite
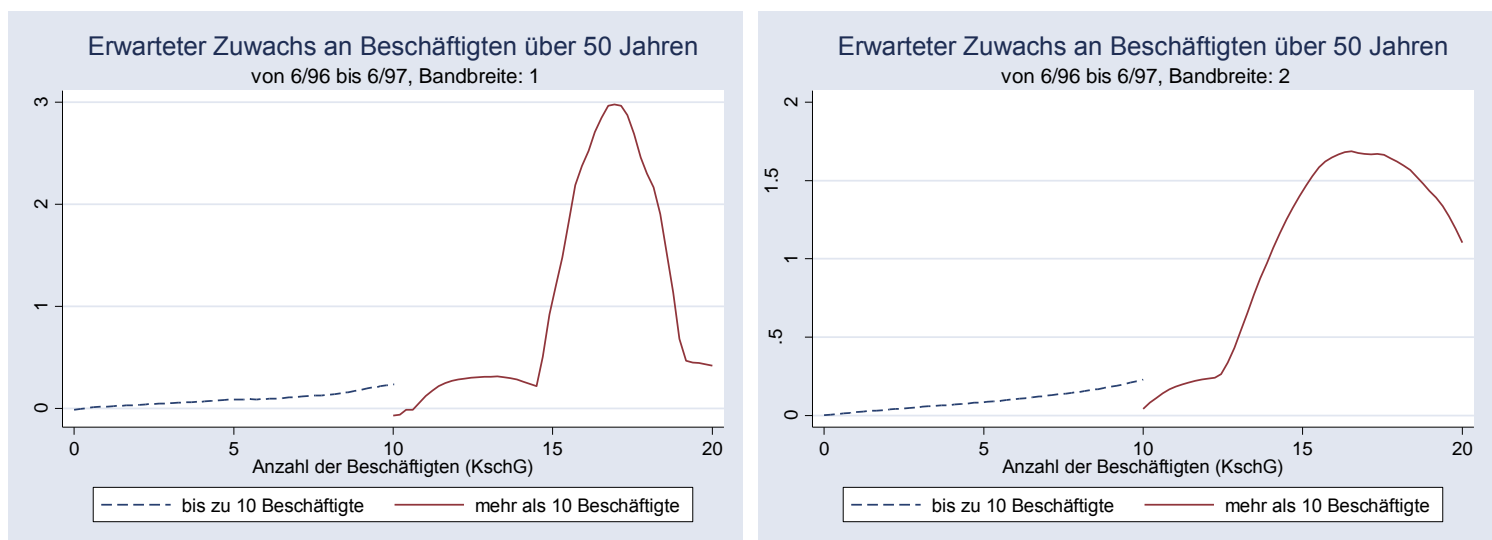

Erwarteter Zuwachs an Beschäftigten über 50 Jahren von $6 / 96$ bis $6 / 97$, Bandbreite: 3

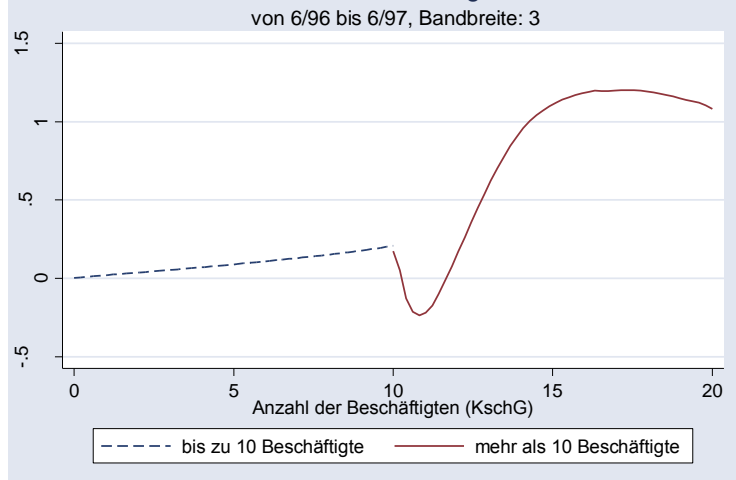

Erwarteter Zuwachs an Beschäftigten über 50 Jahren von $6 / 96$ bis $6 / 97$, Bandbreite: 4

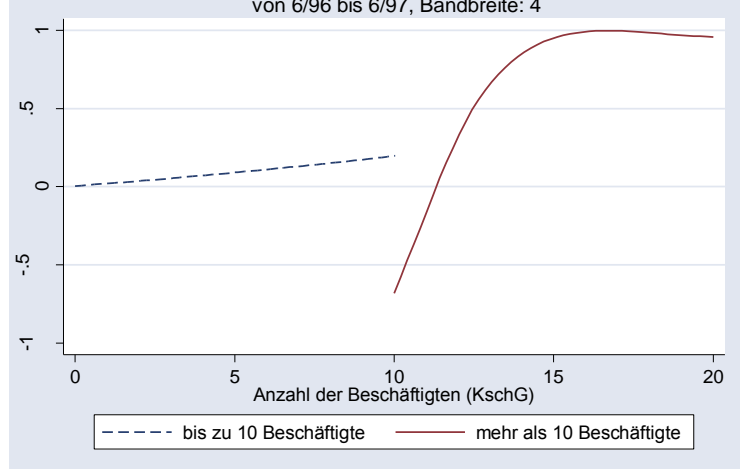

Erwarteter Zuwachs an Beschäftigten über 50 Jahren von $6 / 96$ bis $6 / 97$, Bandbreite: 5

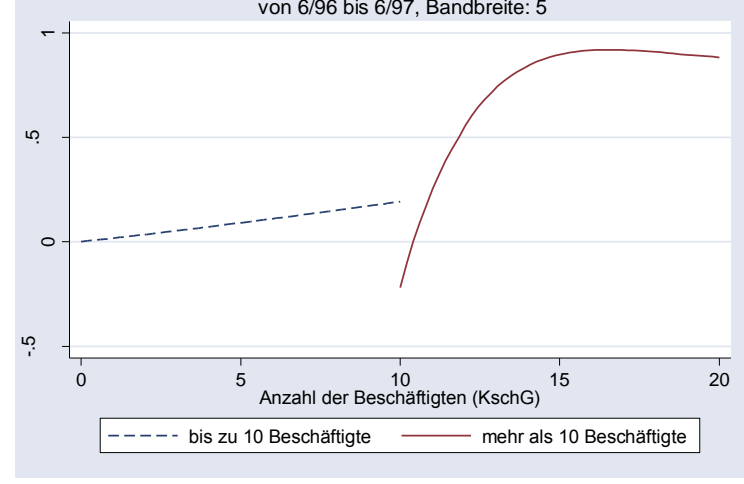

Quelle: IAB-Betriebspanel, eigene Berechnungen 


\section{Anhang C}

Abb. 37: $\quad$ Betriebliche Altersstruktur 2004 nach externen Kursen 1997

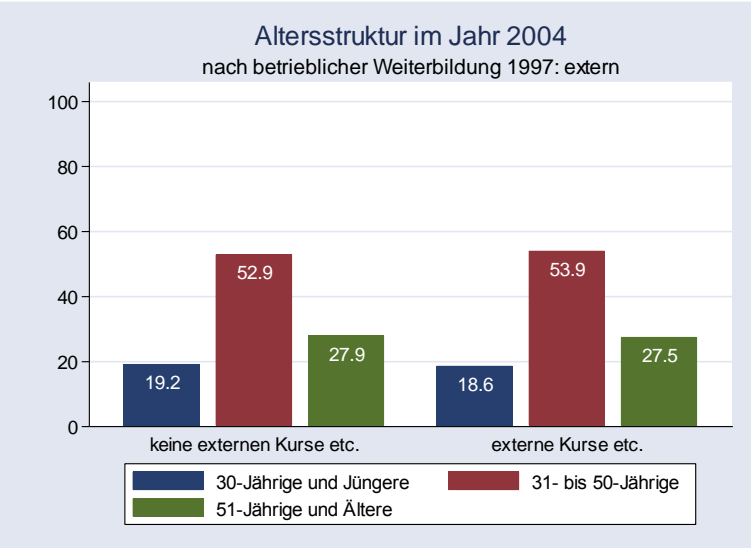

Quelle: LIAB-Querschnitt Wellen 1997 - 2004

Abb. 38: $\quad$ Betriebliche Altersstruktur 2004 nach internen Kursen 1997

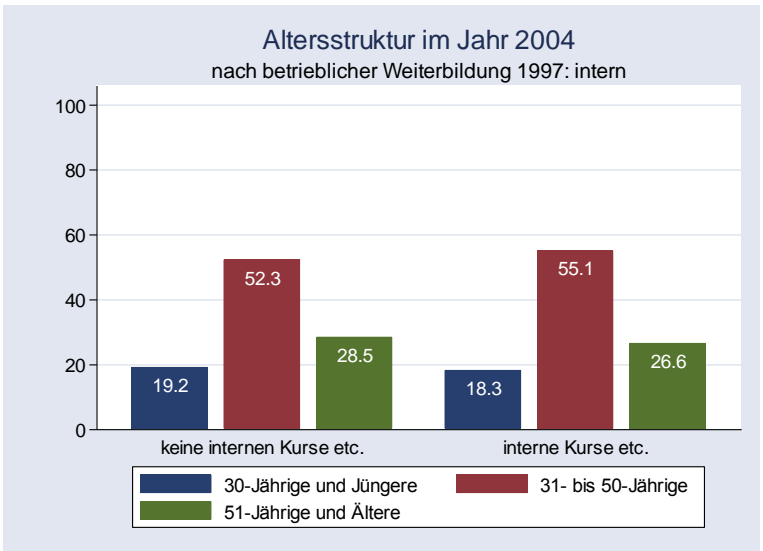

Quelle: LIAB-Querschnitt Wellen 1997 - 2004

Abb. 39: $\quad$ Betriebliche Altersstruktur 2004 nach WB am Arbeitsplatz 1997

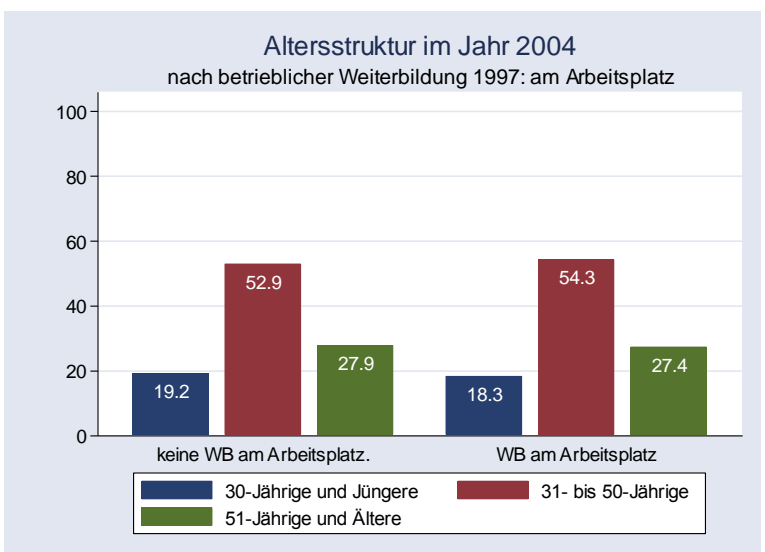

Quelle: LIAB-Querschnitt Wellen 1997 - 2004 
Abb. 40: Common Support ATT-Schätzung durchg. betrieblicher Weiterbildung 1993 bis 1995

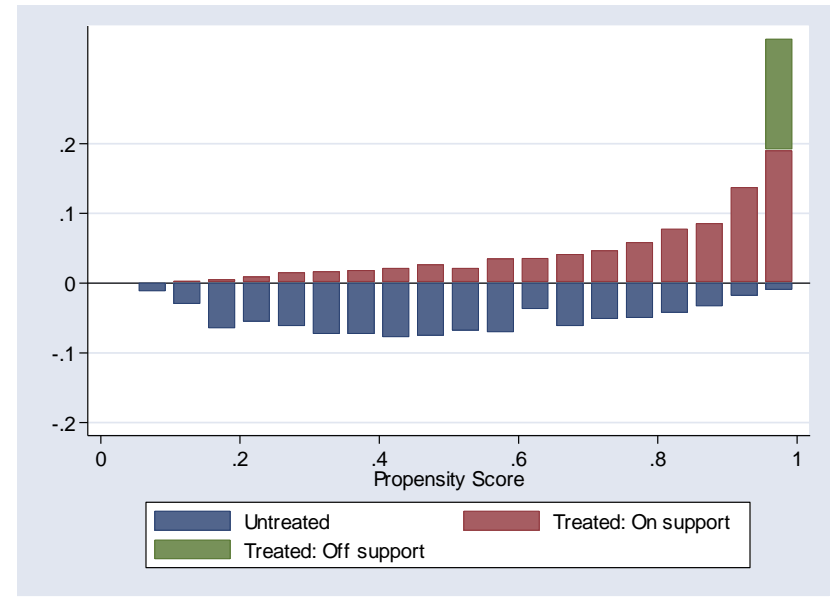

Quelle: IAB-Betriebspanel, Wellen 1993, 1994 und 1995, eigene Berechnungen

Tab. 13: $\quad$ Common Support ATT-Schätzung durchg. betrieblicher Weiterbildung 1993 bis 1995

\begin{tabular}{lccc}
\hline & \multicolumn{2}{c}{ COMMON SUPPORT } \\
& Außerhalb & Innerhalb & Gesamt \\
\hline \hline Keine Weiterbildung & 0 & 380 & 380 \\
Weiterbildung & 21 & 378 & 399 \\
Gesamt & 21 & 758 & 779 \\
\hline
\end{tabular}

Quelle: IAB-Betriebspanel, Wellen 1993, 1994 und 1995, eigene Berechnungen

Abb. 41: $\quad$ Common Support für die ATT-Schätzung betrieblicher Weiterbildung 1997

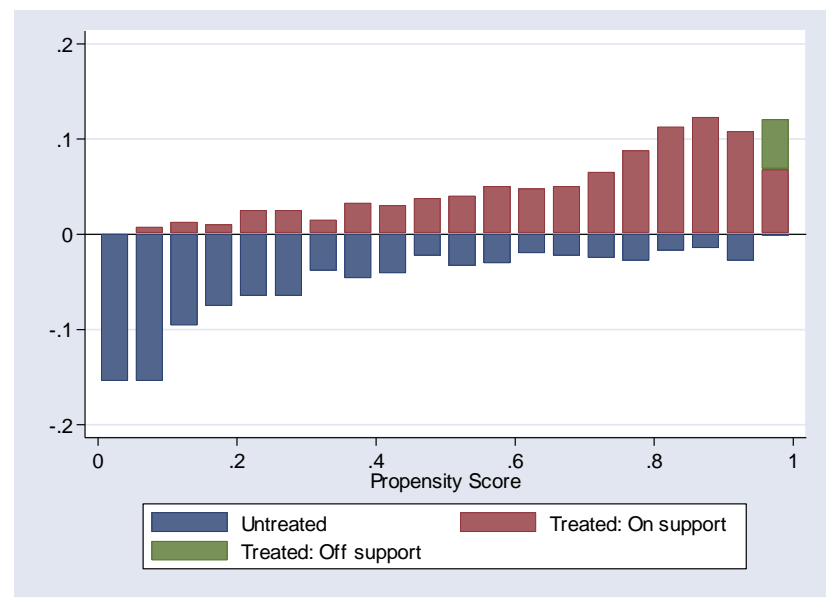

Quelle: IAB-Betriebspanel, Wellen 1997, eigene Berechnungen

Tab. 14: Common Support für die ATT-Schätzung betrieblicher Weiterbildung 1997

\begin{tabular}{lccc}
\hline & \multicolumn{2}{c}{ COMMON SUPPORT } \\
& Außerhalb & Innerhalb & Gesamt \\
\hline \hline Keine Weiterbildung & 0 & 791 & 791 \\
Weiterbildung & 325 & 1703 & 2028 \\
Gesamt & 325 & 2494 & 2819 \\
\hline
\end{tabular}

Quelle: IAB-Betriebspanel, Wellen 1997, eigene Berechnungen 
Tab. 15: Probit-Schätzung der Finanzierung von externen Kursen

\begin{tabular}{|c|c|c|c|c|}
\hline \multicolumn{2}{|c|}{ Weiterbildung: externe Kurse } & Koeffizient & $\mathbf{Z}$ & $P>|z|$ \\
\hline \multicolumn{2}{|c|}{ Altersstruktur 1997 (in \%) } & \multicolumn{3}{|c|}{ Referenzkategorie: Anteil bis 25-Jährige } \\
\hline \multicolumn{2}{|c|}{ Anteil 26- bis 30-Jährige } & $-0,348$ & $-1,30$ & 0,193 \\
\hline \multicolumn{2}{|c|}{ Anteil 31- bis 35-Jährige } & $-0,384$ & $-1,52$ & 0,128 \\
\hline \multicolumn{2}{|c|}{ Anteil 36- bis 40-Jährige } & $-0,530$ & $-2,02$ & 0,043 \\
\hline \multicolumn{2}{|c|}{ Anteil 41- bis 45-Jährige } & $-0,531$ & $-1,91$ & 0,056 \\
\hline \multicolumn{2}{|c|}{ Anteil 46- bis 50 -Jährige } & $-0,422$ & $-1,58$ & 0,115 \\
\hline \multicolumn{2}{|c|}{ Anteil 51- bis 55-Jährige } & $-0,143$ & $-0,44$ & 0,657 \\
\hline \multicolumn{2}{|c|}{ Anteil 56- bis 60 -Jährige } & $-0,482$ & $-1,37$ & 0,170 \\
\hline \multicolumn{2}{|c|}{ Anteil 61- bis 65-Jährige } & $-0,593$ & $-0,65$ & 0,516 \\
\hline \multicolumn{2}{|c|}{ Anteil über 65-Jährige } & $-0,111$ & $-0,06$ & 0,949 \\
\hline \multicolumn{2}{|c|}{ Branche (Dummy) } & \multicolumn{3}{|c|}{ Referenzkategorie: Land- und Forstwirtschaft } \\
\hline \multicolumn{2}{|c|}{ Energie und Bergbau } & 2,040 & 4,15 & 0,000 \\
\hline \multicolumn{2}{|c|}{ Grundstoffverarbeitung } & 0,606 & 3,19 & 0,001 \\
\hline \multicolumn{2}{|c|}{ Investitionsgüter } & 0,710 & 4,16 & 0,000 \\
\hline \multicolumn{2}{|c|}{ Verbrauchsgüter } & 0,296 & 1,65 & 0,100 \\
\hline \multicolumn{2}{|c|}{ Baugewerbe } & 0,332 & 1,94 & 0,053 \\
\hline \multicolumn{2}{|c|}{ Handel } & 0,303 & 1,81 & 0,071 \\
\hline \multicolumn{2}{|c|}{ Verkehr und Nachrichten } & 0,392 & 1,95 & 0,051 \\
\hline \multicolumn{2}{|c|}{ Kreditgewerbe } & 0,757 & 2,48 & 0,013 \\
\hline \multicolumn{2}{|c|}{ Versicherungen } & 0,465 & 1,30 & 0,194 \\
\hline \multicolumn{2}{|c|}{ Gaststätten und Heime } & 0,651 & 3,59 & 0,000 \\
\hline Bildung und & lage & 0,661 & 3,29 & 0,001 \\
\hline Gesundheits & sen & 0,919 & 4,63 & 0,000 \\
\hline Freie Berufe & & 0,387 & 2,13 & 0,033 \\
\hline Sonstige Die & leistungen & 0,299 & 1,20 & 0,230 \\
\hline Organisation & ohne Erwerbscharakter/Staat & 0,498 & 2,93 & 0,003 \\
\hline Anteil qualifizi & e Beschäftigte (in \%) & 0,947 & 8,61 & 0,000 \\
\hline Anteil weiblich & Beschäftigte (in \%) & $-0,058$ & $-0,47$ & 0,641 \\
\hline Anteil Auszub & ende (in \%) & 0,248 & 0,69 & 0,488 \\
\hline Betriebsgröße & ummy) ${ }^{\star}$ & Referenzka & $100 \mathrm{bi}$ & chäftigte \\
\hline 1 bis $4 \mathrm{Bescl}$ & igte & $-1,571$ & $-12,05$ & 0,000 \\
\hline 5 bis $9 \mathrm{Besch}$ & igte & $-1,239$ & $-10,32$ & 0,000 \\
\hline 10 bis $19 \mathrm{Be}$ & äftigte & $-0,985$ & $-8,12$ & 0,000 \\
\hline 20 bis $49 \mathrm{Be}$ & äftigte & $-0,820$ & $-7,48$ & 0,000 \\
\hline 50 bis $99 \mathrm{Be}$ & äftigte & $-0,368$ & $-3,02$ & 0,003 \\
\hline 200 bis 499 & chäftigte & 0,083 & 0,65 & 0,518 \\
\hline 500 bis 999 & chäftigte & 0,207 & 1,30 & 0,194 \\
\hline 1000 bis 499 & eschäftigte & 0,678 & 3,25 & 0,001 \\
\hline Technische Al & gen auf neuem Stand (Dummy) & 0,126 & 2,03 & 0,042 \\
\hline Investitionen i & Kommunikationstechnik (Dummy) & 0,391 & 6,57 & 0,000 \\
\hline Überstunden ( & mmy) & 0,168 & 2,79 & 0,005 \\
\hline Fachkräftema & (Dummy) & 0,115 & 1,48 & 0,140 \\
\hline Konstante & & 0,071 & 0,27 & 0,787 \\
\hline${ }^{*}$ Betriebsgröße & 00 u. m. Beschäftigte aufgrund von & nearität ausg & sen & \\
\hline $\begin{array}{l}\mathrm{N} \\
\text { LR chi2(39) } \\
\text { Prob > chi2 } \\
\text { Log likelihood } \\
\text { Pseudo R2 }\end{array}$ & $\begin{array}{ll}= & 2803 \\
= & 1096,37 \\
= & 0,0000 \\
= & -1308,1443 \\
= & 0,2953 \\
\end{array}$ & & & \\
\hline
\end{tabular}

Quelle: LIAB-Längsschnitt, Wellen 1997 bis 2004, eigene Berechnungen 
Tab. 16: Probit-Schätzung der Finanzierung von internen Kursen

\begin{tabular}{|c|c|c|c|c|}
\hline \multicolumn{2}{|c|}{ Weiterbildung: interne Kurse } & Koeffizient & $\mathbf{Z}$ & $P>|z|$ \\
\hline \multicolumn{2}{|c|}{ Altersstruktur 1997 (in \%) } & \multicolumn{3}{|c|}{ Referenzkategorie: Anteil bis 25-Jährige } \\
\hline \multicolumn{2}{|c|}{ Anteil 26- bis 30-Jährige } & $-0,282$ & $-0,90$ & 0,368 \\
\hline \multicolumn{2}{|c|}{ Anteil 31- bis 35-Jährige } & 0,156 & 0,55 & 0,583 \\
\hline \multicolumn{2}{|c|}{ Anteil 36- bis 40-Jährige } & $-1,061$ & $-3,17$ & 0,002 \\
\hline \multicolumn{2}{|c|}{ Anteil 41- bis 45-Jährige } & $-0,447$ & $-1,35$ & 0,177 \\
\hline \multicolumn{2}{|c|}{ Anteil 46- bis 50 -Jährige } & $-0,761$ & $-2,28$ & 0,022 \\
\hline \multicolumn{2}{|c|}{ Anteil 51- bis 55-Jährige } & $-0,585$ & $-1,46$ & 0,144 \\
\hline \multicolumn{2}{|c|}{ Anteil 56- bis 60 -Jährige } & $-0,984$ & $-2,30$ & 0,021 \\
\hline \multicolumn{2}{|c|}{ Anteil 61- bis 65-Jährige } & $-0,661$ & $-0,60$ & 0,549 \\
\hline \multicolumn{2}{|c|}{ Anteil über 65-Jährige } & $-3,814$ & $-1,28$ & 0,199 \\
\hline \multicolumn{2}{|c|}{ Branche } & \multicolumn{3}{|c|}{ Referenzkategorie: Land- und Forstwirtschaft } \\
\hline \multicolumn{2}{|c|}{ Energie und Bergbau } & 0,668 & 2,44 & 0,015 \\
\hline \multicolumn{2}{|c|}{ Grundstoffverarbeitung } & 0,436 & 2,10 & 0,035 \\
\hline \multicolumn{2}{|c|}{ Investitionsgüter } & 0,451 & 2,34 & 0,019 \\
\hline \multicolumn{2}{|c|}{ Verbrauchsgüter } & 0,203 & 0,99 & 0,324 \\
\hline \multicolumn{2}{|c|}{ Baugewerbe } & $-0,012$ & $-0,06$ & 0,953 \\
\hline \multicolumn{2}{|c|}{ Handel } & 0,524 & 2,75 & 0,006 \\
\hline \multicolumn{2}{|c|}{ Verkehr und Nachrichten } & 0,321 & 1,41 & 0,158 \\
\hline \multicolumn{2}{|c|}{ Kreditgewerbe } & 1,223 & 3,83 & 0,000 \\
\hline \multicolumn{2}{|c|}{ Versicherungen } & 0,862 & 2,27 & 0,023 \\
\hline \multicolumn{2}{|c|}{ Gaststätten und Heime } & 0,691 & 3,36 & 0,001 \\
\hline Bildung und & lage & 0,446 & 2,12 & 0,034 \\
\hline Gesundheits & sen & 0,244 & 1,12 & 0,264 \\
\hline Freie Berufe & & 0,248 & 1,21 & 0,228 \\
\hline Sonstige Die & leistungen & 0,946 & 3,43 & 0,001 \\
\hline Organisation & ohne Erwerbscharakter/Staat & 0,221 & 1,15 & 0,248 \\
\hline Anteil qualifizi & e Beschäftigte (in \%) & 0,814 & 7,31 & 0,000 \\
\hline Anteil weiblich & Beschäftigte (in \%) & 0,242 & 1,85 & 0,064 \\
\hline Anteil Auszub & ende (in \%) & 0,047 & 0,13 & 0,896 \\
\hline Betriebsgröße & ummy) ${ }^{\star}$ & Referenzka & $100 \mathrm{bi}$ & chäftigte \\
\hline 1 bis $4 \mathrm{Bescl}$ & igte & $-1,771$ & $-12,17$ & 0,000 \\
\hline 5 bis $9 \mathrm{Besch}$ & igte & $-1,444$ & $-11,64$ & 0,000 \\
\hline 10 bis $19 \mathrm{Be}$ & äftigte & $-1,356$ & $-10,71$ & 0,000 \\
\hline 20 bis $49 \mathrm{Be}$ & äftigte & $-0,704$ & $-6,96$ & 0,000 \\
\hline 50 bis $99 \mathrm{Be}$ & äftigte & $-0,516$ & $-4,68$ & 0,000 \\
\hline 200 bis 499 & chäftigte & 0,118 & 1,11 & 0,266 \\
\hline 500 bis 999 & chäftigte & 0,698 & 5,05 & 0,000 \\
\hline 1000 bis 499 & eschäftigte & 1,508 & 7,30 & 0,000 \\
\hline Technische Al & gen auf neuem Stand (Dummy) & 0,294 & 4,60 & 0,000 \\
\hline Investitionen i & Kommunikationstechnik (Dummy) & 0,213 & 3,48 & 0,001 \\
\hline Überstunden ( & mmy) & 0,223 & 3,62 & 0,000 \\
\hline Fachkräftema & (Dummy)I & $-0,084$ & $-1,10$ & 0,273 \\
\hline Konstante & & $-0,396$ & $-1,35$ & 0,177 \\
\hline${ }^{*}$ Betriebsgröße & 00 u. m. Beschäftigte aufgrund von & nearität ausg & sen & \\
\hline $\begin{array}{l}\mathrm{N} \\
\text { LR chi2(39) } \\
\text { Prob > chi2 } \\
\text { Log likelihood } \\
\text { Pseudo R2 }\end{array}$ & $\begin{array}{ll}= & 2803 \\
= & 1344,26 \\
= & 0,0000 \\
= & -1255,2088 \\
= & 0,3487\end{array}$ & & & \\
\hline
\end{tabular}

Quelle: LIAB-Längsschnitt, Wellen 1997 bis 2004, eigene Berechnungen 
Tab. 17: $\quad$ Probit-Schätzung der Finanzierung von Weiterbildung am Arbeitsplatz

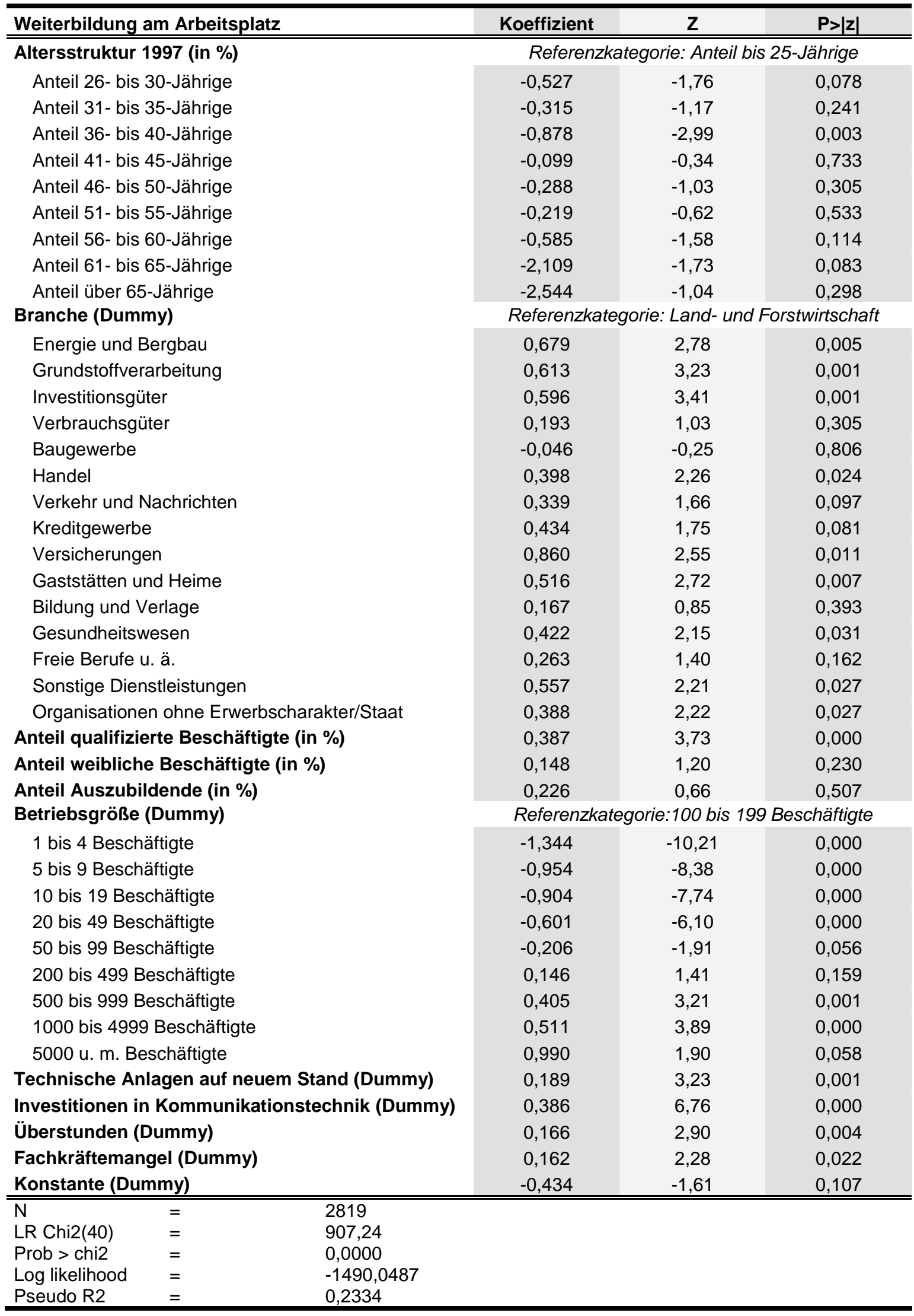

Quelle: LIAB-Längsschnitt, Wellen 1997 bis 2004, eigene Berechnungen 
Tab. 18: Matching-Ergebnisse: Finanzierung betrieblicher Weiterbildung 1997 - externe Kurse

\begin{tabular}{|c|c|c|c|c|c|c|c|}
\hline \multicolumn{2}{|c|}{ Anteil Mind.-50-Jähriger } & \multirow{2}{*}{$\begin{array}{c}\begin{array}{c}\text { Treatment- } \\
\text { gruppe }\end{array} \\
27,51 \%\end{array}$} & \multirow{2}{*}{$\begin{array}{c}\begin{array}{c}\text { Kontroll- } \\
\text { gruppe }\end{array} \\
27,89 \%\end{array}$} & \multirow{2}{*}{$\begin{array}{c}\text { Differenz } \\
-0,38 \%\end{array}$} & \multirow{2}{*}{$\begin{array}{c}\text { Std.abw. } \\
0,77 \%\end{array}$} & \multirow{2}{*}{$\frac{\text { t-Statistik }}{-0,49}$} & \multirow[t]{2}{*}{$P>|z|$} \\
\hline Methode & $\begin{array}{c}\text { Ohne } \\
\text { Matching }\end{array}$ & & & & & & \\
\hline $\begin{array}{l}\text { NN } \\
\text { LLR }\end{array}$ & $\begin{array}{l}\text { ATT } \\
\text { ATT }\end{array}$ & $\begin{array}{l}27,78 \% \\
27,78 \%\end{array}$ & $\begin{array}{l}29,97 \% \\
28,83 \%\end{array}$ & $\begin{array}{l}-2,20 \% \\
-1,05 \%\end{array}$ & $\begin{array}{l}2,32 \% \\
0,83 \%\end{array}$ & $\begin{array}{l}-0,95 \\
-1,27\end{array}$ & $\begin{array}{l}0,342 \\
0,205\end{array}$ \\
\hline
\end{tabular}

Quelle: LIAB Querschnitt, Wellen 1997 bis 2004, eigene Berechnungen

Tab. 19: $\quad$ Matching-Ergebnisse: Finanzierung betrieblicher Weiterbildung 1997 - interne Kurse

\begin{tabular}{|c|c|c|c|c|c|c|c|}
\hline \multicolumn{2}{|c|}{ Anteil Mind.-50-Jähriger } & \multirow{2}{*}{$\begin{array}{c}\begin{array}{c}\text { Treatment- } \\
\text { gruppe }\end{array} \\
26,68 \%\end{array}$} & \multirow{2}{*}{$\begin{array}{c}\begin{array}{c}\text { Kontroll- } \\
\text { gruppe }\end{array} \\
28,44 \%\end{array}$} & \multirow{2}{*}{$\begin{array}{c}\text { Differenz } \\
-1,76 \%\end{array}$} & \multirow{2}{*}{$\begin{array}{c}\text { Std.abw. } \\
0,75 \%\end{array}$} & \multirow{2}{*}{$\begin{array}{c}\text { t-Statistik } \\
-2,36\end{array}$} & \multirow[t]{2}{*}{$P>|z|$} \\
\hline Methode & $\begin{array}{c}\text { Ohne } \\
\text { Matching }\end{array}$ & & & & & & \\
\hline $\begin{array}{c}\mathrm{NN} \\
\operatorname{LLR}(0,8)\end{array}$ & $\begin{array}{l}\text { ATT } \\
\text { ATT }\end{array}$ & $\begin{array}{l}26,84 \% \\
26,84 \%\end{array}$ & $\begin{array}{l}27,31 \% \\
27,21 \%\end{array}$ & $\begin{array}{l}-0,48 \% \\
-0,36 \%\end{array}$ & $\begin{array}{l}1,90 \% \\
0,88 \%\end{array}$ & $\begin{array}{l}-0,25 \\
-0,41\end{array}$ & $\begin{array}{l}0,803 \\
0,681\end{array}$ \\
\hline
\end{tabular}

Quelle: LIAB Querschnitt, Wellen 1997 bis 2004, eigene Berechnungen

Tab. 20: $\quad$ Matching-Ergebnisse: Finanzierung betrieblicher Weiterbildung 1997 - Weiterbildung am Arbeitsplatz

\begin{tabular}{|c|c|c|c|c|c|c|c|}
\hline \multicolumn{2}{|c|}{ Anteil Mind.-50-Jähriger } & $\begin{array}{l}\text { Treatment- } \\
\text { gruppe }\end{array}$ & $\begin{array}{l}\text { Kontroll- } \\
\text { gruppe }\end{array}$ & Differenz & Std.abw & t-Statistik & $P>|z|$ \\
\hline Methode & $\begin{array}{c}\text { Ohne } \\
\text { Matching }\end{array}$ & $27,40 \%$ & $27,84 \%$ & $-0,44 \%$ & $0,74 \%$ & $-0,59$ & \\
\hline $\begin{array}{c}\mathrm{NN} \\
\operatorname{LLR}(0,8)\end{array}$ & $\begin{array}{l}\text { ATT } \\
\text { ATT }\end{array}$ & $\begin{array}{l}27,47 \% \\
27,47 \%\end{array}$ & $\begin{array}{l}28,12 \% \\
28,35 \%\end{array}$ & $\begin{array}{l}-0,66 \% \\
-0,88 \%\end{array}$ & $\begin{array}{l}1,09 \% \\
0,56 \%\end{array}$ & $\begin{array}{l}-0,60 \\
-1,56\end{array}$ & $\begin{array}{l}0,549 \\
0,118\end{array}$ \\
\hline
\end{tabular}

Quelle: LIAB Querschnitt, Wellen 1997 bis 2004, eigene Berechnungen 
Tab. 21: Verringerung der Verzerrung nach PS-Matching: Schätzungen durchgängiger Weiterbildung 1993 - 1995, nearest neighbor-Matching

\begin{tabular}{|c|c|c|c|c|c|c|}
\hline $\begin{array}{l}\text { Unmatched } \\
\text { Matched }\end{array}$ & $\begin{array}{c}\text { Mean } \\
\text { Treated }\end{array}$ & $\begin{array}{l}\text { Mean } \\
\text { Control }\end{array}$ & $\%$ Bias & $\begin{array}{c}\text { \%Bias- } \\
\text { Reduction }\end{array}$ & $\mathbf{t}$ & $\operatorname{Prob}(t>|t|)$ \\
\hline \multicolumn{7}{|l|}{ Altersstruktur 1993 (in \%) } \\
\hline Anteil 26- bis 30-Jährige & $\begin{array}{l}0,150 \\
0,148\end{array}$ & $\begin{array}{l}0,165 \\
0,140\end{array}$ & $\begin{array}{l}-11,3 \\
5,5\end{array}$ & 51,2 & $\begin{array}{r}-1,60 \\
1,25\end{array}$ & $\begin{array}{l}0,111 \\
0,212\end{array}$ \\
\hline Anteil 31- bis 35-Jährige & $\begin{array}{l}0,141 \\
0,141\end{array}$ & $\begin{array}{l}0,135 \\
0,132\end{array}$ & $\begin{array}{l}5,7 \\
7,3\end{array}$ & $-28,2$ & $\begin{array}{l}0,80 \\
1,80\end{array}$ & $\begin{array}{l}0,423 \\
0,072\end{array}$ \\
\hline Anteil 36- bis 40-Jährige & $\begin{array}{l}0,126 \\
0,126\end{array}$ & $\begin{array}{l}0,131 \\
0,122\end{array}$ & $\begin{array}{l}-4,1 \\
3,4\end{array}$ & 16,9 & $\begin{array}{l}-0,58 \\
1,16\end{array}$ & $\begin{array}{l}0,562 \\
0,248\end{array}$ \\
\hline Anteil 41- bis 45-Jährige & $\begin{array}{l}0,123 \\
0,124\end{array}$ & $\begin{array}{l}0,113 \\
0,123\end{array}$ & $\begin{array}{l}9,3 \\
0,4\end{array}$ & 95,8 & $\begin{array}{l}1,31 \\
0,09\end{array}$ & $\begin{array}{l}0,192 \\
0,925\end{array}$ \\
\hline Anteil 46- bis 50-Jährige & $\begin{array}{l}0,102 \\
0,102\end{array}$ & $\begin{array}{l}0,087 \\
0,108\end{array}$ & $\begin{array}{l}17,6 \\
-7,5\end{array}$ & 57,4 & $\begin{array}{r}2,48 \\
-1,80\end{array}$ & $\begin{array}{l}0,013 \\
0,072\end{array}$ \\
\hline Anteil 51- bis 55-Jährige & $\begin{array}{l}0,115 \\
0,117\end{array}$ & $\begin{array}{l}0,105 \\
0,127\end{array}$ & $\begin{array}{r}10,8 \\
-11,2\end{array}$ & $-3,5$ & $\begin{array}{c}1,52 \\
-2,39\end{array}$ & $\begin{array}{l}0,128 \\
0,017\end{array}$ \\
\hline Anteil 56- bis 60-Jährige & $\begin{array}{l}0,075 \\
0,076\end{array}$ & $\begin{array}{l}0,064 \\
0,078\end{array}$ & $\begin{array}{l}16,0 \\
-3,2\end{array}$ & 80,0 & $\begin{array}{c}2,24 \\
-0,60\end{array}$ & $\begin{array}{l}0,025 \\
0,550\end{array}$ \\
\hline Anteil 61- bis 65-Jährige & 0,014 & 0,011 & $\begin{array}{c}15,6 \\
49\end{array}$ & 68,4 & $\begin{array}{l}2,18 \\
0,77\end{array}$ & 0,030 \\
\hline Anteil über 65-Jährige & $\begin{array}{l}0,002 \\
0,002\end{array}$ & $\begin{array}{l}0,005 \\
0,001\end{array}$ & $\begin{array}{c}-18,0 \\
4,4\end{array}$ & 75,6 & $\begin{array}{c}-2,54 \\
1,66\end{array}$ & $\begin{array}{l}0,011 \\
0,097\end{array}$ \\
\hline \multicolumn{7}{|l|}{ Branche (Dummy) } \\
\hline Energie und Bergbau & $\begin{array}{l}0,033 \\
0,035\end{array}$ & $\begin{array}{l}0,005 \\
0,036\end{array}$ & $\begin{array}{l}20,2 \\
-1,0\end{array}$ & 95,1 & $\begin{array}{r}2,79 \\
-0,10\end{array}$ & $\begin{array}{l}0,005 \\
0,921\end{array}$ \\
\hline Grundstoffverarbeitung & $\begin{array}{l}0,118 \\
0,124\end{array}$ & $\begin{array}{l}0,071 \\
0,086\end{array}$ & $\begin{array}{l}16,2 \\
12,9\end{array}$ & 19,9 & $\begin{array}{l}2,25 \\
1,68\end{array}$ & $\begin{array}{l}0,025 \\
0,094\end{array}$ \\
\hline Investitionsgüter & $\begin{array}{l}0,211 \\
0,218\end{array}$ & $\begin{array}{l}0,105 \\
0,245\end{array}$ & $\begin{array}{l}29,3 \\
-7.5\end{array}$ & 74,5 & $\begin{array}{c}4,08 \\
-0,87\end{array}$ & $\begin{array}{l}0,000 \\
0,385\end{array}$ \\
\hline Verbrauchsgüter & $\begin{array}{l}0,043 \\
0.046\end{array}$ & $\begin{array}{l}0,126 \\
0.055\end{array}$ & $\begin{array}{l}-30,2 \\
-3,4\end{array}$ & 88,6 & $\begin{array}{l}-4,24 \\
-0,59\end{array}$ & $\begin{array}{l}0,000 \\
0,558\end{array}$ \\
\hline Baugewerbe & $\begin{array}{l}0,023 \\
0.024\end{array}$ & $\begin{array}{l}0,094 \\
0,026\end{array}$ & $\begin{array}{l}-30,9 \\
-0,6\end{array}$ & 98,1 & $\begin{array}{l}-4,34 \\
-0,12\end{array}$ & $\begin{array}{l}0,000 \\
0,906\end{array}$ \\
\hline Handel & $\begin{array}{l}0,068 \\
0,073\end{array}$ & $\begin{array}{l}0,149 \\
0,078\end{array}$ & $\begin{array}{l}-26,4 \\
-1,7\end{array}$ & 93,4 & $\begin{array}{l}-3,70 \\
-0,28\end{array}$ & $\begin{array}{l}0,000 \\
0,781\end{array}$ \\
\hline Verkehr und Nachrichten & $\begin{array}{l}0,028 \\
0,030\end{array}$ & $\begin{array}{l}0,037 \\
0,012\end{array}$ & $\begin{array}{l}-5,1 \\
9,9\end{array}$ & $-92,9$ & $\begin{array}{l}-0,72 \\
1,67\end{array}$ & $\begin{array}{l}0,472 \\
0,095\end{array}$ \\
\hline Kreditgewerbe & $\begin{array}{l}0,075 \\
0,040\end{array}$ & $\begin{array}{l}0,010 \\
0,051\end{array}$ & $\begin{array}{l}32,3 \\
-5,4\end{array}$ & 83,3 & $\begin{array}{c}4,48 \\
-0,70\end{array}$ & $\begin{array}{l}0,000 \\
0,483\end{array}$ \\
\hline Versicherungen & $\begin{array}{l}0,020 \\
0,022\end{array}$ & $\begin{array}{l}0,010 \\
0,009\end{array}$ & $\begin{array}{l}7,8 \\
9,9\end{array}$ & $-26,6$ & $\begin{array}{l}1,09 \\
1,34\end{array}$ & $\begin{array}{l}0,277 \\
0,182\end{array}$ \\
\hline Gaststätten und Heime & $\begin{array}{l}0,025 \\
0,027\end{array}$ & $\begin{array}{l}0,063 \\
0,027\end{array}$ & $\begin{array}{l}-18,5 \\
0,0\end{array}$ & 100,0 & $\begin{array}{l}-2,59 \\
0,00\end{array}$ & $\begin{array}{l}0,010 \\
1,000\end{array}$ \\
\hline Bildung und Verlage & $\begin{array}{l}0,045 \\
0,046\end{array}$ & $\begin{array}{l}0,031 \\
0,084\end{array}$ & $\begin{array}{c}7,1 \\
-19,7\end{array}$ & $-175,5$ & $\begin{array}{r}1,00 \\
-2,09\end{array}$ & $\begin{array}{l}0,320 \\
0,037\end{array}$ \\
\hline Gesundheitswesen & $\begin{array}{l}0,100 \\
0,089\end{array}$ & $\begin{array}{l}0,039 \\
0,038\end{array}$ & $\begin{array}{l}24,1 \\
20 ?\end{array}$ & 16,0 & $\begin{array}{l}3,35 \\
2,88\end{array}$ & 0,001 \\
\hline Freie Berufe u. ä. & 0,025 & 0,071 & $\begin{array}{l}2,2,5 \\
-13,3\end{array}$ & 38,0 & $\begin{array}{l}-3,01 \\
-194\end{array}$ & 0,003 \\
\hline Sonstige Dienstleistungen & $\begin{array}{l}0,003 \\
0,003\end{array}$ & $\begin{array}{l}0,013 \\
0,005\end{array}$ & $\begin{array}{c}-12,0 \\
-3,1\end{array}$ & 74,5 & $\begin{array}{l}-1,69 \\
-0,58\end{array}$ & $\begin{array}{l}0,091 \\
0,564\end{array}$ \\
\hline $\begin{array}{l}\text { Organisationen ohne Er- } \\
\text { werbscharakter/Staat }\end{array}$ & $\begin{array}{l}0,180 \\
0,191\end{array}$ & $\begin{array}{l}0,149 \\
0,187\end{array}$ & $\begin{array}{l}8,4 \\
1,1\end{array}$ & 87,1 & $\begin{array}{l}1,17 \\
0,14\end{array}$ & $\begin{array}{l}0,240 \\
0,888\end{array}$ \\
\hline
\end{tabular}




\begin{tabular}{|c|c|c|c|c|c|c|}
\hline $\begin{array}{l}\text { Unmatched } \\
\text { Matched }\end{array}$ & $\begin{array}{l}\text { Mean } \\
\text { Treated } \\
\end{array}$ & $\begin{array}{l}\text { Mean } \\
\text { Control } \\
\end{array}$ & \%Bias & $\begin{array}{c}\text { \%Bias- } \\
\text { Reduction } \\
\end{array}$ & $\mathbf{t}$ & $\operatorname{Prob}(t>|t|)$ \\
\hline $\begin{array}{l}\text { Anteil qualifizierte Beschäf- } \\
\text { tigte (in \%) }\end{array}$ & $\begin{array}{l}0,484 \\
0,460\end{array}$ & $\begin{array}{l}0,306 \\
0,486\end{array}$ & $\begin{array}{l}62,1 \\
-8,8\end{array}$ & 85,8 & $\begin{array}{r}8,68 \\
-1,17\end{array}$ & $\begin{array}{l}0,000 \\
0,241\end{array}$ \\
\hline $\begin{array}{l}\text { Anteil weibliche Beschäftigte } \\
\text { (in \%) }\end{array}$ & $\begin{array}{l}0,420 \\
0,408\end{array}$ & $\begin{array}{l}0,408 \\
0,383\end{array}$ & $\begin{array}{l}4,5 \\
8,9\end{array}$ & $-98,9$ & $\begin{array}{l}0,63 \\
1,32\end{array}$ & $\begin{array}{l}0,529 \\
0,187\end{array}$ \\
\hline Anteil Auszubildende (in \%) & $\begin{array}{l}0,049 \\
0,047\end{array}$ & $\begin{array}{l}0,047 \\
0,057\end{array}$ & $\begin{array}{c}1,7 \\
-13,6\end{array}$ & $-695,7$ & $\begin{array}{r}0,24 \\
-1,59\end{array}$ & $\begin{array}{l}0,810 \\
0,111\end{array}$ \\
\hline Betriebsgröße & & & & & & \\
\hline 1 bis 4 Beschäftigte & $\begin{array}{l}0,005 \\
0,005\end{array}$ & $\begin{array}{l}0,126 \\
0,005\end{array}$ & $\begin{array}{c}-50,3 \\
0,0\end{array}$ & 100,0 & $\begin{array}{c}-7,09 \\
0,00\end{array}$ & $\begin{array}{l}0,000 \\
1,000\end{array}$ \\
\hline 5 bis 9 Beschäftigte & $\begin{array}{l}0,028 \\
0,030\end{array}$ & $\begin{array}{l}0,199 \\
0,012\end{array}$ & $\begin{array}{c}-56,1 \\
5,7\end{array}$ & 89,8 & $\begin{array}{r}-7,90 \\
1,67\end{array}$ & $\begin{array}{l}0,000 \\
0,095\end{array}$ \\
\hline 10 bis 19 Beschäftigte & $\begin{array}{l}0,020 \\
0,022\end{array}$ & $\begin{array}{l}0,136 \\
0,022\end{array}$ & $\begin{array}{c}-44,3 \\
0,0\end{array}$ & 100,0 & $\begin{array}{l}-6,23 \\
0,00\end{array}$ & $\begin{array}{l}0,000 \\
1,000\end{array}$ \\
\hline 20 bis 49 Beschäftigte & $\begin{array}{l}0,088 \\
0,094\end{array}$ & $\begin{array}{l}0,181 \\
0,113\end{array}$ & $\begin{array}{l}-27,5 \\
-5,6\end{array}$ & 79,7 & $\begin{array}{l}-3,85 \\
-0,84\end{array}$ & $\begin{array}{l}0,000 \\
0,400\end{array}$ \\
\hline 50 bis 99 Beschäftigte & $\begin{array}{l}0,078 \\
0,084\end{array}$ & $\begin{array}{l}0,105 \\
0,101\end{array}$ & $\begin{array}{l}-9,4 \\
-6,1\end{array}$ & 35,2 & $\begin{array}{l}-1,31 \\
-0,82\end{array}$ & $\begin{array}{l}0,190 \\
0,410\end{array}$ \\
\hline 200 bis 499 Beschäftigte & $\begin{array}{l}0,183 \\
0,189\end{array}$ & $\begin{array}{l}0,092 \\
0,216\end{array}$ & $\begin{array}{l}26,7 \\
-7,9\end{array}$ & 70,5 & $\begin{array}{r}3,72 \\
-0,91\end{array}$ & $\begin{array}{l}0,000 \\
0,361\end{array}$ \\
\hline 500 bis 999 Beschäftigte & $\begin{array}{l}0,123 \\
0,113\end{array}$ & $\begin{array}{l}0,024 \\
0,108\end{array}$ & $\begin{array}{c}38,8 \\
2,1\end{array}$ & 94,6 & $\begin{array}{l}5,38 \\
0,23\end{array}$ & $\begin{array}{l}0,000 \\
0,815\end{array}$ \\
\hline 1000 bis 4999 Beschäftigte & $\begin{array}{l}0,331 \\
0,326\end{array}$ & $\begin{array}{l}0,058 \\
0,303\end{array}$ & $\begin{array}{c}73,5 \\
6,2\end{array}$ & 91,6 & $\begin{array}{c}10,20 \\
0,67\end{array}$ & $\begin{array}{l}0,000 \\
0,502\end{array}$ \\
\hline 5000 u. m. Beschäftigte & $\begin{array}{l}0,043 \\
0,027\end{array}$ & $\begin{array}{l}0,003 \\
0,023\end{array}$ & $\begin{array}{c}27,1 \\
2,7\end{array}$ & 89,9 & $\begin{array}{l}3,75 \\
0,35\end{array}$ & $\begin{array}{l}0,000 \\
0,724\end{array}$ \\
\hline $\begin{array}{l}\text { Technische Anlagen auf neu- } \\
\text { em Stand (Dummy) }\end{array}$ & $\begin{array}{l}0,754 \\
0,741\end{array}$ & $\begin{array}{l}0,678 \\
0,720\end{array}$ & $\begin{array}{c}17,0 \\
4,8\end{array}$ & 71,8 & $\begin{array}{l}2,37 \\
0,66\end{array}$ & $\begin{array}{l}0,018 \\
0,509\end{array}$ \\
\hline $\begin{array}{l}\text { Investitionen in Kommunika- } \\
\text { tionstechnik (Dummy) }\end{array}$ & $\begin{array}{l}0,797 \\
0,784\end{array}$ & $\begin{array}{l}0,466 \\
0,725\end{array}$ & $\begin{array}{l}73,0 \\
13,1\end{array}$ & 82,1 & $\begin{array}{c}10,22 \\
1,88\end{array}$ & $\begin{array}{l}0,000 \\
0,061\end{array}$ \\
\hline Überstunden (Dummy) & $\begin{array}{l}0,800 \\
0,787\end{array}$ & $\begin{array}{l}0,510 \\
0,819\end{array}$ & $\begin{array}{l}63,7 \\
-7,1\end{array}$ & 88,8 & $\begin{array}{r}8,93 \\
-1,11\end{array}$ & $\begin{array}{l}0,000 \\
0,268\end{array}$ \\
\hline Fachkräftemangel (Dummy) & $\begin{array}{l}0,388 \\
0,372\end{array}$ & $\begin{array}{l}0,304 \\
0,406\end{array}$ & $\begin{array}{l}17,9 \\
-7,1\end{array}$ & 60,3 & $\begin{array}{r}2,50 \\
-0,94\end{array}$ & $\begin{array}{l}0,013 \\
0,347\end{array}$ \\
\hline Betriebsrat (Dummy) & $\begin{array}{l}0,850 \\
0,841\end{array}$ & $\begin{array}{l}0,385 \\
0,858\end{array}$ & $\begin{array}{c}108,7 \\
-4,1\end{array}$ & 96,2 & $\begin{array}{l}15,24 \\
-0,67\end{array}$ & $\begin{array}{l}0,000 \\
0,505\end{array}$ \\
\hline
\end{tabular}

Quelle: LIAB-Längsschnitt, Wellen 1993 bis 2004, eigene Berechnungen 
Tab. 22: Verringerung der Verzerrung nach PS-Matching: Schätzungen durchgängiger Weiterbildung 1993 - 1995, LLR-Matching

\begin{tabular}{|c|c|c|c|c|c|c|}
\hline $\begin{array}{l}\text { Unmatched } \\
\text { Matched }\end{array}$ & $\begin{array}{c}\text { Mean } \\
\text { Treated } \\
\end{array}$ & $\begin{array}{l}\text { Mean } \\
\text { Control } \\
\end{array}$ & \%Bias & $\begin{array}{c}\text { \%Bias- } \\
\text { Reduction } \\
\end{array}$ & $\mathbf{t}$ & $\operatorname{Prob}(t>|t|)$ \\
\hline \multicolumn{7}{|l|}{ Altersstruktur 1993 (in \%) } \\
\hline \multirow{2}{*}{ Anteil 26- bis 30-Jährige } & 0,150 & 0,165 & $-11,3$ & \multirow{2}{*}{67,9} & $-1,60$ & 0,111 \\
\hline & 0,148 & 0,143 & 3,6 & & 0,82 & 0,413 \\
\hline \multirow{2}{*}{ Anteil 31- bis 35-Jährige } & 0,141 & 0,135 & 5,7 & \multirow{2}{*}{51,3} & 0,80 & 0,423 \\
\hline & 0,141 & 0,137 & 2,8 & & 0,69 & 0,490 \\
\hline \multirow{2}{*}{ Anteil 36- bis 40-Jährige } & 0,126 & 0,131 & $-4,1$ & \multirow{2}{*}{44,4} & $-0,58$ & 0,562 \\
\hline & 0,126 & 0,123 & 2,3 & & 0,74 & 0,461 \\
\hline \multirow{2}{*}{ Anteil 41- bis 45-Jährige } & 0,123 & 0,113 & 9,3 & \multirow{2}{*}{95,8} & 1,31 & 0,192 \\
\hline & 0,124 & 0,123 & 0,4 & & 0,10 & 0,917 \\
\hline \multirow{2}{*}{ Anteil 46- bis 50-Jährige } & 0,102 & 0,087 & 17,6 & \multirow{2}{*}{54,0} & 2,48 & 0,013 \\
\hline & 0,102 & 0,109 & $-8,1$ & & $-1,98$ & 0,048 \\
\hline \multirow{2}{*}{ Anteil 51- bis 55-Jährige } & 0,115 & 0,105 & 10,8 & \multirow{2}{*}{$-3,2$} & 1,52 & 0,128 \\
\hline & 0,117 & 0,127 & $-11,2$ & & $-2,37$ & 0,018 \\
\hline \multirow{2}{*}{ Anteil 56- bis 60-Jährige } & 0,075 & 0,064 & 16,0 & \multirow{2}{*}{98,0} & 2,24 & 0,025 \\
\hline & 0,076 & 0,076 & $-0,3$ & & $-0,06$ & 0,953 \\
\hline \multirow{2}{*}{ Anteil 61- bis 65-Jährige } & 0,014 & 0,011 & 15,6 & \multirow{2}{*}{39,2} & 2,18 & 0,030 \\
\hline & 0,015 & 0,013 & 9,5 & & 1,49 & 0,137 \\
\hline \multirow{2}{*}{ Anteil über 65-Jährige } & 0,002 & 0,005 & $-18,0$ & \multirow{2}{*}{63,8} & $-2,54$ & 0,011 \\
\hline & 0,002 & 0,001 & 6,5 & & 3,02 & 0,003 \\
\hline \multicolumn{7}{|l|}{ Branche (Dummy) } \\
\hline & 0,033 & 0,005 & 20,2 & & 2,79 & 0,005 \\
\hline Energie und Bergbau & 0,035 & 0,024 & 7,9 & 60,6 & 0,87 & 0,387 \\
\hline & 0,118 & 0,071 & 16,2 & & 2,25 & 0,025 \\
\hline Grundstoffverarbeitung & 0,124 & 0,100 & 8,3 & 48,5 & 1,05 & 0,295 \\
\hline & 0,211 & 0,105 & 29,3 & & 4,08 & 0,000 \\
\hline Investitionsgüter & 0,218 & 0,275 & $-15,7$ & 46,5 & $-1,79$ & 0,074 \\
\hline & 0,043 & 0,126 & $-30,2$ & & $-4,24$ & 0,000 \\
\hline Verbrauchsgüter & 0,046 & 0,040 & 2,0 & 93,5 & 0,36 & 0,718 \\
\hline & 0,023 & 0,094 & $-30,9$ & & $-4,34$ & 0,000 \\
\hline Baugewerbe & 0,024 & 0,024 & 0,0 & 100,0 & 0,00 & 1,000 \\
\hline & 0,068 & 0,149 & $-26,4$ & 934 & $-3,70$ & 0,000 \\
\hline Handel & 0,073 & 0,078 & $-1,7$ & 93,4 & $-0,28$ & 0,781 \\
\hline & 0,028 & 0,037 & $-5,1$ & & $-0,72$ & 0,472 \\
\hline Verkehr und Nachrichten & 0,030 & 0,011 & 10,7 & $-10 /, 8$ & 1,83 & 0,068 \\
\hline & 0,075 & 0,010 & 32,3 & & 4,48 & 0,000 \\
\hline Kreditgewerbe & 0,040 & 0,043 & $-1,3$ & 95,8 & $-0,18$ & 0,855 \\
\hline & 0,020 & 0,010 & 7,8 & & 1,09 & 0,277 \\
\hline Versicherungen & 0,022 & 0,005 & 13,2 & $-68,8$ & 1,91 & 0,056 \\
\hline & 0,025 & 0,063 & $-18,5$ & & $-2,59$ & 0,010 \\
\hline Gaststätten und Heime & 0,027 & 0,008 & 9,2 & 50,0 & 1,96 & 0,050 \\
\hline & 0,045 & 0,031 & 7,1 & & 1,00 & 0,320 \\
\hline Bildung und Verlage & 0,046 & 0,094 & $-25,3$ & $-254,2$ & $-2,60$ & 0,010 \\
\hline & 0,100 & 0,039 & 24,1 & & 3,35 & 0,001 \\
\hline Gesundheitswesen & 0,089 & 0,040 & 19,2 & 20,4 & 2,70 & 0,007 \\
\hline & 0,025 & 0,071 & $-21,5$ & & $-3,01$ & 0,003 \\
\hline Freie Berufe u. ä. & 0,027 & 0,038 & $-5,1$ & 76,4 & $-0,83$ & 0,407 \\
\hline & 0,003 & 0,013 & $-12,0$ & & $-1,69$ & 0,091 \\
\hline Sonstige Dienstleistungen & 0,003 & 0,005 & $-3,1$ & 74,5 & $-0,58$ & 0,564 \\
\hline Organisationen ohne Er- & 0,180 & 0,149 & 8,4 & & 1,17 & 0,240 \\
\hline werbscharakter/Staat & 0,191 & 0,205 & $-3,6$ & 56,9 & $-0,46$ & 0,646 \\
\hline
\end{tabular}




\begin{tabular}{|c|c|c|c|c|c|c|}
\hline $\begin{array}{l}\text { Unmatched } \\
\text { Matched }\end{array}$ & $\begin{array}{c}\text { Mean } \\
\text { Treated }\end{array}$ & $\begin{array}{l}\text { Mean } \\
\text { Control } \\
\end{array}$ & \%Bias & $\begin{array}{c}\text { \%Bias- } \\
\text { Reduction }\end{array}$ & $\mathbf{t}$ & $\operatorname{Prob}(t>|t|)$ \\
\hline $\begin{array}{l}\text { Anteil qualifizierte Beschäf- } \\
\text { tigte (in \%) }\end{array}$ & $\begin{array}{l}0,484 \\
0,460\end{array}$ & $\begin{array}{l}0,306 \\
0,466\end{array}$ & $\begin{array}{l}62,1 \\
-2,0\end{array}$ & 96,8 & $\begin{array}{l}8,68 \\
-0,27\end{array}$ & $\begin{array}{l}0,000 \\
0,788\end{array}$ \\
\hline $\begin{array}{l}\text { Anteil weibliche Beschäftigte } \\
\text { (in \%) }\end{array}$ & $\begin{array}{l}0,420 \\
0,408\end{array}$ & $\begin{array}{l}0,408 \\
0,365\end{array}$ & $\begin{array}{c}4,5 \\
15,4\end{array}$ & $-241,8$ & $\begin{array}{l}0,63 \\
2,26\end{array}$ & $\begin{array}{l}0,529 \\
0,024\end{array}$ \\
\hline Anteil Auszubildende (in \%) & $\begin{array}{l}0,049 \\
0,047\end{array}$ & $\begin{array}{l}0,047 \\
0,056\end{array}$ & $\begin{array}{c}1,7 \\
-11,1\end{array}$ & $-549,9$ & $\begin{array}{r}0,24 \\
-1,29\end{array}$ & $\begin{array}{l}0,810 \\
0,199\end{array}$ \\
\hline Betriebsgröße & & & & & & \\
\hline 1 bis 4 Beschäftigte & $\begin{array}{l}0,005 \\
0,005\end{array}$ & $\begin{array}{l}0,126 \\
0,003\end{array}$ & $\begin{array}{c}-50,3 \\
1,1\end{array}$ & 97,8 & $\begin{array}{c}-7,09 \\
0,58\end{array}$ & $\begin{array}{l}0,000 \\
0,564\end{array}$ \\
\hline 5 bis 9 Beschäftigte & $\begin{array}{l}0,028 \\
0,030\end{array}$ & $\begin{array}{l}0,199 \\
0,011\end{array}$ & $\begin{array}{c}-56,1 \\
6,2\end{array}$ & 89,0 & $\begin{array}{l}-7,90 \\
1,83\end{array}$ & $\begin{array}{l}0,000 \\
0,068\end{array}$ \\
\hline 10 bis 19 Beschäftigte & $\begin{array}{l}0,020 \\
0,022\end{array}$ & $\begin{array}{l}0,136 \\
0,030\end{array}$ & $\begin{array}{l}-44,3 \\
-3,1\end{array}$ & 93,0 & $\begin{array}{l}-6,23 \\
-0,70\end{array}$ & $\begin{array}{l}0,000 \\
0,486\end{array}$ \\
\hline 20 bis 49 Beschäftigte & $\begin{array}{l}0,088 \\
0,094\end{array}$ & $\begin{array}{l}0,181 \\
0,108\end{array}$ & $\begin{array}{l}-27,5 \\
-4,0\end{array}$ & 85,5 & $\begin{array}{l}-3,85 \\
-0,61\end{array}$ & $\begin{array}{l}0,000 \\
0,543\end{array}$ \\
\hline 50 bis 99 Beschäftigte & $\begin{array}{l}0,078 \\
0,084\end{array}$ & $\begin{array}{l}0,105 \\
0,086\end{array}$ & $\begin{array}{l}-9,4 \\
-0,9\end{array}$ & 90,0 & $\begin{array}{l}-1,31 \\
-0,13\end{array}$ & $\begin{array}{l}0,190 \\
0,895\end{array}$ \\
\hline 200 bis 499 Beschäftigte & $\begin{array}{l}0,183 \\
0,189\end{array}$ & $\begin{array}{l}0,092 \\
0,189\end{array}$ & $\begin{array}{c}26,7 \\
0,0\end{array}$ & 100,0 & $\begin{array}{l}3,72 \\
0,00\end{array}$ & $\begin{array}{l}0,000 \\
1,000\end{array}$ \\
\hline 500 bis 999 Beschäftigte & $\begin{array}{l}0,123 \\
0,113\end{array}$ & $\begin{array}{l}0,024 \\
0,102\end{array}$ & $\begin{array}{c}38,8 \\
4,2\end{array}$ & 89,1 & $\begin{array}{l}5,38 \\
0,47\end{array}$ & $\begin{array}{l}0,000 \\
0,636\end{array}$ \\
\hline 1000 bis 4999 Beschäftigte & $\begin{array}{l}0,331 \\
0,326\end{array}$ & $\begin{array}{l}0,058 \\
0,294\end{array}$ & $\begin{array}{c}73,5 \\
8,7\end{array}$ & 88,2 & $\begin{array}{c}10,20 \\
0,95\end{array}$ & $\begin{array}{l}0,000 \\
0,341\end{array}$ \\
\hline 5000 u. m. Beschäftigte & $\begin{array}{l}0,043 \\
0,027\end{array}$ & $\begin{array}{l}0,003 \\
0,038\end{array}$ & $\begin{array}{l}27,1 \\
-7,3\end{array}$ & 73,0 & $\begin{array}{r}3,75 \\
-0,83\end{array}$ & $\begin{array}{l}0,000 \\
0,407\end{array}$ \\
\hline $\begin{array}{l}\text { Technische Anlagen auf neu- } \\
\text { em Stand (Dummy) }\end{array}$ & $\begin{array}{l}0,754 \\
0,741\end{array}$ & $\begin{array}{l}0,678 \\
0,747\end{array}$ & $\begin{array}{l}17,0 \\
-1,2\end{array}$ & 92,9 & $\begin{array}{r}2,37 \\
-0,17\end{array}$ & $\begin{array}{l}0,018 \\
0,867\end{array}$ \\
\hline $\begin{array}{l}\text { Investitionen in Kommunika- } \\
\text { tionstechnik (Dummy) }\end{array}$ & $\begin{array}{l}0,797 \\
0,784\end{array}$ & $\begin{array}{l}0,466 \\
0,720\end{array}$ & $\begin{array}{l}73,0 \\
14,3\end{array}$ & 80,5 & $\begin{array}{c}10,22 \\
2,04\end{array}$ & $\begin{array}{l}0,000 \\
0,041\end{array}$ \\
\hline Überstunden (Dummy) & $\begin{array}{l}0,800 \\
0,787\end{array}$ & $\begin{array}{l}0,510 \\
0,841\end{array}$ & $\begin{array}{r}63,7 \\
-11,9\end{array}$ & 81,3 & $\begin{array}{r}8,93 \\
-1,89\end{array}$ & $\begin{array}{l}0,000 \\
0,059\end{array}$ \\
\hline Fachkräftemangel (Dummy) & $\begin{array}{l}0,388 \\
0,372\end{array}$ & $\begin{array}{l}0,304 \\
0,429\end{array}$ & $\begin{array}{r}17,9 \\
-11,9\end{array}$ & 33,3 & $\begin{array}{r}2,50 \\
-1,57\end{array}$ & $\begin{array}{l}0,013 \\
0,116\end{array}$ \\
\hline Betriebsrat (Dummy) & $\begin{array}{l}0,850 \\
0,841\end{array}$ & $\begin{array}{l}0,385 \\
0,863\end{array}$ & $\begin{array}{c}108,7 \\
-5,0\end{array}$ & 95,4 & $\begin{array}{l}15,24 \\
-0,83\end{array}$ & $\begin{array}{l}0,000 \\
0,409\end{array}$ \\
\hline
\end{tabular}

Quelle: LIAB-Längsschnitt, Wellen 1993 bis 2004, eigene Berechnungen 
Tab. 23: Verringerung der Verzerrung nach PS-Matching: Schätzungen Weiterbildung 1997, NN-Matching

\begin{tabular}{|c|c|c|c|c|c|c|}
\hline $\begin{array}{c}\text { Unmatched } \\
\text { Matched }\end{array}$ & $\begin{array}{c}\text { Mean } \\
\text { Treated } \\
\end{array}$ & $\begin{array}{l}\text { Mean } \\
\text { Control } \\
\end{array}$ & \%Bias & $\begin{array}{c}\text { \%Bias- } \\
\text { Reduction }\end{array}$ & $\mathbf{t}$ & $\operatorname{Prob}(t>|t|)$ \\
\hline \multicolumn{7}{|l|}{ Altersstruktur 1997 (in \%) } \\
\hline \multirow{2}{*}{ Anteil 26- bis 30-Jährige } & 0,113 & 0,126 & $-8,4$ & \multirow{2}{*}{85,8} & $-2,26$ & 0,024 \\
\hline & 0,113 & 0,111 & 1,2 & & 0,43 & 0,664 \\
\hline \multirow{2}{*}{ Anteil 31- bis 35-Jährige } & 0,151 & 0,152 & $-0,9$ & \multirow{2}{*}{75,9} & $-0,23$ & 0,815 \\
\hline & 0,151 & 0,151 & $-0,2$ & & $-0,08$ & 0,939 \\
\hline \multirow{2}{*}{ Anteil 36- bis 40-Jährige } & 0,144 & 0,155 & $-6,6$ & \multirow{2}{*}{78,0} & $-1,87$ & 0,062 \\
\hline & 0,143 & 0,146 & $-1,5$ & & $-0,64$ & 0,520 \\
\hline \multirow{2}{*}{ Anteil 41- bis 45-Jährige } & 0,141 & 0,127 & 9,7 & \multirow{2}{*}{51,9} & 2,66 & 0,008 \\
\hline & 0,141 & 0,134 & 4,7 & & 1,79 & 0,073 \\
\hline \multirow{2}{*}{ Anteil 46- bis 50-Jährige } & 0,120 & 0,124 & $-2,1$ & \multirow{2}{*}{$-46,8$} & $-0,59$ & 0,555 \\
\hline & 0,120 & 0,124 & $-3,0$ & & $-1,32$ & 0,188 \\
\hline \multirow{2}{*}{ Anteil 51- bis 55-Jährige } & 0,100 & 0,077 & 20,5 & \multirow{2}{*}{69,0} & 5,45 & 0,000 \\
\hline & 0,099 & 0,106 & $-6,3$ & & $-2,06$ & 0,040 \\
\hline \multirow{2}{*}{ Anteil 56- bis 60-Jährige } & 0,086 & 0,070 & 16,4 & \multirow[b]{2}{*}{87,9} & 4,18 & 0,000 \\
\hline & 0,086 & 0,088 & $-2,0$ & & $-0,64$ & 0,522 \\
\hline \multirow{2}{*}{ Anteil 61- bis 65-Jährige } & 0,011 & 0,010 & 1,6 & \multirow{2}{*}{$-14,7$} & 0,45 & 0,651 \\
\hline & 0,010 & 0,010 & 1,9 & & 0,81 & 0,417 \\
\hline \multirow{2}{*}{ Anteil über 65-Jährige } & 0,001 & 0,003 & $-8,0$ & \multirow{2}{*}{93,0} & $-2,29$ & 0,022 \\
\hline & 0,001 & 0,001 & 0,6 & & 0,35 & 0,730 \\
\hline \multicolumn{7}{|l|}{ Branche (Dummy) } \\
\hline Fneraie und Berabau & 0,027 & 0,001 & 22,1 & & 4,45 & 0,000 \\
\hline Energie und Bergbau & 0,013 & 0,016 & $-2,5$ & 88,5 & $-0,73$ & 0,463 \\
\hline & 0,063 & 0,049 & 5,8 & & 1,35 & 0,178 \\
\hline Grundstoftverarbeitung & 0,065 & 0,029 & 15,4 & $-166,5$ & 4,84 & 0,000 \\
\hline & 0,120 & 0,076 & 15,0 & & 3,42 & 0,001 \\
\hline Investitionsgüter & 0,113 & 0,139 & $-8,9$ & 40,4 & $-2,30$ & 0,021 \\
\hline & 0,048 & 0,094 & $-17,7$ & & $-4,53$ & 0,000 \\
\hline Verbrauchsgüter & 0,058 & 0,044 & 5,5 & 68,8 & 1,85 & 0,065 \\
\hline & 0,056 & 0,143 & $-29,3$ & & $-7,66$ & 0,000 \\
\hline Baugewerbe & 0,067 & 0,073 & $-1,7$ & 94,1 & $-0,58$ & 0,563 \\
\hline & 0,092 & 0,134 & $-13,3$ & & $-3,29$ & 0,001 \\
\hline Handel & 0,107 & 0,121 & $-4,6$ & 65,7 & $-1,31$ & 0,190 \\
\hline & 0,033 & 0,047 & $-6,9$ & 286 & $-1,70$ & 0,089 \\
\hline verkenr und Nachricnten & 0,037 & 0,027 & 4,9 & 28,6 & 1,58 & 0,114 \\
\hline Kreditaewerbe & 0,031 & 0,001 & 23,9 & 950 & 4,81 & 0,000 \\
\hline Kreaitgewerbe & 0,010 & 0,011 & $-1,2$ & 95,0 & $-0,43$ & 0,669 \\
\hline & 0,011 & 0,008 & 4,0 & -173 & 0,91 & 0,365 \\
\hline versicherungen & 0,008 & 0,003 & 4,6 & $-17,3$ & 1,75 & 0,080 \\
\hline & 0,057 & 0,091 & $-13,0$ & & $-3,24$ & 0,001 \\
\hline Gaststatten und Heıme & 0,069 & 0,079 & $-4,0$ & 68,9 & $-1,16$ & 0,246 \\
\hline & 0,071 & 0,029 & 19,3 & & 4,25 & 0,000 \\
\hline Bildung und Verlage & 0,075 & 0,122 & $-21,5$ & $-11,0$ & $-4,52$ & 0,000 \\
\hline & 0,079 & 0,052 & 10,8 & & 2,48 & 0,013 \\
\hline Gesundheitswesen & 0,061 & 0,064 & $-1,3$ & 87,6 & $-0,39$ & 0,693 \\
\hline & 0,061 & 0,087 & $-10,2$ & & $-2,52$ & 0,012 \\
\hline Freie Berufe u. ä. & 0,073 & 0,060 & 4,7 & 53,6 & 1,43 & 0,154 \\
\hline & 0,015 & 0,019 & $-2,7$ & & $-0,67$ & 0,505 \\
\hline Sonstige Dienstleistungen & 0,018 & 0,022 & $-3,0$ & $-10,0$ & $-0,81$ & 0,421 \\
\hline Organisationen ohne Er- & 0,213 & 0,099 & 31,9 & & 7,13 & 0,000 \\
\hline werbscharakter/Staat & 0,202 & 0,161 & 11,4 & 64,1 & 3,07 & 0,002 \\
\hline
\end{tabular}




\begin{tabular}{|c|c|c|c|c|c|c|}
\hline $\begin{array}{l}\text { Unmatched } \\
\text { Matched }\end{array}$ & $\begin{array}{l}\text { Mean } \\
\text { Treated }\end{array}$ & $\begin{array}{l}\text { Mean } \\
\text { Control } \\
\end{array}$ & \%Bias & $\begin{array}{c}\text { \%Bias- } \\
\text { Reduction }\end{array}$ & $\mathbf{t}$ & $\operatorname{Prob}(t>|t|)$ \\
\hline $\begin{array}{l}\text { Anteil qualifizierte Beschäf- } \\
\text { tigte (in \%) }\end{array}$ & $\begin{array}{l}0,451 \\
0,410\end{array}$ & $\begin{array}{l}0,226 \\
0,397\end{array}$ & $\begin{array}{c}73,5 \\
4,3\end{array}$ & 94,1 & $\begin{array}{c}17,02 \\
1,16\end{array}$ & $\begin{array}{l}0,000 \\
0,247\end{array}$ \\
\hline $\begin{array}{l}\text { Anteil weibliche Beschäftigte } \\
\text { (in \%) }\end{array}$ & $\begin{array}{l}0,472 \\
0,466\end{array}$ & $\begin{array}{l}0,422 \\
0,474\end{array}$ & $\begin{array}{l}16,4 \\
-2,6\end{array}$ & 84,0 & $\begin{array}{r}3,96 \\
-0,76\end{array}$ & $\begin{array}{l}0,000 \\
0,448\end{array}$ \\
\hline Anteil Auszubildende (in \%) & $\begin{array}{l}0,056 \\
0,057\end{array}$ & $\begin{array}{l}0,050 \\
0,055\end{array}$ & $\begin{array}{l}5,6 \\
1,0\end{array}$ & 81,5 & $\begin{array}{l}1,33 \\
0,26\end{array}$ & $\begin{array}{l}0,183 \\
0,794\end{array}$ \\
\hline Betriebsgröße & & & & & & \\
\hline 1 bis 4 Beschäftigte & $\begin{array}{l}0,050 \\
0,061\end{array}$ & $\begin{array}{l}0,249 \\
0,063\end{array}$ & $\begin{array}{l}-58,0 \\
-0,8\end{array}$ & 98,6 & $\begin{array}{l}-16,06 \\
-0,32\end{array}$ & $\begin{array}{l}0,000 \\
0,747\end{array}$ \\
\hline 5 bis 9 Beschäftigte & $\begin{array}{l}0,083 \\
0,100\end{array}$ & $\begin{array}{l}0,240 \\
0,100\end{array}$ & $\begin{array}{c}-43,9 \\
-0,1\end{array}$ & 99,8 & $\begin{array}{c}-11,55 \\
-0,03\end{array}$ & $\begin{array}{l}0,000 \\
0,977\end{array}$ \\
\hline 10 bis 19 Beschäftigte & $\begin{array}{l}0,079 \\
0,095\end{array}$ & $\begin{array}{l}0,166 \\
0,094\end{array}$ & $\begin{array}{c}-26,8 \\
0,5\end{array}$ & 98,3 & $\begin{array}{c}-6,88 \\
0,15\end{array}$ & $\begin{array}{l}0,000 \\
0,882\end{array}$ \\
\hline 20 bis 49 Beschäftigte & $\begin{array}{l}0,156 \\
0,189\end{array}$ & $\begin{array}{l}0,193 \\
0,208\end{array}$ & $\begin{array}{l}-9,8 \\
-5,1\end{array}$ & 48,5 & $\begin{array}{l}-2,39 \\
-1,39\end{array}$ & $\begin{array}{l}0,017 \\
0,164\end{array}$ \\
\hline 50 bis 99 Beschäftigte & $\begin{array}{l}0,115 \\
0,134\end{array}$ & $\begin{array}{l}0,073 \\
0,104\end{array}$ & $\begin{array}{l}14,2 \\
10,3\end{array}$ & 27,5 & $\begin{array}{l}3,26 \\
2,68\end{array}$ & $\begin{array}{l}0,001 \\
0,007\end{array}$ \\
\hline 200 bis 499 Beschäftigte & $\begin{array}{l}0,170 \\
0,168\end{array}$ & $\begin{array}{l}0,027 \\
0,181\end{array}$ & $\begin{array}{l}49,8 \\
-4,5\end{array}$ & 91,0 & $\begin{array}{l}10,39 \\
-0,98\end{array}$ & $\begin{array}{l}0,000 \\
0,326\end{array}$ \\
\hline 500 bis 999 Beschäftigte & $\begin{array}{l}0,101 \\
0,082\end{array}$ & $\begin{array}{l}0,011 \\
0,054\end{array}$ & $\begin{array}{l}39,6 \\
12,5\end{array}$ & 68,4 & $\begin{array}{l}8,16 \\
3,25\end{array}$ & $\begin{array}{l}0,000 \\
0,001\end{array}$ \\
\hline 1000 bis 4999 Beschäftigte & $\begin{array}{l}0,112 \\
0,017\end{array}$ & $\begin{array}{l}0,001 \\
0,034\end{array}$ & $\begin{array}{l}49,4 \\
-7,2\end{array}$ & 85,4 & $\begin{array}{l}9,86 \\
-2,97\end{array}$ & $\begin{array}{l}0,000 \\
0,003\end{array}$ \\
\hline 5000 u. m. Beschäftigte & $\begin{array}{l}0 \\
0\end{array}$ & $\begin{array}{l}0 \\
0\end{array}$ & $\begin{array}{l}0,0 \\
0,0\end{array}$ & - & $\begin{array}{l}0,00 \\
0,00\end{array}$ & \\
\hline $\begin{array}{l}\text { Technische Anlagen auf neu- } \\
\text { em Stand (Dummy) }\end{array}$ & $\begin{array}{l}0,685 \\
0,662\end{array}$ & $\begin{array}{l}0,626 \\
0,668\end{array}$ & $\begin{array}{l}12,6 \\
-1,1\end{array}$ & 90,9 & $\begin{array}{r}3,02 \\
-0,33\end{array}$ & $\begin{array}{l}0,003 \\
0,741\end{array}$ \\
\hline $\begin{array}{l}\text { Investitionen in Kommunika- } \\
\text { tionstechnik (Dummy) }\end{array}$ & $\begin{array}{l}0,635 \\
0,575\end{array}$ & $\begin{array}{l}0,322 \\
0,568\end{array}$ & $\begin{array}{c}65,9 \\
1,3\end{array}$ & 98,0 & $\begin{array}{c}15,61 \\
0,37\end{array}$ & $\begin{array}{l}0,000 \\
0,713\end{array}$ \\
\hline Überstunden (Dummy) & $\begin{array}{l}0,575 \\
0,530\end{array}$ & $\begin{array}{l}0,401 \\
0,559\end{array}$ & $\begin{array}{l}35,4 \\
-5,9\end{array}$ & 83,3 & $\begin{array}{r}8,42 \\
-1,69\end{array}$ & $\begin{array}{l}0,000 \\
0,091\end{array}$ \\
\hline Fachkräftemangel (Dummy) & $\begin{array}{l}0,185 \\
0,184\end{array}$ & $\begin{array}{l}0,150 \\
0,174\end{array}$ & $\begin{array}{l}9,4 \\
2,6\end{array}$ & 72,5 & $\begin{array}{l}2,19 \\
0,72\end{array}$ & $\begin{array}{l}0,029 \\
0,470\end{array}$ \\
\hline
\end{tabular}

Quelle: LIAB-Längsschnitt, Wellen 1997 bis 2004, eigene Berechnungen 
Tab. 24: Verringerung der Verzerrung nach PS-Matching: Schätzungen Weiterbildung 1997, LLR-Matching

\begin{tabular}{|c|c|c|c|c|c|c|}
\hline $\begin{array}{c}\text { Unmatched } \\
\text { Matched }\end{array}$ & $\begin{array}{c}\text { Mean } \\
\text { Treated } \\
\end{array}$ & $\begin{array}{l}\text { Mean } \\
\text { Control } \\
\end{array}$ & \%Bias & $\begin{array}{c}\text { \%Bias- } \\
\text { Reduction }\end{array}$ & $\mathbf{t}$ & $\operatorname{Prob}(t>|t|)$ \\
\hline \multicolumn{7}{|l|}{ Altersstruktur 1997 (in \%) } \\
\hline Anteil 26- bis 30-Jährige & $\begin{array}{l}0,113 \\
0,113\end{array}$ & $\begin{array}{l}0,126 \\
0,115\end{array}$ & $\begin{array}{l}-8,4 \\
-1,7\end{array}$ & 79,4 & $\begin{array}{l}-2,26 \\
-0,61\end{array}$ & $\begin{array}{l}0,024 \\
0,545\end{array}$ \\
\hline Anteil 31- bis 35-Jährige & $\begin{array}{l}0,151 \\
0,151\end{array}$ & $\begin{array}{l}0,152 \\
0,149\end{array}$ & $\begin{array}{c}-0,9 \\
0,9\end{array}$ & $-2,0$ & $\begin{array}{l}-0,23 \\
0,33\end{array}$ & $\begin{array}{l}0,815 \\
0,742\end{array}$ \\
\hline Anteil 36- bis 40-Jährige & $\begin{array}{l}0,144 \\
0,143\end{array}$ & $\begin{array}{l}0,155 \\
0,147\end{array}$ & $\begin{array}{l}-6,6 \\
-2,0\end{array}$ & 69,4 & $\begin{array}{l}-1,87 \\
-0.88\end{array}$ & $\begin{array}{l}0,062 \\
0,377\end{array}$ \\
\hline Anteil 41- bis 45-Jährige & $\begin{array}{l}0,141 \\
0,141\end{array}$ & $\begin{array}{l}0,127 \\
0,131\end{array}$ & $\begin{array}{l}9,7 \\
7,0\end{array}$ & 28,0 & $\begin{array}{l}2,66 \\
2,74\end{array}$ & $\begin{array}{l}0,008 \\
0,006\end{array}$ \\
\hline Anteil 46- bis 50-Jährige & $\begin{array}{l}0,120 \\
0,120\end{array}$ & $\begin{array}{l}0,124 \\
0,123\end{array}$ & $\begin{array}{l}-2,1 \\
-2,0\end{array}$ & 2,7 & $\begin{array}{r}-0,59 \\
-0,88\end{array}$ & $\begin{array}{l}0,555 \\
0,381\end{array}$ \\
\hline Anteil 51- bis 55-Jährige & $\begin{array}{l}0,100 \\
0,099\end{array}$ & $\begin{array}{l}0,077 \\
0,110\end{array}$ & $\begin{array}{r}20,5 \\
-10,1\end{array}$ & 50,6 & $\begin{array}{l}5,45 \\
-3,11\end{array}$ & $\begin{array}{l}0,000 \\
0,002\end{array}$ \\
\hline Anteil 56- bis 60-Jährige & $\begin{array}{l}0,086 \\
0,086\end{array}$ & $\begin{array}{l}0,070 \\
0,092\end{array}$ & $\begin{array}{l}16,4 \\
-5,5\end{array}$ & 66,8 & $\begin{array}{r}4,18 \\
-1,74\end{array}$ & $\begin{array}{l}0,000 \\
0,082\end{array}$ \\
\hline Anteil 61- bis 65-Jährige & $\begin{array}{l}0,011 \\
0,010\end{array}$ & $\begin{array}{l}0,010 \\
0,011\end{array}$ & $\begin{array}{r}1,6 \\
-1,6\end{array}$ & 3,2 & $\begin{array}{r}0,45 \\
-0,72\end{array}$ & $\begin{array}{l}0,651 \\
0,471\end{array}$ \\
\hline Anteil über 65-Jährige & $\begin{array}{l}0,001 \\
0,001\end{array}$ & $\begin{array}{l}0,003 \\
0,002\end{array}$ & $\begin{array}{l}-8,0 \\
-1,0\end{array}$ & 87,4 & $\begin{array}{l}-2,29 \\
-0,61\end{array}$ & $\begin{array}{l}0,022 \\
0,540\end{array}$ \\
\hline \multicolumn{7}{|l|}{ Branche (Dummy) } \\
\hline Energie und Bergbau & $\begin{array}{l}0,027 \\
0,013\end{array}$ & $\begin{array}{l}0,001 \\
0,013\end{array}$ & $\begin{array}{c}22,1 \\
0,0\end{array}$ & 100,0 & $\begin{array}{l}4,45 \\
0,00\end{array}$ & $\begin{array}{l}0,000 \\
1,000\end{array}$ \\
\hline Grundstoffverarbeitung & $\begin{array}{l}0,063 \\
0,065\end{array}$ & $\begin{array}{l}0,049 \\
0,028\end{array}$ & $\begin{array}{c}5,8 \\
16,2\end{array}$ & $-180,1$ & $\begin{array}{l}1,35 \\
5,13\end{array}$ & $\begin{array}{l}0,178 \\
0,000\end{array}$ \\
\hline Investitionsgüter & $\begin{array}{l}0,120 \\
0,113\end{array}$ & $\begin{array}{l}0,076 \\
0,138\end{array}$ & $\begin{array}{l}15,0 \\
-8,5\end{array}$ & 43,1 & $\begin{array}{r}3,42 \\
-2,20\end{array}$ & $\begin{array}{l}0,001 \\
0,028\end{array}$ \\
\hline Verbrauchsgüter & $\begin{array}{l}0,048 \\
0,058\end{array}$ & $\begin{array}{l}0,094 \\
0,035\end{array}$ & $\begin{array}{c}-17,7 \\
9,2\end{array}$ & 48,2 & $\begin{array}{c}-4,53 \\
3,21\end{array}$ & $\begin{array}{l}0,000 \\
0,001\end{array}$ \\
\hline Baugewerbe & $\begin{array}{l}0,056 \\
0,067\end{array}$ & $\begin{array}{l}0,143 \\
0,070\end{array}$ & $\begin{array}{c}-29,3 \\
-1,0\end{array}$ & 96,5 & $\begin{array}{l}-7,66 \\
-0,34\end{array}$ & $\begin{array}{l}0,000 \\
0,732\end{array}$ \\
\hline Handel & $\begin{array}{l}0,092 \\
0,107\end{array}$ & $\begin{array}{l}0,134 \\
0,109\end{array}$ & $\begin{array}{c}-13,3 \\
-0,8\end{array}$ & 94,3 & $\begin{array}{l}-3,29 \\
-0,22\end{array}$ & $\begin{array}{l}0,001 \\
0,823\end{array}$ \\
\hline Verkehr und Nachrichten & $\begin{array}{l}0,033 \\
0,037\end{array}$ & $\begin{array}{l}0,047 \\
0,026\end{array}$ & $\begin{array}{c}-6,9 \\
5,5\end{array}$ & 19,6 & $\begin{array}{c}-1,70 \\
1,79\end{array}$ & $\begin{array}{l}0,089 \\
0,073\end{array}$ \\
\hline Kreditgewerbe & $\begin{array}{l}0,031 \\
0,010\end{array}$ & $\begin{array}{l}0,001 \\
0,011\end{array}$ & $\begin{array}{l}23,9 \\
-1,4\end{array}$ & 94,0 & $\begin{array}{r}4,81 \\
-0,51\end{array}$ & $\begin{array}{l}0,000 \\
0,610\end{array}$ \\
\hline Versicherungen & $\begin{array}{l}0,011 \\
0,008\end{array}$ & $\begin{array}{l}0,008 \\
0,003\end{array}$ & $\begin{array}{l}4,0 \\
5,0\end{array}$ & $-25,2$ & $\begin{array}{l}0,91 \\
1,89\end{array}$ & $\begin{array}{l}0,365 \\
0,059\end{array}$ \\
\hline Gaststätten und Heime & $\begin{array}{l}0,057 \\
0,069\end{array}$ & $\begin{array}{l}0,091 \\
0,090\end{array}$ & $\begin{array}{c}-13,0 \\
-8,3\end{array}$ & 36,0 & $\begin{array}{l}-3,24 \\
-2,31\end{array}$ & $\begin{array}{l}0,001 \\
0,021\end{array}$ \\
\hline Bildung und Verlage & $\begin{array}{l}0,071 \\
0,075\end{array}$ & $\begin{array}{l}0,029 \\
0,160\end{array}$ & $\begin{array}{r}19,3 \\
-39,1\end{array}$ & $-102,0$ & $\begin{array}{r}4,25 \\
-7,66\end{array}$ & $\begin{array}{l}0,000 \\
0,000\end{array}$ \\
\hline Gesundheitswesen & $\begin{array}{l}0,079 \\
0,061\end{array}$ & $\begin{array}{l}0,052 \\
0,032\end{array}$ & $\begin{array}{l}10,8 \\
11,5\end{array}$ & $-5,9$ & $\begin{array}{l}2,48 \\
3,87\end{array}$ & $\begin{array}{l}0,013 \\
0,000\end{array}$ \\
\hline Freie Berufe u. ä. & $\begin{array}{l}0,061 \\
0,073\end{array}$ & $\begin{array}{l}0,087 \\
0,063\end{array}$ & $\begin{array}{c}-10,2 \\
3,9\end{array}$ & 61,5 & $\begin{array}{c}-2,52 \\
1,17\end{array}$ & $\begin{array}{l}0,012 \\
0,241\end{array}$ \\
\hline Sonstige Dienstleistungen & $\begin{array}{l}0,015 \\
0,018\end{array}$ & $\begin{array}{l}0,019 \\
0,023\end{array}$ & $\begin{array}{l}-2,7 \\
-4,2\end{array}$ & $-52,3$ & $\begin{array}{l}-0,67 \\
-1,09\end{array}$ & $\begin{array}{l}0,505 \\
0,274\end{array}$ \\
\hline $\begin{array}{l}\text { Organisationen ohne Er- } \\
\text { werbscharakter/Staat }\end{array}$ & $\begin{array}{l}0,213 \\
0,202\end{array}$ & $\begin{array}{l}0,099 \\
0,168\end{array}$ & $\begin{array}{c}31,9 \\
9,2\end{array}$ & 71,0 & $\begin{array}{l}7,13 \\
2,46\end{array}$ & $\begin{array}{l}0,000 \\
0,014\end{array}$ \\
\hline
\end{tabular}




\begin{tabular}{|c|c|c|c|c|c|c|}
\hline $\begin{array}{l}\text { Unmatched } \\
\text { Matched }\end{array}$ & $\begin{array}{l}\text { Mean } \\
\text { Treated }\end{array}$ & $\begin{array}{l}\text { Mean } \\
\text { Control } \\
\end{array}$ & \%Bias & $\begin{array}{c}\text { \%Bias- } \\
\text { Reduction }\end{array}$ & $\mathbf{t}$ & $\operatorname{Prob}(t>|t|)$ \\
\hline $\begin{array}{l}\text { Anteil qualifizierte Beschäf- } \\
\text { tigte (in \%) }\end{array}$ & $\begin{array}{l}0,451 \\
0,410\end{array}$ & $\begin{array}{l}0,226 \\
0,413\end{array}$ & $\begin{array}{l}73,5 \\
-0,9\end{array}$ & 98,7 & $\begin{array}{l}17,02 \\
-0,25\end{array}$ & $\begin{array}{l}0,000 \\
0,799\end{array}$ \\
\hline $\begin{array}{l}\text { Anteil weibliche Beschäftigte } \\
\text { (in \%) }\end{array}$ & $\begin{array}{l}0,472 \\
0,466\end{array}$ & $\begin{array}{l}0,422 \\
0,473\end{array}$ & $\begin{array}{l}16,4 \\
-2,3\end{array}$ & 85,7 & $\begin{array}{r}3,96 \\
-0,68\end{array}$ & $\begin{array}{l}0,000 \\
0,496\end{array}$ \\
\hline Anteil Auszubildende (in \%) & $\begin{array}{l}0,056 \\
0,057\end{array}$ & $\begin{array}{l}0,050 \\
0,054\end{array}$ & $\begin{array}{l}5,6 \\
2,1\end{array}$ & 61,7 & $\begin{array}{l}1,33 \\
0,54\end{array}$ & $\begin{array}{l}0,183 \\
0,591\end{array}$ \\
\hline Betriebsgröße & & & & & & \\
\hline 1 bis 4 Beschäftigte & $\begin{array}{l}0,050 \\
0,061\end{array}$ & $\begin{array}{l}0,249 \\
0,068\end{array}$ & $\begin{array}{l}-58,0 \\
-2,1\end{array}$ & 96,4 & $\begin{array}{l}-16,06 \\
-0,85\end{array}$ & $\begin{array}{l}0,000 \\
0,397\end{array}$ \\
\hline 5 bis 9 Beschäftigte & $\begin{array}{l}0,083 \\
0,100\end{array}$ & $\begin{array}{l}0,240 \\
0,096\end{array}$ & $\begin{array}{c}-43,9 \\
1,2\end{array}$ & 97,3 & $\begin{array}{c}-11,55 \\
0,41\end{array}$ & $\begin{array}{l}0,000 \\
0,683\end{array}$ \\
\hline 10 bis 19 Beschäftigte & $\begin{array}{l}0,079 \\
0,095\end{array}$ & $\begin{array}{l}0,166 \\
0,097\end{array}$ & $\begin{array}{l}-26,8 \\
-0,6\end{array}$ & 97,9 & $\begin{array}{l}-6,88 \\
-0,18\end{array}$ & $\begin{array}{l}0,000 \\
0,860\end{array}$ \\
\hline 20 bis 49 Beschäftigte & $\begin{array}{l}0,156 \\
0,189\end{array}$ & $\begin{array}{l}0,193 \\
0,196\end{array}$ & $\begin{array}{l}-9,8 \\
-1,7\end{array}$ & 82,3 & $\begin{array}{l}-2,39 \\
-0,48\end{array}$ & $\begin{array}{l}0,017 \\
0,628\end{array}$ \\
\hline 50 bis 99 Beschäftigte & $\begin{array}{l}0,115 \\
0,134\end{array}$ & $\begin{array}{l}0,073 \\
0,113\end{array}$ & $\begin{array}{c}14,2 \\
7,2\end{array}$ & 49,2 & $\begin{array}{l}3,26 \\
1,84\end{array}$ & $\begin{array}{l}0,001 \\
0,065\end{array}$ \\
\hline 200 bis 499 Beschäftigte & $\begin{array}{l}0,170 \\
0,168\end{array}$ & $\begin{array}{l}0,027 \\
0,217\end{array}$ & $\begin{array}{r}49,8 \\
-16,8\end{array}$ & 66,1 & $\begin{array}{l}10,39 \\
-3,57\end{array}$ & $\begin{array}{l}0,000 \\
0,000\end{array}$ \\
\hline 500 bis 999 Beschäftigte & $\begin{array}{l}0,101 \\
0,082\end{array}$ & $\begin{array}{l}0,011 \\
0,060\end{array}$ & $\begin{array}{c}39,6 \\
9,6\end{array}$ & 75,8 & $\begin{array}{l}8,16 \\
2,43\end{array}$ & $\begin{array}{l}0,000 \\
0,015\end{array}$ \\
\hline 1000 bis 4999 Beschäftigte & $\begin{array}{l}0,112 \\
0,017\end{array}$ & $\begin{array}{l}0,001 \\
0,006\end{array}$ & $\begin{array}{c}49,4 \\
5,1\end{array}$ & 89,7 & $\begin{array}{l}9,86 \\
3,06\end{array}$ & $\begin{array}{l}0,000 \\
0,002\end{array}$ \\
\hline 5000 u. m. Beschäftigte & $\begin{array}{l}0 \\
0\end{array}$ & $\begin{array}{l}0 \\
0\end{array}$ & $\begin{array}{l}0,0 \\
0,0\end{array}$ & 0,0 & $\begin{array}{l}0,00 \\
0,00\end{array}$ & \\
\hline $\begin{array}{l}\text { Technische Anlagen auf neu- } \\
\text { em Stand (Dummy) }\end{array}$ & $\begin{array}{l}0,685 \\
0,662\end{array}$ & $\begin{array}{l}0,626 \\
0,644\end{array}$ & $\begin{array}{c}12,6 \\
3,8\end{array}$ & 69,7 & $\begin{array}{l}3,02 \\
1,09\end{array}$ & $\begin{array}{l}0,003 \\
0,274\end{array}$ \\
\hline $\begin{array}{l}\text { Investitionen in Kommunika- } \\
\text { tionstechnik (Dummy) }\end{array}$ & $\begin{array}{l}0,635 \\
0,575\end{array}$ & $\begin{array}{l}0,322 \\
0,588\end{array}$ & $\begin{array}{l}65,9 \\
-2,8\end{array}$ & 95,8 & $\begin{array}{l}15,61 \\
-0,77\end{array}$ & $\begin{array}{l}0,000 \\
0,439\end{array}$ \\
\hline Überstunden (Dummy) & $\begin{array}{l}0,575 \\
0,530\end{array}$ & $\begin{array}{l}0,401 \\
0,584\end{array}$ & $\begin{array}{r}35,4 \\
-10,9\end{array}$ & 69,3 & $\begin{array}{r}8,42 \\
-3,11\end{array}$ & $\begin{array}{l}0,000 \\
0,002\end{array}$ \\
\hline Fachkräftemangel (Dummy) & $\begin{array}{l}0,185 \\
0,184\end{array}$ & $\begin{array}{l}0,150 \\
0,134\end{array}$ & $\begin{array}{c}9,4 \\
13,5\end{array}$ & $-44,6$ & $\begin{array}{l}2,19 \\
3,99\end{array}$ & $\begin{array}{l}0,029 \\
0,000\end{array}$ \\
\hline
\end{tabular}

Quelle: LIAB-Längsschnitt, Wellen 1997 bis 2004, eigene Berechnungen 


\section{Literatur}

Abowd, J. und F. Kramarz (1999), The analysis of labor markets using matched employeremployee data, in: O. Ashenfelter and D. Card (eds.): Handbook of Labor Economics, Vol. 3B, Elsevier, Amsterdam, S. 2567-2627.

Achtenhagen, C. (2004), Arbeitsmarktpolitische Maßnahmen zugunsten Älterer, Peter Lang Verlag, Frankfurt.

Addison, J.T., Schnabel, C. und J. Wagner (2001), Work councils in Germany: their effects on establishment performance, in: Oxford Economic papers, 53 (4), S. 659-694, Oxford.

Addison, J.T. und J. Teixeira (2003), The Economics of Employment Protection, in: Journal of Labor Research, 24, 85-128.

Ahituv, A. und J. Zeira (2000), Technical Progress and Early Retirement, Discussion paper 2614, CEPR.

Alda, H. (2005), Die Verknüpfungsqualität der LIAB-Daten, FDZ-Datenreport Nr. 1/2005.

Alda, H. und D. Herrlinger (2005a), LIAB-Datenhandbuch-Version 1.0, FDZ-Datenreport Nr. 7/2005, Nürnberg.

Alda, H. und D. Herrlinger (2005b), LIAB-Datenhandbuch -Version 1.0 - Anlageband I, FDZ-Datenreport Nr. 7/2005, Nürnberg.

Alda, H. und D. Herrlinger (2005c), LIAB-Datenhandbuch -Version 1.0 - Anlageband II, FDZ-Datenreport Nr. 7/2005, Nürnberg.

Allart, P., H. Alda und L. Bellmann (2005), Churning and Institutions, Churning and institutions - Dutch and German establishments compared with micro-level data, IAB Discussion Paper Nr. 12/2005, Nürnberg.

Anderson, K.H. und R.V. Burkhauser (1985), The Retirement-Health Nexus: A New Measure of an Old Puzzle, in: Journal of Human Resources, 21, S. 118-137.

Antolin, S. und S. Scarpetta (1998), Microeconomic analysis of the retirement decision: Germany, Working paper No. 204, Paris: OECD Economics Department.

Arnds, P. und Bonin, H. (2003), Frühverrentung in Deutschland: Ökonomische Anreize und institutionelle Strukturen, in: M. Herfurth, M. Kohli and K. F. Zimmermann (Hrsg.): Arbeit in einer alternden Gesellschaft, S. 65-91, Leske+Budrich, Leverkusen.

Autor, D. H., F. Levy, und R.J. Murnane (2001), The skill content of technological change: an empirical exploration, NBER Working Paper No. 8337, Cambridge (Mass.). 
Bailey, K. (1984), Asymptotic Equivalence Between the Cox Estimator and the General Maximum Likelihood Estimator of Regression and Survival Parameters, in: Annals of Statistics 12 (2), S. 730-736.

Bartel, A. und N. Sicherman (1993), Technological Change and retirement Decisions of Older Workers, in: Journal of Labour Economics, Bd. 11(1), S. 162-183.

Battistin, E. und E. Rettore (2003), Another Look at the Regression Discontinuity Design, cenmap Working Paper 01/03.

Bauer, T.; Bender S. und H. Bonin (2004), Dismissal Protection and Worker Flows in Small Establishments, IZA discussion paper No.1105, Bonn.

Bazzoli, G. (1985), The early retirement decision: New Empirical Evidence of the Influence of Health, in: Journal of Human Resources, Bd. 20 (2), S. 214-234.

Becker, G. S. (1964), Human Capital, NBER, Cambridge (Mass).

Becker, R. und K. Schömann (1999), Berufliche Weiterbildung und Einkommenschancen im Lebensverlauf: Empirische Befunde für Frauen und Männer in West- und Ostdeutschland, in D. Beer et al. (Hrsg.): Die wirtschaftlichen Folgen von Aus- und Weiterbildung. München.

Beckmann, M. (2002), Die Auswirkungen des betrieblichen Personalabbaus auf die Altersstruktur der Belegschaften in westdeutschen Unternehmen, in: Bellmann L. und A. Kölling (Hrsg.): Betrieblicher Wandel und Fachkräftebedarf, S. 133-171, Nürnberg.

Beckmann, M. und L. Bellmann (2002), Churning in deutschen Betrieben welche Rolle spielen technischer Fortschritt, organisatorische Änderungen und Personalstruktur?, in: L. Bellmann \& A. Kölling (Hrsg.), Betrieblicher Wandel und Fachkräftebedarf, Nürnberg: S. 133171 Reihe / Serie: Beiträge zur Arbeitsmarkt- und Berufsforschung Nr. 257.

Bellmann, L. und U. Leber (2004), Betriebliche Weiterbildung für ältere Arbeitnehmer, in: Arbeit und Beruf, Jg. 55, H. 1., S. 9-10.

Bellmann, L. und U. Leber (2005), Berufliche Weiterbildungsforschung - Datenlage, Forschungsfragen und ausgewählte Ergebnisse, in: Report. Literatur- und Forschungsreport Weiterbildung, Jg. 28 (2), S. 29-40.

Bender, S., D. Konietzka und P. Sopp (2000), Diskontiniutät im Erwerbsverlauf und betrieblicher Kontext, in: Kölner Zeitschrift für Soziologie und Sozialpsychologie, Bd. 52(3), S. 475499.

Bender, S., J. Preißler und C. Wübbeke (2000), Betriebliche Determinanten des Generationenaustausches in westdeutschen Betrieben - eine Untersuchung auf der Basis des IABEmployer-Employee-Datensatzes für die Jahre 1994 und 1995, in: R. George und O. Struck (Hrsg.): Generationenaustausch im Unternehmen, München u.a.: Hampp S. 209-225. 
Bergemann, A. und A. Mertens (2004), Job Stability Trends, Layoffs and Transitions to Unemployment: An Empirical Analysis for West Germany, IZA discussion paper No. 1368, Bonn.

Bergemann, A. und H. Schneider (1998), Ist der deutsche Arbeitsmarkt beweglicher geworden? - Eine Analyse anhand der Unternehmenszugehörigkeitsdauer in Westdeutschland, in. Wirtschaft im Wandel, Bd. 11.

Bertschek, I. (2004), Führt IT-Nutzung zu altersfeindlicher Reorganisation von Arbeit? Eine Untersuchung auf Grundlage der IKT-Umfrage des ZEW, in: B. Fitzenberger, W. Smolny und P. Winker (Hrsg.): Herausforderungen an den Wirtschaftsstandort Deutschland, Schriftenreihe des ZEW; Band 72, Mannheim.

Bispinck, R. und WSI-Tarifarchiv (2002), Tarifpolitik für ältere ArbeitnehmerInnen- eine Analyse von tariflichen Regelungen in ausgewählten Tarifbereichen, Elemente qualitativer Tarifpolitik Nr. 49, Düsseldorf.

Blau, D. (1994), Labor Force Dynamics of Older Men, in: Econometrica, Bd. 62, S. 117-156.

Blau, D. (1998), Labour Force Dynamics of Older Married Couples, in: Journal of Labour Economics, Bd. 17(3).

Blau, D. und D.B. Gilleskie (1998), A Dynamic Structural Model of Health Insurance and Retirement, manuscript. University of North Carolina at Chapel Hill.

Blau, D. und R. T. Riphahn (1998), Labour Force Transitions of Older Married Couples in Germany, in: Labour Economics 6(2), S. 229-251.

Blau, D. und T. Shvydko (2007), Labor Market Rigidities and the Employment Behavior of Older Workers, IZA working paper No. 2996, Bonn.

Bloemen, H. G. (2006): The Impact of Wealth on Job Exit Rates of Elderly Workers, IZA working paper No. 2247, Bonn.

Bloemen, H. G. (2008): Private Wealth and Job Exit at Older Age: A Random Effects Model, IZA working paper No. 3386, Bonn.

Blossfeld, H.-P., A. Hamerle und K.U. Mayer (1986), Ereignisanalyse, Statistische Theorie und Anwendung in den Wirtschafts- und Sozialwissenschaften, Campus Studium, Frankfurt.

Blundell, R., T. Magnac und C.Meghir (1997), Savings and Labor Market Transitions, in: Journal of Business and Economic Statistics, Vol. 15 (2), S. 153-164.

Boockmann, B. und T. Hagen (2001), The Use of Flexible Working Contracts in Germany: Evidence from the Establishment Panel, ZEW Working Paper 01-33.

Bryson, A., R. Dorsett und S. Purdon (2002), The Use of Propensity Score Matching in the Evaluation of Labour Market Policies, Working Paper No. 4, Department of Work and Pension. 
Böhm, P. und Merz, J. (2008), Zum Einkommensreichtum Älterer in Deutschland- Neue Reichtumskennzahlen und Ergebnisse aus der Lohn- und Einkommensteuerstatistik (FAST 2001), FFB-Diskussionspapier Nr. 73, Lüneburg.

Börsch-Supan, A. (1992), Population Aging, Social Security Design and Early Retirement, in: Journal of Institutional and Theoretical Economics, 148, S. 533-557.

Börsch-Supan, A. (1998), Incentive Effects of Social Security on Labor Force Participation: Evidence in Germany and Across Europe, NBER Working Paper no. 6780, Cam-bridge (Mass.).

Börsch-Supan, A. und B. Berkel (2004), Pension Reform in Germany: The Impact on Retirement in Decisions, in: Finanzarchiv, 60(3), S. 393-421.

Börsch-Supan, A. und R. Schnabel (1998a), Social Security and Declining Labor Force Participation in Germany, in: American Economic Review, Bd. 88(2), S. 173- 178.

Börsch-Supan, A. und R. Schnabel (1998b), Social security and retirement in Germany, in J. Gruber \& D.A. Wise: Social security and retirement around the world, S. 135-181, Chicago: The University of Chicago Press.

Börsch-Supan, A., I. Düzgün und M. Weiss (2005), Altern und Produktivität: Zum Stand der Forschung, MEA discussion paper 73-2005.

Boockmann, B. und S. Steffes (2005), Individual and Plant-level Determinants of Job Durations in Germany, ZEW discussion paper 89-05, Mannheim.

Boockmann, B. und T. Zwick (2004), Betriebliche Determinanten der Beschäftigung älterer Arbeitnehmer, in: Zeitschrift für Arbeitsmarktforschung, Jg.35(1), S. 53-63.

Borghans, L. und B. ter Weel (2002), Do Older Workers Have More Trouble Using a Computer than Younger Workers?, in: Research in Labor Economics, 21, S. 139-173.

Boskin, M. J. und M. D. Hurd (1978), The Effect of Social Security on Early Retirement, in: Journal of Public Economics, Bd. 10, S. 361-377.

Bound, J. (1991), Self-reported health vs. objective measures of health in retirement models, in: Journal of Human Resources, Bd. 24, S. 106-138.

Brussig, M., M. Knuth und O. Schweer (2006), Arbeitsmarktpolitik für ältere Arbeitslose Erfahrungen mit „Entgeltsicherung“ und „Beitragsbonus“, IAT-Report 2006-02, Gelsenkirchen.

Büchel, F. und M. Pannenberg (2004), Berufliche Weiterbildung in Ost- und Westdeutschland, in: Zeitschrift für Arbeitsmarktforschung, Bd.37(2), S. 73-125.

Buddelmeyer, H. und E. Skoufias (2003), An Evaluation of the Performance of Regression Discontinuity Design on PROGRESA, IZA discussion paper No.827, Bonn. 
Büttner, R. (2004), Die Wirkungsweise der Rentenreformen auf den Altersübergang, Altersübergangs-Report 2005-01， http://www.iatge.de/auem-report/2005/2005-01/auem200501.pdf, Stand: 4.7.2005.

Büttner, R. und M. Knuth (2004), Spätere Zugänge in Frührenten-Regelaltersrente auf dem Vormarsch, Altersübergangs-Report 2004-01, http://www.iatge.de/auem-report/2004/200401/auem2004-01.pdf, Stand: 4.7.2005.

Büttner, R., Knuth, M. und S. Wojtowski (2005), Die Kluft zwischen Erwerbsaustritt und Renteneintritt wird wieder größer, Altersübergangs-Report 2005-03, http://www.iatge.de/auem-report/2005/2005-03/auem2005-03.pdf, Stand: 4.7.2005.

Bundesagentur für Arbeit (2004), Merkblatt 14: Gleitender Übergang in den Ruhestand, Hinweise für Arbeitgeber und Arbeitnehmer, Nürnberg.

Bundesministerium für Gesundheit und Soziale Sicherung (2005), Ratgeber zur Rente, Berlin.

Burgert, D. (2006a), Einstellungschancen von Älteren - Wie wirkt der Schwellenwert im Kündigungsschutz?, FFB-Diskussionspapier Nr. 62, Lüneburg.

Burgert, D. (2006b), The Impact of German Job Protection Legislation on Job Creation in Small Establishments, in: Applied Economics Quarterly, Bd. 52/2, S. 123-140.

Burgert, D. (2006c), Betriebliche Weiterbildung und Verbleib Älterer im Betrieb, FFBDiskussionspapier Nr. 68, Lüneburg.

Burgert, D. (2007), Betriebliche Weiterbildung und Verbleib Älterer im Betrieb, RatSWD Research Note No. 2.

Burgert, D. (2008), Einstellungschancen von Älteren - Wie wirkt der Schwellenwert im Kündigungsschutz?, in: J. Merz: „Freie Berufe, Einkommen und Steuern, im Erscheinen, Lüneburg.

Caliendo, M. und S. Kopeinig (2005), Some Practical Guidance for the Implementation of Propensity Score Matching, in: Journal of Economic Surveys, IZA discussion paper No. 1588, Bonn. (Erscheint in: Journal of Economic Surveys)

Carling, K. und L. Larsson (2002), Does early intervention help the unemployed youth?, IFAU Working Paper 2002:10, Uppsala.

Cameron, A. und P. Trivedi (2005), Microeconometrics - Methods and Applications, Cambridge University Press, Cambridge.

Chan, S. und A. H. Stevens (1999), Employment and Retirement following a Late-Career Job Loss, in: American Economic Review, 89, S. 211-216.

Chennells, L. und J. Van Reenen (2002), Has Technology Hurt Less Skilled Workers? An Econometric Study of the Effects of Technical Change on the Structure of Pay and Jobs, in: 
L'Horty, Y., N. Greenan and J. Mairesse Hrsg.: Productivity, Inequality and the Digital Economy, MIT Press: Boston, S. 175-225.

Christensen, B. (2001), Berufliche Weiterbildung und Arbeitsplatzrisiko: Ein MatchingAnsatz, Kieler Arbeitspapier Nr. 1033, Kiel.

Christensen, B. (2003), Anspruchslohn und Arbeitslosigkeit in Deutschland, in: Mitteilungen aus der Arbeitsmarkt- und Berufsforschung, Bd. 31, S. 205-225.

Christensen, B.J. und N. Datta Gupta (1994), A dynamic programming model of the retirement behaviour of married couples, CAE Working Paper 94.

Clewes, M, W. Gould und R. Gutierrez (2004), An Introduction to Survival Analysis Using Stata, Revised Version, StataPress, College Station, Texas.

Coile, C. (2003), Retirement Incentives and Couples' Retirement Decisions, NBER Working Paper No. 9496.

Coile, C. und P. Levine (2004), Bulls, bears and Retirement Behavior, NBER Working Paper No. 10779.

Deary, I., L. Whalley, H. Lemmon, J. Crawford und J. Starr (2000), The stability of individual differences in mental ability from childhood to old age: follow-up of the 1932 Scottish Mental Survey, in: Intelligence, Bd. 28, S. 49-55.

Deutscher Bundestag (2006), Die Wirksamkeit moderner Dienstleistungen am Arbeitsmarkt, Bundestagsdrucksache 16/505, Berlin.

DiNardo J. und J. S. Pischke (1997), The return to computer use revisited: have pencils changed the wage structure too?, in: Quarterly Journal of Economics, Bd. 112, S. 291-303.

DRV (2008), Rentenversicherung in Zeitreihen, Frankfurt.

Düll, H. und L. Bellmann (1998), Betriebliche Weiterbildungsaktivitäten in West- und Ostdeutschland - eine theoretische und empirische Analyse mit den Daten des IABBetriebspanels 1997, in: Mitteilungen aus der Arbeitsmarkt- und Berufsforschung, Bd. 31, S. 205-225.

Dwyer, D. (2001), Planning for Retirement: The Accuracy of Expected Retirement Dates and the Role of Health Shocks, CRR working paper 2001-48.

Dwyer, D. und O. Mitchell (1998), Health Problems as Determinants for Retirement: Are Self-Rated Measures Endogenous?, in: Journal of Health Economics, Bd. 18, S. 173-193.

Eichhorst, W (2006), Beschäftigung Älterer in Deutschland: Der unvollständige Paradigmenwechsel, IZA discussion paper No. 1985, Bonn.

Eichhorst, W. und C. Sproß (2005), IAB-Kurzbericht No.16, Nürnberg. 
Engstler, H. (2004), Geplantes und realisiertes Austritts alter aus dem Erwerbsleben, DZADiskussionspapier No. 41, Berlin.

Estevez-Abe, M., T. Iversen und D. Soskice (2001), Social Protection and the Formation of Skills: A Reinterpretation of the Welfare State, in: Peter A. Hall und David Soskice (Hg.): Varieties of Capitalism. The Institutional Foundations of Comparative Advantage, S. 145183, Oxford University Press, Oxford.

Europäische Kommission (2003), Indicators for Monitoring - the 2003 Employment Guidelines, Brüssel.

Fan, J. und I. Gijbels (1996), Local Polynomial Modelling and Its Applications, London.

Feldstein, M. (1974), Social security, induced retirement and aggregate capital accumulation, in: Journal of Political Economy, Bd. 82, S. 902-926.

Fitzenberger, B., H. Prey (1999), Berufliche Weiterbildung und die Stabilität der Beschäftigung - eine Evaluation auf Basis des Sozio-Ökonomischen Panels, in: D. Beer et al. (Hrsg.): Die wirtschaftlichen Folgen von Aus- und Weiterbildung, München.

Fitzenberger, B., A. Garloff und K. Kohn (2003), Beschäftigung und Lohnstrukturen nach Qualifikation und Altersgruppen: Eine empirische Analyse auf Basis der IAB-Beschäftigtenstichprobe, ZEW Discussion Paper 03-75.

Fitzenberger, B., A. Osikominu und R. Wolter (2005), Imputation Rules to Improve the Education Variable in the IAB Employment Subsample, FDZ Methodenreport 03/2005, Nürnberg.

Fitzenberger, B. und R. Wilke (2004), Unemployment Durations in West-Germany Before and After the Reform of the Unemployment Compensation System During the 1980s, ZEW Discussion Paper No. 04-24, Mannheim.

Friedberg, L. (2003), The Impact of Technological Change on Older Workers: Evidence from Data on Computer Use, in: Industrial and Labor Relations Review, 56 (3), S. 511- 529.

Friedrich, W und H. Hägele (1997), Ökonomische Konsequenzen vom Schwellenwerten im Arbeits- und Sozialrecht sowie die Auswirkungen dieser Regelungen, Studien der ISG Sozialforschung und Gesellschaftspolitik, 20, Köln.

Frosch, K. (2007), Einfluss soziodemographischer Faktoren und der Erwerbsbiographie auf die Reintegration von Arbeitssuchenden: Schlechte Chancen ab Alter 50?, Rostocker Diskussionspapier Nr. 11, Rostock.

Funk, L. (2004), Employment Opportunities for Older Workers: A Comparison of Selected OECD Countries, in: CESifo DICE Report, S. 22-33.

Garibaldi, P.; Pacelli L. und A. Borgarello (2003), Employment Protection Legislation and the Size of Firms, IZA discussion paper No. 787, Bonn. 
Gartner, H. (2005), The Imputation of Wages above the Contribution Limit with the German IAB Employment Sample, FDZ Methodenreport Nr.2/2005, Nürnberg.

Gatter, J. (2004), Personalpolitik und alternde Gesellschaft: betriebliche Ursachen für die Persistenz der Frühverrentung aus Sicht der Neuen Institutionenökonomie, Hampp, München.

Gatter, J. und B. K. Hartmann (1995), Betriebliche Verrentungspraktiken zwischen arbeitsmarkt- und rentenpolitischen Interessen, Sonderdruck aus: Mitteilungen aus der Arbeitsmarkt- und Berufsforschung.

Gerfin, M. (2004), Work-Related Training and Wages: An Empirical Analysis for Male Workers in Switzerland, IZA discussion paper No.1078, Bonn.

Gerlach K. und U. Jirjahn (1998), Determinanten betrieblicher Weiterbildungsaktivitäten: Eine empirische Untersuchung mit Daten des Hannoveraner Firmenpanels, in: F. Pfeiffer und W. Pohlmeier (Hrsg.): Qualifikation, Weiterbildung und Arbeitsmarkterfolg, Baden-Baden, Nomos, S. 311-337.

Gustman, A., und T. Steinmeier (1986), A structural retirement model, in: Econometrica, Vol. 54, S. 555-584.

Gustman, A., und T. Steinmeier (1994), Retirement in a Family Context: A Structural Model for Husbands and Wives, NBER Working Paper No. 4629.

Haan P. und V. Steiner (2006), Making Work Pay for the Elderly Unemployed -Evaluating Alternative Policy Reforms for Germany, DIW Discussion Paper No. 641, Berlin.

Hahn, J.; Todd, P. und W. Van der Klaauw (1999), Evaluating the Effect of an Antidiscrimination Law Using a Regression Discontinuity Design, NBER Working Paper 7131.

Hahn, J.; Todd, P. und W. Van der Klaauw (2001), Identification and Estimation of Treatment Effects with a Regression Discontinuity Design, in: Econometrica, 69 (1), 201-209.

Hamermesh, D.S. (1993), Labor Demand, Princeton University Press, Princeton.

Hægeland, T., D. Rønningen and K. G. Salvanes: Adapt or withdraw? Evidence on technological changes and early retirement using matched worker-firm data, Statistics Norway, Discussion Papers No. 509, Oslo.

Heckman, J.J., LaLonde, R.J. und J. Smith, (1999), The Economics and Econometrics of Active Labor Market Programs, in: Ashenfelter, O. und D. Card. (Hrsg.): Handbook of Labor Economics, Vol. IIIA, S. 1865-2097.

Helberger, C. und J. Schwarze (1986), Umfang und Struktur der Nebenerwerbstätigkeit in der Bundesrepublik Deutschland, in: Mitteilungen für Arbeitsmarkt- und Berufsforschung, Bd. 2, S. 271-286.

Heyma, A. (2001), Dynamic models of labour force retirement: an empirical analysis of Early Exit in the Netherlands, Tinbergen Institute Research Series; 257. 
Heyma, A. (2004), A Structural Dynamic Analysis of Retirement Behaviour in the Netherlands, in: Journal of Applied Econometrics, S. 739-759.

Heywood, J., Lok-Sang Ho und X. Wei (1999), The Determinants of Hiring Older Workers: Evidence from Hong-Kong, in: Industrial and Labor Relations Review, Bd. 52(3), S. 444-459.

Hirsch, B. T., MacPherson D.A. und M.-C. Hardy (2000), Occupational Age Structure and Access for Older Workers, in: Industrial and Labor Relations Review, Bd. 53(3), S. 401-418.

Hoffmann, E. (1996), Frühverrentung und Beschäftigung - Ältere Arbeitnehmer zwischen Beschäftigung, Arbeitslosigkeit und Ruhestand 1980-1995, IAB-Kurzbericht Nr. 9, Nürnberg.

Horn, J. L. und R. B. Cattell (1966), Refinement and Test of the Theory of Fluid and Crystallized Intelligence, in: Journal of Educational Psychology, Bd. 57, S. 253-270.

Horn, J. L. und R. B. Cattell (1967), Age Differences in Fluid and Crystallized Intelligence, in: Acta Psychologica, Bd. 26, S. 107-129.

Hübler, O. (1998), Berufliche Weiterbildung und Umschulung in Ostdeutschland - Erfahrungen und Perspektiven, in: F. Pfeiffer und W. Pohlmeier (Hrsg.): Qualifikation, Weiterbildung und Arbeitsmarkterfolg. Baden-Baden.

Hujer, R. und D. Radic (2006), Age and Skill Biased Technological Change: A Multiple Treatment Approach Using Linked Employer Employee Dataset, mimeo.

Hurd, M.D. (1990), The Joint Retirement Decisions of Husbands and Wives, in: D.A. Wise (Hrsg.): Issues in the Economics of Aging, University of Chicago Press for the Na-tional Bureau of Economic Research.

Hurd, M. und K. McGarry (1993), The Relationship between Job Characteristics and Retirement, Working Paper No. 4558, Cambridge (Mass.).

Hutchens, R. (1986), Delayed Payment Contracts and a Firms Propensity to Hire Older Workers, in: Journal of Labor Economics, Band 4, S. 439-457.

Hutchens, R. (1987), A Test of Lazear's Theory of Delayed Payment Contracts, in: Journal of Labor Economics, Band 5, S. 153-170.

Ichino, A. und R. Riphahn (2005), The Effect of Employment Protection on Worker Effort. A Comparison of Absenteeism During and After Probation, in: Journal of the European Economic Association, 3(1), S. 120-143.

Jaenichen, U., Kruppe, T., Stephan, G., Ullrich, B. und F. Wießner (2005), You can Split it if you really want, Korrekturvorschläge für ausgewählte Inkonsistenzen in IEB und MTG, FDZDatenreport 4/2005, Nürnberg.

Jahn, E. und C. Schnabel (2003), Bestandsschutz durch Abfindungen: Höhere Rechtssicherheit und Effizienz, in: Wirtschaftsdienst, Bd. 4, S. 219-223.

Jenkins, S. (2005), Panel Data Methods, Part II: Survival Analysis, manuscript, Essex. 
Kirner, E. und V. Meinhardt (1997), Gesetzliche Rentenversicherung: Senkung des Rentenniveaus nicht der richtige Weg, DIW Wochenbericht 24-25/97, Berlin.

Knaus, T. (2003), The Retirement Decision in Germany - a -A Dynamic Programming Approach, Shaker.

Koller, B. (2001), Das Rentenalter wurde angehoben - zieht der Arbeitsmarkt mit?, IAB Werkstattbericht Nr.7, Nürnberg.

Koller, L. (2005), Arbeitsrechtliche Schwellenwerte - Regelungen an der Schwelle zur Unüberschaubarkeit, Discussion paper No. 40, Lehrstuhl für Arbeitsmarkt- und Regionalpolitik, Nürnberg.

Kölling, A. (2000):, The IAB-Establishment Panel, Schmollers Jahrbuch, Zeitschrift für Wirtschafts- und Sozialwissenschaften 120(2), S. 291-300.

Kruger, A. B. (1993), How computers have changed the wage structure: evidence from microdata, 1984-1989, in: Quarterly Journal of Economics, Bd. 108, S. 33-60.

Lancaster, T. (1990), The Econometric Analysis of Transition Data. Cambridge University Press.

Larsen, M. und N. Datta Gupta (2004), The Impact of Health on Individual Retirement Plans: a Panel Analysis comparing Self-reported versus Diagnostic Measures, Working paper 04-7, Department of Economics, Aarhus School of Business.

Lazear, E. (1979), Why is there mandatory retirement?, in: Journal of Political Economy, 87, S. 1261-1284.

Lazear, E. (1990), Job Security Provisions and Employment, in: Quarterly Journal of Economics, Vol. 105, S. 699-726.

Lechner, M. (2001), A note on the common support problem in applied evaluation studies, Discussion Paper 2001-01, Department of Economics, University of St.Gallen.

Leigh, D. (1984), Why is there Mandatory Retirement? An Empirical Reexamination, in: Journal of Human Resources 19 (4): 512-531.

Leuven, E. (2005), The Economics of Private Sector Training: A Survey of the Literature, in: Journal of Economic Surveys, Bd. 19(1), S. 91-111.

Leuven, E. und H. Oosterbeek (2004), Evaluating the effect of tax deductions on training, in: Journal of Labor Economics Bd. 22(1), S. 461-488.

Leuven, E. und B. Sianesi (2003), PSMATCH2: Stata Module to Perform Full Mahalanobis and Propensity Score Matching, Common Support Graphing and Covariate Imbalance Testing, http://ideas.repec.org/c/boc/bocode/s432001.html.

Levy, F. und R.J. Murnane (1996), With what skills are computers a complement?, in: American Economic Review, Bd. 86, S. 258-262. 
Lindeboom, M. und M. Kerkhofs (2002), Health and Work of the Elderly - Subjective Health Measures, Reporting Errors and the Endogenous relationship between Health and Work, IZA-Discussion Paper No. 457, Bonn.

Loewenstein G. und N. Sicherman (1991), Do Workers Prefer Increasing Wage Profiles?, in: Journal of Labor Economics, Vol. 9, No.1, S. 67-84.

Lumsdaine, R. und O.S. Mitchell (1999), New Developments in the Analysis of Retirement Behavior, in: O.Ashenfelter und D.Card: Handbook of Labour Economics, Vol. 3C.

Mastrogiacomo, M., R. Alessie und M. Lindeboom (2004), Retirement Behaviour of Dutch Elderly Households, in: Journal of Applied Econometrics, Bd. 19, S. 777-793.

McGarry, K. (2002), Health and Retirement: Do Changes in Health Affect Retirement Expectations?, NBER Working Paper No.9317, Cambridge (Mass.).

Merz, J., C. Schatz und K. Kortmann (2004), Mikrosimulation mit Verwaltungs- und Befragungsdaten am Beispiel ,Altersvorsorge in Deutschland 1996' (AVID ,96), in: Merz, J. und M. Zwick (Hg.), MIKAS - Mikroanalysen und amtliche Statistik, Statistik und Wissenschaft, Vol. 1, S. 231-248.

Michaelis, K. und C. Rieckhoff (2003), Anreize im Rentenrecht zur Erwerbsbeteiligung Älterer, in: Mitteilungen der deutschen Angestelltenversicherung, 01/03, S. 6-11, Berlin.

Mincer, J. (1974), Schooling, Experience, and Earnings. NBER, Cambridge (Mass.).

Mortensen, D. T. (1978), Specific capital and labor turnover, in: Bell Journal of Economics, Band 9 (2), S. 572-586.

OECD (1999), Employment Outlook 1999, Paris.

OECD (2004), OECD Employment Outlook 2004, Paris.

OECD (2005), Ageing and Employment Policies: Germany. Paris.

Oster, S. und D. Hamermesh (1998), Aging and Productivity among Economists, in: The Review of Economics and Statistics, Bd. 80 (1), S. 154-156.

Parent, D. (1999), Wages and Mobility: The Impact of Employer-Provided Training, in: Journal of Labor Economics, Bd.17 (2), S. 298-317.

Park, D., R. Nisbett und T. Hedden (1999), Culture, Cognition and Aging, in: Journal of Gerontology, Bd. 54 B, S. 75-84.

Pischner, R. und G. Wagner (1992), Zwei Aspekte der Flexibilität beim Übergang vom Erwerbsleben in den Ruhestand, in: Hujer, R., Schneider, H. und W. Zapf (Hrg.): Herausforderungen an den Wohlfahrtsstaat im strukturellen Wandel, S. 99-115, Campus, Frankfurt.

Porter, J. (2003), Estimation in the Regression Discontinuity Model, unpublished manuscript. 
Quinn, J. (1977), Microeconomic determinants of early retirement: a cross-sectional View of white married men, in: Journal of Human Resource, Bd.12, S. 329-347.

Quinn, J., R. Birkhauser und D. Myers (1990), Passing the Torch: The Influence of Economic Incentives on Work and Retirement, Upjohn Institute, Kalamazoo.

Riach, P.A. und J. Rich (2006), An Experimental Investigation of Age Discrimination in the French Labour Market, IZA discussion paper no. 2522, Bonn.

Riphahn, R. und P.Schmidt (1997), Determinanten des Rentenzugangs: Eine Analyse altersspezifischer Verrentungsraten, in: Jahrbücher für Wirtschaftswissenschaften, Bd. 48, S. 133147.

Riphahn, R. (2004), Employment Protection and Effort Among German Employees, in: Economics Letters, 85(3), S. 353-357.

Riphahn R. und A. Thalmaier (2001), Behavioral Effects of Probation Periods: An Analysis of Worker Absenteeism, in: Journal of Economics and Statistics, 221(2), S. 179-201.

Ridder, G. und I. Tunali (1999), Stratified partial likelihood estimation, in: Journal of Econometrics 92(2), S. 193-232.

Rosenbaum, P.R. und D.B. Rubin (1983), The Central Role of the Propensity Score in Observational Studies for Causal Effects, in: Biometrika, Bd. 70, S. 41-55.

Rosenow, J. und G. Wagner (1994), Das staatliche Rentenzugangsinstrumentarium und die Entwicklung des Rentenzugangs, in: Rosenow, J. und F. Naschold: Die Regulierung von Altersgrenzen, edition sigma, Berlin.

Roy, A. (1951), Some Thoughts on the Distribution of Earnings, in: Oxford Economic Papers, Bd. 3, S. 135-146.

Rubin D. B. (1974), Estimating Causal Effects of Treatments in Randomized and Nonrandomized Studies, in: Journal of Educational Psychology, Bd. 66, S. 688-701.

Rubin D. B. und N. Thomas (1996), Matching Using Estimated Propensity Scores: Relating Theory to Practice, in: Biometrics, Bd. 52, S. 249-264.

Rust, J. (1989), A dynamic programming model of retirement behaviour, in. D. A, Wise (Hrsg.) The economics of aging, S. 359-403, Chicago: The University of Chicago Press.

Rust, J., Phelan, C. (1997), How social security and Medicare affect Retirement behaviour in a world of incomplete markets, in: Econometrica, Bd. 86, S. 781-831.

Sammartino, F. (1987), The effect of health on retirement, in: Social security Bulletin, Bd. 50 (2), S. 31-47.

Schasse, U. (1991), Betriebszugehörigkeitsdauer und Mobilität - Eine empirische Untersuchung zur Stabilität von Beschäftigungsverhältnissen, Campus, Frankfurt. 
Schatz, K. und J. Merz (2000), Die Rentenreform in der Diskussion -Ein Mikrosimulationsmodell für die Altersvorsorge in Deutschland (AVID-PRO), FFB-Diskussionspapier Nr. 28, Lüneburg.

Schleife, K. (2006), Computer Use and the Employment Status of Older Workers - An Analysis based on Individual Data, in: LABOUR: Review of Labour Economics and Industrial Relations 20 (2), S. 325-348.

Schleife, K. (2008), IT Training and the Employability of Older Workers, ZEW Discussion Paper 08-021.

Schmidt, M. (2003), The need for Modernising German Labour Law arising from the ban of age discrimination, in: Blaupin, R. und M.Weiss (Hrg.): Changing Industrial Relations and Modernisation of Labour Law, Kluwer.

Schmidt, P. (1995), Die Wahl des Rentenalters - Theoretische und empirische Analyse des Rentenzugangsverhaltens in West- und Ostdeutschland, Verlag Peter Lang, Frankfurt.

Schøne, P. (2009), New technologies, new work practices and the age structure of the workers, in: Journal of Population Economics 22 (3), S. 803-826.

Siddiqui, S. (1997), The pension incentive to retire: empirical evidence for West-Germany, in: Journal of Population Economics, 10, S. 463-486.

Skirbekk, V. (2003), Age and Individual Productivity: A Literature Survey, MPIDR Working paper 2003-028.

Statistisches Bundesamt (2007), Berufliche Weiterbildung in Unternehmen - Dritte europäische Erhebung über die berufliche Weiterbildung in Unternehmen, Wiesbaden.

Strotmann, H. und W. Hess (2003), Eigenschaften und Beschäftigungschancen älterer Arbeitnehmer sowie betriebliche Maßnahmen für ältere Arbeitnehmer in Baden-Württemberg, IAW Short report 7, Tübingen.

Stück, Heiner (2003), Gleitend in den Ruhestand? Gesetzliche, tarifliche und tastsächliche Entwicklung der Altersteilzeit, Arbeitnehmerkammer Bremen (Hg.), Bremen.

Thon, M. (1996), Frühverrentung und Beschäftigung - Demographische Entwicklung und rechtliche Grundlagen, IAB-Kurzbericht Nr.8, Nürnberg.

VDR (1997), .Das Rentenreformgesetz 1999, Frankfurt.

VDR (2001), Rentenreform 2001 - Das Wichtigste im Überblick, Frankfurt.

Verick, S. (2004), Threshold Effects of Dismissal Protection Legislation in Germany, IZA discussion paper No. 991, Bonn.

Viehbrok, H. (1997), Das Arbeitsangebot im Übergang von der Beschäftigung in den Ruhestand, eine Analyse der Arbeitsanreize durch sozialrechtliche Regelungen in der Spätphase des Erwerbslebens, Frankfurt a.M. 
Viehbrok, H. (1998), Der Übergang in den Ruhestand als mehrstufiger Entscheidungsprozeß, in: W.R. Heinz, D. Blaschke und G. Engelbrech: Was prägt Berufbiographien? Lebenslaufdynamik und Institutionenpolitik, Beiträge zur Arbeitsmarkt- und Berufsforschung, Bd. 15, Nürnberg.

Wagner, J.; Schnabel C. und A. Kölling (2001a), Threshold Values in German Labor Law and Job Dynamics in Small Firms: The Case of the Disability Law, in: IFO Studien, 47, S. 65 $-75$.

Wagner, J.; Schnabel C. und A. Kölling (2001b), Wirken Schwellenwerte im deutschen Arbeitsrecht als Bremse für die Arbeitsplatzbeschaffung in Kleinbetrieben?; in: Ehrig D. und P. Kalmbach (eds.): Weniger Arbeitslose - aber wie?, Marburg, S. 177-198.

Weinberg, B.A. (2001), Experience and technology adaption, mimeo, Ohio State University.

Wilke, R. A. (2004), New Estimates of the Duration and Risk of Unemployment for WestGermany, in: Journal of Applied Social Science Studies; Bd. 125(2) S. 207-237.

Winkelmann, R. und K. Zimmermann (1998), Is job stability declining in Germany? Evidence from count data models, in: Applied Economics, Bd. 30, S. 1413-1420.

Wübbeke, C. (1999), Der Übergang von sozialversicherungspflichtiger Beschäftigung in den Rentenbezug zwischen sozialpolitischer Steuerung und betrieblichen Interessen, in: Mitteilungen aus der Arbeitsmarkt- und Berufsforschung, Bd. 32, S. 102-117.

Wübbeke, C. (2005a), Der Übergang in den Rentenbezug im Spannungsfeld betrieblicher Personal- und staatlicher Sozialpolitik (Textband), Beiträge zur Arbeitsmarkt- und Berufsforschung 290.2, Nürnberg.

Wübbeke, C. (2005b), Der Übergang in den Rentenbezug im Spannungsfeld betrieblicher Personal- und staatlicher Sozialpolitik (Anhangband), Beiträge zur Arbeitsmarkt- und Berufsforschung 290.2, Nürnberg.

ZEW, IAB, IAT (2005), Evaluation der Maßnahmen zur Umsetzung der Vorschläge der Hartz-Kommission, Arbeitspaket 1: Wirksamkeit der Instrumente -Modul 1d: Eingliederungszuschüsse und Entgeltsicherung, Bericht 2005, Mannheim, Nürnberg, Gelsenkirchen.

Zhao, Z. (2004), Using Matching to Estimate Treatment Effects: Data Requirements, Matching Metrics and Monte Carlo Evidence, in: The Review of Economics and Statistics, Bd. 86(1), S. 91-107.

Zöllner, D. (1997), Soziale Sicherung, Oldenbourg Verlag, München. 